University of Louisville

ThinkIR: The University of Louisville's Institutional Repository

$5-2011$

\title{
Riparian corridor vegetation structure and soil function along urban, suburban, and rural streams in Louisville, KY, USA.
}

R. Jonathan White

University of Louisville

Follow this and additional works at: https://ir.library.louisville.edu/etd

\section{Recommended Citation}

White, R. Jonathan, "Riparian corridor vegetation structure and soil function along urban, suburban, and rural streams in Louisville, KY, USA." (2011). Electronic Theses and Dissertations. Paper 1555.

https://doi.org/10.18297/etd/1555

This Doctoral Dissertation is brought to you for free and open access by ThinkIR: The University of Louisville's Institutional Repository. It has been accepted for inclusion in Electronic Theses and Dissertations by an authorized administrator of ThinkIR: The University of Louisville's Institutional Repository. This title appears here courtesy of the author, who has retained all other copyrights. For more information, please contact thinkir@louisville.edu. 


\title{
RIPARIAN CORRIDOR VEGETATION STRUCTURE AND SOIL FUNCTION ALONG URBAN, SUBURBAN, AND RURAL STREAMS IN LOUISVILLE, KY, USA
}

By

R. Jonathan White

B. S., Western Carolina University, 1996

M. S., Western Carolina University, 2001

\author{
A Dissertation \\ Submitted to the Faculty of the \\ College of Arts and Sciences of the University of Louisville \\ in Partial Fulfillment of the Requirements \\ for the Degree of
}

Doctor of Philosophy

Department of Biology

University of Louisville

Louisville, KY

May 2011 

RIPARIAN CORRIDOR VEGETATION STRUCTURE AND SOIL FUNCTION ALONG URBAN, SUBURBAN, AND RURAL STREAMS IN LOUISVILLE, KY, USA

By

R. Jonathan White

B.S., Western Carolina University, 1996

M.S., Western Carolina University, 2001

A Dissertation Approved on

November 15, 2010

by the following Dissertation Committee:

Dissertation Director (Margaret M. Carreiro)

James E. Alexander

Sarah Emery

Hwa-Seong Jin

Wayne Zipperer 


\section{ACKNOWLEDGEMENTS}

I would like to thank my committee chair, Dr. Margaret Carreiro, as well as my current committee members Dr. Wayne Zipperer, Dr. James Alexander, Dr. Sarah Emery, and Dr. Hwa-Seong Jin. My committee, under the guidance of Dr. Carreiro, has contributed greatly to the quality of this dissertation as well as my scientific endeavors. I would also like to thank former and current lab members for field (Alice Hoang, Brad Didier, and Meghan Langley) and technical assistance (Tara Trammell and Lydia Waters). In collaboration with the University of Louisville, the USDA Forest Service, SRS-4952 and Metropolitan Sewer District (MSD) of Louisville provided funds to conduct field analyses on riparian habitats. Funds were also provided by the Kentucky Society of Natural History. This dissertation would not have been possible without the assistance of Pat Haragan, who identified many of the plants I collected, and Jacqueline White, who provided a great deal of statistical and methodological support.

I wish to acknowledge my Aunt Susan and Uncle Ray for encouraging me to pursue doctoral studies. My deepest gratitude goes to my mother and family for their continual support no matter the paths I chose. Finally, I can only express in joy and tears the contributions and sacrifices of my wife, Xin (我爱你). 


\begin{abstract}
RIPARIAN CORRIDOR VEGETATION STRUCTURE AND SOIL FUNCTION ALONG URBAN, SUBURBAN, AND RURAL STREAMS IN LOUISVILLE, KY, USA
\end{abstract}

\author{
R. Jonathan White
}

May 13, 2011

Stream riparian zones are ecotones between terrestrial and aquatic environments.

Studying these areas in urban environments is important since they lie adjacent to stream water supplies. I conducted a study of riparian woody and groundcover vegetation along urban, suburban, and rural streams (land-use designations based on \% impervious surface) to assess how cities are affecting plant community structure. I also studied riparian soil gas flux (carbon dioxide, methane, and nitrous oxide) in relation to water table depth and groundwater nutrient concentrations spanning a 10-month period (January to October, 2008). I found distinct woody and groundcover communities associated with proportion of impervious surface surrounding the research sites. These communities differed regarding diversity and proportion of native, exotic, and wetland species distributions. The most urban communities generally had lower species richness, more exotic species, and fewer wetland species when compared to rural areas. Urban areas also exhibited the highest streambanks and lowest water tables. Carbon dioxide gas flux rates were higher in urban areas, but methane and nitrous oxide fluxes did not respond uniformly to site differences as classified by proportion of impervious surface. 
Methane and nitrous oxide differences, in addition to seasonal variability, were more greatly affected by local site level differences in substrate and nutrient ratios as well as soil moisture. 


\section{TABLE OF CONTENTS}

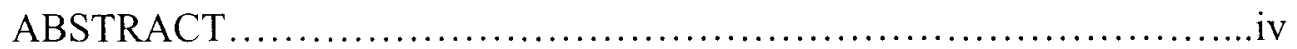

LIST OF TABLES ........................................................

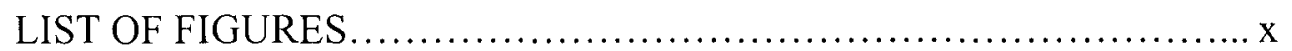

Chapter 1: Introduction................................................ 1

Chapter 2: Variation in woody streambank plant communities along urban, suburban, and rural streams in metro Louisville, Kentucky............. 14

Introduction................................................... 14

Methods......................................................... 17

Results....................................................... 33

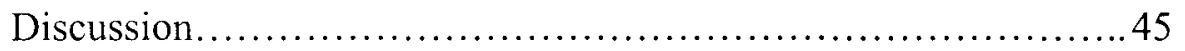

Conclusions................................................. 51

Chapter 3: Variation in riparian groundcover plant communities surrounded by urban, suburban, and rural land-use in Louisville,

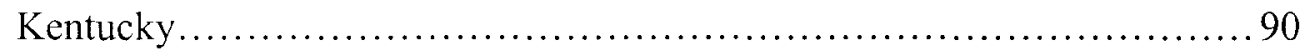

Introduction................................................... 90

Methods..................................................... 94

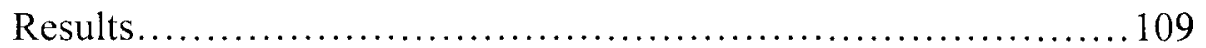

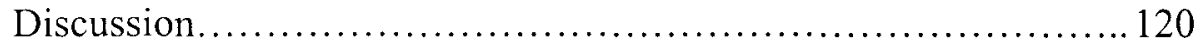

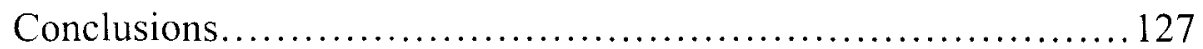

Chapter 4: Seasonal greenhouse gas flux in riparian soils along urban, suburban, and rural riparian zones in metro Louisville, Kentucky .............. 155

Introduction.................................................... 155

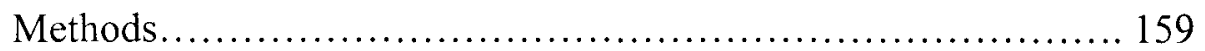

Results..................................................... 172

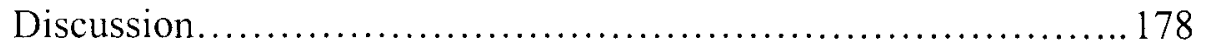

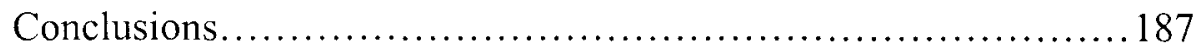

Chapter 5: Conclusions.................................................. 215

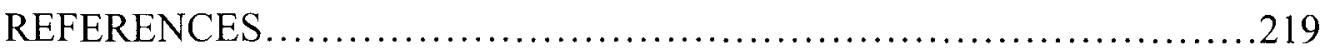

APPENDICES

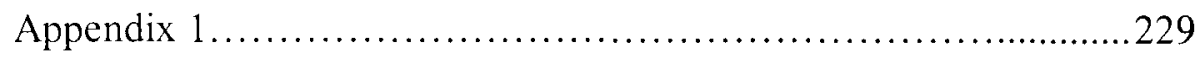

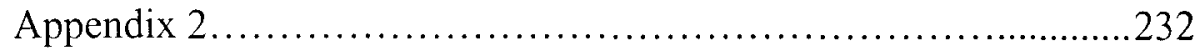




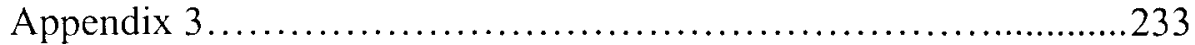

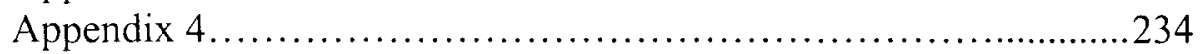

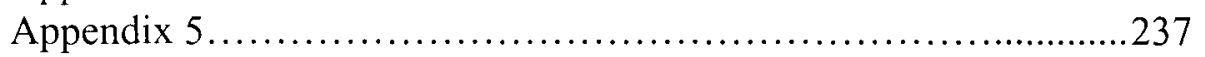

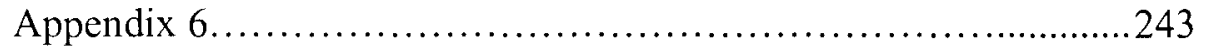

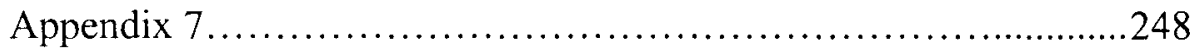

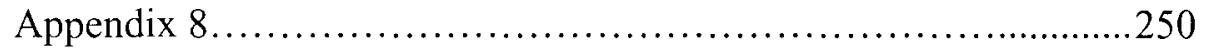

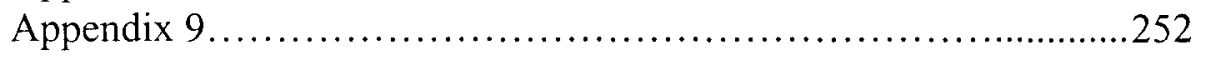

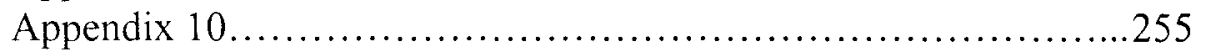

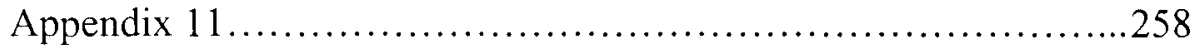

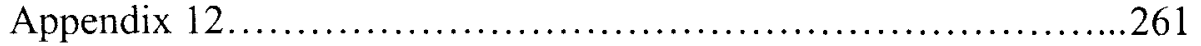

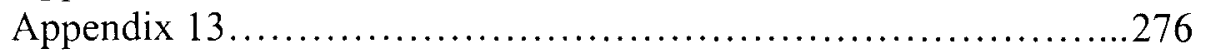

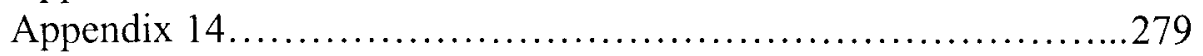

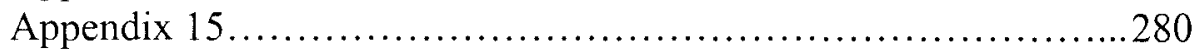

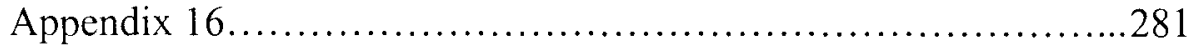

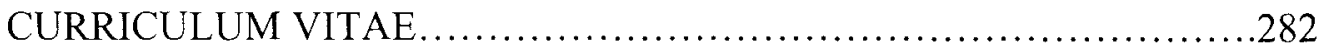




\section{LIST OF TABLES}

Table 2-1: Area of research catchments and the number of study sites classified as urban, suburban and rural at the subcatchment scale, and within a $1-\mathrm{km}$ and 500-m radius from each study site.

Table 2-2: Distribution of riparian width intervals by stream order and land-use categories at the subcatchment scale.

Table 2-3: Relative importance value of dominant ( $>5 \%$ ) woody species by strata within land-use classes.

Table 2-4: Tree frequency, mean density and basal area ( \pm S.E.) in landuse categories at the subcatchment scale...

Table 2-5: Sapling frequency and mean density (+ S.E.) in land-use categories at the subcatchment scale.

Table 2-6: Tree seedling frequency and mean density (+ S.E.) in land-use categories at the subcatchment scale...

Table 2-7: Shrub frequency and mean density (+ S.E.) in land-use categories at the subcatchment scale

Table 2-8: Tree diameter distribution in riparian zones by land-use category at the subcatchment scale.

Table 2-9: ANOVA of woody plant richness by plant type among land use categories at the subcatchment scale.

Table 2-10: Tukeys HSD pairwise comparisons of tree seedling richness by land-use category at the subcatchment scale....

Table 2-11: Woody plant species composition for the most common species and significant indicator values for a 3-cluster configuration using optimal partitioning...

Table 2-12: Woody plant species composition and significant indicator values for 5-cluster configuration using optimal partitioning. 
Table 2-13: Environmental and National Land Cover Database (NLCD) metrics used for non-metric multidimensional scaling.

Table 3-1: Area of research catchments and the number of study sites classified as urban, suburban and rural at the subcatchment scale, and within a $1-\mathrm{km}$ and $500-\mathrm{m}$ radius from each study site

Table 3-2: Distribution of riparian width intervals in this study by stream order and land use categories at the subcatchment scale.

Table 3-3: Mean \% cover (+S.E.) for the top ten most abundant herb and woody vine species in urban, suburban and rural land-use categories....131

Table 3-4: Riparian groundcover mean species richness by distance from the stream bank and land use categories determined at the subcatchment scale.

Table 3-5: Frequently occurring and significant indicator species (using indicator species analysis) for 3-cluster configuration using complete clustering.

Table 3-6: Environmental and National Land Cover Database (NLCD) metrics used for non-metric multidimensional scaling.

Table 4-1: Gravimetric soil water content (g water/g oven dry soil) in the upper $15-\mathrm{cm}$ horizons within two meters of streambanks in urban, suburban, and rural riparian sites.

Table 4-2: Site mean vegetation data surrounding the static chambers at riparian sites

Table 4-3: Riparian groundwater nitrate+nitrite-N monthly means $( \pm$ S.E.) for subcatchment land-use categories and total monthly rainfall from November 2007 to October 2008.

Table 4-4: Riparian groundwater dissolved organic carbon (DOC) monthly means $( \pm$ S.E.) for subcatchment land-use categories and total monthly rainfall

Table 4-5: Relationships between landscape-level soil factors and $\mathrm{CO}_{2}$ and $\mathrm{CH}_{4}$ fluxes in riparian areas across an urban-to-rural land-use gradient. 194

Table 4-6: Relationships between plot-level soil factors and $\mathrm{CO}_{2}$ and $\mathrm{CH}_{4}$ fluxes in riparian areas across an urban-to-rural land-use gradient 


\section{LIST OF FIGURES}

Figure 1-1: Conceptual model of variables that affect and are affected by vegetation within a riparian area.

Figure 2-1: Schematic map of the three watersheds in Jefferson and Oldham counties in Kentucky

Figure 2-2: Plot and site design for sampling adult trees $(\geq 2.54 \mathrm{~cm} \mathrm{DBH})$, saplings, tree seedlings, and shrubs.

Figure 2-3: Silhouette plot of a cluster analysis resulting in three groupings of research sites....

Figure 2-4: Bank height measurements for land-use categories determined at the subcatchment scale. 74

Figure 2-5: Tree diameter distributions by land-use category in riparian zones 75

Figure 2-6a-b: Tree seedling richness/ $\log$ (Area sampled) for (a) near-stream plots only ( $\mathrm{n}=41$ nearstream plots), and (b) sites ( $\mathrm{n}=41$ sites $)$......

Figure 2-7: Square root of the tree density averaged across all nearstream plots in each land-use category

Figure 2-8: Shannon's equitability index $\left(E_{h}\right)$ for all woody species using nearstream plots only for comparisons.

Figure 2-9a-b: Exotic shrub species richness (a) and density (b) proportions with increasing impervious surface (subcatchment scale)......79

Figure 2-10: Partana ratios and overall silhouette widths for 2 to 10 clusters using optimal partioning.

Figure 2-11: Silhouette plot of cluster analysis using optimal partitioning and Bray-Curtis distance resulting in five groupings of research sites. 
Figure 2-12: 3 -dimensional configuration (only 2 dimensions shown) of woody plant data using non-metric multidimensional scaling (NMS) with sites (points) assigned to their respective groupings from the 3 -cluster configuration from cluster analysis

Figure 2-13: 3-dimensional configuration (only 2 dimensions shown) of woody plant data using non-metric multidimensional scaling coupled with cluster assignments from the 5-cluster configuration from cluster analysis.

Figure 2-14a-b: Non-metric multidimensional scaling of woody plant data coupled with cluster assignments from the 3-cluster configuration using cluster analysis, shown as colored shapes...

Figure 2-15a-b: Non-metric multidimensional scaling of woody plant data coupled with cluster assignments from the 5-cluster configuration using cluster analysis

Figure 2-16a-b: Non-metric multidimensional scaling of research sites arranged according to woody species composition.

Figure 2-17: Linear regression of $L$. maackii relative importance value (IV) against proportion of impervious surface within a radius of $500-\mathrm{m}$ of site centers ....................................................... 87

Figure 2-18a-b: Regressions of tree seedling and sapling densities compared to Lonicera maackii density at each site. .88

Figure 2-19: New conceptual model of variables that affect and are affected by vegetation within a riparian area modified from Figure 1-1....89

Figure 3-1: Schematic map of the three watersheds in Jefferson and Oldham counties in Kentucky. 135

Figure 3-2a-b: Plot and site structure for sampling adult trees $(\geq 2.54 \mathrm{~cm} \mathrm{DBH})$, saplings, tree seedlings, and shrubs.

Figure 3-3: Silhouette plot of a cluster analysis (complete clustering using Bray-Curtis distance) resulting in three groupings of research sites.

Figure 3-4: Groundcover species richness variation in nearstream plots with $\%$ impervious surface at the subcatchment scale.

Figure 3-5a-c: Box-and-whisker diagrams of diversity indices computed for groundcover species in urban, suburban, and rural riparian zones. 
Figure 3-6a-c: Relationship between diversity measures of groundcover species at riparian plots along first order streams and \% impervious surface cover within 1000 -m of each plot.

Figure 3-7: Mean groundcover riparian species richness at indicated distance intervals from the streambank in urban, suburban, and rural riparian zones

Figure 3-8: Linear regression model of cumulative catchment area upstream of the subcatchment for each suburban nearstream plot against normalized groundcover species richness

Figure 3-9: Non-metric multidimensional scaling (NMS) 3-dimensional configuration of research sites arranged by groundcover species composition.

Figure 3-10a-d: Non-metric multidimensional scaling (NMS) 3-dimensional configuration of research sites arranged by groundcover species composition and color-coded by cluster assignments from the 3-cluster configuration using cluster analysis....

Figure 3-11: Non-metric multidimensional scaling of research sites arranged according to groundcover species composition

Figure 3-12a-b: Scatterplots of Euonymus fortunei percent cover in response to Lonicera maackii density...

Figure 3-13a-b: Species richness (a) and Shannon's diversity (b) of groundcover species in nearstream plots in response to Lonicera maackil density

Figure 3-14: Shannon's equitability index of groundcover species in nearstream plots in response to Euonymus fortunei percent cover

Figure 3-15a-c: Shannon's Index (a), Simpson's Index (b), and species richness (c) of groundcover species in nearstream plots in response to Euonymus fortunei percent cover.

Figure 3-16: New conceptual model of variables that affect and are affected by vegetation within a riparian area modified from Figure 1-1.....154

Figure 4-1a-b: Air temperature and soil temperature taken during twice-monthly gas sampling visits in urban, suburban, and rural riparian sites in 2008 . 
Figure 4-2: Gravimetric soil moisture in the upper 15-cm soil horizon in urban, suburban, and rural riparian sites in 2008 ....

Figure 4-3a-b: Nearstream depth to groundwater (water table) in relation to bank height in years 2007 (a) and 2008 (b)... 198

Figure 4-4: Nearstream depth to groundwater (water table) from

November 2007 to October 2008.

Figure 4-5: Mean nitrate + nitrite- $\mathrm{N}$ nearstream riparian groundwater concentrations in urban, suburban, and rural subcatchments. 200

Figure 4-6: Mean ( $+\mathrm{SE})$ riparian $\mathrm{CO}_{2}$ flux between January and October 2008 averaged for sites in rural, suburban, and urban subcatchments .201

Figure 4-7: Principal components analysis (PCA) of sites and monthly $\mathrm{CO}_{2}$ fluxes.

Figure 4-8: Relationship between riparian winter $\mathrm{CO}_{2}$ flux and

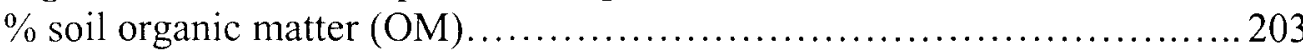

Figure 4-9: Relationship between riparian spring $\mathrm{CO}_{2}$ flux and soil

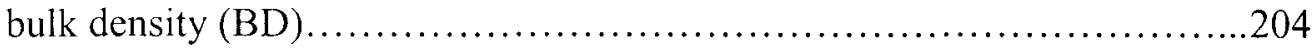

Figure 4-10: Increasing mean fall $\mathrm{CO}_{2}$ flux rates with increasing mean soil temperature among riparian sites.

Figure 4-11: Mean $( \pm \mathrm{SE})$ riparian $\mathrm{CH}_{4}$ fluxes from January to October 2008 averaged for sites in rural, suburban, and urban subcatchments........206

Figure 4-12: Principal components analysis (PCA) of sites and mean monthly $\mathrm{CH}_{4}$ fluxes from January to October 2008

Figure 4-13a-b: Mean monthly methane flux rates at each site from 2008 in relation to PCA row 1 scores from Fig. 13 for the months October (a) and September (b).....................................208

Figure 4-14: Changes in mean summer $\mathrm{CH}_{4}$ flux in relation to subcatchment $\%$ IS.

Figure 4-15: Changes in mean spring $\mathrm{CH}_{4}$ flux with increasing mean soil temperature among sites averaged over April and May 2008.

Figure 4-16: Summer mean net $\mathrm{CH}_{4}$ uptake (values less than zero) increases (values from left to right) with decreasing soil \% carbon. 
Figure 4-17: Variation in site $\mathrm{CH}_{4}$ flux with soil moisture................212

Figure 4-18a-c: Relationships between mean fall $2008 \mathrm{CH}_{4}$ flux and soil molar $\mathrm{C}: \mathrm{N}$ ratios (a) and gravimetric soil moisture (b) ...............213

Figure 4-19: Nitrous oxide flux from static chambers along riparian sites in three watersheds spanning an urban-to-rural gradient..............214 


\section{CHAPTER 1: INTRODUCTION: THE ECOLOGICAL STRUCTURE AND}

\section{FUNCTION OF RIPARIAN ZONES}

\section{Riparian zone vegetation}

Stream riparian zones are ecotones at the interface between terrestrial and aquatic systems. Therefore, width of the riparian zone, its plant communities and ecosystem processes are influenced by stream size, geomorphology, watershed drainage patterns and conditions in the adjacent stream channel as well as those in the upland catchment and upstream tributaries, (Fig. 1-1) (Dunne and Leopold 1978, Kalliola and Puhakka 1988, Naiman and Decamps 1997, National Research Council 2002). Riparian plant species survival and establishment can also be affected by biological factors that occur within the riparian zone, such as herbivory and competition for limiting resources (Naiman and Decamps 1997). However, flooding and sedimentation processes have been found to be the major determinants of the distribution of plant species in riparian areas (Robertson et al. 1978, Nilsson 1981, Hupp 1982, Kalliola and Puhakka 1988, Naiman and Decamps 1997).

Floodwaters not only disturb vegetation, but also deposit seed and other reproductive plant parts to downstream locations (Bendix and Hupp 2000). Nilsson et al. (1991) demonstrated the importance of water regimes in colonizing sites with seeds, suggesting that rivers can be significant corridors for some species of plants. For example, a regulated river exhibited the same riparian plant species richness as a non- 
regulated river, but differed in plant species frequencies. Varied hydrological regimes can also alter success of seed dispersal and plant establishment. Greater flooding frequency may allow deposition of a greater number of water-vectored propagules. Studies within riparian wetlands in southeastern Alaska have shown that areas with intermediate flood disturbance supported more species than either permanently or rarely flooded sites (Pollock et al. 1998). As stream size increases, the areal extent of its influence on the land and vegetation also increases.

\section{Riparian zones: role in the environment}

Riparian systems and their vegetation can also affect the adjacent stream, influencing hydrological dynamics and chemistry, stream temperature, sediment load, and organic matter inputs (Fig. 1-1) (Naiman and Decamps 1997). Riparian zones filter nutrients and sediment from surface and ground-water flowing through them towards streams (Brenner et al. 1991, Gilliam 1994), thereby mitigating eutrophication of stream systems. Eutrophication of water bodies can have detrimental effects on water quality, which riparian vegetation buffers may act to mitigate. Vegetation in the riparian zone reduces the velocity and erosion potential of floodwater moving over the floodplain and prevents sediment from entering the stream (Wenger 1999, Easson and Yarbrough 2002, Geyer et al. 2003, Boothroyd et al. 2004). Plant litter, debris dams, and uprooted trees provide nutrients and habitat for stream organisms (Naiman and Decamps 1997, Wenger 1999). Trees and shrubs provide shade, benefiting light sensitive and shade-tolerant riparian plants as well as stream organisms that depend on relatively cool stream temperatures for survival (Kiffney et al. 2004). Therefore, riparian environments provide 
many ecosystem services for society (Gregory et al. 1991) by improving quality of water entering streams, maintaining stream secondary productivity and therefore providing many recreational and human health benefits.

Riparian zones can act as effective sediment filters and nutrient sources or filters (Jacobs and Gilliam 1985a, Cooper et al. 1987, Lowrance et al. 1988) (Cooper et al. 1987, Puckett 2004). Riparian soils, since they lie adjacent to streams, have a water table relatively close to the soil surface. Coupled with periodic flooding, soil microorganismal processes can change in response to these moisture conditions based on the presence or absence of oxygen (Steudler et al. 1989). In addition to common soil functions that include nitrification, $\mathrm{N}$-mineralization, and soil respiration, riparian soil functions can shift towards lesser or greater soil nitrate $\left(\mathrm{NO}_{3}{ }^{-}\right)$and nitrite $\left(\mathrm{NO}_{2}{ }^{-}\right)$consumption, or methane production or consumption based on soil moisture. Greater soil saturation would result in increases in the anaerobic processes of denitrification and methanogenesis. Consumption of soil $\mathrm{NO}_{3}{ }^{-}$and $\mathrm{NO}_{2}{ }^{-}$and production of $\mathrm{N}_{2} \mathrm{O}$ and $\mathrm{N}_{2}$ gases would result in removal of soil nutrients, but potentially contribute to atmospheric greenhouse gas concentrations if $\mathrm{N}_{2} \mathrm{O}$ to $\mathrm{N}_{2}$ production ratios increase. Under aerobic conditions and when nitrification processes are high, however, $\mathrm{N}_{2} \mathrm{O}$ efflux from soils can be correspondingly high due to the "leaky pipe" phenomenon described by Davidson et al. (1993). Wet soils would also result in greater rates of methanogenesis, an anaerobic process producing the greenhouse gas, methane $\left(\mathrm{CH}_{4}\right)$. However, overall rates would depend on substrate availability and gaseous diffusion rates into and through the soil (Dorr et al. 1993, Schimel et al. 1993). The opposing process of soil $\mathrm{CH}_{4}$ uptake by methanotrophic bacteria could dominate net $\mathrm{CH}_{4}$ fluxes when soils are drier. 


\section{Riparian zones: effects of impervious surfaces}

Urban and suburban land cover and land uses, both upstream and adjacent to a particular riparian stretch, can influence stream characteristics, riparian vegetation cover, and plant species composition (Fig. 1-1). Impervious surfaces in the catchment are largely responsible for the environmental effects of urbanization on streams and their riparian areas (Arnold and Gibbons 1996). Urbanization results in less water percolation into the soil and greater overland runoff and conduit flow that bypasses many of the water quality and water absorption services provided by riparian soils and vegetation (Paul and Meyer 2001, Zipperer 2002, Meyer et al. 2005). As compared to rural lands, urban and suburban riparian areas may contain higher species diversity, since overland flow is more frequent and intense (Walsh et al. 2005) due to greater amounts of runoff from impervious surfaces. Since increasing impervious cover causes an increase in the amount and proportion of overland vs. belowground water flow and increases water entry through storm drains, streams in urban and suburban areas become characterized by higher amplitudes in stream flow volume during flooding (Walsh 2000). In nonmountainous and non-coastal urban areas, frequent and smaller high stream flow events can cause deeper incision of stream banks and scouring of the streambeds than larger, infrequent events (Neller 1989, Walsh 2000). As incision increases, the water table in the riparian zone drops to coincide with the new lowered level of the stream surface (Groffman et al. 2002, Groffman et al. 2003). Urbanized watersheds with highly incised streams have the potential to severely lower the riparian water table, which changes the dynamics of processes within the upper layers of the soil environment. Exacerbating this are local-scale edge effects created by urbanization, which also contributes to soil drying 
(Forman 1995, Zipperer 2002). As the amount of vegetation decreases within a riparian buffer, the amount of light that reaches the soil surface increases, which can alter the microclimate at the soil/atmosphere interface (Belnap et al. 2003). When this occurs, these once anaerobic, wet layers can more rapidly become aerobic, xeric layers, allowing organisms adapted to aerobic conditions to dominate the upper soil layers. This will therefore change nutrient cycling within riparian soils and, consequently, the chemical inputs from the riparian zone to the stream. Both lowered water tables and altered nutrient dynamics (i.e. carbon and nitrogen mineralization) can potentially change the types of plant species (e.g. wetland vs. upland species; proportion of nitrophilic species) that can persist within the altered riparian habitat. As the water table falls below the root zone, the ability of vegetation to sequester excess nutrients becomes more limited, thereby reducing the ecosystem services that they can provide (Groffman et al. 2002, Groffman et al. 2003, Groffman et al. 2004). Therefore, riparian vegetation and soil processes in urban and suburban areas are particularly important to study as they are key regulators of the aquatic-terrestrial linkage (Naiman and Decamps 1997).

\section{Urban influences on riparian vegetation}

Although impervious surfaces account for many of the environmental effects of urbanization on riparian vegetation, other urban influences on species diversity and exotic species composition are also important. These include increased fragmentation and edge effects, and shifts in abiotic and biotic influences from a highly altered matrix, including diverse and exotic seed sources from cultivated lots. Fragmented landscapes with a greater proportion of forest edges favor light-loving species over shade-tolerant species 
that tend to grow within forest interiors (MacDougall and Kellman 1992). For example, the abundance of exotic plant species in forests has been directly linked to increased road density and use, and subsequent increased light availability. Parendes and Jones (2000) found light availability to be strongly correlated to habitat type within the H. J. Andrews Experimental Forest, where sites along high-use roads had the greatest amount of light penetration. Consequently, the greatest numbers of exotic species occurred along these stretches of road. Canadian riparian areas studied along an urban-to-rural gradient showed a trend towards greater numbers of opportunistic species, which tended to be exotic annuals, within the urban riparian areas (Moffatt and McLachlan 2004). Vulnerable species, classified as perennial natives, occurred primarily at the rural end of the urbanization gradient in the Canadian study. Urban effects associated with density of humans can dramatically alter riparian vegetation dynamics. Species diversity and composition can be impacted by surrounding land use via seed dispersal from residential yards and other landscaped open spaces. Forest remnants and corridors in areas with higher densities of people could receive more plant propagules from landscaped areas, which tend to be exotics (Kendle and Forbes 1997, McKinney 2004). Areas with lower human density and fewer landscaped areas should be less vulnerable to these external plant inputs. Exotic, opportunistic species, therefore, may exhibit greater success within riparian areas that have been disturbed by such factors in the surrounding urban environment (Vidra et al. 2006). 


\section{Influence of proximate land use and riparian soil moisture on plant communities and soils processes}

Less understood factors related to riparian plant species dispersal and establishment include spheres of influence of different land-use types nearby (Fig. 1-1, Arrow 1), as well as differences in water table depths that differentially affect establishment (Fig. 1-1, Arrow 5a). Propagule dispersal and selection pressures on plant establishment differ for riparian and upland species, and depend on various spatial and environmental factors. Woody canopy species are more greatly influenced by macroscale environmental factors, while understory herbaceous and groundcover species are more greatly influenced by micro-scale environmental factors (Guillaume 2002). Also, with increasing forest fragmentation in urban areas (Zipperer 2002), corridors promoting propagule dispersal for native forest species may become limited, thereby reducing the native species pool. Increased forest edge density and lower riparian water tables in cities and suburbs could also differentially affect plant species establishment by changing light availability, nutrient cycling and surface soil moisture. These conditions could favor upland woody species that are better adapted to drier soils, or to soil moisture regimes that vary greatly intra- and inter-annually.

The interaction of urbanization and physical properties of the riparian zone can also result in different attributes of the plant community. Plant diversity has been shown to vary with location within riparian zones. Lateral movement away from and longitudinal movement downstream have both been shown to relate to species diversity

(Nilsson et al. 1994, Lite et al. 2005). A study of riparian plant species richness in arid environments in Arizona revealed decreasing herbaceous species richness moving 
laterally from the stream channel during a dry year (Lite et al. 2005). This finding was attributed to lower water availability farther from the stream, as the water table was found to drop with lateral distance from the channel. Furthermore, the opposite was true following monsoon rains and flooding, as herbaceous species richness increased with distance from the stream channel following increases in the water table. A difference in plant species diversity with lateral distance from the stream between riparian areas with varying land-uses is less well understood.

In addition, catchment area and longitudinal location along a stream from upstream to downstream has been shown to affect plant diversity. Nilsson et al. (1994) showed that a river in Sweden had a higher mean species richness along the main channel than the tributaries draining in to the channel. Explanations offered for differences in species richness were increases in mean annual discharge, and amount of peat and silt cover when comparing the main channel and its tributaries. In contrast, Lite et al. (2005) found a pattern of increasing mean plant species richness in an upstream direction following summer monsoon and flooding events in an arid environment as herbaceous annual species rapidly responded to limiting resource availability. Whether these patterns exist across a land-use gradient with changing resource availabilities and disturbances has yet to be determined.

Riparian functional responses to urban and suburban land-use contexts that require further study include gas flux rates and soil processes in relation to urbanization and water table depths. These functional responses are important, because they determine whether riparian zones are sources or sinks for particular nutrients and greenhouse gases. As more people move into growing urban areas, these processes will grow ever more 
important to climate modelers and land managers to formulate appropriate water management plans and atmospheric greenhouse gas predictions.

\section{Riparian projects within this dissertation}

I conducted three projects to determine riparian vegetation structure and gas flux dynamics along an urban-to-rural land-use gradient within three adjacent watersheds. The first project involved characterizing woody riparian plant communities and statistical explorations of factors likely to influence species composition of those communities. I did the same with the second project using herbaceous and groundcover species (woody vines) to determine the differing effects of site environmental variables on their abundance and diversity. In addition, the influences of impervious surface and National Land Cover Dataset land-cover types were explored at varying buffer distances around the sites. I also wanted to determine the correlations between defining species of the groundcover layer and those within the woody vegetation communities. The final project was aimed at evaluating the effect of urbanization on riparian functions by assessing soil gas flux responses to land-cover variables, soil nutrients, water table depth, and soil moisture differences.

The overarching goal of the first study was to determine how woody plant communities along streams in Louisville, Kentucky varied with degree of urban and suburban development in three watersheds. I accomplished this through a series of four objectives. The first objective was to determine differences in woody vegetation assemblages and investigate whether relationships with impervious surface cover and National Land Cover Database (NLCD) land-cover types could be detected at different 
spatial scales. The second objective was to determine whether differences in diversity and identity of plant species could be related to this urban-to-rural land-use gradient. The third objective was to determine the extent to which native and non-native woody vegetation was associated with the various land-use and -cover types in these catchments. The final objective was to determine the extent to which wetland and upland species were associated with various land-use and -cover types.

The overarching goal of the second study was to determine how groundcover (herbs and vines) plant communities along streams vary with degree of urban and suburban development in three watersheds in Jefferson and Oldham counties in Kentucky. I proposed a series of five objectives to accomplish this goal. The first objective was to determine whether potential relationships existed between riparian groundcover vegetation and variation in impervious surface cover and NLCD land-cover types at different spatial scales. The second objective was to determine whether differences in plant species diversity could be related to changes in this urban-to-rural land-use, to increasing catchment area and to increasing lateral distance from the streambank. The third objective was to determine the extent to which native and nonnative groundcover vegetation was associated with varying land-use and -cover types. The fourth objective was to determine the extent to which wetland and upland species were associated with various land-use and -cover types. The final objective was to determine whether groundcover species associations and diversity differed with the degree of colonization by the exotic shrub, Lonicera maackii.

The primary goal of the third study was to assess variation in soil gas fluxes in riparian soils in relation to soil nutrients, moisture, groundwater depth, and temperature. 
Since depth to water table and soil moisture content is a known determinant of soil gas flux, the first objective, was to determine whether differences in nearstream water table depth existed in urban, suburban, and rural riparian zones. I predicted that urban riparian water tables would be lower when compared to suburban and rural reference riparian sites because of higher streambanks (see Dissertation Chapter 2). My second objective was to measure flux rates of three greenhouse gases (carbon dioxide $\left(\mathrm{CO}_{2}\right)$, methane $\left(\mathrm{CH}_{4}\right)$, nitrous oxide $\left.\left(\mathrm{N}_{2} \mathrm{O}\right)\right)$ from urban, suburban, and rural riparian zones. Assuming that plant root density and organic matter content and lability in riparian soils varied similarly along the land-use gradient, I predicted that $\mathrm{CO}_{2}$ flux could be higher in urban areas due to the "urban heat island" effect whereby higher atmospheric temperatures would raise soil temperatures, thereby increasing microbial activity and root respiration. In addition drier soils should favor aerobic microbial activity over that of anaerobes, with the consequence that $\mathrm{CO}_{2}$ efflux should dominate over $\mathrm{CH}_{4}$ efflux. I also predicted that net methane uptake (methanotrophy) would occur within urban riparian sites, as urban soils were predicted to be driest among the three land-use types and methanotrophs are obligate aerobes. Conversely, I predicted a net methane efflux (methanogenesis greater than methanotrophy) from soils in rural reference sites, because shallow water tables and subsequent anoxic soil should favor methanogens. Since denitrification is also an anaerobic process, I predicted the greatest $\mathrm{N}_{2} \mathrm{O}$ flux rates would occur in rural reference sites due to the shallower water table and higher soil moisture. However, because $\mathrm{N}_{2} \mathrm{O}$ efflux from soil can also occur under aerobic conditions when nitrification rates are high, the direction and magnitude of the gradient effect on this process may not be as predictable. My third objective was to evaluate the influence of groundwater nitrate and 
dissolved organic carbon (DOC) concentrations on $\mathrm{N}_{2} \mathrm{O}$ flux rates, because denitrifying bacteria depend on DOC as an energy source and $\mathrm{NO}_{3}{ }^{-}$as a final electron acceptor. Therefore, I predicted higher concentrations of groundwater DOC and nitrates would result in greater nitrous oxide flux rates from the soil under anaerobic conditions. The fourth objective was to evaluate the influence of soil properties on greenhouse gas flux rates. I predicted \% clay and soil bulk density would be negatively correlated with $\mathrm{CH}_{4}$ uptake due to their impact on gas diffusion rates into and through the soil from both deeper soil horizons and from $\mathrm{CH}_{4}$ in the atmosphere. I also predicted flux of $\mathrm{CO}_{2}$ and $\mathrm{N}_{2} \mathrm{O}$ would follow patterns others have found with regards to bulk density (Xu and Qi 2001), with decreasing flux rates at higher bulk densities due to fewer soil pore spaces for microbial activity. 


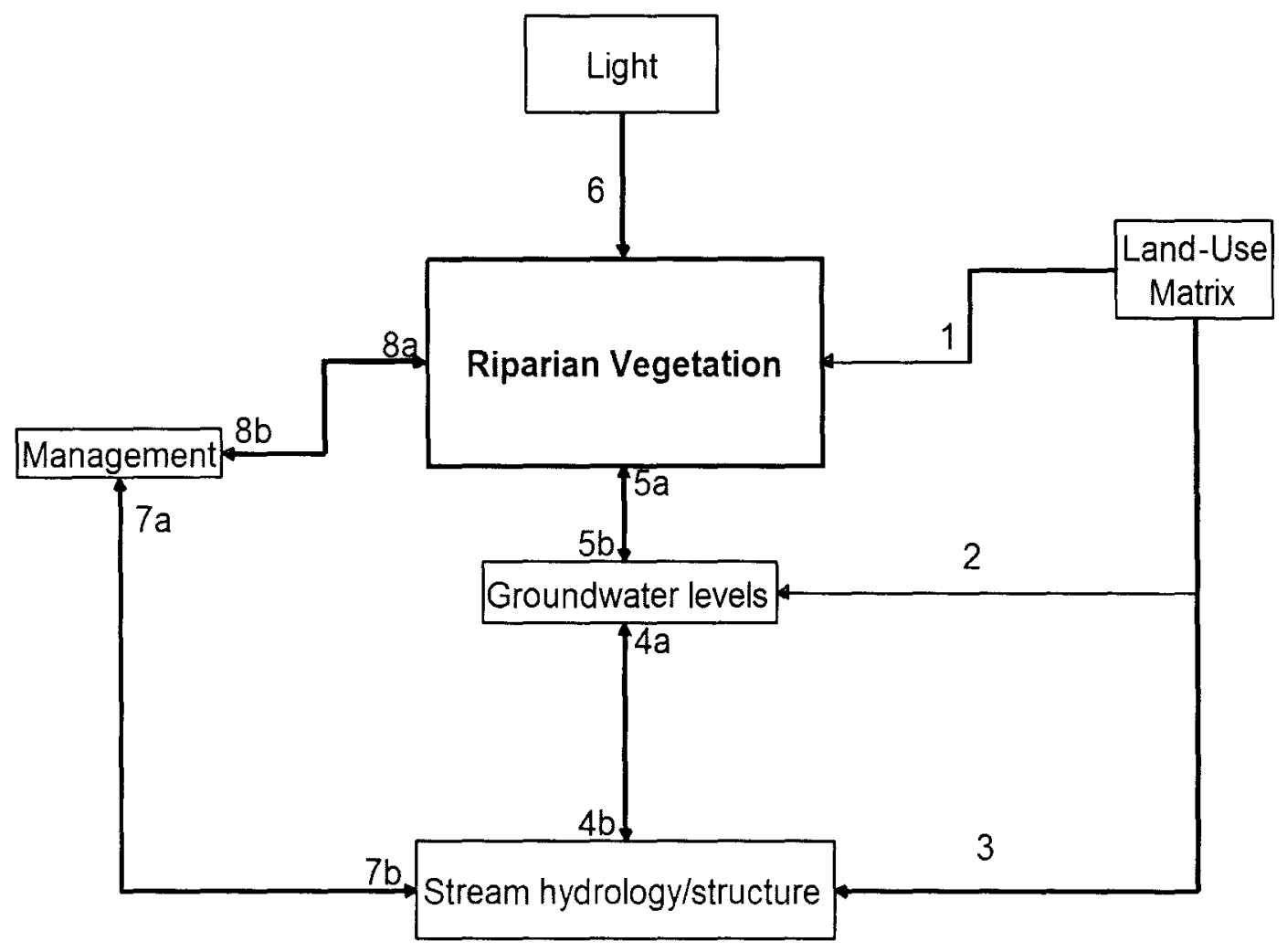

Figure 1-1: Conceptual model of variables that affect and are affected by vegetation within a riparian area. In this study, I will focus on the arrow labeled 1 . The land-use matrix can affect riparian vegetation both directly (arrow 1) and indirectly (arrows 2 and 3 ), as illustrated. Stream hydrology and structure affect groundwater levels (arrow 4a), thereby indirectly affecting vegetation. Groundwater levels feed back on stream hydrology by determining stream depths and determining whether particular reaches are gaining or losing water (arrow 4b). Groundwater level can be altered through processes such as transpiration and degree of overland water flow and can determine which vegetation types can survive, depending on their root tolerance for wet, microaerophilic conditions (arrows 5a and 5b). The vegetation structure can affect the amount of light that permeates to the ground, and light affects the type of subcanopy vegetation that can grow based on light tolerance (arrow 6). Management decisions may be based on structure and/or water levels within the stream (arrow 7a), and some management decisions can affect stream levels and structure (arrow 7b). Highly incised streams have lowered water tables within the riparian zone. Management decisions or lack of management can determine dominant vegetation types (arrow 8a). Riparian species may be preferred over drier-adapted species when attempting to restore a riparian zone to a more natural vegetative state. Restoration management decisions are based on existing vegetation (arrow 8b). 
CHAPTER 2: VARIATION IN WOODY STREAMBANK PLANT COMMUNITIES ALONG URBAN, SUBURBAN, AND RURAL STREAMS IN METRO LOUISVILLE, KENTUCKY

\section{Introduction}

Riparian zones adjacent to streams perform a number of valuable ecosystem services. As ecotones between terrestrial and aquatic systems, riparian areas act as sediment and nutrient filters (Brenner et al. 1991, Gilliam 1994), provide habitat and nutrients to stream and riparian organisms (Naiman and Decamps 1997, Wenger 1999), and reduce floodwater velocity and erosion potential (Wenger 1999, Easson and Yarbrough 2002, Geyer et al. 2003, Boothroyd et al. 2004). Riparian woody vegetation plays a significant role in providing these services (Brenner et al. 1991, Gilliam 1994, Naiman and Decamps 1997). Since riparian zones occur adjacent to streams and rivers, reductions of or alterations to these services would affect water quality, resulting in reduced recreation potential for swimming and fishing, and enhanced water treatment services and costs to towns and cities using these water sources for drinking. Because urban areas are growing and becoming more populous (Cordell and Macie 2002), the need to protect water resources from the effects of development will increase. Since woody vegetation within riparian zones provides such valuable services, it deserves particular attention to determine in what ways species structure will change as the land becomes more developed. In addition, depending on their sensitivity to land-use change, 
riparian vegetation may also serve as an indicator of environmental change (Naiman and Decamps 1997).

Riparian woody species are more greatly influenced by macro-scale anthropogenic disturbances due to forest and river management rather than micro-scale influences such as site-level soil nutrient and moisture differences (Guillaume 2002). Along a river system in Sweden riparian sites along the main channel had higher mean abundances of species than along smaller tributaries (Nilsson et al. 1994). Furthermore, the middle reaches of the main channel exhibited the highest species richness, which may have been related to flooding differences. Differential effects of flooding dynamics on woody species composition and diversity have also been observed in other systems. For example, studies within riparian wetlands in southeastern Alaska have shown that areas with intermediate flood disturbance supported more species than either permanently or rarely flooded sites (Pollock et al. 1998).

Some of the ways urbanization can alter the environment that could then affect riparian woody species composition include lowering of the water table via stream incision (Groffman et al. 2003), increasing the proportion of forested edges (Forman 1995, Zipperer 2002), and producing more intense, flashy floods (Paul and Meyer 2001, Walsh et al. 2005). Water table depth changes are important since woody species vary in their ability to tolerate high moisture, low oxygen soil conditions (Naiman and Decamps 1997). Conversely, chronically low water tables can stress those species requiring higher moisture levels and favor species able to tolerate dryer conditions (Groffman et al. 2003). Forest edges, while contributing to species diversity, also attract non-native species (Fraver 1994, Zipperer 2002) due 
to factors that include greater seed capture potential along forested edges (Cadenasso and Pickett 2001), higher light intensity and wind turbulence, greater temperature fluctuations, and lower soil moisture conditions (Gehlhausen et al. 2000, Zipperer 2002). Changes in flooding regimes can affect riparian vegetation communities due to changes in minimum and maximum flows (Auble et al. 1994, Naiman and Decamps 1997). These changes can result in reduced establishment and resource conditions that favor specific woody species over others (Bren 1992, Johnson 1993, Naiman and Decamps 1997). All of these changes can occur in predictable ways along an urban-to-rural gradient, resulting in distinct woody plant communities.

The overarching goal of this study was to determine how woody plant communities along streams in Louisville, Kentucky vary with degree of urban and suburban development in three watersheds. The first objective was to describe the woody vegetation assemblages and investigate whether relationships with impervious surface cover and National Land Cover Database (NLCD) land-cover types could be detected at different spatial scales. Specific predictions developed under this objective were that distinct woody vegetation communities existed that could be correlated with impervious surface cover, as an index of urbanization. The second prediction was that the sphere of influence of different land-use and land-cover types on woody vegetation communities would be scale-dependent and correlate more strongly at larger scales due to the relatively low magnitude of woody species response to microclimatic conditions (Guillaume 2002). The second objective was to determine whether predictable differences in diversity and identity of plant species existed along this urban-to-rural 
land-use gradient. I predicted that woody plant diversity would be higher in suburban riparian areas than in either urban or rural riparian ones, because of the presumed availability of more diverse seed sources from low-density residential areas. The third objective was to determine the extent to which native and non-native woody vegetation was associated with the various land-use and -cover types in these catchments. Since one consequence of urbanization is creation of edges along vegetation patches, I predicted that native vegetation would dominate riparian assemblages associated with forested rural land-cover, and that exotic species would take on greater importance in riparian assemblages associated with urban land-use types. The final objective was to determine the extent to which wetland and upland species were associated with various land-use and -cover types. Because of greater impervious runoff and stream incision observed in cities, I proposed that higher streambanks would be found in urban areas, and that streambank height would be positively correlated with species adapted to drier soil conditions, and negatively correlated with wetland species. I specifically predicted that obligate and facultative wetland species would assume greater dominance in riparian assemblages associated with forested rural land-cover and that facultative upland and upland species would exhibit greater dominance in riparian assemblages associated with urban landcover types.

\section{Methods}

Study Area

The study area consisted of three watersheds located within and adjacent to Louisville, Kentucky, USA (Latitude $38^{\circ} 15^{\prime} \mathrm{N}$, Longitude $85^{\circ} 46^{\prime} \mathrm{W}$ ), which has a 
population of approximately 700,000 with a mean density of 695 people $\mathrm{km}^{-2}$ (U.S. Census Bureau 2008). These were the Beargrass Creek (Middle Fork), Goose Creek, and Harrods Creek (South Fork) watersheds. The watersheds lie adjacent to each other with all streams flowing in a primarily westerly direction and eventually emptying into the Ohio River (Fig. 2-1).

The three watersheds are of approximately equivalent sizes, containing varying proportions of impervious surface cover (ISC; Table 2-1). Beargrass Creek watershed, located in the north-northeast portion of Jefferson County, KY, is approximately $65 \mathrm{~km}^{2}$ (Metropolitan Sewer District 1999b) and contains the greatest proportion of impervious surface cover (33\% ISC). This Middle Fork of Beargrass Creek joins the South Fork of Beargrass Creek before flowing into the Ohio River. Several stretches of this stream are featured on Kentucky's Division of Water 303d list, which list streams with impairment issues as well as the pollutant(s) causing the impairment (Kentucky Environmental and Public Protection Cabinet 2008). Since combined sewer overflows (CSOs) commonly occur in this stream, the presence of fecal coliform bacteria and sewage are stated as the problem pollutants within Beargrass Creek.

Goose Creek is a $50-\mathrm{km}^{2}$ watershed also in Jefferson County, in-between and adjacent to Beargrass and Harrods Creek watersheds (Metropolitan Sewer District 1999a). Of the three watersheds in this study, impervious surface cover is intermediate ( $20 \%$ ISC). The creek is composed of a pair of forks that meet before emptying into the Ohio River. Goose Creek is also listed on the $303 \mathrm{~d}$ list, with cadmium and fecal coliform levels stated as impairment issues. 
South Fork Harrods Creek watershed $\left(60-\mathrm{km}^{2}\right)$, which has the lowest impervious surface cover of the three ( $10 \%$ ISC), is located primarily in neighboring Oldham County, but extends into northeast Jefferson County. The South Fork of Harrods Creek eventually joins the North Fork of Harrods Creek to become Harrods Creek before flowing into the Ohio River. Sections of the larger Harrods Creek network were added to the $303 \mathrm{~d}$ list in years past, specifically the portion passing through Jefferson County, which is a more developed area. As in the other two watersheds, fecal coliform levels have been a persistent problem (Metropolitan Sewer District 1999c). The current 303d list contains Harrods Creek with fecal coliforms as an impairment issue, although this information is for the stream section downstream of where the North Fork and the South Fork Harrods Creek join.

\section{Watershed classification}

Watersheds were chosen from digitized catchment layers downloaded from the Louisville/Jefferson County Information Consortium (LOJIC) database (LOJIC 2010). LOJIC is a multi-agency institution that maintains a geographic information systems (GIS) database to serve primarily Jefferson County, KY, although limited information is available for a portion of Oldham County, KY. After checking for topographical accuracy using topographical maps of the watersheds and ArcGIS 9.0 software, I divided watersheds into subcatchments based on stream order and topography from the original LOJIC layers. I further divided subcatchments of higher stream orders with longer reaches into two or three constituent subcatchments based on topographic boundaries. 
Beargrass Creek watershed contained 13, Goose Creek watershed 12, and Harrods Creek watershed 14 subcatchments.

Impervious land-cover classification was determined using three data layers from the LOJIC database digitized in 2001: buildings, miscellaneous transportation, and roads. The buildings-layer included all built structures, whether commercial or residential. The roads-layer included all paved road surfaces, excluding parking lots. The miscellaneous transportation-layer included secondary transportation features such as driveways, parking lots, and sidewalks. The area of land covered by impervious surfaces for each subcatchment was determined using ArcGIS 9 (ESRI, 2010) and was divided by the total land area for each subcatchment to determine the proportion of impervious surfaces.

Categorization of subcatchments into urban, suburban, and rural land-use types was modified from Schuler (1994). For this study, urban subcatchments were defined as containing $\geq 30 \%$ impervious surface cover. Rural subcatchments contained $\leq 10 \%$ and suburban subcatchments between $10 \%$ and $30 \%$ impervious surface cover. Research sites were classified by land use based on impervious surface cover at three scales. The first and largest areal scale was the subcatchment level, where sites were classified as urban, suburban, or rural based on the \% impervious cover for subcatchment in which they were located. Impervious surface cover was calculated at two additional smaller scales surrounding the sites. Site buffers were created using ArcGIS 9 at a radius of 1-km and $500-\mathrm{m}$ from the site center. The proportion of impervious surface cover was determined for these buffers in the same way as for the subcatchments. Sites were then classified into one of the three land-use categories in the same way as for the 
subcatchment scale of analysis. Therefore, urban, suburban, and rural categorization of sites was changed depending on the scale at which impervious cover was calculated.

\section{Site selection and plot establishment}

Sites for plot establishment were chosen among riparian zones within the aforementioned subcatchments. A vertical line was drawn the length of the stream section within the subcatchment using ArcGIS 9.0 software and a digital map of the watersheds. A random number multiplied by 100 meters was used to find the location of the site along the vertical measurement line, starting at the downstream end. Sites were visited and chosen if they were at least partially covered with unmanaged vegetation and at least 100 meters from the nearest fork to avoid sampling riparian zones from separate subcatchments. Riparian zones were defined by topography and structural confinements, such as roads. Areas of level ground between the streambank and either an upland slope, structural barrier (roads, parking lots, buildings), or other obstruction (grazed pasture, ephemeral stream channel) were considered as being within the riparian zone. Riparian zones located completely within regularly used pastures and those found to be mown to the edge of the streambank were not used for this study. Only one side of the stream was sampled at each site. If both sides contained vegetation, the larger side was chosen, if it fit the criteria above, if it was reachable, and if the proper permissions could be attained. If only one side of the stream contained vegetation then that side was chosen. Locations where at least one full plot could not be placed (riparian width $<12-\mathrm{m}$ ) were sampled with a partial plot due to the difficulty in acquiring entry permission at many of the sites, and to avoid potential biases of purposefully choosing a site of a particular width. The 
distribution of riparian widths by stream order and land-use category is provided in Table $2-2$

After visiting the locations identified by ArcGIS selection, the location of the actual study plot or plots was chosen by taking a randomly determined number of steps parallel to the stream to establish the center of a transect. A compass was used to extend a transect tape perpendicular to and away from the stream channel up to 91 meters, if possible. Circular plot centers (plot radius $=5.64-\mathrm{m}$, plot area $=100-\mathrm{m}^{2}$ ) were established beginning at 6,46 , and 86 meters perpendicularly away from the stream channel. Three belt transects measuring $10 \times 2$ meters were nested within the circular plots, with the long edge perpendicular to the stream. The distance between midlines of adjacent belt transects was four meters. Twelve $1 \times 1$ meter quadrats were nested within the belt transects (four quadrats per belt transect). Quadrats were placed every two meters down the midline of each belt transect, so that the centers of adjacent quadrats within a belt transect were three meters apart (Fig. 2-2). Trees $>1 \mathrm{~m}$ high and $\geq 2.54 \mathrm{~cm} \mathrm{DBH}$ were measured within the circular plots, living stem counts of shrubs and saplings ( $>1-\mathrm{m}$ high, $\leq 2.54-\mathrm{cm} \mathrm{DBH})$ were measured in the belt transects, and tree seedlings were measured in the $1-\mathrm{m}^{2}$ quadrats.

For each 100- $\mathrm{m}^{2}$ plot, I determined the diameter at breast height (DBH) and number of trees by species of all trees $>2.54-\mathrm{cm} \mathrm{DBH}$. Trees were determined to be inside or outside of the circular plot using a ny-pole (Nyland and Remele 1973). This was a wooden pole wide enough to be seen clearly from 6 meters. Two horizontal lines were positioned at eye level using different colored tape so that they overlapped flush when viewed through a prism (10 BAF) held at 5.64 meters from the pole. Standing next to 
each tree, one edge of the prism was used to view the ny-pole (positioned in the plot center). If the lines on the ny-pole did not overlap or overlapped slightly, then the tree was counted within the boundary. If the lines overlapped flush with each other, then the tree was considered at the boundary of the plot, and every other tree in that category was counted. If the lines extended past each other, so that the top line appeared on the bottom and the bottom line appeared on the top, then the tree was outside the boundary of the

plot and was not counted. For each $20-\mathrm{m}^{2}$ belt transect, shrub stem number $(>1-\mathrm{m}$ high) and sapling ( $>1$ meter high, $<2.54-\mathrm{cm} \mathrm{DBH)} \mathrm{number} \mathrm{was} \mathrm{recorded} \mathrm{for} \mathrm{each} \mathrm{species}$ using a meter stick held perpendicular to the transect tape with one end held directly over the tape. Any stem occurring beyond the far end of the meter stick was not counted. For tree and shrub seedling $\left(<1-\mathrm{m}\right.$ high) counts and their respective $\%$ cover, four $1-\mathrm{m}^{2}$ quadrats were nested two meters apart within each of the three $20-\mathrm{m}^{2}$ belt transects $(12$ quadrats per circular plot). For all vegetation data, only livings stems were considered. Tree, sapling, and shrub sampling was conducted in 2005 (June through August) and in 2006 (May to October). Tree seedling samples were made in 2006 (May - October).

\section{Plant identification and classification}

Plant identification was conducted in the field whenever possible. The primary source used for field identification was Wharton and Barbour (1973). Plants that could not be identified in the field were collected for later keying using Jones (2005). Collections included as many parts of the plant as possible, including stem, leaf, and flowering parts. However, the authority for all species names used here was the USDA PLANTS Database (2010). Native status at the continental level was determined using 
Jones (2005) and the USDA PLANTS Database (2010). Three categories of species provenance at the continental scale were used: native, exotic, and naturalized. Term usage is as defined in the USDA Natural Resources Conservation Service (2009). Native plants are those that have developed prior to European settlement in an area (in this instance, the continental U.S.). Exotic plants are those introduced with human assistance to a continent on which they were not previously found during the pre-European era. Naturalized species are exotic species that do not need human assistance to successfully reproduce and establish, thereby maintaining populations over an unspecified period of time. Inclusion of the naturalized species category was to distinguish exotic species in urban and/or suburban areas that were ornamental species likely coming from yards or cultivated landscapes.

\section{Importance value and diversity}

Relative importance value (IV) for each tree species $(>2.54-\mathrm{cm} \mathrm{DBH})$ was calculated at each site (Appendix 1) and for each land-use category (Table 2-3). For analytical purposes, the term 'site' refers to the combination of all plots at a particular research location, to distinguish from those instances when only the plot nearest the stream was used. The IV at the site level was calculated as the sum of relative density ((Stem density for a single species (stems/ha)/Total stem density for all species $\left.(\text { stems } / \text { ha })^{*} 100\right)$ and relative dominance ((Basal area for single species $\left(\mathrm{m}^{2} / \mathrm{ha}\right) /$ Total basal area for all species $\left.\left.\left(\mathrm{m}^{2} / \mathrm{ha}\right)\right)^{*} 100\right)$. 
Eq. 1

Tree $(>2.54-\mathrm{cm} \mathrm{DBH})$ species \% IV $=\left(\left(\right.\right.$ species stems ha $\left.{ }^{-1}\right) /\left(\right.$ total stems ha $\left.{ }^{-1}\right)+($ species

$$
\left.\left.\mathrm{m}^{2} \mathrm{ha}^{-1} / \text { total } \mathrm{m}^{2} \mathrm{ha}^{-1}\right)\right) * 100
$$

Sapling ( $>1-\mathrm{m}$ high, $<2.54-\mathrm{cm} \mathrm{DBH})$, tree seedling $(<1-\mathrm{m}$ high) and shrub IV at the site level was calculated from relative density $\left(\left(\right.\right.$ species stems $\left.\mathrm{ha}^{-1}\right) /\left(\right.$ total stems $\left.\left./ \mathrm{ha}^{-1}\right)\right)$ and relative frequency (species occurrence per sampling unit [quadrats or belt transects]/total occurrences). For analytical purposes, only shrubs greater than one meter high were used to compute the IV's for each site, unless no shrubs of that species were present, in which case shrub seedlings $(<1-\mathrm{m}$ high) were used. This was done to reduce the sampling bias caused by collecting stem counts at different scales (quadrat vs. belt transect) due to the clumping nature of shrub growth.

Eq. 2

Sapling or Tree seedling or Shrub \% IV $=\left(\left(\right.\right.$ species stems ha $\left.{ }^{-1}\right) /\left(\right.$ total stems $\left./ h^{-1}\right)+$ (species occurrence per sampling unit/total occurrences)) $* 100$

Species diversity was expressed using species richness, Shannon's ( $\left.H^{\prime}\right)$ Index (Shannon 1948, Magurran 2004) and Simpson's Reciprocal (1/D) Index (Simpson 1949, Magurran 2004). 
Eq. 3

$H^{\prime}=\sum_{i=1}^{S}\left(p_{i} \ln p_{i}\right)$

Eq. 4

$\mathrm{D}=1-\frac{\sum_{i=1}^{5} n_{i}\left(n_{i}-1\right)}{N(N-1)}$

Where $\mathrm{S}=$ species richness, $p_{i}=$ proportion of an individual species, $n_{i}=$ number of stems for a species, and $N$ is the total number of stems. Shannon's equitability index $\left(E_{h}\right)$ was also computed as a measure of community evenness:

Eq. 5

$E_{h}=H^{\prime} / \ln (S)$, where $H$ is Shannon's index and $S$ is species richness.

Because of different riparian widths and plot sizes, richness was calculated for all sites as well as solely for nearstream plots as the number of species divided by the log of the area sampled (Conner and McCoy 1979, Nilsson et al. 1997). Species richness was calculated by totaling the species across all plots for a site, and in some other comparisons by considering richness in only the plot nearest the stream.

EstimateS (Colwell 2009) was used for computing diversity indices. Densities of woody species (trees, saplings, tree seedlings, and shrubs) were used to compute Simpson's reciprocal and Shannon's indexes for plots nearest the stream. Tree, sapling, and tree seedling information was collected at different spatial scales, so densities were computed separately for each life stage and added together where shared species existed 
across life stages. In EstimateS, diversity index estimators were not used, so indices were computed using the observed species order within the input file.

Metrics for wetland indicator status (WIS) and species provenance (NEZ; $\mathrm{N}=$ native, $\mathrm{E}=$ exotic, $\mathrm{Z}=$ naturalized) were determined for each site to assess correlations with various land-cover variables. Species were labeled with their WIS from the USDA PLANTS database as well as NEZ as described previously. WIS and NEZ codes and definitions are shown in Appendix 2. A numerical value was assigned to each site for each category of WIS and NEZ using the relative IV's calculated for each species in each site. Values were calculated by summing the IV's of the species in each category and adding a weighting factor to account for the number of species within that category. The weighting factor was the proportion of species within each category divided by the total number of species within the site. Being based upon relative IV's, the maximum value for any category, all others being zero, was 900 (200 each for trees, saplings, tree seedlings, and shrubs +100 for the weighting factor). For example, the relative IV for Acer negundo, a native tree, would be 200 if it were the only tree species in a site. If it was also the only sapling and seedling species located at that site then those IV's would also be 200. Since $A$. negundo is a native species the computed metric for the category "native" would be:

Tree IV + Sapling IV + Seedling IV + Shrub IV + Weighting factor [\# native spp./Total \# spp. $\left.{ }^{*} 100\right]=$ Native metric 
$200($ Tree IV $)+200($ Sapling IV) $+200($ Seedling IV $)+0($ Shrub IV) +1 native spp. $/ 2$ total spp. $* 100=650$

If a naturalized species, such as Lonicera maackii, were the only shrub, it would have an IV of 200. The computed metric for the category "naturalized" in that same plot would be:

Tree IV + Sapling IV + Seedling IV + Shrub IV + Weighting factor (\# nat. spp./Total \# spp. $\left.{ }^{*} 100\right)=$ Naturalized metric

$0($ Tree IV $)+0($ Sapling IV $)+0$ (Seedling IV $)+200($ Shrub IV $)+(1$ nat. spp/2 total spp. $)^{*} 100=250$.

Data analysis

Potential relationships between plant species composition, degree of imperviousness as well as other land-cover attributes, and other environmental parameters listed below were explored using non-parametric ordinations (PC-Ord v. 4.41) (McCune and Mefford 1999) and R v.2.11 software (R Development Core Team 2009), indicator species analysis, and cluster analyses (R v.2.11) (R Development Core Team 2009). Scatterplots were used to explore relationships between diversity and abundance measures of the woody vegetation strata with \% IS at the subcatchment scale. Non-metric multidimensional scaling (NMS) in PC-Ord was executed according to the recommended procedure outlined in McCune \& Grace (2002). For each analysis, a separate starting configuration was supplied based on an initial analysis with the following configuration: 
Sorensen distances, 50 runs with real and random data, a random starting configuration, and 200 iterations to evaluate stability of the final solution (stability criterion $\leq 0.0005$ ). Dimensionality was assessed using a Monte Carlo test for significance, with a p-value indicating the probability of finding the computed stress value for a specific number of dimensions by chance (based on 1000 permutations). Final solutions were completed using one run with real data. Ordinations run using $\mathrm{R}$ were given an optimal geometric starting configuration based on principal coordinates analysis.

The goal of cluster analysis was to obtain groupings of study sites based on commonalities in species composition. To do this, a site dissimilarity matrix was constructed based on woody species composition. Several dissimilarity measures and clustering techniques were applied to the data in an attempt to achieve the best cluster configuration for the sites. A clustering technique known as optimal partitioning was decided upon as it produced the highest within-to-among cluster similarity value (Partana ratio). To accomplish this, the Bray-Curtis distance measure was used to create a dissimilarity matrix of the sites prior to cluster analysis. To decide on the number of groupings and assess their overall quality, I used the Partana ratio, as mentioned, as well as silhouette plots. Figure 2-3 shows an example of a silhouette plot for three groupings. Silhouette plots assign numerical values termed "silhouette widths" $\left(\mathrm{S}_{\mathrm{i}}\right)$ to each site (i) with a value between -1 and 1 within a cluster. The silhouette width is obtained by comparing a site's mean similarity to other sites within the cluster, followed by comparison to its mean similarity to sites within the nearest cluster. A silhouette width of 1 means the within-cluster similarity is much higher than between-cluster similarity, indicating a good fit of that site to the cluster. A value of -1 means the between-cluster 
similarity is much higher than within-cluster similarity, indicating a poor fit of that site to the cluster. An average silhouette width is computed for each cluster to compare quality of the groupings. An overall silhouette width is computed for the whole graph to compare different numbers of groupings, where the goal is to obtain a value as high as possible without compromising the quality of the individual clusters.

Indicator species analysis (ISA) is a useful method for evaluating different species as indicators of environmental conditions. ISA is a statistical technique for species comparisons across two or more groups of sample units (sites, quadrats), taking into account abundance and frequency of each species within a group. An indicator value is assigned to each species in each group, and the value is tested for significance using a randomization procedure (Monte Carlo). Indicator values range from 0 to 1 , where ' 0 ' means the species is not present in the group and ' 1 ' means it is always present within and exclusive to that group (Dufrene and Legendre 1997).

To determine whether measures of environmental degradation (i.e., bank height) and measures of abundance and species diversity (stem density, diversity indices) varied with the proportion of land cover, a single factor model (ANOVA, R v.2.11) was used for partitioning variance among land-use categories (urban, suburban, rural). Tukeys HSD pairwise comparisons were used for determining statistical significance of the variance between land-use categories. Data were assessed for homoscedasticity and homogeneity of variance prior to analysis and were transformed to better meet those assumptions, if necessary. In addition, any potential outliers were identified using quantile by quantile (Q-Q) plots and graphs of leverage vs. standardized residuals plotted with Cook's distance. Points appearing to assert a disproportional influence on the data were 
sequentially eliminated from the analysis to assess effects on the statistical assumptions of homoscedasticity and homogeneity of variance. Data points were permanently removed from the analyses if their elimination improved these statistical assumptions.

\section{Environmental parameters}

Several environmental and socio-economic variables external to the plots were used to explore their ability to explain variation in plant species composition among plots. These were $\%$ impervious surface cover (at three scales: the subcatchment, within $1-\mathrm{km}$ radius and within a $0.5-\mathrm{km}$ radius of site center), canopy openness, bank height, plot size and distance from stream, stream order, cumulative upstream catchment area, and monetary value of residential property. Impervious surface cover was determined as described previously and the three scales were used to explore which scale better predicted plant community composition. Since canopy openness and hence light can also be a determinant of species presence and dominance, a spherical densiometer (Lemmon $1956,1957)$, held over the middle of each $1-\mathrm{m}^{2}$ quadrat at each plot, was used to determine this measure. These values were averaged to obtain percent canopy openness for each plot. Cumulative catchment area was determined by adding subcatchment areas upstream of the site to the area of the subcatchment in which the site was located. This was done because catchment size is believed to affect stream conditions including flood potential, and therefore, it could be important for riparian plant composition. Bank height was measured from the first terrace out of the channel and referenced to the depth at the middle of the stream bed. Where the center of the channel was not reachable from the bank or by wading, bank height was measured at arm's reach. Bank height was measured 
once at the midline of each plot nearest the streambank. These measurements were used as a proxy for depth to groundwater to determine correlations with species composition at the sites. Additional measurements were taken 50 meters to either side of the plot midline at 13 of the 41 sites where studies of depth to ground water and soil trace gas fluxes were conducted. These 13 additional measurements were averaged with the 41 site measurements to assess differences in overall bank height by land-use category.

To explore relationships between species identity, percent exotic species, and the measures of community diversity with a measure of socio-economic status of land owners, property value determinations were made from information at the Property Valuation Adminstrator offices in Jefferson and Oldham counties. The value of the parcel of land in which the site was located was taken as the property value. In instances where the site was located on the edge of a park abutting residential housing units or businesses, the average values of surrounding privately owned parcels was taken as the property value. Where sites were located in the interior of a park or natural area, the property was assigned a value of $\$ 1$ to indicate no private monetary value.

Finer-grained land-cover categorizations were also obtained using the U.S. Geological Survey's National Land Cover Database from 2001 (NLCD 2001; Appendix 3) acquired through LOJIC. NLCD 2001 is a database developed from a consortium initiated by the U.S. Geological Survey to interpret land-cover attribute categories from Landsat 5 and 7 images (Homer et al. 2004). Pixels from these images were assigned digitized land-cover attribute categories with a spatial resolution of $30-\mathrm{m}$. This digitized layer was used to determine the areal proportion of land represented by each land-cover category surrounding each study site within two circular areas of different sizes (1-km 
and 500-m radius buffers). The proportions of these land-cover categories were entered as environmental variables into an ordination analysis for species composition at each site to determine which landscape scale correlated most strongly with species composition.

\section{Results}

Subcatchment delineation \& classification

Forty subcatchments were delineated based on topography within and near the three main catchments. Sites were classified into land-use categories based on $\%$ impervious surface cover (ISC) at three scales: subcatchment, $1-\mathrm{km}$ and $500-\mathrm{m}$ radius site buffers (Table 2-1). Twelve subcatchments were classified as urban, with one in Goose Creek watershed and eleven in Beargrass Creek watershed. Rural subcatchments included eleven in Harrods and three in Goose Creek. Suburban subcatchments spanned all three catchments, with eight in Goose and three each in Beargrass and Harrods Creek watersheds (Table 2-1). Impervious surfaces within 500-m and 1-km of each site generally decreased compared with values at the subcatchment scale. Therefore, as the scale of the buffer distance around each site decreased, the number of sites classified as urban fell, while sites classified as suburban and rural increased (Table 2-1). One site in Beargrass Creek watershed on a privately owned farm was reclassified as rural when analyzed using the 500-m radius site buffer. The site was kept primarily in mown grass, with a thin strip of woody vegetation allowed to persist adjacent to the creek. All sites with $\geq 30 \%$ ISC within $1-\mathrm{km}$ and $500-\mathrm{m}$ radii of a site were located in Beargrass Creek watershed. 
Riparian bank height and zone width variation

Bank height at each site ranged from $15-$ to $230-\mathrm{cm}$. Average bank height was found to vary among the three land uses $(p=0.08$; Fig. 2-4). Pairwise comparisons revealed urban streambanks $($ mean $=122-\mathrm{cm})$ were higher than suburban streambanks (mean $=86-\mathrm{cm} ; \mathrm{p}=0.068)$. Mean rural streambank height was 96-cm. Bank height followed a general upstream-to-downstream pattern of lower-to-higher banks among all land-use categories, with banks getting higher as upstream cumulative catchment area increased (data not shown).

Riparian width was measured at each site to determine the number of plots to establish per site. Suburban sites exhibited the smallest overall width, while rural sites were widest, thus permitting the greatest number of multi-plot sites (Table 2-2). Six of fourteen rural sites, four of fifteen suburban and three of twelve urban sites were less than 12 meters wide, resulting in smaller plot sizes in these sites. Only two urban sites contained more than one plot. One of the sites, located in a city park (Cherokee Park), contained two plots. The other site accomodated three plots, since it extended approximately $100-\mathrm{m}$ before meeting a highway. All suburban sites contained only 1 plot as all vegetated riparian sites were less than 30 meters wide.

The distribution of riparian widths at urban and rural multi-plot sites was similar (Table 2-2). In rural subcatchments, two of the multi-plot sites were along $1^{\text {st }}$ order reaches, one occurred along a $2^{\text {nd }}$ order reach, and two lay adjacent to $3^{\text {rd }}$ order streams. One of the rural multi-plot sites along a $1^{\text {st }}$ order reach appeared to have an altered stream flowpath, as it was located along a residential street and directly abutted an adjacent 
upland slope. The two multi-plot sites in the urban subcatchments occurred along $2^{\text {nd }}$ and $3^{\text {rd }}$ order reaches.

The distribution of narrow riparian zones with respect to stream order showed more variation between land-use categories (Table 2-2). Seven of the sites with riparian widths less than 12 meters were found along first and second order streams. In suburban subcatchments, three of the smaller plots were located along $1^{\text {st }}$ order streams, and one was located along a $3^{\text {rd }}$ order stream. Two of the urban riparian sites with less than $12-$ meter riparian widths were located along $1^{\text {st }}$ order streams, and one along a $3^{\text {rd }}$ order

stream. Both of the narrow sites along $3^{\text {rd }}$ order streams (one along urban and one along a suburban stream) appeared to have been dramatically altered, possibly to contain floodwaters. In the urban area, the first terrace out of the stream channel contained a narrow riparian zone $(<12-\mathrm{m}$ wide $)$ which ended at a vertical incline leading to a second terrace approximately 6 feet above the first terrace. The opposite bank was practically identical.

\section{Plant Communities In Relation To Land-cover}

The distribution of vegetative cover was uneven among the three land-use categories. Three rural sites and four rural nearstream plots had no trees. Two suburban sites had no trees, and all suburban sites were only wide enough for one plot. All urban sites and nearstream plots contained trees. Four rural, six suburban and four urban sites contained no saplings. When only nearstream plots were considered, the pattern remained the same. Two rural, two suburban and three urban sites contained no tree seedlings. The 
distribution of tree seedlings was again the same for nearstream plots. Shrubs were absent from three suburban sites, but all other sites contained shrubs.

Urban, suburban, and rural woody species composition showed distinct patterns, but with common themes across categories. Each land-use category contained unique dominant woody species (Table 2-3). In addition, the most dominant species within landuse categories changed among woody vegetation strata, indicating some measure of succession is occurring within the three categories. The most abundant species also tended to be the more ubiquitous species across land-use categories, and woody species that were unique to a particular land-use category occurred in low frequencies (one or two sites; Tables 2-4 to 2-7). Shared species across all land-use categories included Acer negundo and Celtis occidentalis (Table 2-3 and 2-4). Both species had the lowest tree densities in rural riparian areas. $A$. negundo was also present in all woody vegetation strata across land-use categories (Tables 2-3 to 2-6). The most frequently occurring shrub species was the invasive exotic, Lonicera maackii (Table 2-7). It was located in a majority of all sampled sites and occurred in all urban locations. The mean density of $L$. maackii in urban sites was three times greater than in either suburban or rural areas. The only facultative wetland (FACW) shrub species, Lindera benzoin, occurred in all landuse categories, but it occurred most frequently and with the greatest density in rural areas. An obligate wetland (OBL) species, Rosa palustris, occurred in only one site, which was urban.

Tree diameter distributions also showed some patterns across land-use categories. Most trees ranged between 2.5- and 25-cm in diameter at breast height (DBH) (Table 2-8, Fig. 2-5). Rural riparian areas contained nearly half the number of trees as urban or 
suburban areas in the 5-10 and the 10-25-cm diameter categories. Suburban areas contained the fewest trees greater than $35-\mathrm{cm}$ DBH. In urban and rural subcatchments, Platanus occidentalis (FACW) only occurred in the higher diameter classes $(>30-\mathrm{cm}$ $\mathrm{DBH})$ and only in the smaller diameter classes in suburban areas $(5-10-\mathrm{cm} \mathrm{DBH})$. Species richness of woody vegetation can differ dramatically along an urban-torural gradient (Burton et al. 2005, Burton and Samuelson 2008). As such, species richness for woody plant communities was computed as one measure of species diversity and was computed individually for trees, saplings, tree seedlings, and shrubs. As area sampled differed among nearstream plots and among sites due to variation in number of plots per site, species richness was transformed as in Nilsson (1997) (species richness/log(area sampled)). Analysis at the nearstream scale was conducted to control for any differences in species diversity caused by varying riparian widths (site scale) across land-use categories, and also to more fully capture riparian plant diversity across the width of the riparian zone (site scale). Regressions of species richness of the various woody vegetation strata against \%IS at the subcatchment scale revealed no strong trends. However, species richness patterns within limited ranges of impervious surface were evident. To explore these patterns I grouped site and nearstream plots into land-use categories based on proportion of impervious surface at the subcatchment scale. No significant differences in richness were evident among land-use categories for trees $\geq$ 2.54 DBH, saplings, or shrubs (Table 2-9). Seedling richness, however, differed significantly among land-use types at both the nearstream and site scales of analysis (Fig. 2-6, Table 2-9). Tukey's HSD pairwise comparisons revealed suburban sites contained nearly twice the seedling richness (site and nearstream mean $=2.8$ species) of urban sites 
(site mean $=1.5$ species; nearstream mean $=1.3$ species $)$ at both the site and nearstream scales (Table 2-10). Acer negundo and Celtis occidentalis were the most frequently occurring tree seedling species in urban ( $42 \%$ and $33 \%$ site occurrence, respectively), suburban (60\% site occurrence for both species), and rural (43\% and $36 \%$ site occurrence, respectively) riparian zones. These two species were also the densest in urban riparian zones ( A. negundo -3935 stems/ha; C. occidentalis -1389 stems/ha). C. occidentalis (2556 stem/ha) and Asimina triloba (1444 stems/ha) were the densest in suburban riparian areas. In rural riparian zones, $F$. americana (1647 stems/ha) and $A$. negundo (1210 stems/ha) were the densest (Table 2-6).

Additional structural and community characteristics for woody species used for exploring relationships with land-cover attributes were stem density, tree diameter, and diversity indices (Shannon's, Simpson's reciprocal, Shannon's equitability). As with species richness, I used regression analysis to explore relationships with \%IS at the subcatchment scale. Again, no strong correlations were found, so I grouped nearstream plots into the three land-use categories (urban, suburban, and rural) to further explore these relationships. Tree, sapling, shrub, and tree seedling densities were statistically analyzed at both site and nearstream scales within urban, suburban, and rural land-use categories (subcatchment scale). No statistically significant differences between land-use types (mean site-scale densities reported) were revealed in sapling $(\mathrm{U}=521, \mathrm{~S}=2092, \mathrm{R}$ $=577$ stems $/$ ha $)$, tree seedling $(\mathrm{U}=5729, \mathrm{~S}=11879, \mathrm{R}=5595 \mathrm{stems} / \mathrm{ha})$, or shrub densities $(\mathrm{U}=20219, \mathrm{~S}=10276, \mathrm{R}=15920$ stems $/$ ha) even after eliminating differences in plot size by considering only full-size nearstream plots $(n=27$ plots $)$. I tested whether tree density and DBH varied by land-use category at the nearstream and site levels by 
first using all sites and nearstream plots, and second using only treed sites and nearstream plots. Significant differences at the alpha $\leq 0.1$ level were found among tree $(\geq 2.54-\mathrm{cm}$ DBH) densities between land-use categories at the nearstream scale when all nearstream plots were used. Mean tree density ( $477 \mathrm{stems} / \mathrm{ha})$ in rural plots was about half that in urban plots (mean $=954$ stems/ha, $p=0.073$; Fig. 2-7), with suburban plots being intermediate ( 705 stems/ha). When only treed plots were used no statistically significant differences were evident in tree densities among land-use categories. All urban nearstream plots contained trees $\geq 2.54 \mathrm{~cm} \mathrm{DBH}$. Two non-treed nearstream plots occurred in rural subcatchments (one was a $100-\mathrm{m}^{2}$ full size plot and the other was 50$\mathrm{m}^{2}$ ). Four non-treed nearstream plots occurred in suburban subcatchments (two were in $100-\mathrm{m}^{2}$ full size plots and two were in plots smaller than $100-\mathrm{m}^{2}$ ). All woody species (trees, saplings, seedlings, and shrubs) from nearstream plots were used to compute Shannon's diversity and Simpson's reciprocal indices, as well as Shannon's equitability index. No significant differences were observed among land-use categories for Shannon's and Simpson's diversity indices. Shannon's equitability index did, however, differ significantly among land-use types $(\mathrm{p}=0.047)$, as urban sites demonstrated lower evenness than suburban sites (mean $=0.54$ and 0.77 , respectively) as determined by Tukey's HSD pairwise comparisons ( $\mathrm{p}=0.037$; Fig. 2-8).

Most of the exotic species found were shrub species (Appendix 4). Scatterplots revealed an increasing proportion of exotic shrub species ( $p=0.0003$, Pearson's $r=0.53$ ) and abundances ( $p=0.0003$, Pearson's $r=0.54)$ with increasing \% ISC (subcatchment scale; Fig. 2-9). Above 10\% ISC, at least half of the shrub species occurring within the sites were exotic (Fig. 2-9a). Above 30\% ISC, at least $40 \%$ of all shrub stems were from 
exotic species, with most sites containing $80 \%$ or greater exotic stem densities (Fig. 2$9 b)$.

To control for differences in vegetation that could be related with stream order, I chose sites along first order streams to explore whether patterns in diversity and abundance could be detected by land use (Appendix 5). The strongest trend was a negative relationship between tree seedling density and \%IS (subcatchment scale; $p=$ 0.07 , Pearson's $r=0.39$ ). A similar negative correlation was observed between tree species richness and bank height $(\mathrm{p}=0.055$, Pearson's $\mathrm{r}=0.39)$; however, this relationship was caused by a single influential point with the greatest proportion of impervious surface.

\section{NMS \& Cluster analysis}

I conducted a cluster analysis to obtain groupings of research sites based on commonalities in species composition. I computed Partana ratios and silhouette widths for two to ten clusters to determine the number of clusters to use (Fig. 2-10). The 2cluster configuration gave the highest Partana ratio, but when applied, all but one of the research sites were placed into a single cluster. Partana ratios were similar for three to ten cluster configurations, but overall silhouette widths differed more dramatically. I decided to use cluster regimes with three and five site groupings, since they exhibited the highest overall silhouette widths ( 0.18 and 0.16 , respectively). The quality of the groupings can be seen in silhouette plots for the 3-cluster (Fig. 2-3) and 5-cluster configuration (Fig. 2-

11). The 3-cluster configuration contained one cluster with 26 sites, a second cluster with 12 and a third cluster with 3 sites. The 5 -cluster configuration resulted in more evenly 
sized clusters (Fig. 2-11). Three of the clusters had fewer than ten sites each, with the largest cluster containing 20 sites. One of the clusters (Cluster-4) contained two sites and had a negative average silhouette width, indicating a poor grouping likely containing outliers.

Clusters were analyzed for differences in species composition using frequency of occurrence and indicator species analysis. Species composition of the 3-cluster configuration was relatively unique for each cluster (Table 2-11). The dominant species in cluster-1 was Symphoricarpos orbiculata, a native shrub. Cluster-2 was dominated by Lindera benzoin (native shrub) and Asimina triloba (native tree). Major species in cluster-3 were Lonicera maackii (exotic, invasive shrub) and Celtis occidentalis (native tree). Those species with significant frequencies of occurrence in each of the clusters $(\mathrm{p} \leq$ 0.05 ), determined using indicator species analysis, are shown in Tables 7 and 8 . The shrub layer was found to be important in defining groupings, as each cluster contained a significant indicator shrub species, but not necessarily a tree, sapling, or tree seedling species.

The 5-cluster configuration showed similar species assemblages, but with more overlapping species assignments (Table 2-12). The L. benzoin/A. triloba cluster remained intact as cluster-1, with other notable species including Aesculus glabra and Ulmus rubra (native trees). Cluster-3 was the $L$. maackii-dominated cluster, also containing the nearly ubiquitous species, $A$. negundo and $C$. occidentalis. This was also the largest cluster in the configuration, containing 20 sites. Clusters -2 and -5 shared a common dominant species, $A$. negundo, although the form of the species differed among the groups, with trees dominating cluster-2 and seedlings dominating cluster-5. Both cluster-2 and cluster- 
5 shared species with cluster-1, with $L$. benzoin in cluster- 2 and the less frequent Ligustrum sinense (exotic shrub) in cluster-5.

Non-metric multidimensional scaling (NMS) was used to determine cluster associations with environmental variables and species attributes (e.g., native status, wetland indicator status). NMS was run using the 41 sites organized into a distance matrix using Sorensen's index based on woody species Importance Values (IV's). A three-dimensional configuration was chosen, achieving a final stress of 17.7 and instability of 0.00048 for the final configuration. No site overlap occurred in 3 -cluster or 5-cluster groupings in three dimensions, indicating satisfactory agreement between the cluster analysis and the NMS solution (Fig. 2-12 \& 2-13).

Clusters were analyzed in relation to environmental variables that might correlate with and potentially help explain the observed variation in species composition among clusters (Table 2-13). Environmental variables calculated for each site were entered into a site-by-variable matrix and included as a secondary matrix in NMS using PC-Ord. The biplot feature in PC-Ord was used to generate a line for each variable with an $\mathrm{r}^{2} \geq 0.15$ for one or more axes, with the direction of the line corresponding to the direction of positive correlation of the variable, and the length of the line indicating the strength of the correlation along one or more axes. Implied in the ordination plot but not visible, a vector of equal length but opposing sign points in the opposite direction to indicate a negative correlation.

Percent impervious surface and NLCD categories were included in the NMS as environmental variables (Fig. 2-14a-b) to evaluate cluster identity correlations with coarse and fine scale land-cover variables (Table 2-13; Fig. 2-14b). Cluster-3, having the 
exotic shrub Lonicera maackii as its indicator species in the 3-cluster configuration, was the most "urban" of the clusters, being most strongly correlated with \% IS within a 500-m radius of each plot, as indicated by the length of this vector (Fig. 2-14a). At this smallest scale, low-intensity developed land most strongly correlated with this cluster (Fig. 214b). This type of land-cover is typically composed of single-family residential housing, and was the most frequently observed impervious land-cover type found in this study. To a lesser extent, medium-intensity and open-space developed land also positively correlated with the species composition of cluster-3, followed by high-intensity developed land, which occurred around a few of the most urban sites. Cluster-2, having the native shrub Lindera benzoin as its indicator species, was most positively correlated with the proportion of deciduous forest cover. Cluster-1 with another native shrub Symphoricarpos orbiculata as its indicator species, was most visibly associated with the proportion of pasture covering the land within $500-\mathrm{m}$, with two of the sites containing the greatest proportion of pasture than any of the other sites (not shown).

The NMS configuration was the same for the 5-cluster assignment even as site cluster identities changed the perceived layout. Cluster-3 was the most "urban" cluster in the 5-cluster configuration (Fig. 2-15a). Clusters -1 and -2 were the "deciduous forest" clusters, and cluster- 5 contained the two sites with the highest proportion of pasture (Fig. $2-15 b$.). Figure $2-12$ also demonstrates that $\%$ ISC at the $500-\mathrm{m}$ buffer most strongly explained the variation among clusters. Clusters- 1 and -2 contained $15 \%$ or less ISC within $500-\mathrm{m}$ of each site. Cluster-3 communities were surrounded by $15 \%$ or more ISC at each site. Sites in Cluster-5 were intermediately placed in ordination space and were associated with between 10 and $20 \%$ ISC within a 500-m radius. Association of $\%$ ISC 
with Cluster-4 was not considered, as it was thought to be a poor cluster with two outlier sites.

Unique indicator species among the deciduous forest and urban clusters in the 3cluster and 5-cluster configurations include the native species, L. benzoin and A. triloba, in the deciduous forest clusters (Cluster-2 and Cluster-1, respectively) and the exotic shrub, L. maackii, in the urban clusters (Cluster-3 in both configurations; Tables $7 \& 8$ ). The tree species, C. occidentalis, also occurred most frequently in the urban clusters. $L$. maackii is an exotic invasive shrub growing ubiquitously across the landscape in both riparian and upland habitats. It is considered a naturalized species as it successfully propagates itself independently of human interference. The other three species are native to the area. L. benzoin is also a facultative wetland species, occurring most frequently in wetland habitats (Appendices $2 \& 4$ ).

Wetland indicator and native status for each species (Appendix 2) was included in the analyses as environmental variables to assess which categories were associated with the same site clusters as the land-cover variables. In this way an indirect correlation could be drawn between these species attributes and land-cover variables. Figure $2-16 a-b$ shows the direction of correlation of these attributes in relation to the various site clusters. Facultative wetland (FACW) and native species attributes most strongly correlated with the deciduous forest clusters (Clusters-2 and -1 in the 3- and 5-cluster configuration). Facultative upland species did not positively associate with any particular grouping of sites. L. maackil, while contributing to the arrangement of sites in the NMS configuration, was not included in the calculation of species attribute values due to its overwhelming dominance in the urban sites, which would have masked any associations 
of urban clusters with species attributes of other species. Had it been included, the urban groupings (Cluster-3) would have shown a strong correlation with exotic species as it was the sole significant indicator species of those groupings (Table 2-11 \& 2-12).

Since L. maackii was such an important species in urban clusters, I decided to compare its relative importance within sites with proportion of impervious surface at the subcatchment scale and within 500-m of the sites. L. maackii IVs significantly increased with increasing proportions of impervious surface within $500-\mathrm{m}$ of site centers $(\mathrm{p}=$ $0.000057, \mathrm{r}^{2}=0.34 ;$ Fig. 2-17). In addition, tree seedling densities and, to a lesser extent, tree sapling densities showed a markedly decreasing trend with increasing L. maackii site densities (Fig. 2-18). The highest seedling and sapling densities occur at the lowest densities ( $<5000$ to $10000 \mathrm{stems} / \mathrm{ha}$ ) of L. maackii, and the lowest seedling and sapling densities at high $L$. maackii stem densities $>10000$ stems/ha. These results show $L$. maackii to be an urban species with potentially negative impacts on the regeneration layer for other woody plants.

\section{Discussion}

This research revealed that some components of riparian woody species structure and composition, as well as density and diversity, correlated with land-cover metrics associated with urban land use. Distinct plant assemblages were found based partially on an urbanization gradient. The 3-cluster configuration (Fig. 2-12) highlights the primary species differences between the riparian sites (Table 2-11), essentially splitting them into "deciduous forest" (cluster-2) and "urban" (cluster-3) with a small "pastoral" cluster (cluster-1). All three clusters were distinguished by different indicator shrub species. $S$. 
orbiculata, L. benzoin, and L. maackii, which respectively defined clusters $-1,-2$, and -3 (Table 2-11) and suggests that those species have differing tolerances for disturbance, such as flooding frequency and intensity, or other anthropogenically altered landscape level factors. The tree, A. triloba, was also an indicator species for cluster-2, and is listed together with $L$. benzoin as a riparian species by Samuelson and Hogan (2006). Cluster 2 being the least-urban, most-forested cluster (Fig. 2-14) indicates those particular species are likely sensitive to factors such as disturbance associated with pastured (free-roaming) livestock and those associated with urban development within a 500-m radius. L. benzoin was the most prominent facultative wetland species in this study, occurring almost exclusively in rural riparian habitats (Fig. 2-16, Table 2-11 \& 2-12) at all scales. $L$. maackii has been positively correlated to \% IS and urban areas in other studies (Luken and Thieret 1996, Hutchinson and Vankat 1997, Borgmann and Rodewald 2005). For example, Borgmann (2005) also found a positive relationship between the degree of urban land cover within 1-km of riparian areas and percent cover of L. maackii. Exotic species invasions in urban riparian zones has been correlated with greater natural (flooding) and anthropogenic (trampling) disturbances (Moffatt and McLachlan 2004, Burton et al. 2005, Burton and Samuelson 2008). These findings indicate that as areas become more urbanized, exotic shrubs, such as L. maackil, will assume greater occurrences and densities within riparian zones.

More discrete plant communities were found when the 5-cluster configuration (Fig. 2-13) was used for grouping communities than when the 3-cluster grouping was used. The "deciduous forest" cluster from the 3-cluster configuration was separated into two distinct clusters (Fig. 2-15). Cluster-1, containing both L. benzoin and A. triloba was 
most similar to the original three-cluster assemblage. Cluster-2 also contained L. benzoin, with $A$. negundo assuming the greatest importance within the assemblage (Table 2-12). The relative absence of $A$. triloba from this cluster could indicate environmental factors non-conducive to the retention, establishment and growth of this species over the more ubiquitous A. negundo (Samuelson and Hogan 2006), which occurred within all clusters. The degree of sensitivity of $A$. triloba and $L$. benzoin to \% ISC is shown in Figure 2-13. Cluster-1, containing both species, ends at $10 \%$ ISC when calculated within a $500-\mathrm{m}$ radius of a site. Cluster- 2 communities are found when ISC increases to $15 \%$, suggesting that $A$. triloba is more tolerant to increasing urban conditions than L. benzoin.

Some of the urban factors that might affect riparian plant assemblages include human manipulation of riparian areas (mowing, selective cutting of brush), human choices for plantings and manipulation of land close to naturalized riparian areas (yard plantings, pesticide and fertilizer use, pets), urban wildlife effects (deer and small mammal herbivory and seed predation and dispersal), habitat fragmentation, edge effects, the urban heat island, air pollution and urban hydrological drought. In this study, I attempted to explore the possible effect of urban hydrologic drought (Groffman et al. 2003) on riparian species composition, since it has been implicated as a possible determinant of riparian species composition (Tickner et al. 2001, Burton et al. 2005). I used bank height as an indicator of this phenomenon, where the highest banks would indicate the possibility of urban hydrologic drought. I found no direct relationship between species composition or diversity and bank height, despite the fact that sites classified as urban at the subcatchment level were found to contain significantly higher streambanks than suburban areas. However, depth to groundwater does not necessarily 
equate to soil dryness in the upper soil horizons where seedlings and shallow rooted species would be most affected. Other factors that may contribute to soil dryness include soil type and compaction, the urban heat island effect, proportion of impervious surface and frequency of storm drains, stream flooding frequency, riparian width and proportion covered by vegetation. The rarity of wetland species in urban riparian zones does indicate that soil dryness may contribute to their loss of dominance, but the absence of a correlation with bank height suggests that other factors may also explain their rarity. With respect to determining the potential effects of depth to groundwater as a control on riparian plant community composition, additional research should be focused on more directly eliciting the effects that soil dryness and interactions with other soil characteristics in upper horizons have on species composition via manipulated experiments.

A new conceptual model is presented in Fig. 2-19 to reflect the findings of this study and offer a potential area of focus for future studies. In these watersheds, depth to groundwater (as measured by bank height) does not appear to provide major selective pressures on species establishment and growth compared to some other factors. Degree of soil moisture as determined by precipitation patterns, as well as other environmental conditions (light penetration, proportion of watershed that is forested) may be stronger determinants of the species that successfully establish in urban and suburban environments.

Tree seedling richness was significantly lower in urban areas than in suburban areas (Table 2-8, Fig. 2-6), but tree seedling densities did not differ. Equally high seedling density indicates that the lower richness in urban areas is not due to fewer 
individuals surviving current conditions, and instead suggests that a) the supply of propagules to urban areas is less diverse than in suburban areas, $b$ ) urban conditions only allow a very limited number of species to propagate, and/or c) biotic factors like selective herbivory, are affecting successful species establishment.

Both sapling and seedling densities exhibited a decreasing trend with greater $L$. maackii densities (Fig. 2-18a-b). Reductions in seedling and sapling densities under high proportions of exotic shrubs have been shown in other studies (Merriam and Feil 2002, Loewenstein and Loewenstein 2005, Burton and Samuelson 2008). Exotic invasive shrubs pose a growing threat to overstory species regeneration. Rural tree densities were also significantly lower than that in the other two land-use types determined at the subcatchment scale. Large tree diameters have been shown to contribute to greater spacing between trees (Porter et al. 2001), yet I found no significant differences existed in mean tree DBH between land-use types at the subcatchment scale. Differences in tree densities were attributed to the absence of trees at several rural and suburban sites, since elimination of those plots without trees resulted in no significant differences between land-use types in tree density. Plot size was not indicated as a major contributing factor to these differences in tree densities among land uses either, since a majority of the smaller plots for all sites contained trees, and half of the non-treed nearstream plots were full size $\left(100-m^{2}\right)$.

Woody plant diversity as measured by species richness, Shannon's and Simpson's diversity indices, and Shannon's evenness index did not significantly differ among landuse categories in this study. This trend was in contrast to that found by Burton et al. (2005) in the Atlanta, Georgia area, where Shannon diversity for woody species in 
riparian zones increased with distance from the city center. In my study, woody plant community evenness was lower in urban areas than in suburban areas, indicating that, if these communities are not directly managed, natural succession in urban sites favors success of a few exotic shrubs. Restoration managers attempting to reclaim an urban riparian area should be selective when choosing species to include along streams. Also, some form of management plan may need to be formed to control for specific exotic plants, such as L. maackii, that limit understory growth.

Exotic, naturalized vegetation was more highly associated with the urban riparian communities due to the high prevalence of L. maackii. This shrub occurred in all land-use categories, but dominated urban sites, correlating most strongly with proportion of impervious surface within a $500 \mathrm{~m}$ site radius (Fig. 2-17). Brown and Peet (2003) showed higher species richness of both native and exotic species with flooding frequency in mountainous riparian areas. This was attributed to immigration-driven selection pressures due to propagule deposition and frequent disturbance. Similar findings were reported by Burton et al. (2005) and Burton and Samuelson (2008). These same pressures may be at work in urban riparian areas, coupled with conditions unique to urban environments (heat island, potentially drier soils, more edge habitat) that allow exotic generalist species to gain a foothold over native riparian species once they arrive.

Two of the more ubiquitous tree species across land-use types were $A$. negundo and $C$. occidentalis. Both species are tolerant of a wide range of soil and moisture conditions. A. negundo is the most widely distributed species within its genus in North America, extending from coast to coast and from Canada south to Texas and Florida. $C$. occidentalis is widely distributed within the eastern continental U.S., although it does not 
extend as far north as $A$. negundo. Both species are equally drought tolerant, and able to withstand prolonged periods of inundation exceeding 100 days (Burns and Honkala 1990).

The findings regarding riparian widths have implications for restoration efforts. Width of the riparian zone has been shown to be important for maintaining amphibian and bird species (Rudolph and Dickson 1990, Kinley and Newhouse 1997) and for the ability of the riparian area to buffer undesirable nutrient inputs into streams from adjacent land. Reestablishing riparian zones with appropriate widths will require reverse engineering to counter the efforts that created the narrow riparian widths along high order streams in urban and suburban locations. This, along with hydrological characteristics of riparian zones as they relate to species composition and ecosystem services, should continue to be studied to determine species responses to development and the resulting alteration to riparian functionality.

\section{Conclusions}

Woody plant assemblages are influenced by the proportion of impervious surface within a 500-m to $1-\mathrm{km}$ radius. Exotic shrubs, such as $L$. maackii, showed a high affinity for urban sites. Wetland species exhibited a low affinity for these urban sites, and instead occurred in those sites with a greater proportion of deciduous forest.

In addition to influencing species composition, the various growth stages of woody species displayed different paterns along this urban-to-rural gradient of riparian sites. Tree seedling richness declined in urban compared to suburban riparian areas. However, tree seedling densities did not differ among sites within these two land-use 
categories. Tree, sapling, and shrub species richness did not significantly differ among sites across the urban-to-rural land use gradient. However, tree densities were significantly lower in rural riparian zones than in suburban or urban sites due to the higher number of plots with no trees in rural areas.

I expected certain environmental conditions, such as bank height, within the associated riparian area to influence plant species assemblages and diversity. While mean bank height was highest adjacent to urban riparian zones, it did not explain the variation in woody species composition across sites. Instead, the high prevalence of $L$. maackii in sites with relatively high proportions of impervious surface surrounding them appeared to exhibit the greatest associations with plant abundances. Sapling and tree seedling densities both declined with increasing densities of this exotic, invasive shrub.

Land managers and riparian restoration teams will need to consider these findings when considering woody species to plant in riparian areas. For example, woody species survivorship in different land-use contexts will vary, making the choice of species for introducing or re-introducing into the riparian zone more risky from a resource perspective. 
Table 2-1: Area of research catchments and the number of study sites classified as urban (U), suburban (S) and rural (R) at the subcatchment (SC) scale, and within a 1-km and $500-\mathrm{m}$ radius from each study site. Classification as urban (U), suburban (S) and rural (R) land cover was based on proportion of impervious surface with $\geq 30 \%$ being urban, $\leq 10 \%$ being rural, and between 10 and $30 \%$ being suburban.

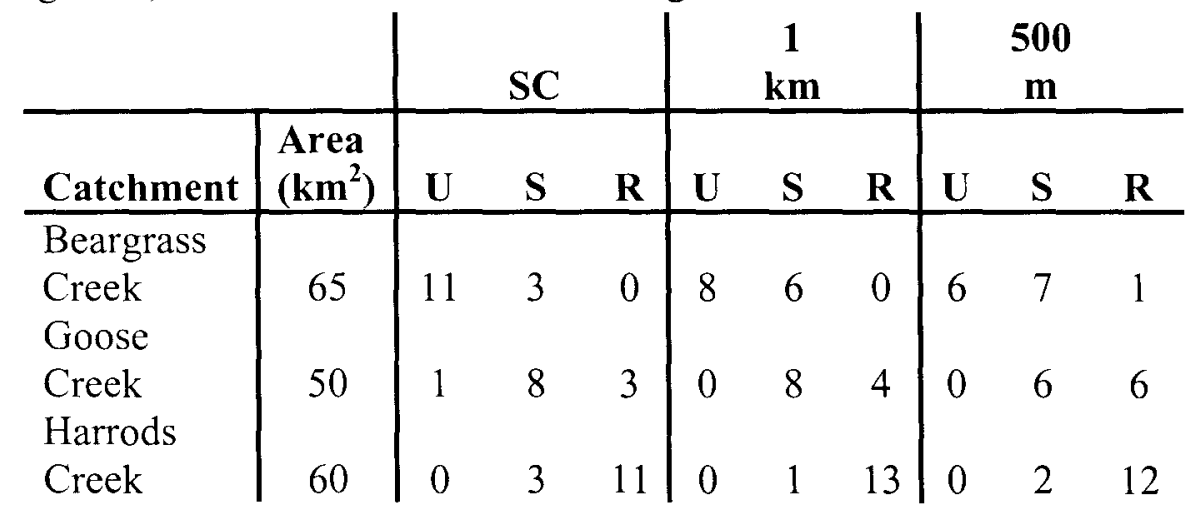


Table 2-2: Distribution of riparian width intervals by stream order and land-use category at the subcatchment scale. Riparian zone width was measured along areas of level ground beginning at the streambank edge and extended until reaching a structural barrier (such as a road, parking lot, or building), upland slope, or other obstruction (grazed pasture, ephemeral stream channel). Classification as urban, suburban and rural land cover was based on proportion of impervious surface with $\geq 30 \%$ being urban, $\leq 10 \%$ being rural, and between 10 and $30 \%$ being suburban.

\begin{tabular}{lccccc} 
& & \multicolumn{5}{c}{$\begin{array}{c}\text { Riparian } \\
\text { width (m) }\end{array}$} \\
Land use & $\begin{array}{c}\text { Stream } \\
\text { Order }\end{array}$ & $<\mathbf{1 2}$ & $\mathbf{1 2 - 4 5}$ & $\mathbf{4 6 - 8 5}$ & $\mathbf{> 8 5}$ \\
\hline Urban & $\mathbf{1}$ & 2 & 4 & 0 & 0 \\
& $\mathbf{2}$ & 0 & 1 & 0 & 1 \\
& $\mathbf{3}$ & 1 & 2 & 1 & 0 \\
\hline Total & & 3 & 7 & 1 & 1 \\
\hline Suburban & $\mathbf{1}$ & 3 & 7 & 0 & 0 \\
& $\mathbf{2}$ & 0 & 4 & 0 & 0 \\
Total & $\mathbf{3}$ & 1 & 0 & 0 & 0 \\
\hline Rural & $\mathbf{1}$ & 4 & 11 & 0 & 0 \\
& $\mathbf{2}$ & 2 & 2 & 2 & 0 \\
& $\mathbf{3}$ & 0 & 1 & 1 & 0 \\
Total & & 6 & 3 & 5 & 0
\end{tabular}


Table 2-3: Relative importance value of dominant ( $>5 \%$ ) woody species by strata within land-use categories at the subcatchment scale.

\begin{tabular}{|c|c|c|c|}
\hline Land Use1 & Strata & Species & $\begin{array}{c}\text { Relative } \\
\text { IV }\end{array}$ \\
\hline \multirow{24}{*}{ Rural } & \multirow{5}{*}{ Seedling } & Fraxinus americana $L$. & 36 \\
\hline & & Acer negundo $L$. & 28 \\
\hline & & Acer saccharum Marsh. & 25 \\
\hline & & Celtis occidentalis $L$. & 23 \\
\hline & & Asimina triloba (L.) Dunal & 16 \\
\hline & \multirow{7}{*}{ Saplings } & Asimina triloba (L.) Dunal & 54 \\
\hline & & Robinia pseudoacacia L. & 35 \\
\hline & & Celtis occidentalis L. & 25 \\
\hline & & Acer negundo $L$. & 22 \\
\hline & & Aesculus glabra Willd. & 20 \\
\hline & & Ulmus rubra Muhl. & 20 \\
\hline & & Fraxinus americana $L$. & 16 \\
\hline & \multirow{7}{*}{ Trees } & Juniperus virginiana $L$. & 46 \\
\hline & & Juglans nigra $L$. & 33 \\
\hline & & Maclura pomifera (Raf.) Schneid. & 19 \\
\hline & & Acer saccharum Marsh. & 16 \\
\hline & & Acer negundo L. & 15 \\
\hline & & Asimina triloba (L.) Dunal & 14 \\
\hline & & Fraxinus americana $L$ & 13 \\
\hline & \multirow{5}{*}{ Shrubs } & Symphoricarpos orbiculatus Moench & 58 \\
\hline & & Lonicera maackii (Rupr.) Herder* & 45 \\
\hline & & Lindera benzoin (L.) Blume & 32 \\
\hline & & Ligustrum sinense Lour. ${ }^{*}$ & 25 \\
\hline & & Rubus spp. & 22 \\
\hline \multirow{11}{*}{ Suburban } & \multirow{7}{*}{ Seedling } & Fraxinus pennsylvanica Marsh. & 30 \\
\hline & & Celtis occidentalis L. & 27 \\
\hline & & Acer negundo L. & 24 \\
\hline & & Asimina triloba (L.) Dunal & 18 \\
\hline & & Acer saccharum Marsh. & 14 \\
\hline & & Prunus serotina Ehrh. & 12 \\
\hline & & Ulmus rubra Muhl. & 12 \\
\hline & \multirow{4}{*}{ Saplings } & Staphylea trifolia $L$ & 49 \\
\hline & & Asimina triloba (L.) Dunal & 26 \\
\hline & & Acer negundo $L$. & 14 \\
\hline & & Fraxinus pennsylvanica Marsh. & 13 \\
\hline
\end{tabular}




\begin{tabular}{|c|c|c|c|}
\hline \multirow{9}{*}{$\begin{array}{l}\text { Table } 2-3 \\
\text { (continued) }\end{array}$} & & Fraxinus americana $L$. & 13 \\
\hline & & Celtis occidentalis L. & 13 \\
\hline & & Cornus spp. & 12 \\
\hline & & Ulmus rubra Muhl. & 11 \\
\hline & & Acer negundo $L$. & 46 \\
\hline & & Fraxinus americana $L$. & 21 \\
\hline & & Acer saccharum Marsh. & 20 \\
\hline & & Ulmus rubra Muhl. & 19 \\
\hline & Trees & Staphylea trifolia $L$. & 17 \\
\hline \multirow[t]{18}{*}{ Suburban } & & Acer saccharinum $L$. & 16 \\
\hline & & Cornus alternifolia L. $f$. & 14 \\
\hline & & Tilia americana $L$. & 13 \\
\hline & & Morus alba $L .{ }^{*}$ & 11 \\
\hline & & Lonicera maackii (Rupr.) Herder* & 56 \\
\hline & & Ligustrum sinense Lour. ${ }^{*}$ & 49 \\
\hline & Shrubs & Lindera benzoin (L.) Blume & 26 \\
\hline & & Euonymus alatus (Thunb.) Sieb. * & 23 \\
\hline & & Rubus spp. & 18 \\
\hline & & Acer negundo $L$. & 72 \\
\hline & & Celtis occidentalis $L$. & 41 \\
\hline & Seedling & Fraxinus americana $L$. & 21 \\
\hline & 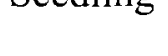 & Aesculus glabra Willd. & 13 \\
\hline & & Gleditsia tricanthos $L$. & 13 \\
\hline & & Morus rubra L. & 13 \\
\hline & & Celtis occidentalis $L$. & 57 \\
\hline & & Acer saccharum Marsh. & 27 \\
\hline & & Acer negundo $L$. & 26 \\
\hline \multirow[t]{9}{*}{ Urban } & Saplings & Morus rubra L. & 15 \\
\hline & & Fraxinus quadrangulata Michx. & 15 \\
\hline & & Fraxinus americana $L$. & 13 \\
\hline & & Fraxinus pennsylvanica Marsh. & 12 \\
\hline & & Platanus occidentalis $L$. & 37 \\
\hline & & Morus alba L.* & 35 \\
\hline & & Acer negundo $L$. & 29 \\
\hline & Trees & Celtis occidentalis $L$. & 27 \\
\hline & & $\begin{array}{l}\text { Fraxinus pennsylvanica Marsh. } \\
\text { Carya cordiformis (Wangenh.) K. }\end{array}$ & 12 \\
\hline
\end{tabular}


Table 2-3

(continued)

Urban
Lonicera maackii (Rupr.) Herder*

Shrubs

Cornus spp.

29

Hibiscus syriacus L.*

25

Lindera benzoin (L.) Blume

Lagerstroemia indica $L .{ }^{*}$

Ligustrum sinense Lour. *
18

17

16

1 Land use category based upon \% impervious surface at the subcatchment scale.

Urban $\geq 30 \%$, Rural $\leq 10 \%$, Suburban between 10 and $30 \%$ IS

$*$ Non-native species

Relative IV calculation is the sum of relative density and relative frequency for seedlings, saplings, and shrubs. Tree relative IV is the sum of relative density and relative basal area. Maximum value is 200 . Only species with IV $>10(5 \%)$ are shown. 
Table 2-4: Tree frequency, mean density and basal area ( \pm S.E.) in land use categories at the subcatchment scale.

\begin{tabular}{|c|c|c|c|c|c|c|c|c|c|c|c|c|}
\hline \multirow[b]{2}{*}{ Species } & \multicolumn{4}{|c|}{ Urban } & \multicolumn{4}{|c|}{ Suburban } & \multicolumn{4}{|c|}{ Rural } \\
\hline & Freq & $\begin{array}{c}\text { Mean } \\
\text { Density } \\
\text { (stems/ } \\
\text { ha) }\end{array}$ & $\begin{array}{c}\text { Mean } \\
\text { Basal } \\
\text { Area } \\
\left(\mathrm{m}^{2} / \mathrm{ha}\right)\end{array}$ & $\begin{array}{c}\text { Mean } \\
\text { Basal } \\
\text { Area }_{2} \\
\left(\mathbf{m}^{2} / \mathbf{h a}\right)\end{array}$ & Freq & $\begin{array}{c}\text { Mean } \\
\text { Density } \\
\text { (stems/ } \\
\text { ha) }\end{array}$ & $\begin{array}{c}\text { Mean } \\
\text { Basal } \\
\text { Area } \\
\left(\mathrm{m}^{2} / \mathbf{h a}\right)\end{array}$ & $\begin{array}{c}\text { Mean } \\
\text { Basal } \\
\text { Area }_{2} \\
\left(\mathbf{m}^{2} / \mathbf{h a}\right)\end{array}$ & Freq & $\begin{array}{c}\text { Mean } \\
\text { Density } \\
\text { (stems/ } \\
\text { ha) }\end{array}$ & $\begin{array}{c}\text { Mean } \\
\text { Basal } \\
\text { Area } \\
\left(\mathbf{m}^{2} / \mathbf{h a}\right)\end{array}$ & $\begin{array}{c}\text { Mean } \\
\text { Basal } \\
\text { Area }_{2} \\
\left(\mathbf{m}^{2} / \mathbf{h a}\right)\end{array}$ \\
\hline $\begin{array}{l}\text { Acer negundo } \\
\text { (FAC) }\end{array}$ & 0.33 & $192(97)$ & $5(3)$ & $16(8)$ & 0.53 & $196(75)$ & $4(2)$ & $8(2.6)$ & 0.43 & $68(29)$ & $1.5(0.8)$ & $3.6(1.6)$ \\
\hline $\begin{array}{l}\text { Acer } \\
\text { saccharum } \\
\text { (FACU) }\end{array}$ & 0.17 & $42(34)$ & $0.72(0.66)$ & $4.3(3.6)$ & 0.27 & $86(39)$ & $1.4(0.8)$ & $5(2)$ & 0.21 & $42(26)$ & $1.6(1.3)$ & $7.3(5.4)$ \\
\hline $\begin{array}{l}\text { Aesculus } \\
\text { glabra } \\
\text { (FACU) }\end{array}$ & 0.08 & $8(8)$ & $0.55(0.55)$ & 6.7 & 0.07 & $7(7)$ & $0.01(0.01)$ & 0.2 & 0.14 & $29(22)$ & $0.1(0.08)$ & $0.8(0.2)$ \\
\hline $\begin{array}{l}\text { Carya } \\
\text { cordiformis } \\
\text { (FACU) }\end{array}$ & 0.08 & $25(25)$ & $2(2)$ & 17.5 & 0 & 0 & 0 & 0 & 0 & 0 & 0 & 0 \\
\hline $\begin{array}{l}\text { Celtis } \\
\text { occidentalis } \\
\text { (FACU) }\end{array}$ & 0.25 & $108(58)$ & $6(3)$ & $22(6)$ & 0.33 & $61(29)$ & $0.09(0.06)$ & $0.3(0.1)$ & 0.14 & $14(10)$ & $0.1(0.1)$ & $1(0.7)$ \\
\hline $\begin{array}{l}\text { Fraxinus } \\
\text { americana } \\
\text { (FACU) }\end{array}$ & 0.17 & $31(21)$ & $1(0.9)$ & $5.8(4.5)$ & 0.20 & $47(27)$ & $1.9(1.3)$ & $9(5)$ & 0.14 & $51(43)$ & $0.7(0.7)$ & $4.7(4.4)$ \\
\hline $\begin{array}{l}\text { Fraxinus } \\
\text { pennsylvanica } \\
\text { (FACW) }\end{array}$ & 0.08 & $43(43)$ & $0.1(0.1)$ & 1.5 & 0.07 & $18(18)$ & $0.02(0.02)$ & 0.2 & 0 & 0 & 0 & 0 \\
\hline $\begin{array}{l}\text { Juglans nigra } \\
\text { (FACU) }\end{array}$ & 0.25 & $36(20)$ & $2(1)$ & $7(2)$ & 0.07 & $13(9)$ & $0.1(0.1)$ & 2 & 0.29 & $25(15)$ & $4(2.5)$ & $15(7)$ \\
\hline $\begin{array}{l}\text { Maclura } \\
\text { pomifera } \\
\text { (UPL) }\end{array}$ & 0.08 & $6(6)$ & $0.04(0.04)$ & 0.5 & 0 & 0 & 0 & 0 & 0.14 & $43(31)$ & $1.5(1.3)$ & $7.9(1.4)$ \\
\hline $\begin{array}{l}\text { Morus alba } \\
\text { (UPL) }\end{array}$ & 0.17 & $205(155)$ & $3(2)$ & $16(4)$ & 0.07 & $20(20)$ & $0.54(0.54)$ & 8 & 0 & 0 & 0 & 0 \\
\hline
\end{tabular}


Table 2-4 (continued)

\begin{tabular}{|c|c|c|c|c|c|c|c|c|c|c|c|c|}
\hline \multirow[b]{2}{*}{ Species } & \multicolumn{4}{|c|}{ Urban } & \multicolumn{4}{|c|}{ Suburban } & \multicolumn{4}{|c|}{ Rural } \\
\hline & Freq. & $\begin{array}{c}\begin{array}{c}\text { Mean } \\
\text { Density } \\
\text { (stems/ } \\
\text { ha) }\end{array} \\
\end{array}$ & $\begin{array}{c}\text { Mean Basal } \\
\text { Area } \\
\left(\mathbf{m}^{2} / \mathbf{h a}\right)\end{array}$ & $\begin{array}{c}\text { Mean } \\
\text { Basal } \\
\text { Area }_{2} \\
\left(\mathrm{~m}^{2} / \mathbf{h a}\right)\end{array}$ & Freq. & $\begin{array}{c}\begin{array}{c}\text { Mean } \\
\text { Density } \\
\text { (stems/ } \\
\text { ha) }\end{array} \\
\end{array}$ & $\begin{array}{c}\text { Mean } \\
\text { Basal } \\
\text { Area } \\
\left(\mathrm{m}^{2} / \mathrm{ha}\right) \\
\end{array}$ & $\begin{array}{c}\text { Mean } \\
\text { Basal } \\
\text { Area }_{2} \\
\left(\mathbf{m}^{2} / \mathbf{h a}\right)\end{array}$ & Freq. & $\begin{array}{c}\text { Mean } \\
\text { Density } \\
\text { (stems/ } \\
\text { ha) }\end{array}$ & $\begin{array}{c}\text { Mean Basal } \\
\text { Area } \\
\left(\mathbf{m}^{2} / \mathbf{h a}\right) \\
\end{array}$ & $\begin{array}{c}\text { Mean } \\
\text { Basal } \\
\text { Area } \\
\left(\mathbf{m}^{2} / \mathbf{h a}\right) \\
\end{array}$ \\
\hline $\begin{array}{l}\text { Morus rubra } \\
\text { (FACU) }\end{array}$ & 0.08 & $13(9)$ & $0.3(0.3)$ & 4 & 0 & 0 & 0 & 0 & 0 & 0 & 0 & 0 \\
\hline $\begin{array}{l}\text { Picea } \\
\text { pungens (NI) }\end{array}$ & 0.08 & $8(8)$ & $0.03(0.03)$ & 0.3 & 0 & 0 & 0 & 0 & 0 & 0 & 0 & 0 \\
\hline $\begin{array}{l}\text { Platanus } \\
\text { occidentalis } \\
\text { (FACW) }\end{array}$ & 0.25 & $38(23)$ & $11(6)$ & $43(14)$ & 0.07 & $7(7)$ & $0.03(0.03)$ & 0.4 & 0.07 & $7(7)$ & $1(1)$ & 13 \\
\hline $\begin{array}{l}\text { Prunus } \\
\text { cerasifera } \\
\text { (NI) } \\
\text { Quercus } \\
\text { rubra } \\
\text { (FACU) }\end{array}$ & 0.08 & $21(21)$ & $0.007(0.007)$ & 0.1 & 0 & 0 & 0 & 0 & 0 & 0 & 0 & 0 \\
\hline $\begin{array}{l}\text { Thuja } \\
\text { occidentalis } \\
\text { (FACW) }\end{array}$ & 0.08 & $3(3)$ & $0.002(0.002)$ & 0.02 & 0 & 0 & 0 & 0 & 0 & 0 & 0 & 0 \\
\hline $\begin{array}{l}\text { Ulmus rubra } \\
\text { (FAC) }\end{array}$ & 0.25 & $41(24)$ & $1(1)$ & $5.4(5.2)$ & 0.40 & $118(67)$ & $1.5(1.3)$ & $3.7(3.1)$ & 0.14 & $25(19)$ & $0.07(0.05)$ & $0.5(0.2)$ \\
\hline $\begin{array}{l}\text { Acer } \\
\text { saccharinum } \\
\text { (FACW) }\end{array}$ & 0 & 0 & 0 & 0 & 0.07 & $27(27)$ & $0.8(0.8)$ & 11 & 0 & 0 & 0 & 0 \\
\hline $\begin{array}{l}\text { Asimina } \\
\text { triloba } \\
\text { (FACU) }\end{array}$ & 0 & 0 & 0 & 0 & 0.13 & $20(14)$ & $0.06(0.05)$ & $0.4(0.3)$ & 0.21 & $101(86)$ & $0.1(0.1)$ & $0.6(0.5)$ \\
\hline $\begin{array}{l}\text { Cornus } \\
\text { alternifolia } \\
\text { (NI) }\end{array}$ & 0 & 0 & 0 & 0 & 0.07 & $35(35)$ & $0.2(0.2)$ & 2.8 & 0 & 0 & 0 & 0 \\
\hline
\end{tabular}


Table 2-4 (continued)

\begin{tabular}{|c|c|c|c|c|c|c|c|c|c|c|c|c|}
\hline \multirow{3}{*}{$\begin{array}{l}\text { Species } \\
\text { Staphylea } \\
\text { trifolia }\end{array}$} & \multicolumn{4}{|c|}{ Urban } & \multicolumn{4}{|c|}{ Suburban } & \multicolumn{4}{|c|}{ Rural } \\
\hline & Freq. & $\begin{array}{c}\text { Mean } \\
\text { Density } \\
\text { (stems/ha) }\end{array}$ & $\begin{array}{c}\text { Mean } \\
\text { Basal } \\
\text { Area } \\
\left(\mathrm{m}^{2} / \mathrm{ha}\right) \\
\end{array}$ & $\begin{array}{c}\text { Mean } \\
\text { Basal } \\
\text { Area }_{2} \\
\left(\mathrm{~m}^{2} / \mathrm{ha}\right) \\
\end{array}$ & Freq. & $\begin{array}{c}\text { Mean } \\
\text { Density } \\
\text { (stems/ha) }\end{array}$ & $\begin{array}{c}\text { Mean } \\
\text { Basal } \\
\text { Area } \\
\left(\mathrm{m}^{2} / \mathrm{ha}\right) \\
\end{array}$ & $\begin{array}{c}\text { Mean } \\
\text { Basal } \\
\text { Area }_{2} \\
\left(\mathrm{~m}^{2} / \mathbf{h a}\right) \\
\end{array}$ & Freq. & $\begin{array}{c}\text { Mean } \\
\text { Density } \\
\text { (stems/ha) }\end{array}$ & $\begin{array}{c}\text { Mean } \\
\text { Basal } \\
\text { Area } \\
\left(\mathrm{m}^{2} / \mathrm{ha}\right) \\
\end{array}$ & $\begin{array}{c}\text { Mean } \\
\text { Basal } \\
\text { Area }_{2} \\
\left(\mathrm{~m}^{2} / \mathrm{ha}\right) \\
\end{array}$ \\
\hline & 0 & 0 & 0 & 0 & 0.07 & $48(48)$ & $0.03(0.03)$ & 0.5 & 0 & 0 & 0 & 0 \\
\hline $\begin{array}{l}\text { Tilia } \\
\text { americana } \\
\text { (FACU) } \\
\text { Carpinus }\end{array}$ & 0 & 0 & 0 & 0 & 0.13 & $40(34)$ & $0.7(0.7)$ & $5(5)$ & 0 & 0 & 0 & 0 \\
\hline $\begin{array}{l}\text { caroliniana } \\
\text { (FAC) } \\
\text { Cercis }\end{array}$ & 0 & 0 & 0 & 0 & 0 & 0 & 0 & 0 & 0.07 & $12(12)$ & $0.03(0.03)$ & 0.4 \\
\hline $\begin{array}{l}\text { canadensis } \\
\text { (FACU) } \\
\text { Cornus }\end{array}$ & 0 & 0 & 0 & 0 & 0 & 0 & 0 & 0 & 0.07 & $14(14)$ & $0.1(0.1)$ & 1 \\
\hline $\begin{array}{l}\text { florida } \\
\text { (FACU) }\end{array}$ & 0 & 0 & 0 & 0 & 0 & 0 & 0 & 0 & 0.14 & $22(15)$ & $0.02(0.01)$ & $0.1(0.003)$ \\
\hline $\begin{array}{l}\text { Juniperus } \\
\text { virginiana } \\
\text { (FACU) } \\
\text { Prunus }\end{array}$ & 0 & 0 & 0 & 0 & 0 & 0 & 0 & 0 & 0.07 & $71(71)$ & $3(3)$ & 39 \\
\hline $\begin{array}{l}\text { Serotina } \\
\text { (FACU) } \\
\text { Quercus } \\
\text { alba }\end{array}$ & 0 & 0 & 0 & 0 & 0 & 0 & 0 & 0 & 0.07 & $4(4)$ & $0.01(0.01)$ & 0.2 \\
\hline (FACU) & 0 & 0 & 0 & 0 & 0 & 0 & 0 & 0 & 0.07 & $4(4)$ & $0.2(0.2)$ & 3 \\
\hline SUM & & $825(87)$ & $32(3.4)$ & $153(3.4)$ & & $742(74)$ & $12(1.2)$ & $56.5(1.2)$ & & $533(57)$ & $14(1.5)$ & $98(1.5)$ \\
\hline
\end{tabular}

${ }^{*}$ Wetland indicator status - Frequency of occurrence in wetlands and non-wetlands: OBL $>99 \%$ in wetlands, FACW $67-99 \%$ in wetlands, FAC equally likely to occur in wetlands and non-wetlands, FACU $67-99 \%$ occurrence in non-wetlands, UPL $>99 \%$ occurrence in non-wetlands, NI $=$ not indicated.

Land use categories based upon subcatchment \% impervious surface. Urban $>30 \%(n=12)$, Rural $<10 \%(n=14)$, Suburban between 10 and $30 \%$ IS ( $n=15)$. $1 \& 2$ Basal area is the average within sites in each land use category including sites that did not contain the species (subscript 1) and including only sites containing the species (subscript 2). If the species was located in only one site, no standard error was given. 
Table 2-5: Sapling frequency and mean density (+ S.E.) in land use categories at the subcatchment scale.

\begin{tabular}{|c|c|c|c|c|c|c|c|}
\hline \multirow[b]{2}{*}{ Species } & \multirow[b]{2}{*}{$\begin{array}{l}\text { Wetland } \\
\text { Ind. } \\
\text { Status* }\end{array}$} & \multicolumn{2}{|c|}{ Urban } & \multicolumn{2}{|c|}{ Suburban } & \multicolumn{2}{|c|}{ Rural } \\
\hline & & Freq & $\begin{array}{l}\text { Mean } \\
\text { Density } \\
\text { (no./ha) }\end{array}$ & Freq & $\begin{array}{l}\text { Mean } \\
\text { Density } \\
\text { (no./ha) }\end{array}$ & Freq & $\begin{array}{l}\text { Mean } \\
\text { Density } \\
\text { (no./ha) }\end{array}$ \\
\hline Acer negundo & FAC & 0.25 & $97(71)$ & 0.13 & $167(155)$ & 0.21 & $45(27)$ \\
\hline Acer saccharum & FACU & 0.08 & $83(83)$ & 0.07 & $78(78)$ & 0 & 0 \\
\hline Carya cordiformis & FACU & 0.08 & $14(14)$ & 0.13 & $49(38)$ & 0 & 0 \\
\hline Celtis occidentalis & FACU & 0.33 & $519(342)$ & 0.20 & $45(26)$ & 0.14 & $61(46)$ \\
\hline Cornus sericea & FACW & 0.08 & $19(19)$ & 0 & 0 & 0 & 0 \\
\hline Fraxinus americana & FACU & 0.08 & $28(28)$ & 0.20 & $59(39)$ & 0.14 & $25(18)$ \\
\hline $\begin{array}{l}\text { Fraxinus } \\
\text { pennsylvanica }\end{array}$ & FACW & 0.08 & $23(23)$ & 0.07 & $139(139)$ & 0 & 0 \\
\hline $\begin{array}{l}\text { Fraxinus } \\
\text { quadrangulata }\end{array}$ & NI & 0.08 & $35(35)$ & 0.07 & $23(23)$ & 0 & 0 \\
\hline Maclura pomifera & UPL & 0.08 & $5(5)$ & 0 & 0 & 0 & 0 \\
\hline Morus rubra & FACU & 0.17 & $28(19)$ & 0 & 0 & 0 & 0 \\
\hline Thuja occidentalis & FACW & 0.08 & $5(5)$ & 0 & 0 & 0 & 0 \\
\hline Aesculus glabra & FACU & 0 & 0 & 0.07 & $11(11)$ & 0.14 & $42(36)$ \\
\hline Asimina triloba & FACU & 0 & 0 & 0.20 & $606(324)$ & 0.43 & $287(168)$ \\
\hline Cercis canadensis & FACU & 0 & 0 & 0.07 & $12(12)$ & 0 & 0 \\
\hline Cornus spp. & & 0 & 0 & 0.07 & $111(111)$ & 0 & 0 \\
\hline Prunus serotina & FACU & 0 & 0 & 0.07 & $11(11)$ & 0 & 0 \\
\hline Quercus rubra & FACU & 0 & 0 & 0.07 & $12(12)$ & 0 & 0 \\
\hline Staphylea trifolia & $\mathrm{FAC}$ & 0 & 0 & 0.07 & $667(667)$ & 0 & 0 \\
\hline Tilia americana & FACU & 0 & 0 & 0.07 & $12(12)$ & 0 & 0 \\
\hline Ulmus rubra & FAC & 0 & 0 & 0.13 & $92(72)$ & 0.21 & $30(17)$ \\
\hline $\begin{array}{l}\text { Juglans nigra } \\
\text { Robinia }\end{array}$ & FACU & 0 & 0 & 0 & 0 & 0.07 & $6(6)$ \\
\hline pseudoacacia & FAC & 0 & 0 & 0 & 0 & 0.07 & $60(60)$ \\
\hline \multirow{2}{*}{\multicolumn{8}{|c|}{$\begin{array}{l}\text { SUM } \\
* \text { Wetland indicator status - Frequency of occurrence in wetlands and non-wetlands: OBL }>99 \% \\
\text { in wetlands, FACW } 67-99 \% \text { in wetlands, FAC equally likely to occur in wetlands and non- }\end{array}$}} \\
\hline & & & & & & & \\
\hline $\begin{array}{l}\text { Land use categories } \\
(\mathrm{n}=12) \text {, Rural }<10\end{array}$ & $\begin{array}{l}d \text { upon } \\
=14) \text {, }\end{array}$ & np & $\begin{array}{l}\text { is surfa } \\
\text { tween }\end{array}$ & the & $\begin{array}{l}\text { atchment } \\
(\mathrm{n}=15)\end{array}$ & le. $L$ & $n>30 \%$ \\
\hline
\end{tabular}


Table 2-6: Tree seedling frequency and mean density (+ S.E.) in land use categories at the subcatchment scale.

\begin{tabular}{|c|c|c|c|c|c|c|c|}
\hline \multirow{2}{*}{ Species } & \multirow[b]{2}{*}{$\begin{array}{c}\text { Wetland } \\
\text { Ind. } \\
\text { Status* }\end{array}$} & \multicolumn{2}{|r|}{ Urban } & \multicolumn{2}{|c|}{ Suburban } & \multicolumn{2}{|r|}{ Rural } \\
\hline & & Freq & $\begin{array}{c}\text { Mean } \\
\text { Density } \\
\text { (stems/ha) }\end{array}$ & Freq & $\begin{array}{c}\begin{array}{c}\text { Mean } \\
\text { Density } \\
\text { (stems/ha) }\end{array} \\
\end{array}$ & Freq & $\begin{array}{c}\text { Mean } \\
\text { Density } \\
\text { (stems/ha) }\end{array}$ \\
\hline Acer negundo & FAC & 0.42 & $3935(3060)$ & 0.60 & $1167(362)$ & 0.43 & $1210(573)$ \\
\hline Aesculus glabra & FACU & 0.08 & $139(139)$ & 0.20 & $222(128)$ & 0.14 & $60(40)$ \\
\hline Betula alleghaniensis & $\mathrm{FACU}$ & 0.08 & $23(23)$ & 0 & 0 & 0 & 0 \\
\hline Celtis occidentalis & FACU & 0.33 & $1389(651)$ & 0.60 & $2556(1520)$ & 0.36 & $843(486)$ \\
\hline Fraxinus americana & FACU & 0.25 & $255(151)$ & 0.20 & $283(157)$ & 0.21 & $1647(1098)$ \\
\hline Fraxinus spp. & & 0.08 & $69(69)$ & 0 & 0 & 0 & 0 \\
\hline Gleditsia tricanthos & FAC & 0.08 & $139(139)$ & 0 & 0 & 0 & 0 \\
\hline Morus rubra & FACU & 0.17 & $93(71)$ & 0 & 0 & 0 & 0 \\
\hline Platanus occidentalis & FACW & 0.08 & $69(69)$ & 0.07 & $111(111)$ & 0.07 & $79(79)$ \\
\hline Acer saccharum & FACU & 0 & 0 & 0.27 & $833(467)$ & 0.21 & $992(654)$ \\
\hline Asimina triloba & FACU & 0 & 0 & 0.20 & $1444(977)$ & 0.29 & $327(152)$ \\
\hline Carya cordiformis & FACU & 0 & 0 & 0.07 & $222(222)$ & 0.07 & $60(60)$ \\
\hline Cercis canadensis & FACU & 0 & 0 & 0.07 & $111(111)$ & 0 & 0 \\
\hline $\begin{array}{l}\text { Cornus drummondii } \\
\text { Fraxinus }\end{array}$ & FAC & 0 & 0 & 0.07 & $222(222)$ & 0 & 0 \\
\hline $\begin{array}{l}\text { pennsylvanica } \\
\text { Fraxinus }\end{array}$ & FACW & 0 & 0 & 0.07 & $1222(1222)$ & 0 & 0 \\
\hline $\begin{array}{l}\text { quadrangulata } \\
\text { Liriodendron }\end{array}$ & NI & 0 & 0 & 0.07 & $242(242)$ & 0.07 & $159(159)$ \\
\hline tulipifera & $\mathrm{FACU}$ & 0 & 0 & 0.13 & $167(121)$ & 0 & 0 \\
\hline Prunus serotina & FACU & 0 & 0 & 0.07 & $424(424)$ & 0 & 0 \\
\hline Quercus shumardii & $\mathrm{FAC}$ & 0 & 0 & 0.07 & $61(61)$ & 0 & 0 \\
\hline Tilia americana & FACU & 0 & 0 & 0.07 & $56(56)$ & 0 & 0 \\
\hline Ulmus rubra & $\mathrm{FAC}$ & 0 & 0 & 0.07 & $424(424)$ & 0.14 & $149(120)$ \\
\hline Acer spp. & & 0 & 0 & 0 & 0 & 0.14 & $89(64)$ \\
\hline Juglans cinerea & FACU & 0 & 0 & 0 & 0 & 0.07 & $89(89)$ \\
\hline $\begin{array}{l}\text { Quercus rubra } \\
\text { Robinia }\end{array}$ & FACU & 0 & 0 & 0 & 0 & 0.07 & $159(159)$ \\
\hline pseudoacacia & FAC & 0 & 0 & 0 & 0 & 0.07 & $30(30)$ \\
\hline SUM & & & $6111(1114)$ & & $9768(880)$ & & $5893(668)$ \\
\hline
\end{tabular}

*Wetland indicator status - Frequency of occurrence in wetlands and non-wetlands: OBL $>99 \%$ in wetlands, FACW $67-99 \%$ in wetlands, FAC equally likely to occur in wetlands and non-wetlands, FACU $67-99 \%$ occurrence in non-wetlands, UPL $>99 \%$ occurrence in non-wetlands, NI $=$ not indicated

Land use categories based upon \% impervious surface at the subcatchment scale. Urban $>30 \%(n=12)$, Rural $<10 \%(n=14)$, Suburban between 10 and $30 \%$ IS $(n=15)$. 
Table 2-7: Shrub frequency and mean density (+ S.E.) in land-use categories at the subcatchment scale.

\begin{tabular}{|c|c|c|c|c|c|c|c|}
\hline \multirow[b]{2}{*}{ Species } & \multirow[b]{2}{*}{$\begin{array}{l}\text { Wetland } \\
\text { Ind. } \\
\text { Status* }\end{array}$} & \multicolumn{2}{|r|}{ Urban } & \multicolumn{2}{|c|}{ Suburban } & \multicolumn{2}{|r|}{ Rural } \\
\hline & & Freq & $\begin{array}{c}\text { Mean } \\
\text { Density } \\
\text { (stems/ha) }\end{array}$ & Freq & $\begin{array}{c}\text { Mean } \\
\text { Density } \\
\text { (stems/ha) }\end{array}$ & Freq & $\begin{array}{c}\text { Mean Density } \\
\text { (stems/ha) }\end{array}$ \\
\hline Cornus spp. & & 0.08 & $799(799)$ & 0 & 0 & 0 & 0 \\
\hline Euonymus alatus & NI & 0.17 & $69(48)$ & 0.33 & $389(226)$ & 0.07 & $32(32)$ \\
\hline $\begin{array}{l}\text { Hibiscus syriacus } \\
\text { Hypericum }\end{array}$ & $\mathrm{NI}$ & 0.08 & $660(660)$ & 0 & 0 & 0 & 0 \\
\hline $\begin{array}{l}\text { prolificum } \\
\text { Lagerstroemia }\end{array}$ & FACU & 0.08 & $14(14)$ & 0 & 0 & 0 & 0 \\
\hline indica & NI & 0.08 & $417(417)$ & 0 & 0 & 0 & 0 \\
\hline Ligustrum sinense & $\mathrm{FACU}$ & 0.08 & $389(389)$ & 0.40 & $2456(1484)$ & 0.29 & $1345(1160)$ \\
\hline Lindera benzoin & FACW & 0.25 & $458(309)$ & 0.27 & $728(418)$ & 0.57 & $1712(665)$ \\
\hline Lonicera maackii & NI & 1.00 & 8005 (2023) & 0.60 & $2767(1401)$ & 0.86 & $2618(783)$ \\
\hline $\begin{array}{l}\text { Rosa palustris } \\
\text { Amelanchier }\end{array}$ & $\mathrm{OBL}$ & 0.08 & $97(97)$ & 0 & 0 & 0 & 0 \\
\hline arborea & FAC & 0 & 0 & 0.07 & $56(56)$ & 0 & 0 \\
\hline Cornus racemosa & NI & 0 & 0 & 0.07 & $89(89)$ & 0.07 & $42(42)$ \\
\hline Hibiscus syriacus & NI & 0 & 0 & 0.07 & $111(111)$ & 0 & 0 \\
\hline $\begin{array}{l}\text { Rubus spp. } \\
\text { Hydrangea }\end{array}$ & & 0 & 0 & 0.07 & $222(222)$ & 0.07 & $595(595)$ \\
\hline $\begin{array}{l}\text { arborescens } \\
\text { Symphoricarpos }\end{array}$ & FACU & 0 & 0 & 0 & 0 & 0.14 & $179(129)$ \\
\hline $\begin{array}{l}\text { orbiculatus } \\
\text { Viburnum }\end{array}$ & UPL & 0 & 0 & 0 & 0 & 0.21 & $4536(4273)$ \\
\hline acerifolium & UPL & 0 & 0 & 0 & 0 & 0.07 & $60(60)$ \\
\hline SUM & & & $10907(1643)$ & & $6817(1015)$ & & $11118(1322)$ \\
\hline
\end{tabular}

*Wetland indicator status - Frequency of occurrence in wetlands and non-wetlands: OBL $>99 \%$ in wetlands, FACW $67-99 \%$ in wetlands, FAC equally likely to occur in wetlands and non-wetlands, FACU $67-99 \%$ occurrence in non-wetlands, UPL $>99 \%$ occurrence in non-wetlands, $\mathrm{NI}=$ not indicated.

Land use categories based upon \% impervious surface at the subcatchment scale. Urban $>30 \%(n=12)$, Rural $<10 \%(n=14)$, Suburban between 10 and $30 \%$ IS $(n=15)$. 
Table 2-8: Tree diameter distribution in riparian zones by land-use category at the subcatchment scale.

\begin{tabular}{|c|c|c|c|c|c|c|c|c|}
\hline $\begin{array}{l}\text { Land } \\
\text { Use1 }\end{array}$ & Species & & & Diam & r Cla & $(\mathrm{cm}$ & & \\
\hline & & 2.5 & $5-$ & $10-$ & $25-$ & $30-$ & $35-$ & \\
\hline & & 5 & 10 & 25 & 30 & 35 & 40 & $>40 \#$ \\
\hline & Acer negundo & 12 & 25 & 37 & & 6 & & \\
\hline & Acer saccharum & 6 & & 19 & & 12 & 6 & \\
\hline & Aesculus glabra & 25 & 19 & 6 & & & & \\
\hline & Asimina triloba & 56 & 6 & & & & & \\
\hline & Carpinus caroliniana & & 6 & & & & & \\
\hline & Celtis occidentalis & 12 & 6 & 6 & & & & \\
\hline & Cercis canadensis & & 6 & 6 & & & & \\
\hline $\bar{\pi}$ & Cornus florida & 12 & & & & & & \\
\hline$\underline{\tilde{z}}$ & Fraxinus americana & 12 & 19 & 12 & 6 & & & \\
\hline & Juglans nigra & & & & & & 12 & $19(53)$ \\
\hline & Juniperus virginiana & & 6 & 12 & 6 & 6 & & \\
\hline & Maclura pomifera & 12 & 31 & 6 & & 6 & & $6(47)$ \\
\hline & Platanus occidentalis & & & & & 6 & & $12(68)$ \\
\hline & Prunus serotina & & 6 & & & & & \\
\hline & Quercus alba & & & & 6 & & & \\
\hline & Ulmus rubra & 12 & 25 & & 6 & & & \\
\hline Total & & 161 & 155 & 106 & 25 & 37 & 19 & 37 \\
\hline & Acer negundo & 49 & 49 & 81 & 24 & 8 & & \\
\hline & Acer saccharinum & & 8 & 16 & 8 & & & \\
\hline & Acer saccharum & 16 & 16 & 49 & . & & & \\
\hline & Aesculus glabra & & 8 & & & & & \\
\hline & Asimina triloba & 16 & 8 & & & & & \\
\hline & Celtis occidentalis & 33 & & 8 & & & & \\
\hline$\stackrel{\overline{0}}{0}$ & Cornus alternifolia & 8 & & 8 & & & & \\
\hline$\Xi$ & Fraxinus americana & 8 & & 24 & 8 & & & $8(45.5)$ \\
\hline$\vec{n}$ & Fraxinus pennsylvanica & 8 & & & & & & \\
\hline & Juglans nigra & 8 & & 8 & & & & \\
\hline & Morus alba* & & 8 & 8 & 8 & & & \\
\hline & Platanus occidentalis & & 8 & & & & & \\
\hline & Staphylea trifolia & 16 & & & & & & \\
\hline & Tilia americana & 8 & & 41 & & & & \\
\hline & Ulmus rubra & 16 & 49 & 57 & & 8 & & \\
\hline Total & & 187 & 154 & 301 & 49 & 16 & 0 & 8 \\
\hline
\end{tabular}


Table 2-8 (cont.)

\begin{tabular}{|c|c|c|c|c|c|c|c|c|}
\hline & Acer negundo & 15 & 146 & 66 & 7 & & & $7(93)$ \\
\hline & Acer saccharinum & & & & & 7 & & \\
\hline & Acer saccharum & 22 & 7 & 15 & & & & \\
\hline & Aesculus glabra & & & & 7 & & & \\
\hline & Betula alleghaniensis & 7 & & & & & & \\
\hline & Carya cordiformis & & 7 & & 7 & & 7 & \\
\hline & Celtis occidentalis & 7 & 22 & 29 & 7 & 7 & & $14(53)$ \\
\hline & Fraxinus americana & & 22 & 22 & & & & \\
\hline $\bar{\xi}$ & Fraxinus pennsylvanica & 7 & 7 & & & & & \\
\hline ำ & Juglans nigra & & & 29 & 15 & & & \\
\hline & Maclura pomifera & & 7 & 7 & & & & \\
\hline & Morus alba* & & 22 & 58 & & & & \\
\hline & Morus rubra & & 7 & 7 & & & & \\
\hline & Picea pungens & & 7 & & & & & \\
\hline & Platanus occidentalis & & & & & 7 & & $14(93)$ \\
\hline & Prunus cerasifera* & & & 7 & & & & \\
\hline & Quercus rubra & 7 & & & & & & \\
\hline & Thuja occidentalis & 7 & & & & & & \\
\hline & Ulmus rubra & 22 & 15 & & 7 & & & \\
\hline Total & & 95 & 270 & 241 & 51 & 22 & 7 & 36 \\
\hline
\end{tabular}

ILand use categories based upon \% impervious surface at the subcatchment scale. Urban $>30 \%(n=12)$, Rural $<10 \%(n=$ $14)$, Suburban between 10 and $30 \%$ IS $(n=15)$.

*Non-native species

\# Number in parentheses is the largest diameter tree within the size class. 
Table 2-9: ANOVA of woody plant richness by plant type among land-use categories at the subcatchment scale. The seedling category includes tree seedlings only.

\begin{tabular}{lcc} 
Scale & $\begin{array}{c}\text { Plant } \\
\text { type }\end{array}$ & p-value \\
\hline Near-stream & & \\
plots & Tree & 0.46 \\
& Saplings & 0.32 \\
& Seedlings & $\mathbf{0 . 0 2 6}$ \\
& Shrubs & 0.87 \\
\hline Sites & Trees & 0.82 \\
& Saplings & 0.8 \\
& Seedlings & $\mathbf{0 . 0 3 4}$ \\
& Shrubs & 0.89 \\
$*_{p}=0.05$ & &
\end{tabular}

Richness = \# spp./ log(sampled area $)$

Site richness determined by treating all plots at multi-plot sites as one sampling unit. 
Table 2-10: Tukeys HSD pairwise comparisons of tree seedling richness by land-use category at the subcatchment scale.

\begin{tabular}{lccc} 
Scale & Pairing & Difference & Adj. p-value \\
\hline Near-stream plots & Suburban-Rural & 1.21 & 0.17 \\
& Urban-Rural & -0.66 & 0.61 \\
& Urban-Suburban & -1.87 & $\mathbf{0 . 0 2 4}$ \\
\hline \multirow{2}{*}{ Sites } & Suburban-Rural & 1.05 & 0.26 \\
& Urban-Rural & -0.80 & 0.49 \\
& Urban-Suburban & -1.85 & $\mathbf{0 . 0 2 8}$
\end{tabular}


Table 2-11: Woody plant species composition for the most common species and significant indicator values for a 3-cluster configuration using optimal partitioning. Frequency values were calculated based on the proportion of sites within the cluster in which the species occurred. Indicator values were calculated using indicator species analysis, where a value of 0 means the species is never present in the cluster and a value of 1 means the species is always present and exclusive to the cluster. Probabilities for the indicator values were determined using a Monte Carlo randomization technique.

Compare with the 5-cluster configuration in Table 2-12. Celtis occidentalis was included despite having no significant indicator value since it occurred most frequently (although not exclusively) in that cluster among the three growth stages.

\begin{tabular}{|c|c|c|c|c|c|}
\hline Cluster & Species & $\begin{array}{c}\text { Common } \\
\text { name }\end{array}$ & Frequency & $\begin{array}{c}\text { Indicator } \\
\text { value }\end{array}$ & probability \\
\hline 1 & $\begin{array}{l}\text { Symphoricarpos } \\
\text { orbiculata }\end{array}$ & Coralberry & 0.66 & 0.66 & 0.001 \\
\hline \multirow[t]{5}{*}{2} & Lindera benzoin & Spicebush & 0.8 & 0.76 & 0.004 \\
\hline & Asimina triloba & Pawpaw & & & \\
\hline & & -Trees & 0.41 & 0.42 & 0.023 \\
\hline & & -Saplings & 0.66 & 0.67 & 0.01 \\
\hline & & -Seedlings & 0.5 & 0.48 & 0.032 \\
\hline \multirow[t]{5}{*}{3} & Lonicera maackii & $\begin{array}{l}\text { Amur } \\
\text { honeysuckle }\end{array}$ & 0.88 & 0.84 & 0.001 \\
\hline & Celtis occidentalis & Hackberry & & & \\
\hline & & -Trees & 0.26 & - & - \\
\hline & & -Saplings & 0.34 & - & - \\
\hline & & -Seedlings & 0.57 & - & - \\
\hline
\end{tabular}


Table 2-12: Woody plant species composition and significant indicator values (IV) for 5 cluster configuration using optimal partitioning. Frequency values were calculated based on the proportion of sites within the cluster in which the species occurred. Indicator values were calculated using indicator species analysis, where a value of 0 means the species is never present in the cluster and a value of 1 means the species is always present and exclusive to the cluster. Probabilities for the indicator values were determined using a Monte Carlo randomization technique. Compare with the 3-cluster configuration in Table 2-11. Other species with frequencies $\geq 0.4$ were included to illustrate species differences and similarities among groups.

\begin{tabular}{|c|c|c|c|c|c|c|}
\hline Cluster & Species & $\begin{array}{l}\text { Common } \\
\text { name }\end{array}$ & Freq. & IV & Prob. & $\begin{array}{l}\text { Other spp. (freq. } \geq \\
0.4 \text { ) }\end{array}$ \\
\hline \multirow[t]{5}{*}{1} & $\begin{array}{l}\text { Lindera } \\
\text { benzoin }\end{array}$ & Spicebush & 1 & 0.7 & 0.001 & $\begin{array}{l}\text { Celtis occidentalis, } \\
\text { Ulmus rubra }\end{array}$ \\
\hline & $\begin{array}{c}\text { Asimina } \\
\text { triloba }\end{array}$ & Pawpaw & & & & $\begin{array}{l}\text { Aesculus glabra } \\
\text { seedlings, } \\
\text { Ligustrum sinense }\end{array}$ \\
\hline & & Trees & 0.55 & 0.56 & 0.01 & \\
\hline & & Saplings & 1 & 1 & 0.001 & \\
\hline & & Seedlings & 0.66 & 0.59 & 0.02 & \\
\hline 2 & $\begin{array}{c}\text { Acer } \\
\text { negundo }\end{array}$ & Boxelder & 1 & 0.68 & 0.001 & $\begin{array}{l}\text { Celtis occidentalis } \\
\text { seedlings, Lindera } \\
\text { benzoin }\end{array}$ \\
\hline 3 & $\begin{array}{l}\text { Lonicera } \\
\text { maackii }\end{array}$ & $\begin{array}{l}\text { Amur } \\
\text { honeysuckle }\end{array}$ & 1 & 0.57 & 0.001 & $\begin{array}{l}\text { Acer negundo } \\
\text { seedlings, Celtis } \\
\text { occidentalis } \\
\text { saplings/seedlings }\end{array}$ \\
\hline 4 & & $\begin{array}{l}\text { Outlier } \\
\text { cluster }\end{array}$ & & & & \\
\hline 5 & $\begin{array}{c}\text { Acer } \\
\text { negundo }\end{array}$ & $\begin{array}{l}\text { Boxelder } \\
\text { (seedlings) }\end{array}$ & 0.8 & 0.5 & 0.03 & $\begin{array}{l}\text { Symphoricarpos } \\
\text { orbiculata, } \\
\text { Ligustrum sinense, } \\
\text { Ulmus rubra } \\
\text { saplings }\end{array}$ \\
\hline
\end{tabular}


Table 2-13: Environmental and National Land Cover Database (NLCD) metrics used for non-metric multidimensional scaling.

Environmental variables

-Bank height adjacent to the site

$-\%$ impervious surface: subcatchment, 1-km buffer, 500-m buffer

-Area sampled

-Stream order

-Catchment Area

-Property value

\section{NLCD values $(500-m)$}

-Open-space developed

-High-, medium, low-intensity developed

-Deciduous forest

-Mixed forest

-Herbaceous forest

-Crops

-Pasture

-Wetland 


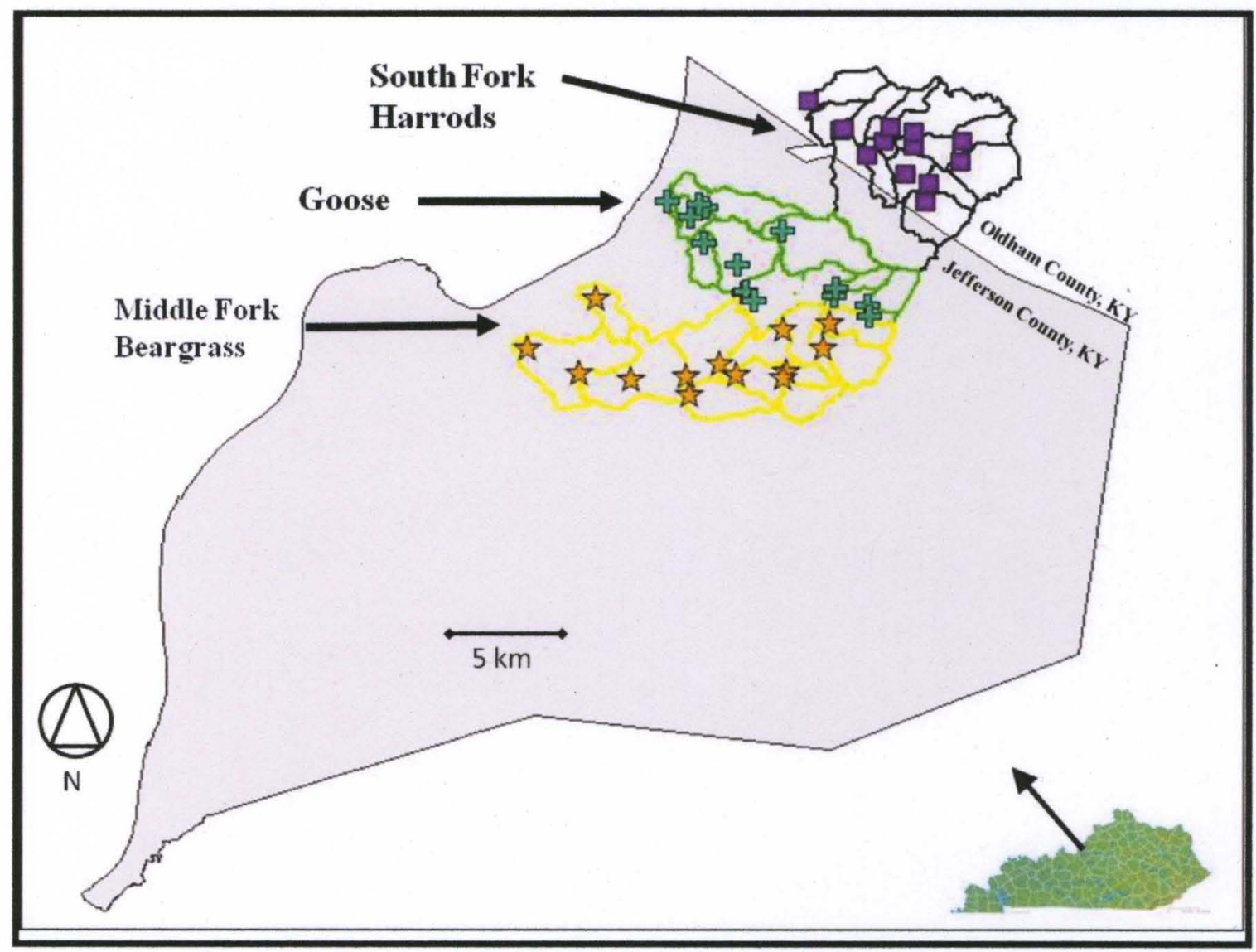

Figure 2-1: Schematic map of the three watersheds in Jefferson and Oldham counties in Kentucky. Research sites are indicated with markers. 


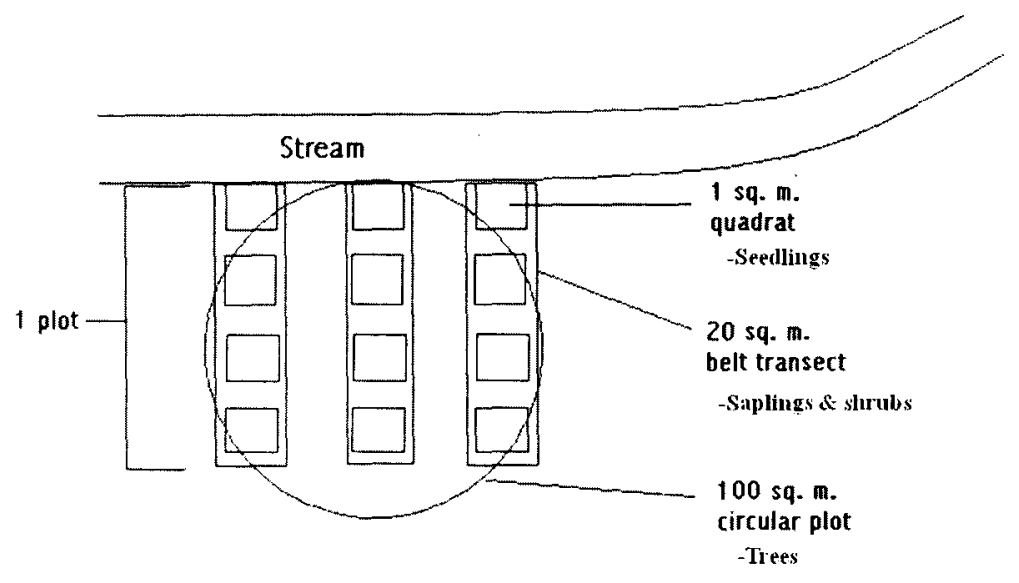

(a)

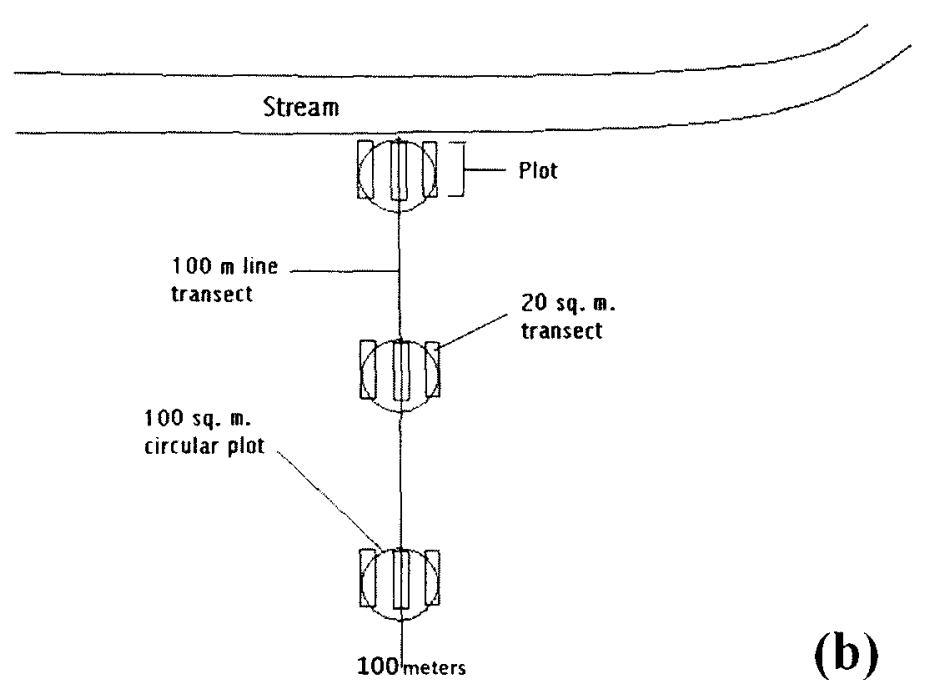

Figure 2-2: Plot and site design for sampling adult trees $(\geq 2.54-\mathrm{cm} \mathrm{DBH})$, saplings, tree seedlings, and shrubs. (a) Each site consisted of at least one $100-\mathrm{m}^{2}$ circular plot for sampling tree counts and $\mathrm{DBH}$, except in the cases when the riparian width could not accommodate a complete circular plot. Nested within the circular plot were three belt transects measuring $10 \times 2 \mathrm{~m}$ with midlines spaced four meters apart for counting saplings and shrub stems. Quadrats measuring $1 \times 1 \mathrm{~m}$ were nested within the belt transects for determining percent cover and counts of tree seedlings. Adjacent quadrat spacing within belt transects was three meters at the midpoint. (b) Where riparian width was sufficient, sites consisted of a midline transect stretched the length of the riparian zone up to 100 meters with up to 3 plots per transect with midpoints spaced $40-\mathrm{m}$ apart. 


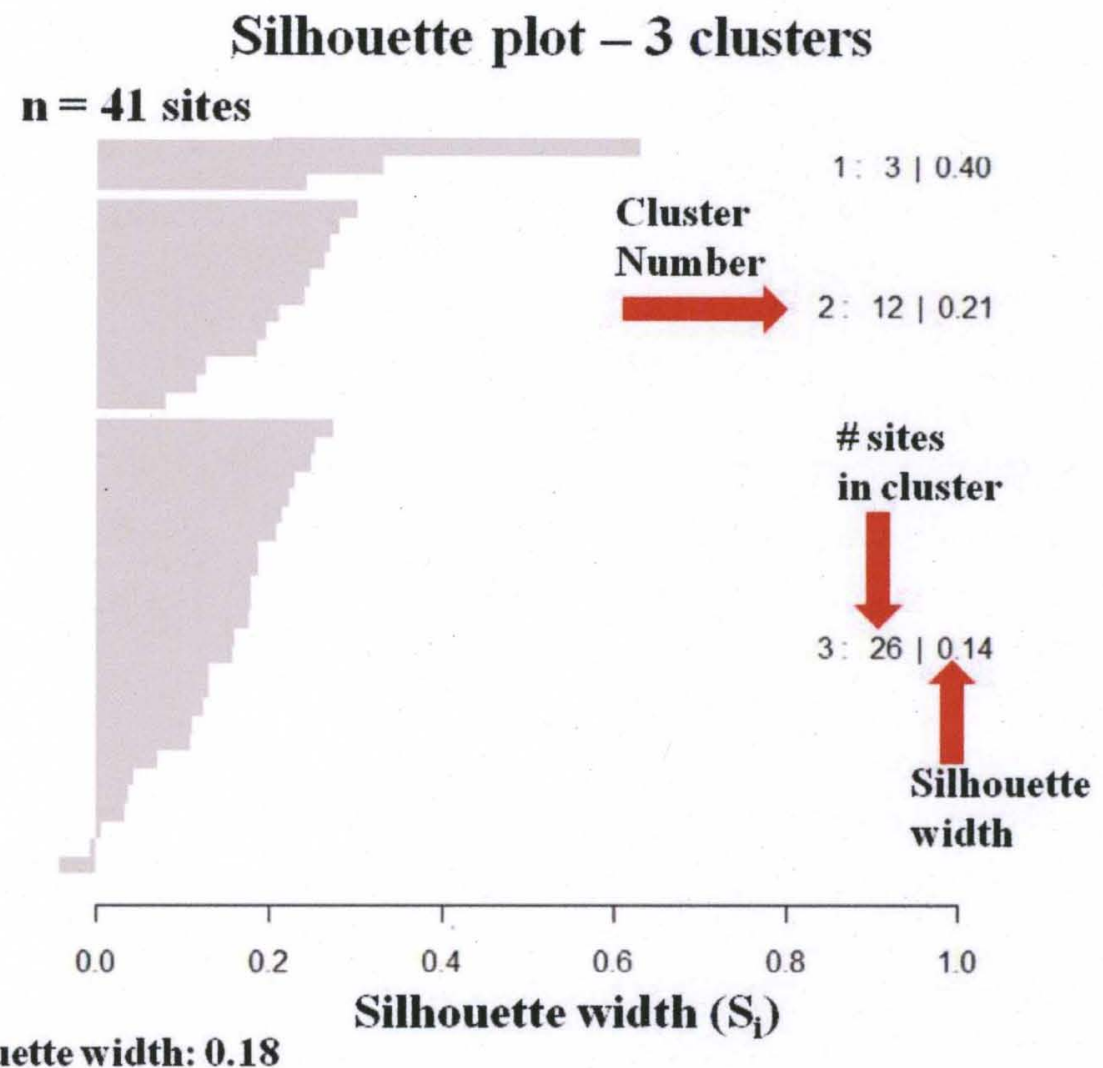

Average silhouette width: 0.18

Figure 2-3: Silhouette plot of a cluster analysis (optimal partitioning using Bray-Curtis distance) resulting in three groupings of research sites. Sites within clusters are represented by gray bars, the length of each demonstrating the silhouette width (value on the X-axis) of that particular site within the cluster. Cluster numbers, number of sites within the cluster, and cluster widths are to the right of each cluster. Overall silhouette widths are located below the numerical axis. Explanation of values given in Methods. 


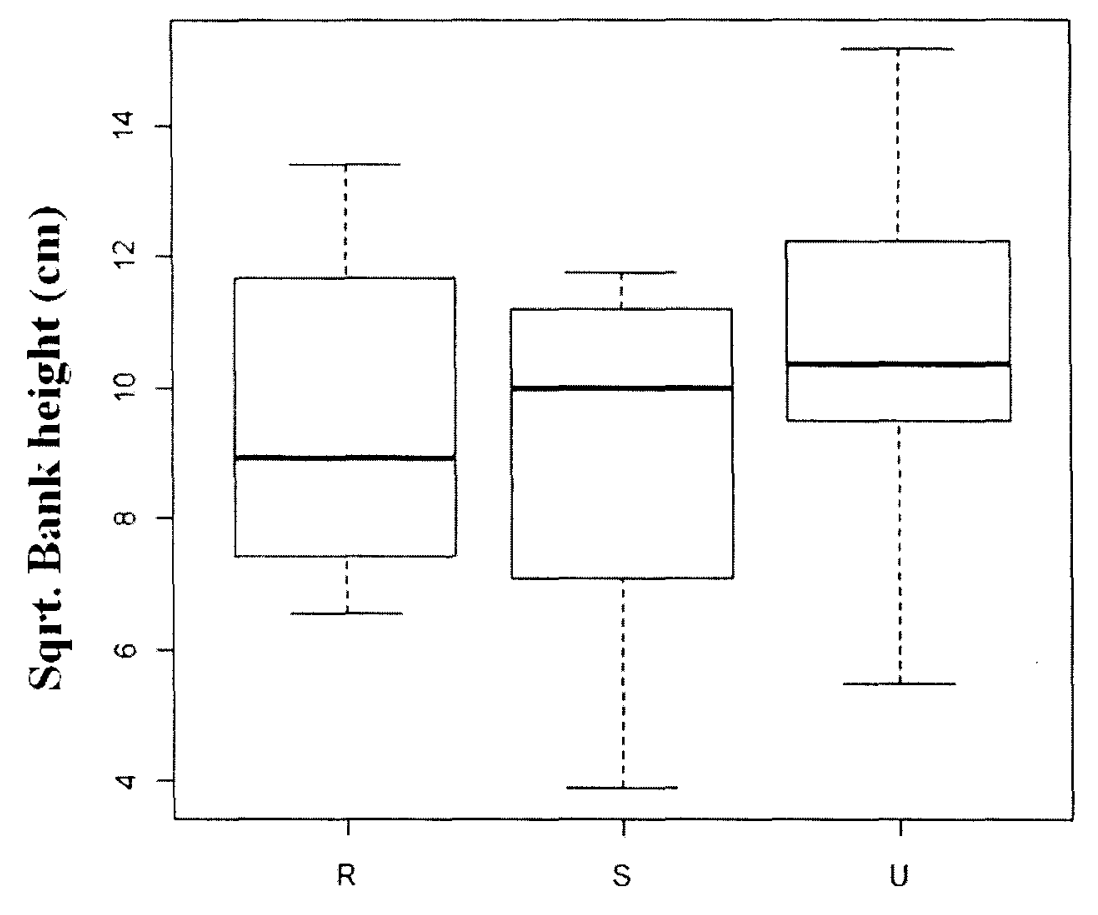

Land cover

Figure 2-4: Bank height measurements for land-use categories determined at the subcatchment scale $(\mathrm{R}=$ Rural, $\mathrm{n}=22 ; \mathrm{S}=$ Suburban, $\mathrm{n}=23 ; \mathrm{U}=$ Urban, $\mathrm{n}=22)$. The bold line indicates the median, boxes delineate $25^{\text {th }}$ and $75^{\text {th }}$ percentiles, and whiskers indicate minimum and maximum values. Mean urban bank height $(122-\mathrm{cm})$ was higher than suburban bank height $(86-\mathrm{cm} ; \mathrm{p}=0.068)$ and rural bank height $(96-\mathrm{cm})$. 


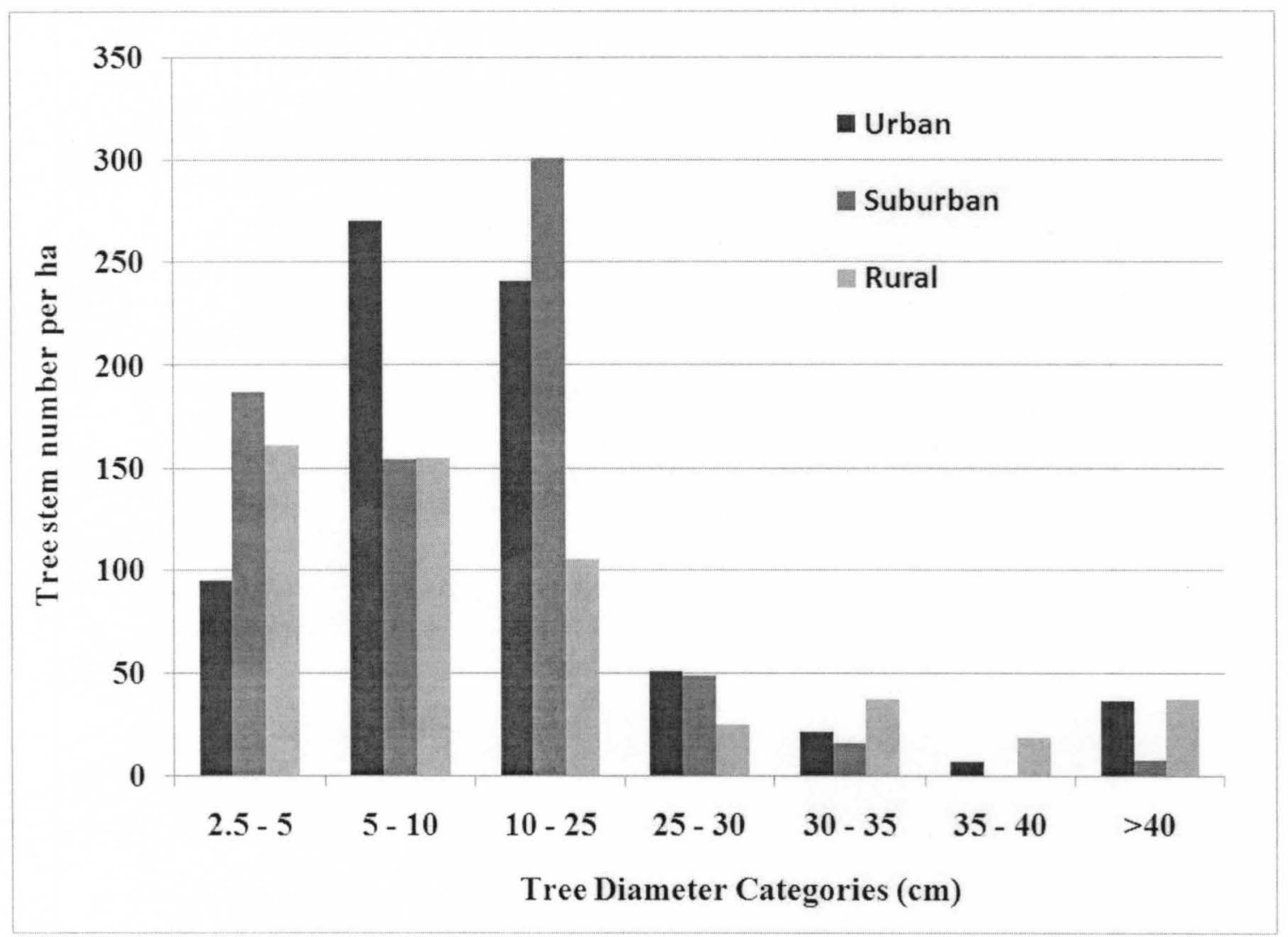

Figure 2-5: Tree diameter distributions by land-use category in riparian zones. Land-use categories were determined at the subcatchment scale. 


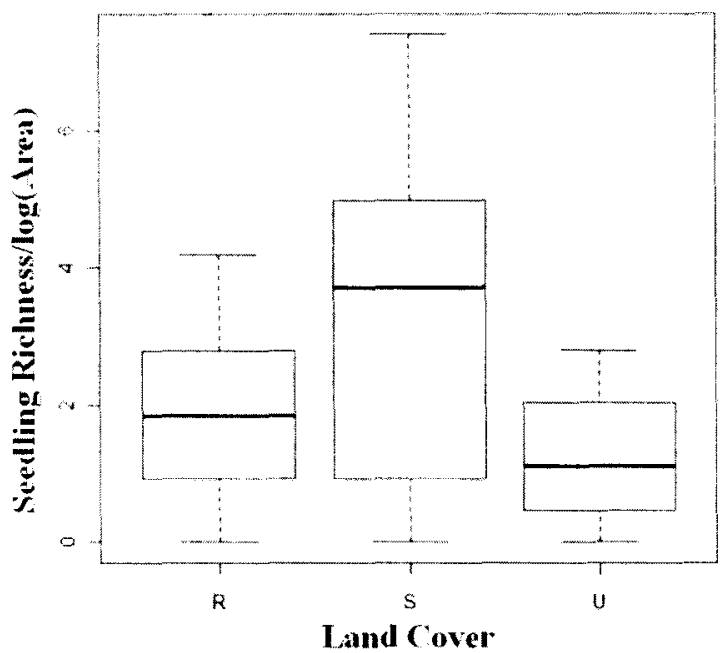

(a)

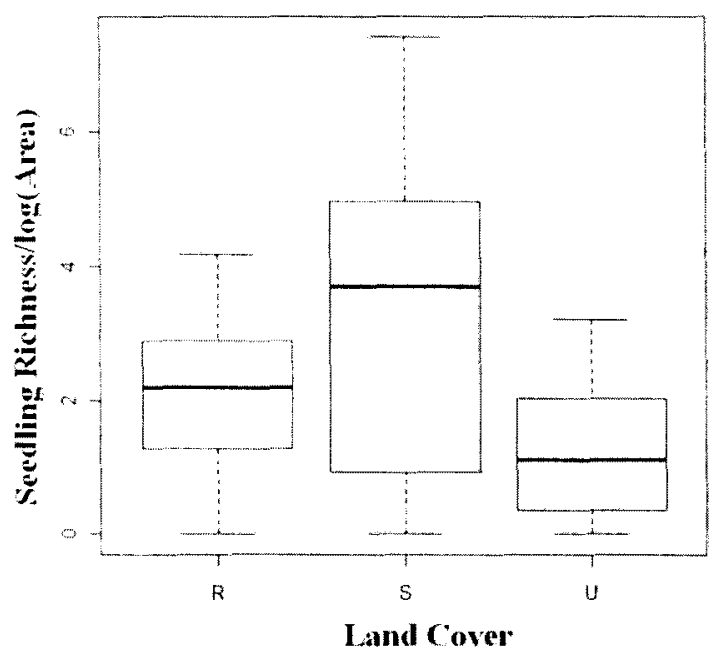

(b)

Figure 2-6: Tree seedling richness/ $\log$ (Area sampled) for (a) near-stream plots only ( $\mathrm{n}=$ 41 nearstream plots), and (b) sites ( $\mathrm{n}=41$ sites). In (b), plots at the multi-plot sites were combined and treated as one sampling unit. Land use assignment for both plot and multiplot sites was determined at the subcatchment scale $(\mathrm{R}=$ Rural, $\mathrm{S}=$ Suburban, $\mathrm{U}=$ Urban). The bold line indicates the median value, boxes delineate $25^{\text {th }}$ and $75^{\text {th }}$ percentiles, and whiskers indicate minimum and maximum values. Mean suburban tree seedling richness (Site and nearstream means $=2.8$ species per site and nearstream plot) was significantly higher than mean urban tree seedling richness ( nearstream mean $=1.3$ species) at $\mathrm{p}=0.05$ (nearstream plots only $\mathrm{p}=0.024$; all plots, $\mathrm{p}=$ $0.028)$. 


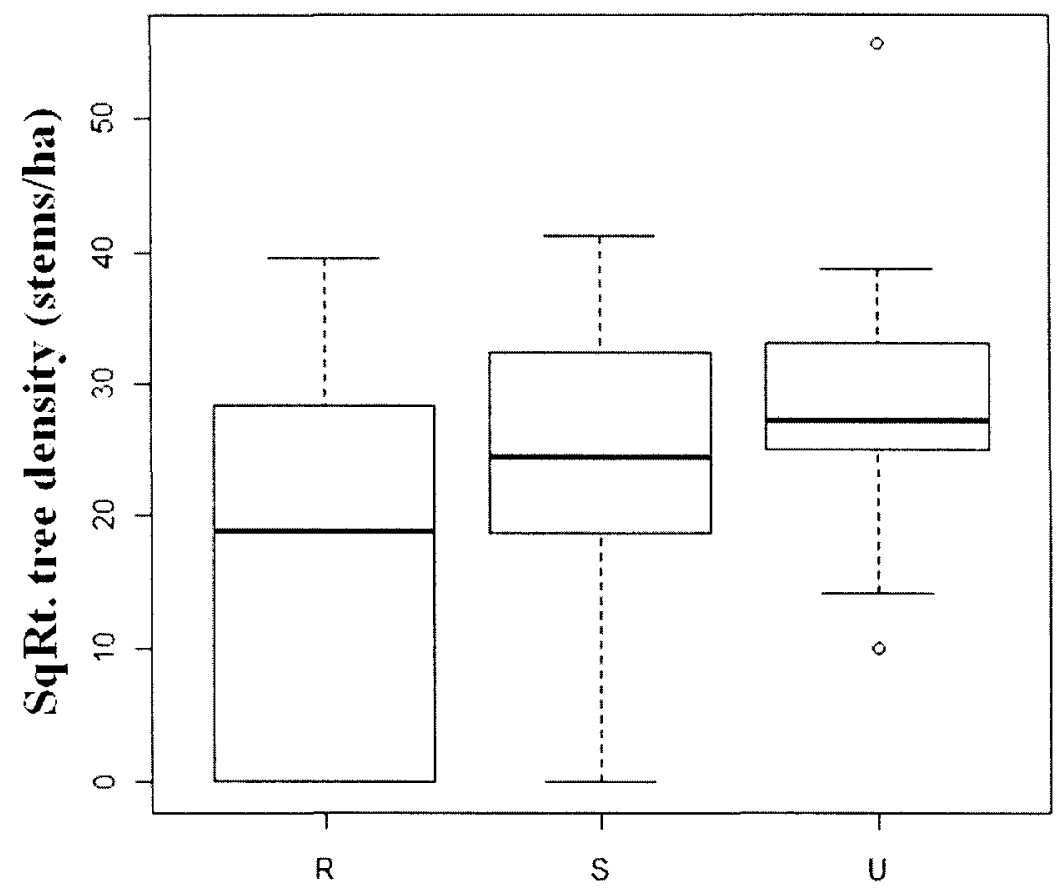

\section{Land cover}

Figure 2-7: Square root of the tree density averaged across all nearstream plots in each land-use category. Land use categories were determined at the subcatchment scale $(\mathrm{R}=$ Rural, $\mathrm{S}=$ Suburban, $\mathrm{U}=\mathrm{Urban}$ ). The bold line indicates the median, boxes delineate $25^{\text {th }}$ and $75^{\text {th }}$ percentiles, and whiskers indicate minimum and maximum values. Circular markers represent potential outliers, but were not excluded from analysis. Mean rural tree density (477 stems/ha) was nearly half that of urban tree density ( 954 stems/ha; $\mathrm{p}=$ $0.073)$. Mean suburban tree density was intermediate $(705 \mathrm{stems} / \mathrm{ha})$. 


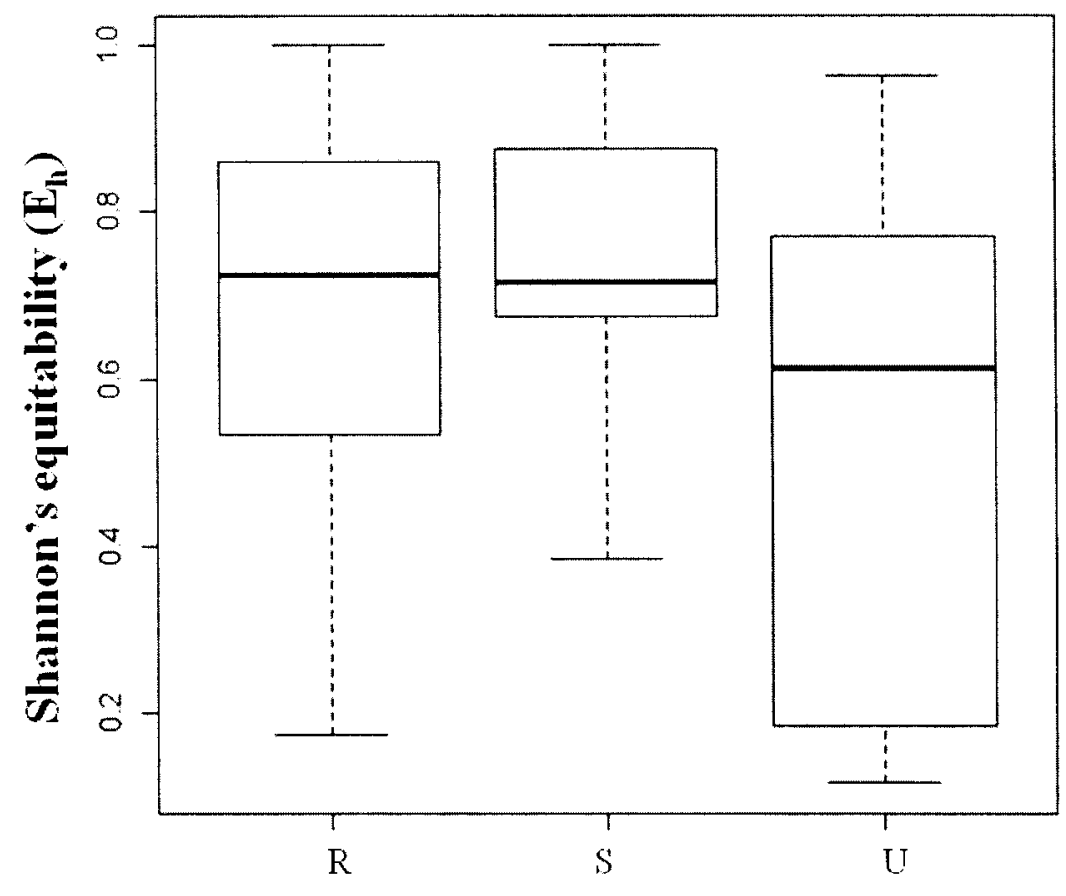

\section{Land cover}

Figure 2-8: Shannon's equitability index $\left(E_{h}\right)$ for all woody species using nearstream plots only for comparisons. Land-use categories were determined at the subcatchment scale $(\mathrm{R}=$ Rural, $\mathrm{S}=$ Suburban, $\mathrm{U}=\mathrm{Urban})$. The bold line indicates the median, boxes delineate $25^{\text {th }}$ and $75^{\text {th }}$ percentiles, and whiskers indicate minimum and maximum values. Mean urban woody plant evenness $(0.54)$ was significantly lower $(p=0.037)$ than suburban community evenness $(0.77)$, while mean rural woody plant evenness $(0.68)$ was similar to that of suburban, but more variable. 


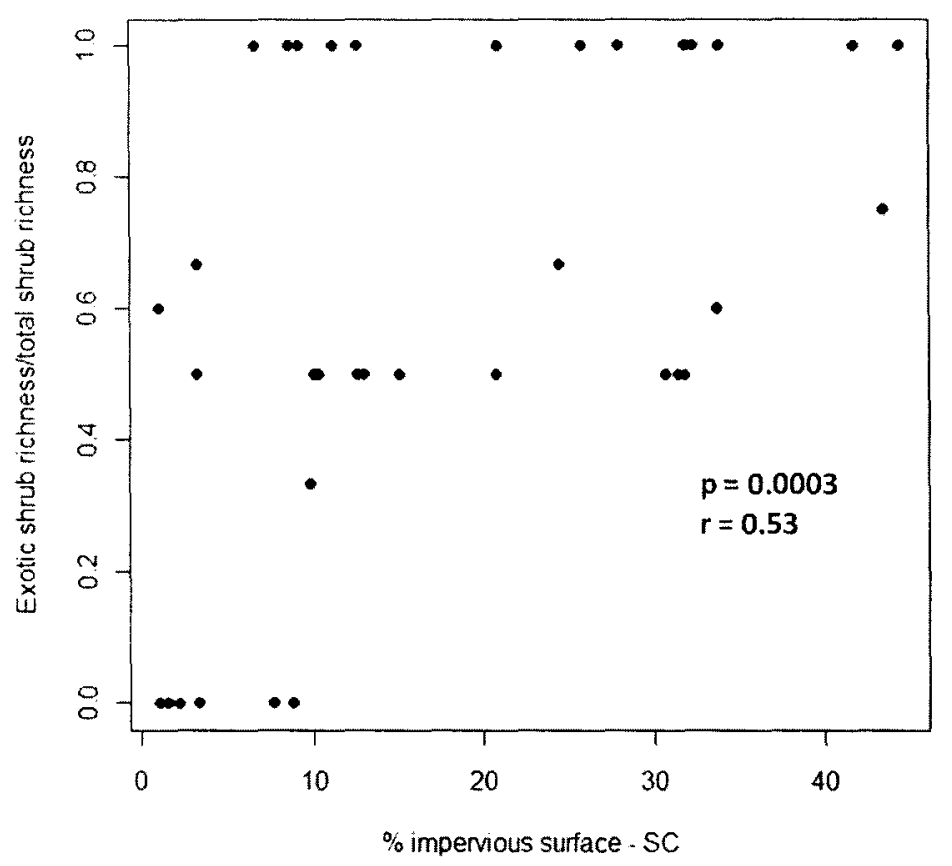

(a)

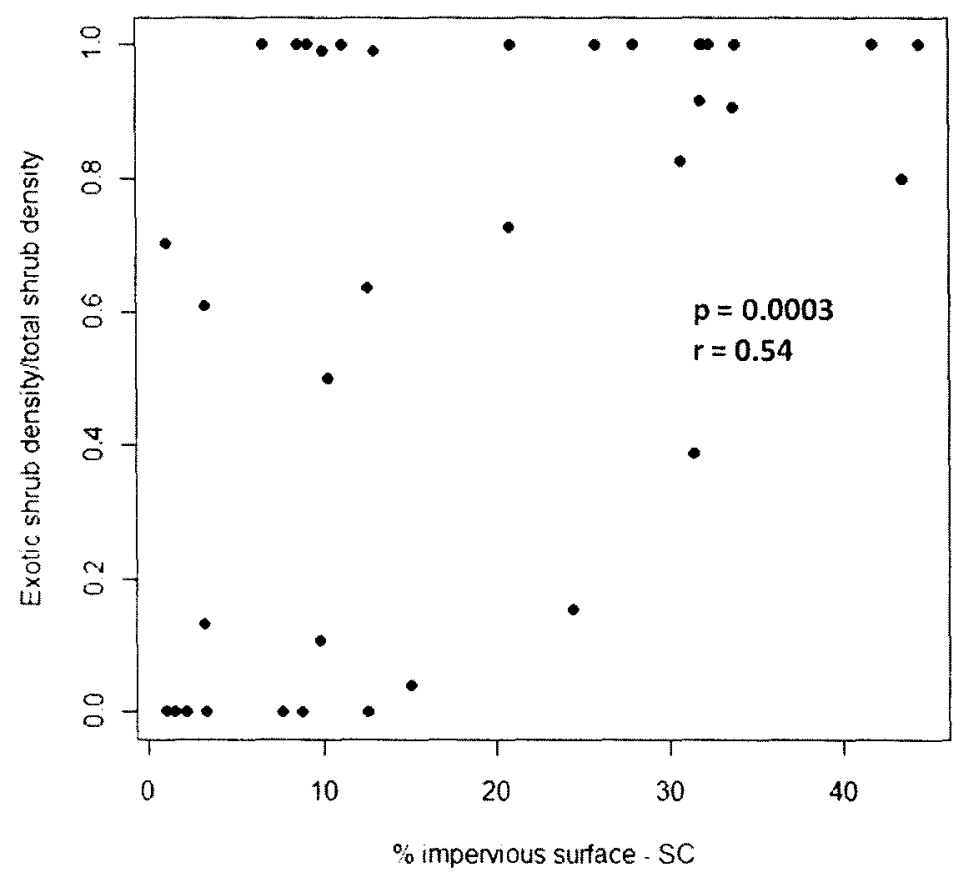

(b)

Figure 2-9a-b: Exotic shrub species richness (a) and density (b) proportions with increasing impervious surface (subcatchment scale). 


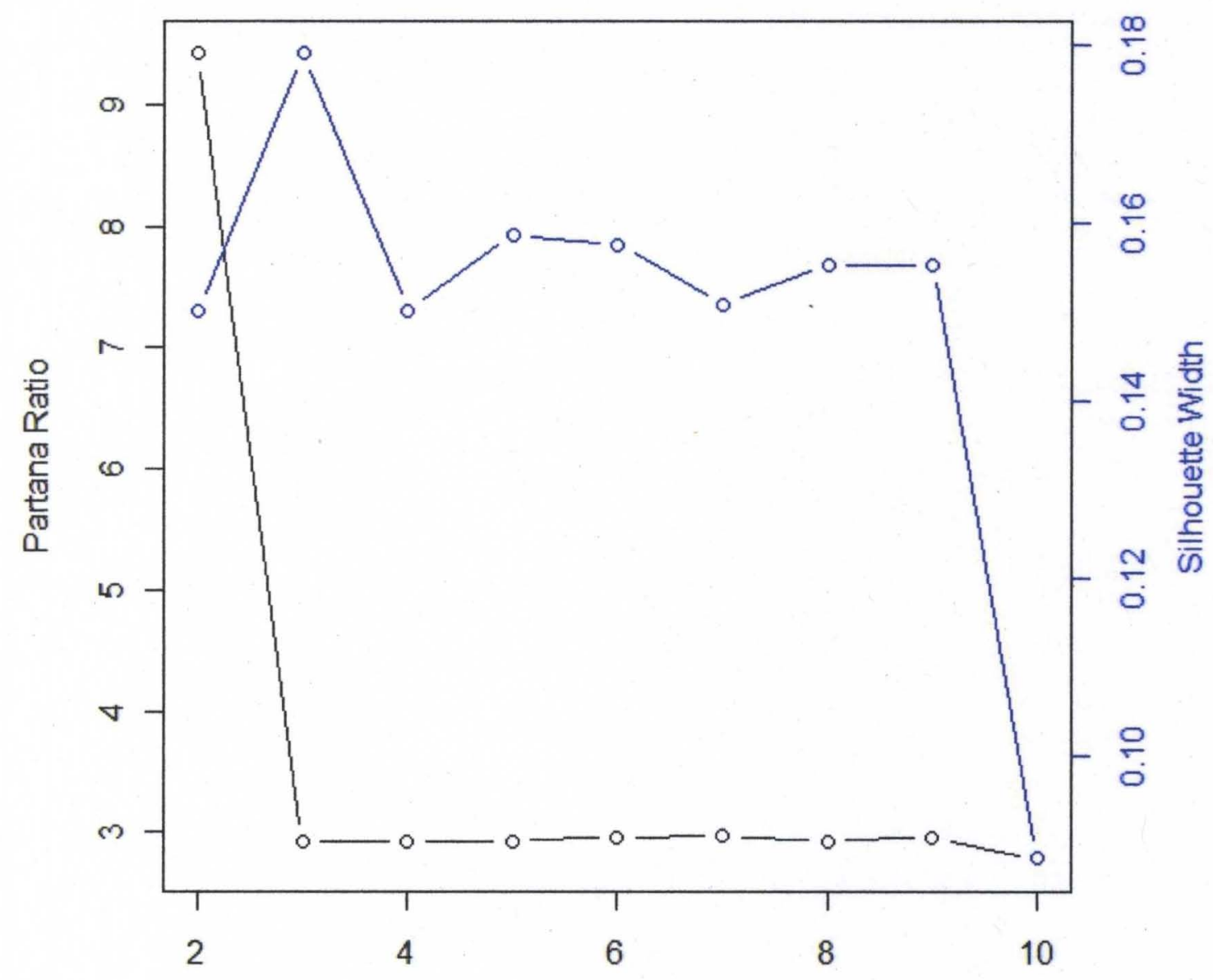

Number of Clusters

Figure 2-10: Partana ratios and overall silhouette widths for 2 to 10 clusters using optimal partioning. The 2-cluster configuration, while giving the highest silhouette width and partana ratio, results in two clusters, with one cluster containing all but one of the sites, and the other containing one site. I chose to group the sites into three and five clusters since those two options gave the highest values for overall silhouette width $(0.18$ and 0.16, respectively). Explanations of silhouette width given in Methods. 


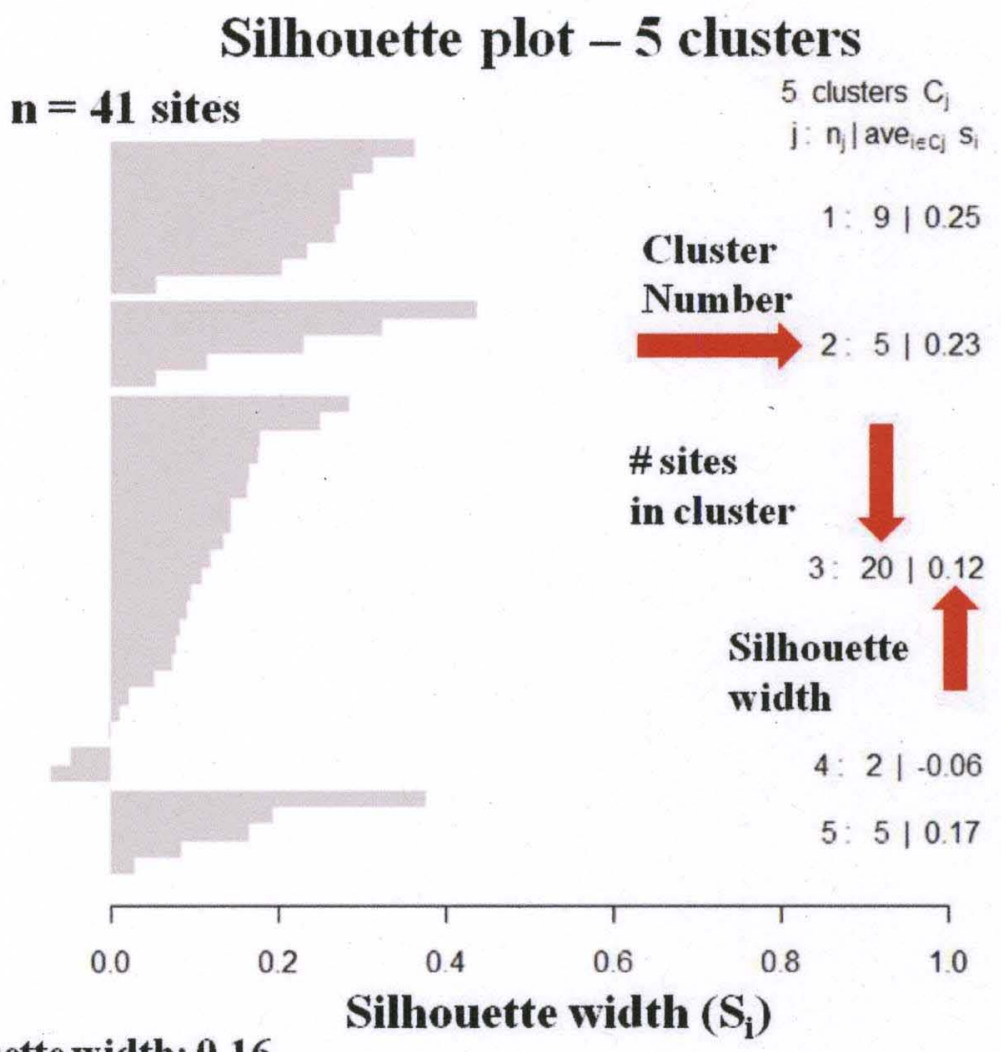

Average silhouette width: 0.16

Figure 2-11: Silhouette plot of cluster analysis using optimal partitioning and BrayCurtis distance resulting in five groupings of research sites. Sites within clusters are represented by gray bars, the length of each demonstrating the silhouette width (x-axis) of that particular site within the cluster. Cluster numbers, number of sites within the cluster, and mean cluster widths are to the right of each cluster. Average silhouette width, located below the numerical axis, is the mean of all silhouette widths for each site. Explanation of values given in Methods under the heading 'Data Analysis'. 


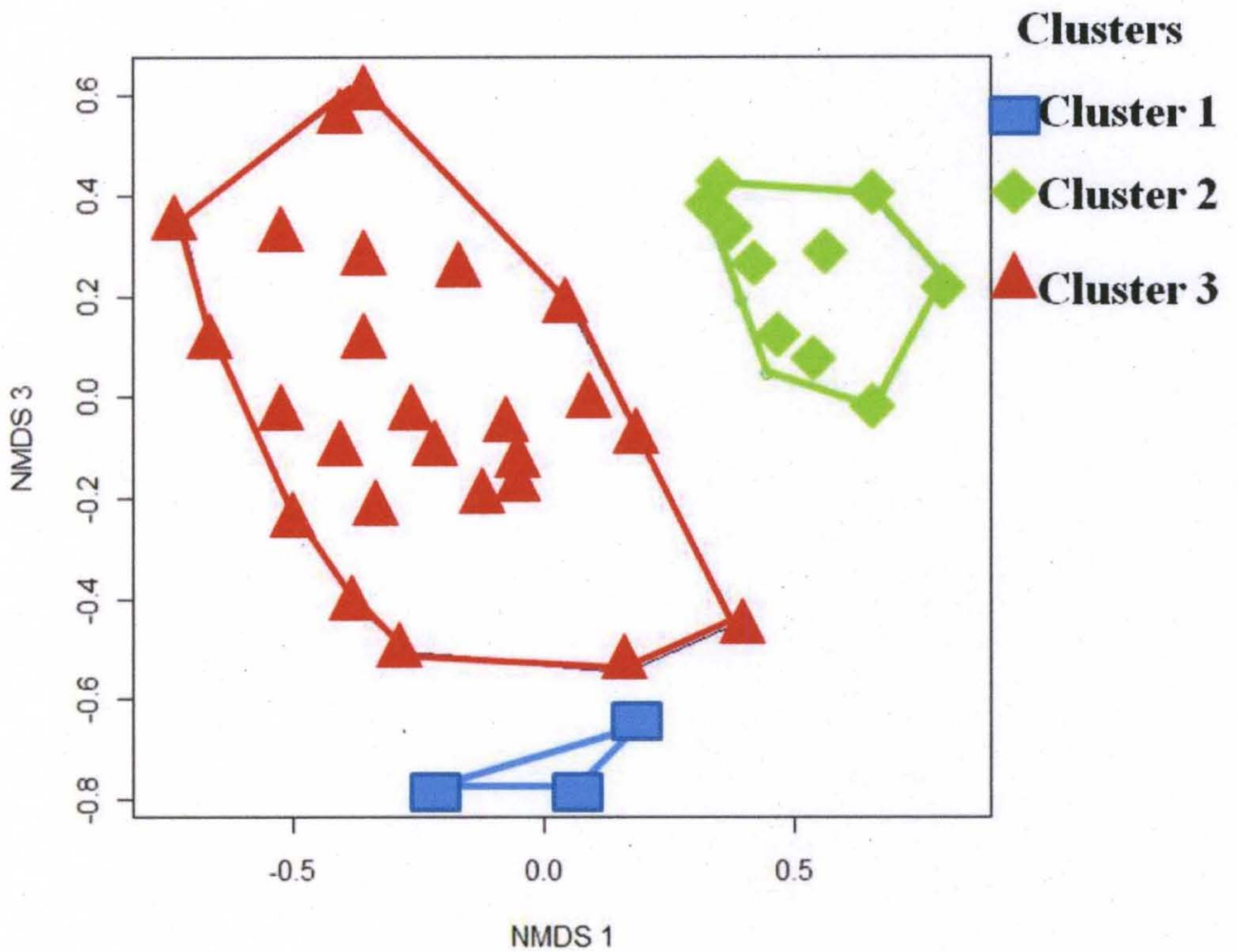

Figure 2-12: 3-dimensional configuration (only 2 dimensions shown) of woody plant data using non-metric multidimensional scaling (NMS) with sites (points) assigned to their respective groupings from the 3-cluster configuration from cluster analysis. Grouped clusters are shown along axes $1 \& 3$. No site overlap occurred in these three groupings in 3-dimensions indicating satisfactory agreement between the cluster analysis and the NMS solution. Plots in Cluster-1 (blue) are associated with the native shrub, Symphoricarpos orbiculata as its indicator species (Table 2-11), Cluster-2 in green is associated with the native shrub, Lindera benzoin, and the largest Cluster-3 (red) is associated with the exotic shrub, Lonicera maackii. 


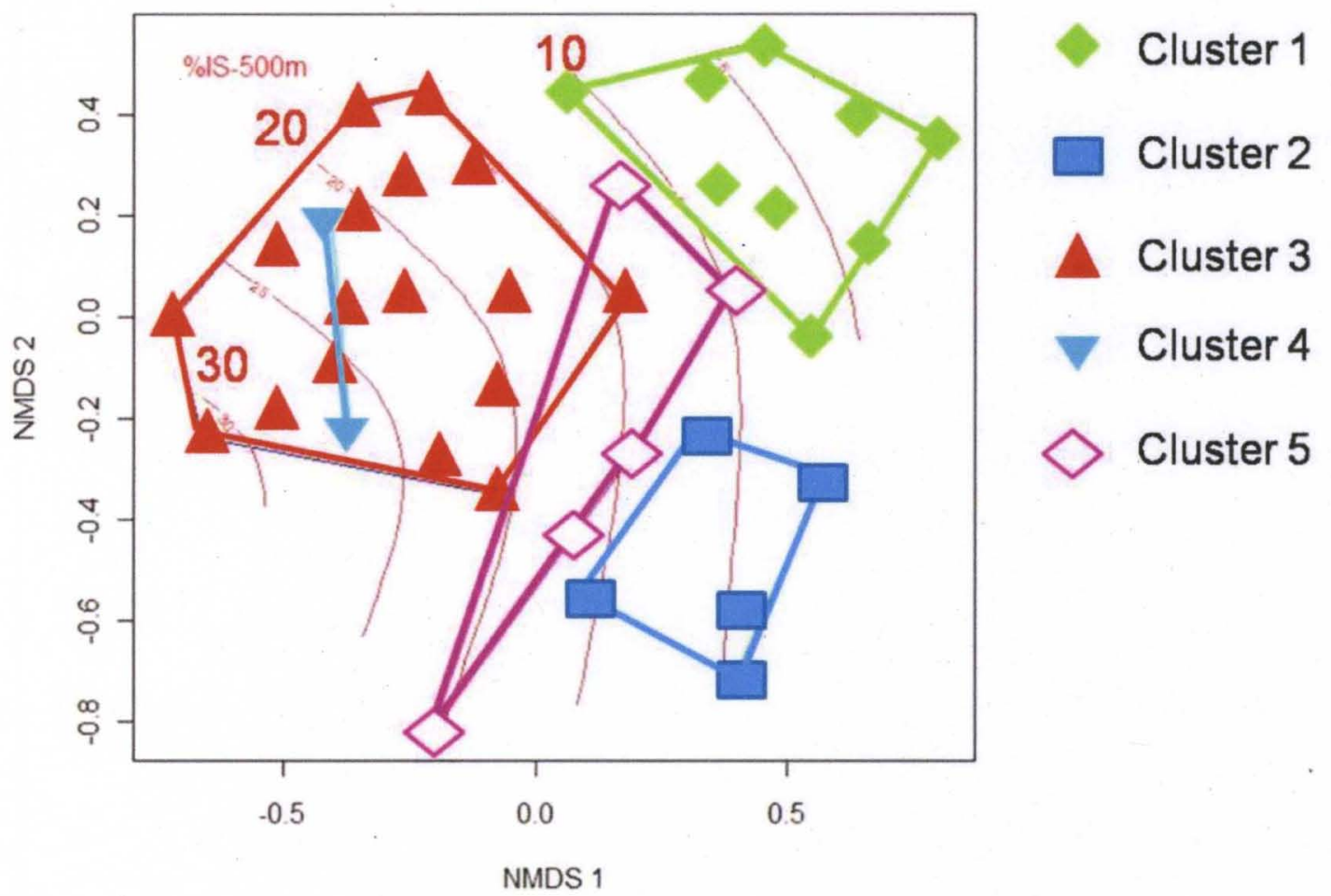

Figure 2-13: 3-dimensional configuration (only 2 dimensions shown) of woody plant data using non-metric multidimensional scaling coupled with cluster assignments from the 5-cluster configuration from cluster analysis. Isobar lines representing proportion of impervious surface within a 500-m radius of the research sites are presented to show the relationship to the clusters. Cluster-1 (green) is associated with the native shrub, Lindera benzoin, as a primary indicator species (Table 2-12), Cluster 2 (dark blue) with the native tree, Acer negundo, as its primary indicator species, Cluster 3 (red) with the exotic shrub, Lonicera maackii, as its primary indicator species, Cluster 4 (light blue) with anomalous outlier plots, and Cluster-5 (purple) with Acer negundo seedlings as its primary indicator species. 


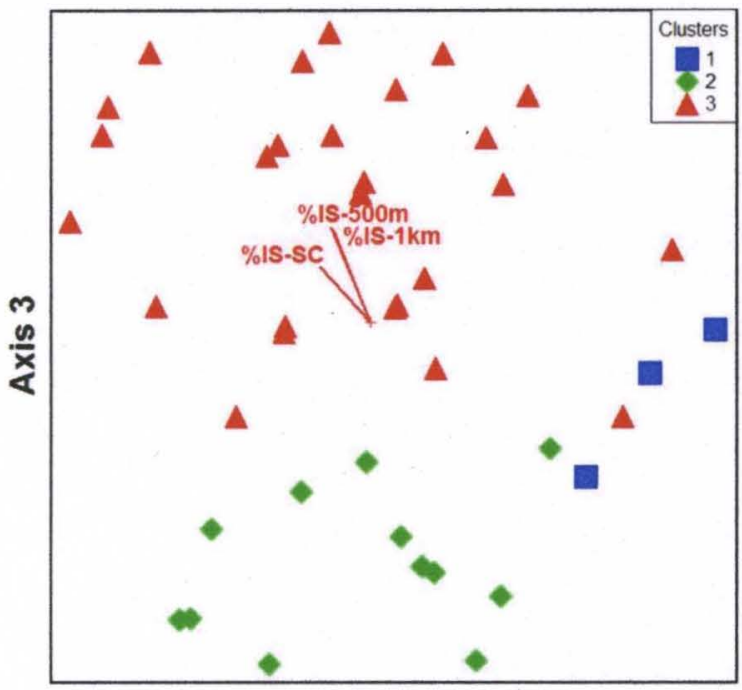

Axis 1

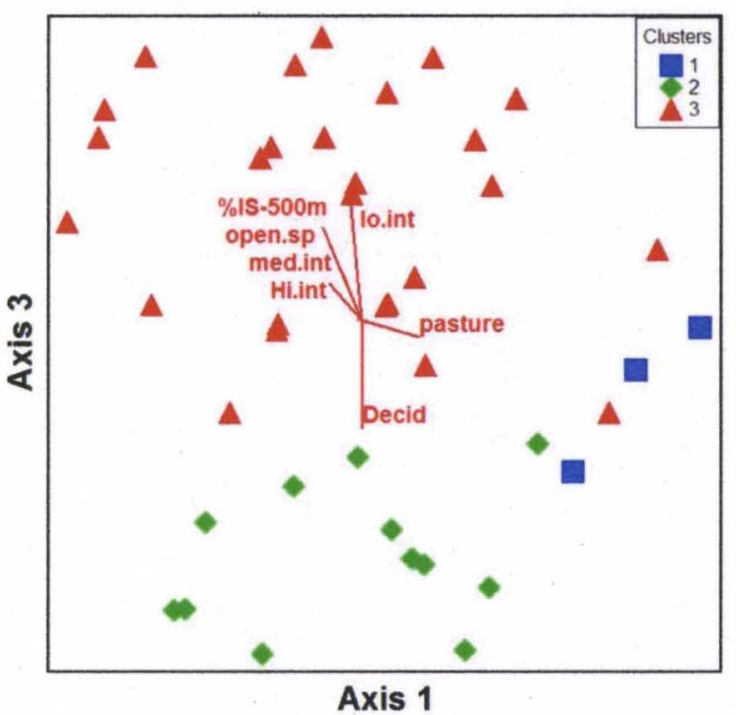

(a)

(b)

Figure 2-14a-b: Non-metric multidimensional scaling of woody plant data coupled with cluster assignments from the 3-cluster configuration using cluster analysis, shown as colored shapes. Clusters- 1 and -2 contain native shrubs as indicator species, while Cluster-3 contains an exotic shrub as an indicator species. Vectors representing site environmental metrics are pointing in the direction of increasing proportions, with the length of each vector representing the strength of correlation. Vectors for environmental metrics are shown only if they have an $r^{2} \geq 0.15$ along at least one axis. Environmental metrics shown along (a) axes $1 \& 3$ are the proportion of impervious surface (IS) surrounding each site at 3 scales: subcatchment (SC), 1-km and 500-m radius. Relationships of clusters with other environmental metrics are shown along the same axes in (b) and include low- medium- (med.int), and high-intensity (Hi.int) development, open-space developed (open.sp), \% IS within a 500-m site radius (\%IS-500m), pasture, and deciduous forest (Decid). Definitions are included in Appendix 3. Compare with Table 2-11 to get dominant and indicator species of the clusters. Explained variance $\left(\mathrm{r}^{2}\right)$ along each axis is as follows: Axis $1-0.14$, Axis 2 (not shown) -0.20 , Axis 3-0.34. 


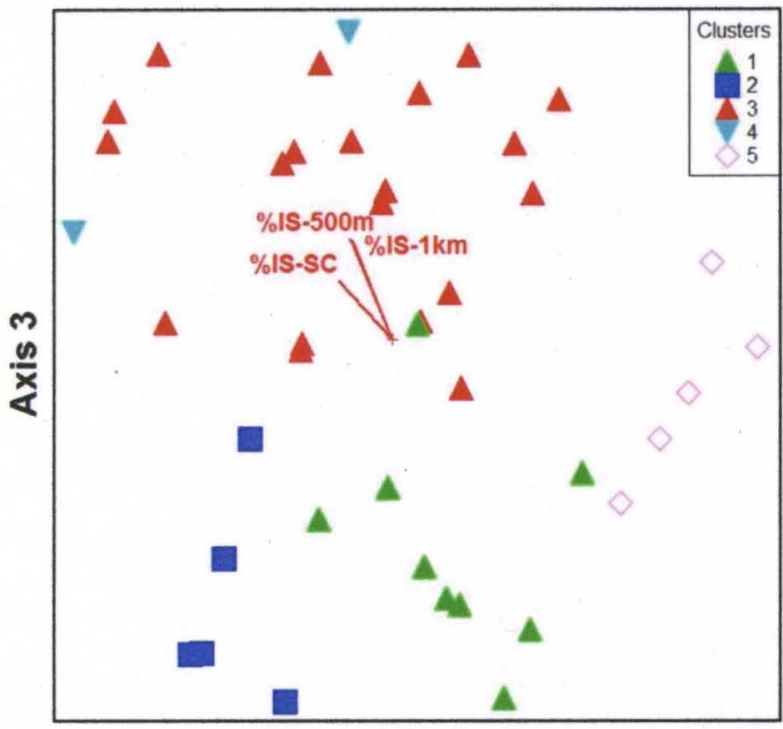

Axis 1

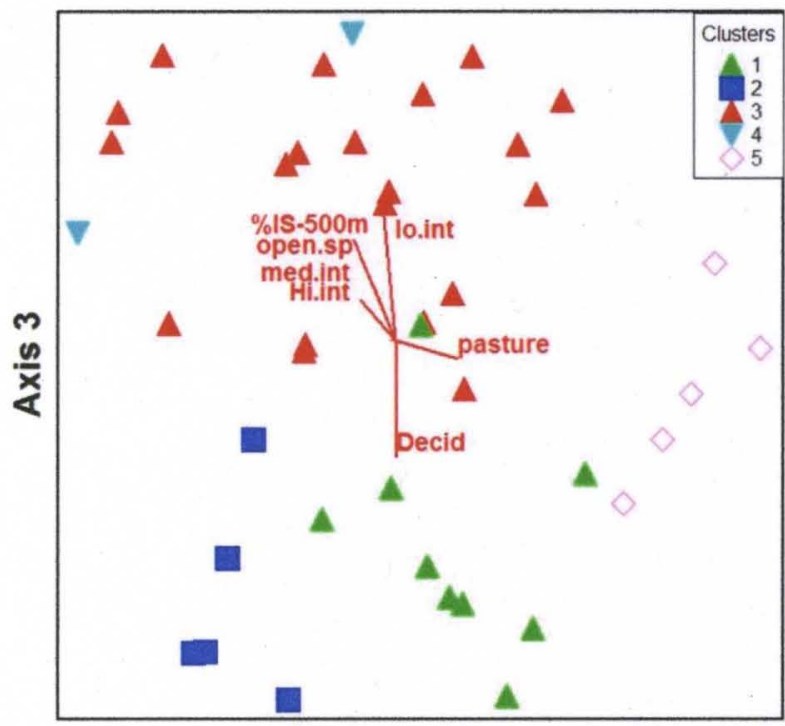

Axis 1 (a)

(b)

Figure 2-15a-b: Non-metric multidimensional scaling of woody plant data coupled with cluster assignments from the 5-cluster configuration using cluster analysis. Vectors representing site environmental metrics are pointing in the direction of increasing proportions, with the length of each vector representing the strength of correlation. Vectors for environmental metrics are shown only if they have an $\mathrm{r}^{2} \geq 0.15$ along at least one axis. Environmental metrics shown along (a) axes $1 \& 3$ are proportion impervious surface (IS) surrounding each site at 3 scales: subcatchment (SC), 1-km and 500-m radius. Environmental metrics shown along the same axes in (b) include low- (lo.int), medium- (med.int), and high-intensity (Hi.int) developed, open-space developed (open.sp), \% IS within a 500-m site radius (\%IS-500m), pasture, and deciduous forest (Decid). Definitions are included in Table 13. Compare with Table 2-12 to get dominant and indicator species of the clusters. Explained variance $\left(\mathrm{r}^{2}\right)$ along each axis is as follows: Axis $1-0.14$, Axis 2 (not shown) - 0.20, Axis 3-0.34 


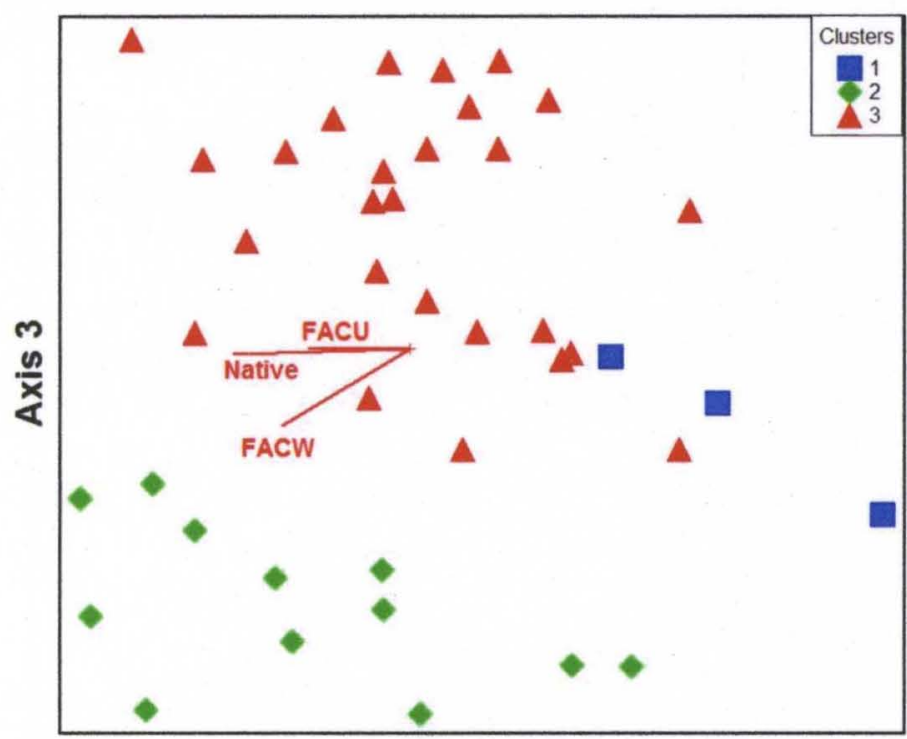

Axis 2

(a)

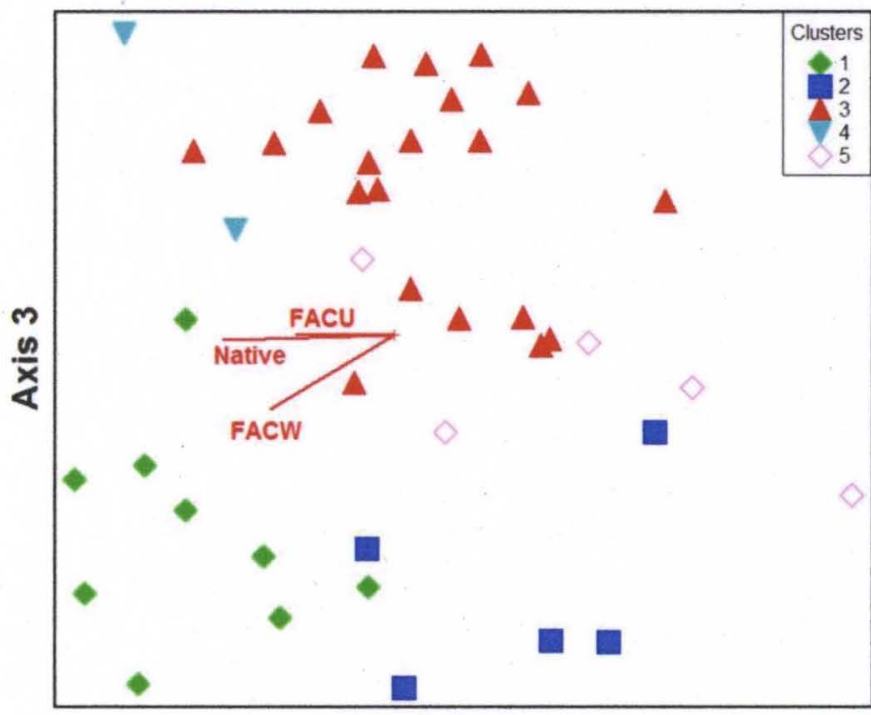

Axis 2

(b)

Figure 2-16a-b: Non-metric multidimensional scaling of research sites arranged according to woody species composition. Sites are color coded according to their cluster assignments from 3- and 5-cluster configurations using cluster analysis. Vectors representing species attributes are pointing in the direction of increasing importance values, with the length of each vector representing the strength of correlation. Vectors for species attributes are shown only if they have an $\mathrm{r}^{2} \geq 0.15$ along at least one axis. The 3cluster assignments are pictured in (a) and (b). The 5-cluster assignments are pictured in (c) and (d), with identical site configurations shown in (a) and (c), and (b) and (d). The species attributes shown are facultative-upland (FACU), facultative wetland (FACW), and native species $(\mathrm{N})$. Definitions and the full list of attributes included in the analysis are located in Table 14. Compare with Tables 8 and 9 to get dominant and indicator species of the clusters. Explained variance $\left(r^{2}\right)$ along each axis is as follows: Axis 1 (not shown) - 0.14, Axis 2-0.20, Axis 3-0.34. 


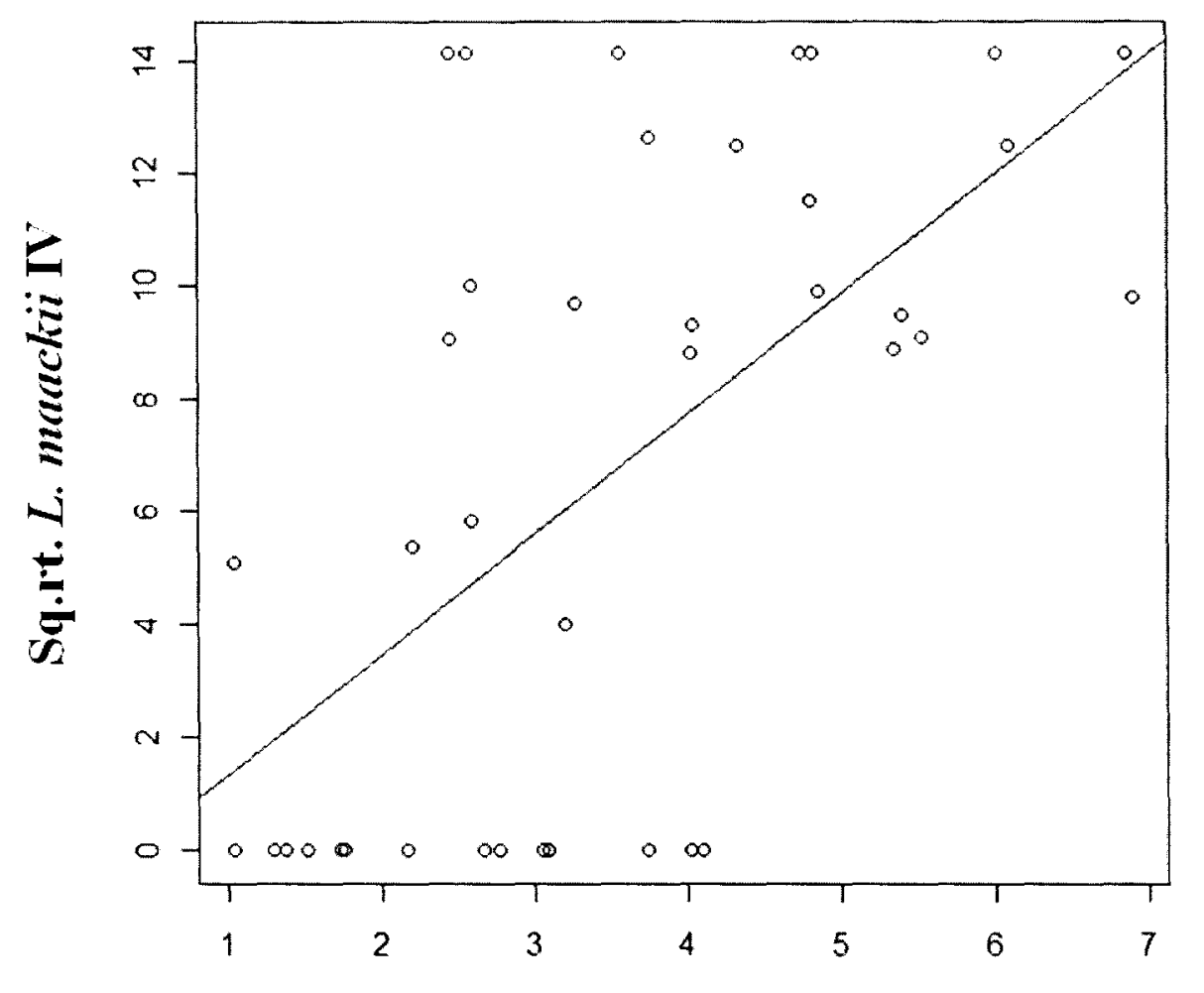

Sq.rt. \%IS (500m site radius)

Figure 2-17: Linear regression of $L$. maackii relative importance value (IV) against proportion of impervious surface within a radius of $500-\mathrm{m}$ of site centers. L. maackii IV significantly increased in relation to increasing proportions of impervious surface $(\mathrm{p}=$ 0.000057 ; adj. $\left.r^{2}=0.34\right)$. Data were square root transformed prior to regression. Regression of $L$. maackii densities against \%IS within a $500-\mathrm{m}$ site radius resulted in a lower coefficient of determination and $p$-value $\left(p=0.0002\right.$, adj. $\left.r^{2}=0.28\right)$ so the data are not shown. 


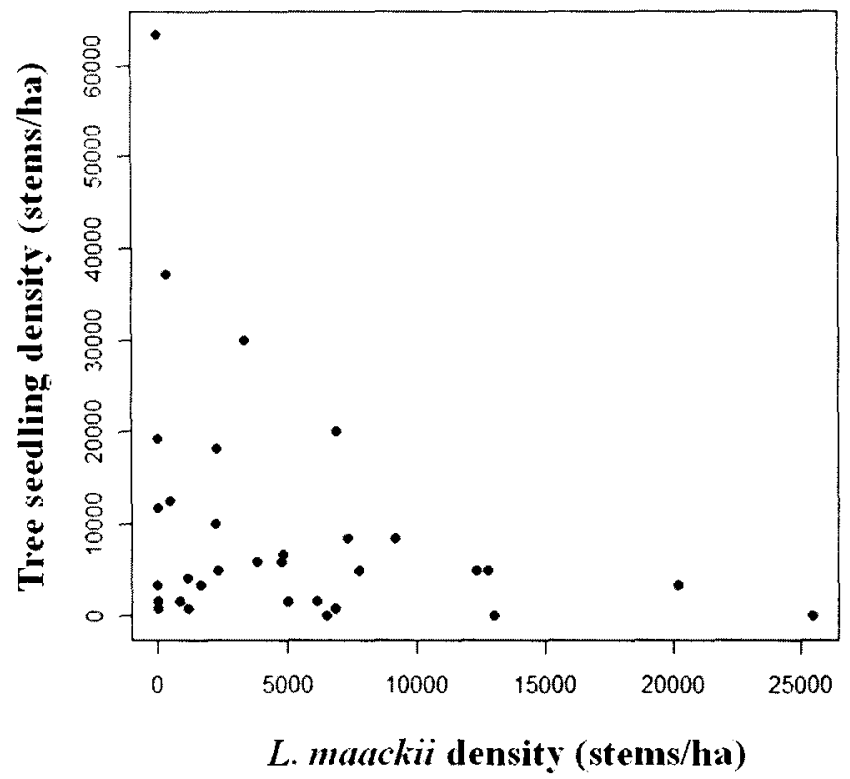

(a)

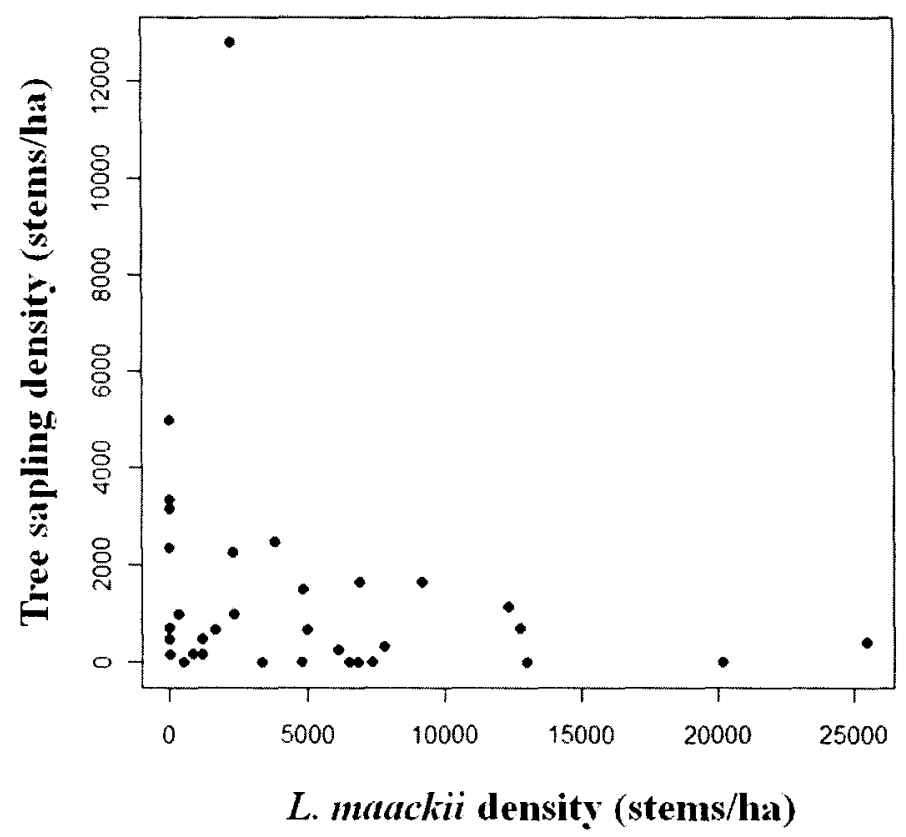

(b)

Figure 2-18a-b: Regressions of tree seedling and sapling densities compared to Lonicera maackii density at each site. Tree seedling density (a) and, to a lesser extent, tree sapling density (b) decreased in relation to increasing densities of $L$. maackii. The highest seedling and sapling densities occur at the lowest densities ( $<5000$ to $10000 \mathrm{stems} / \mathrm{ha}$ ) of L. maackil, and the lowest seedling and sapling densites at $L$. maackii concentrations $>10000$ stems/ha. 


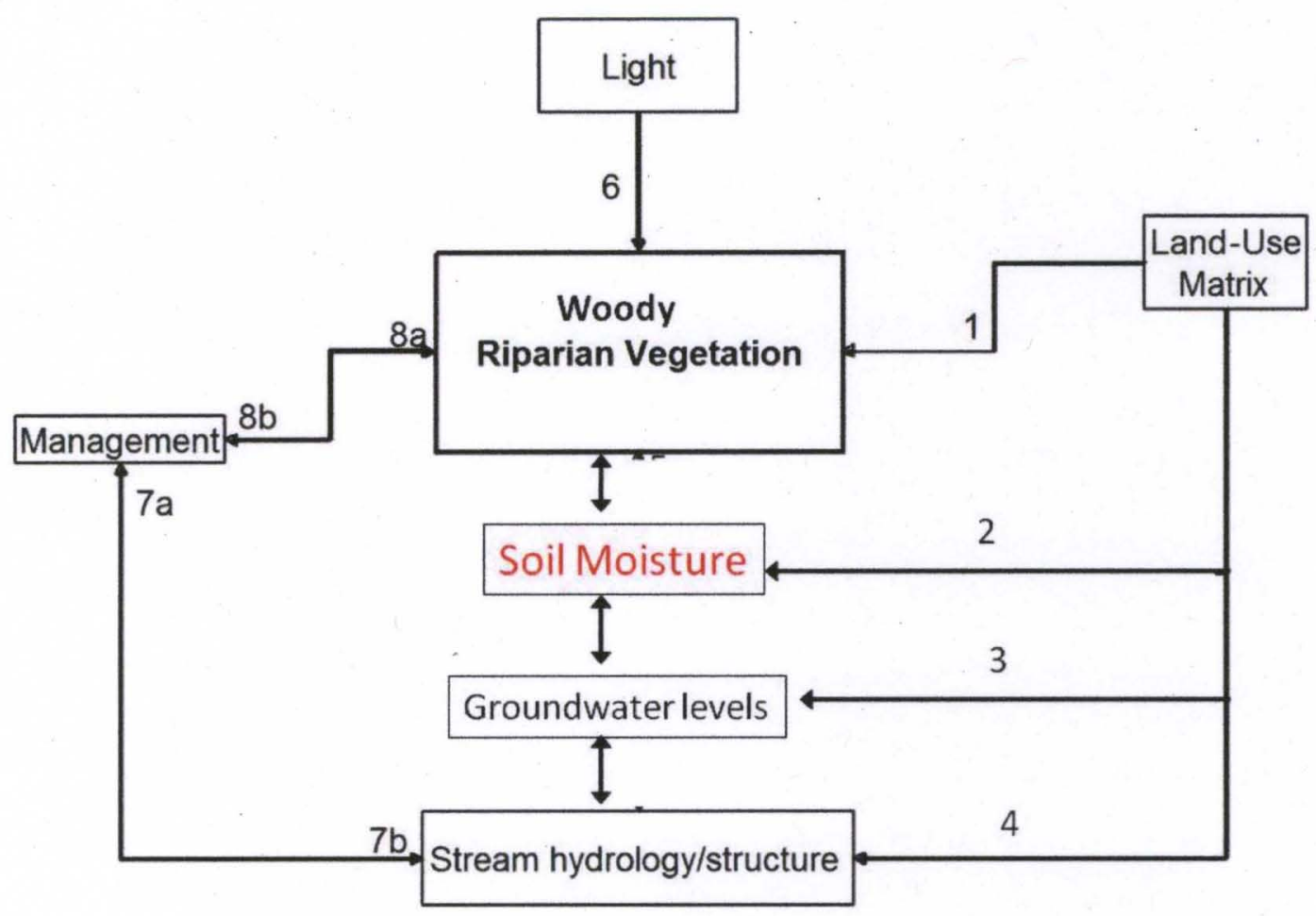

Figure 2-19: New conceptual model of variables that affect and are affected by vegetation within a riparian area modified from Figure 1-1. A direct link between groundwater levels and riparian vegetation was not found. However, since wetland species were found to a greater extent in rural riparian zones (Table 2-7, Fig. 2-16) soil moisture may be influencing plant species composition, although this would need to be verified in the field. 


\section{CHAPTER 3: VARIATION IN RIPARIAN GROUNDCOVER PLANT}

COMMUNITIES SURROUNDED BY URBAN, SUBURBAN, AND RURAL LAND-

\section{USE IN LOUISVILLE, KENTUCKY}

\section{Introduction}

Urbanization can affect the composition of riparian herbaceous and groundcover species at various scales. The existence of a greater number of exotics in the urban species pool creates the potential for their dominating riparian areas at landcape and regional scales. However, the plant communities realized in any location are highly influenced by site-level factors such as micro-scale soil nutrient, light and moisture differences (Guillaume 2002). As such, urban influences, such as nutrient deposition, on these species are likely to occur within a smaller site radius than for woody vegetation, which is more greatly influenced by macro-scale influences, such as forest management (Guillaume 2002). Localized urban effects, such as edge creation through reduction in size of woody riparian buffers, will likely influence groundcover (herb and vine) species composition and vulnerability to exotic species invasion. For example, Canadian riparian zones had greater numbers of exotic annuals at the urban end of an urban-to-rural gradient, while more perennial natives occurred primarily at the rural end (Moffatt and McLachlan 2004).

The interaction of urbanization and variable physical properties of the riparian zone can also result in different attributes of the plant community. Plant diversity has been shown to vary with location within riparian zones. Changing conditions of moisture 
and light availability with increasing lateral movement away from and longitudinal movement downstream have both been shown to relate to predictable changes in riparian plant diversity (Nilsson et al. 1994, Lite et al. 2005). For example, species richness of riparian herbaceous communities in arid environments in Arizona was found to decrease laterally away from the stream channel during a dry year (Lite et al. 2005). This finding was attributed to lower water availability farther from the stream, as the water table was found to drop with lateral distance from the channel. Furthermore, the opposite was true following monsoon rains and flooding, as herbaceous species richness increased with distance from the stream channel following increases in the water table. How plant species diversity changes with lateral distance from streams flowing through varying land-uses is less well understood.

In addition, riparian plant diversity has been shown to vary with catchment area and stream order. Nilsson et al. (1994) showed that plant species richness along a river in Sweden was higher along the main channel than its tributaries. One possible explanation offered was variation in mean annual stream discharge and peat and silt cover along streambanks. Lite et al. (2005) found a pattern of increasing mean species richness in an upstream direction following summer monsoon and flooding events in an arid environment, as herbaceous annual species rapidly responded to removal of resource limitation upstream. Whether such patterns exist for riparian herbaceous communities in response to changing resource availabilities across an urban-to-rural land-use gradient has yet to be determined.

The overarching goal of this project was to determine how groundcover plant communities (herbs and woody vines growing over the soil) along streams vary with 
degree of urban and suburban development in three watersheds in Jefferson and Oldham counties in Kentucky. The first objective was to determine whether potential relationships existed between riparian groundcover vegetation assemblages and impervious surface cover and NLCD land-cover types at different spatial scales. Specific predictions associated with this objective were that distinct groundcover vegetation communities exist that can be partially correlated with impervious surface cover, used as an index of urbanization. The second prediction was that the sphere of influence of different land covers would correlate more strongly with differences in groundcover vegetation with decreasing site radius, given the sensitivity of herbaceous vegetation to microscale as opposed to macroscale environmental variation (Guillaume 2002).

The second objective was to determine whether differences in plant species diversity existed along this urban-to-rural land-use gradient as well as with increasing catchment area and lateral distance from the streambank. There are three predictions associated with this objective, the first being that groundcover plant diversity along this gradient would be higher in suburban riparian areas than in either urban or rural riparian areas for two reasons: 1) because of the likely greater availability of diverse seed from residential areas than in rural locations (McKinney 2008), and 2) because of the prevalence in urban riparian areas of Lonicera maackii, an invasive shrub known to reduce herb layer diversity (Collier et al. 2002, Loewenstein and Loewenstein 2005) (see Chapter 2). The second prediction was that species richness would increase with total upstream catchment area, since other studies have shown that plant species richness increases with downstream distance (Bendix 1997, Bendix and Hupp 2000, Lite et al. 2005). The third prediction was that groundcover plant species richness would increase 
with lateral distance from the stream due to the decreasing influence of floodwaters farther from the stream (Lite et al. 2005). To my knowledge, this is the first study to have compared herb and woody vine species richness in the ground layer of riparian environments across different land-use types and with lateral distance from the stream.

The third objective was to determine the extent to which native and non-native groundcover vegetation was associated with varying land-cover types. Since one consequence of urbanization is creation of edges, I predicted that native vegetation would be more dominant in riparian assemblages associated with greater proportions of forested land-cover types, and exotic vegetation would take on greater importance in riparian assemblages associated with urban land-cover types. The fourth objective was to determine the extent to which wetland and upland species were associated with various land-cover types. Because of impervious runoff, stream incision due to greater impervious cover, and the urban heat island effect, I proposed that higher streambanks would be found in urban areas and that this trend would be correlated with groundcover species composition. I specifically predicted that obligate and facultative wetland species would assume greater dominance in riparian assemblages associated with forested rural land-cover types and that facultative and upland species would exhibit greater dominance in riparian assemblages associated with urban land-cover types. The fifth objective was to determine groundcover species associations with and diversity within plots dominated by the shrub, Lonicera maackii. Since L. maackii is an exotic species with allelopathic properties and strong shading capabilities, I predicted that herb layer species composition would differ greatly and that species richness and other measures of diversity would be lower under this shrub than where this shrub was less dominant. 


\section{Methods}

Study Area

The study area consisted of three individual watersheds located within and adjacent to Louisville, Kentucky, USA (Latitude $38^{\circ} 15^{\prime} \mathrm{N}$, Longitude $85^{\circ} 46^{\prime} \mathrm{W}$ ), which has a population of approximately 700,000 with a mean density of 695 people $\mathrm{km}^{-2}$ (U.S. Census Bureau 2008). These watersheds, Beargrass Creek (Middle Fork), Goose Creek, and Harrods Creek (South Fork), lie adjacent to each other with all streams flowing in a primarily westerly direction and eventually emptying into the Ohio River (Fig. 3-1).

The three watersheds are of approximately equivalent sizes, containing varying proportions of impervious surface cover (ISC; Table 3-1). Beargrass Creek watershed, located in the north-northeast portion of Jefferson County, $\mathrm{KY}$, is approximately $65 \mathrm{~km}^{2}$ (Metropolitan Sewer District 1999b) and contains the greatest proportion of impervious surface cover (33\% ISC). This Middle Fork of Beargrass Creek joins the South Fork before flowing into the Ohio River. Several stretches of this stream are featured on Kentucky's Division of Water 303d list, which list streams with impairment issues as well as the pollutant(s) causing the impairment (Kentucky Environmental and Public Protection Cabinet 2008). Since combined sewer overflows (CSOs) commonly occur in this stream, the presence of fecal coliform and sewage are stated as the primary problem pollutants within Beargrass Creek.

Goose Creek is a $50-\mathrm{km}^{2}$ watershed also in Jefferson County, in-between and adjacent to Beargrass and Harrods Creek watersheds (Metropolitan Sewer District 1999a). Of the three watersheds in this study, impervious surface cover is intermediate (20\% ISC). The creek is composed of a pair of forks that meet before emptying into the 
Ohio River. Goose Creek is also listed on the $303 \mathrm{~d}$ list, with cadmium and fecal coliform levels stated as impairment issues.

South Fork Harrods Creek watershed $\left(60-\mathrm{km}^{2}\right)$, which has the lowest impervious surface cover of the three ( $10 \%$ ISC), is located primarily in neighboring Oldham County, but extends into northeast Jefferson County. South Fork Harrods Creek eventually joins North Fork Harrods Creek to become Harrods Creek before flowing into the Ohio River. Sections of the larger Harrods Creek network made the $303 \mathrm{~d}$ list in years past, specifically the portion passing through Jefferson County which is a more developed area. As in the other two watersheds, fecal coliform has traditionally been a problem (Metropolitan Sewer District 1999c). The current 303d list contains Harrods Creek with fecal coliform as an impairment issue, although this information is for the stream section downstream of where the North Fork and the South Fork Harrods Creek join.

Watershed classification

Watersheds were chosen from digitized catchment layers downloaded from the Louisville/Jefferson Information Consortium (LOJIC) database (LOJIC 2010). LOJIC is a multi-agency consortium that maintains a geographic information systems (GIS) database to serve primarily Jefferson County, KY, although limited information is available for a portion of Oldham County, KY. After checking for topographical accuracy, I divided catchments into subcatchments based on stream order and topography from the original LOJIC layers. I further divided subcatchments of higher stream orders with longer reaches into two or three subcatchments. Data layers from the LOJIC 
database were clipped to each of these subcatchments. Beargrass Creek watershed contained thirteen subcatchments, Goose Creek watershed contained twelve subcatchments, and Harrods Creek watershed contained fourteen subcatchments.

Impervious land-cover classification was determined using three data layers from the LOJIC database digitized in 2001. These layers included buildings, miscellaneous transportation, and roads. The buildings layer included all built structures, whether commercial or residential. The roads layer included all paved road surfaces, excluding parking lots. The miscellaneous transportation layer included secondary transportation features such as driveways, parking lots, and sidewalks. The area of land covered by impervious surfaces for each subcatchment was determined using ArcGIS 9 (ESRI 2010) and was divided by the total land area for each subcatchment to determine the proportion of impervious surfaces covering the landscape.

Categorization of subcatchments into urban, suburban, and rural land-use categories was modified from Schuler (1994). For this study, urban subcatchments were defined as containing $\geq 30 \%$ impervious surface cover. Rural subcatchments contained $\leq 10 \%$ impervious surface cover and suburban subcatchments contained between $10 \%$ and $30 \%$ impervious surface cover. To explore the degree of association between riparian plant communities and proximity of different types of land use and cover, research sites were classified by land use based on impervious surface cover at three scales. The first and largest areal scale was the subcatchment level, where sites were classified as urban, suburban, or rural based on the proportion of impervious cover in the subcatchment in which they were located. Impervious surface cover was calculated at two additional and smaller scales surrounding the sites. These site buffers at a radius of $1-\mathrm{km}$ and $500-\mathrm{m}$ 
from the site center were created using ArcGIS 9. The proportion of impervious surface cover was determined for these buffers in the same way as for the subcatchments. Sites were then classified into one of the three land-use categories in the same way as the subcatchment. Urban, suburban, rural categorization of sites was changed for each relevant analysis depending on the scale at which impervious cover was calculated.

\section{Site selection and plot establishment}

Forty-one riparian sites were chosen from within the aforementioned 39 catchments. Riparian zones were defined by topography and structural confinements. Areas of level ground between the streambank and either an upland slope, structural barrier (roads, parking lots, buildings), or other obstruction (grazed pasture, ephemeral stream channel) were considered as being within the riparian zone. Sites were chosen randomly using ArcGIS 9 as follows. A vertical measurement line with units in meters was drawn the length of the stream section within the subcatchment. A random number multiplied by 100 meters was used to find the location of the site along the vertical measurement line. Sites were visited and chosen if they were at least partially covered with unmanaged vegetation and at least 100 meters from the nearest fork to avoid sampling dual riparian zones from separate channels. Riparian zones located completely within regularly used pastures and those found to be mown to the edge of the streambank were not used for this study. Only one side of the stream was sampled at each site. If both sides contained vegetation, the larger side was chosen if it fit the criteria above, if it was reachable, and if the proper permissions could be attained. If only one side of the stream contained vegetation then that side was chosen. Locations where at least one full plot 
could not be placed (riparian width $<12-\mathrm{m}$ ) were sampled with a partial plot due to the time and expense of acquiring permission to access sites, and to avoid potential biases of purposefully choosing a site of a particular width. The distribution of riparian widths by stream order and land-use category for the sites chosen is presented in Table 3-2.

After visiting the locations identified by ArcGIS selection, the location of the actual study site was chosen by taking a randomly determined number of steps to establish the center of a transect. I used a compass to extend a transect tape perpendicular to and away from the stream channel up to 91 meters, if possible. Circular plot centers (plot radius $=5.64-\mathrm{m})$ were established beginning at 6,46 , and 86 meters away from and perpendicular to the stream channel. Three belt transects measuring $10 \times 2$ meters were nested within the circular plots, with the long edge perpendicular to the stream. The distance between midlines of adjacent belt transects was four meters. Twelve 1 x 1 meter quadrats were nested within the belt transects (four quadrats per belt transect). Quadrats were placed every two meters down the midline of each belt transect, so that the centers of adjacent quadrats within a belt transect were three meters apart (Fig. 3-2).

The circular plots were used in a previous study to estimate the abundance and species identity of trees. Belt transects were used to measure shrub live stem densities. Percent cover and identity of groundcover species (herbaceous and woody vine species), as well as number and percent cover of $L$. maackii seedlings, were determined in each $1 \mathrm{x}$ 1 meter quadrat. For all vegetation data, only livings plants were considered. L. maackii sampling was conducted in 2005 (June to August) and in 2006 (May to October). Groundcover plant data were collected from May to October, 2006. 
Plant identification and classification

Plant identifications were made in the field whenever possible. The primary source used for identification of shrubs and vines was Wharton and Barbour (1973). Plants that could not be identified in the field were collected for later keying using Jones (2005). Collections included as many parts of the plant as possible, including stem, leaf, and flowering parts. However, the authority for all species names used here was the USDA PLANTS Database (2010). Species provenance at the continental level was determined using Jones (2005) and the USDA PLANTS Database (2010). Two categories of species provenance at the continental scale were used: native and exotic. Term usage is as defined in the USDA Natural Resources Conservation Service (2009). Native plants are those that have developed over hundreds of years in an area (in this instance, the continental U.S.) and were present prior to European settlement. Exotic plants are defined herein as those introduced with human assistance to a continent on which they were not previously found prior to European settlement.

\section{Importance value and diversity}

For each site (one to three plots), an importance value (IV) was calculated for each groundcover species as the sum of average percent cover and frequency. Average importance values were calculated across quadrats at each site based on the arithmetic mean of the percent cover of each species. Percent frequency was determined for each species based on the number of quadrats in which a particular species was present divided by the total number of quadrats at each plot and multiplied by 100 . 
Eq. 1

Groundcover species IV $(\%)=$ Average $\%$ cover $+\%$ Frequency

Species richness (S), Shannon's diversity $\left(\mathrm{H}^{\prime}\right)$ and Simpson's reciprocal (1/D) indices, as well as Shannon's equitability index $\left(\mathrm{E}_{\mathrm{h}}\right)$ were computed for each site using data collected only from the plot nearest the stream (see Chapter 2 Methods for full equations and references). Since site and plot sizes varied, richness was calculated as S/Log(Area sampled) at the site scale (for up to three plots) and at the nearstream plot scale. Log transforms were used on richness data to normalize variance. Two sites with the lowest richness were eliminated from site and nearstream analyses to normalize residuals and stabilize variance. Data from only nearstream plots were compared using Shannon's, Simpson's, and equitability diversity analyses when examining potential relationships between plant community diversity and land cover and use. Average $\%$ cover for each species was computed for each nearstream plot and multiplied by 100 to obtain all integer values. Shannon's equitability index $\left(\mathrm{E}_{\mathrm{h}}\right)$ was computed as a measure of species evenness as $E_{h}=H / \ln (S)$, where $H^{\prime}$ is Shannon's diversity index and $S$ is species richness.

Community diversity was expressed at each site using species richness, Shannon's Index and Simpson's Reciprocal Index. In addition to nearstream plot richness, site richness was calculated by totaling species across all plots for a site. Because of different riparian widths and plot sizes, richness was calculated for all sites as well as nearstream plots as the number of species divided by the log of the area sampled (Conner and McCoy 1979, Nilsson et al. 1997). Average percent cover of groundcover 
species (herbaceous species and vines) was used to compute Simpson's reciprocal (1/D)

(Simpson 1949, Magurran 2004) and Shannon's (H') (Shannon 1948, Magurran 2004)

indexes for plots nearest the stream. EstimateS (Colwell 2009)was used for computing

diversity indices. In EstimateS, diversity index estimators were not used, so indices were computed using the observed sample order (no randomization).

Metrics for wetland indicator status (WIS) and species provenance (NE;

$\mathrm{N}=$ native, $\mathrm{E}=$ =xotic) were computed for each site to assess correlations with various landcover variables. Species were labeled with their WIS from the USDA PLANTS database as well as NE as describe previously. WIS and NE codes and meanings are shown in Appendix 2. A numerical value was assigned to each site for each category of WIS and NE using the groundcover IV's calculated for each species in each site. Values were calculated by averaging the IV's of the species in each WIS and NE category and adding a weighting factor to account for the number of species at each site within that particular category. The weighting factor was the number of species within a particular category divided by the total number of species within the site. Being based upon absolute IV's, the maximum value for any species indicator category was 300 (maximum 200 for average absolute IV + maximum 100 for the weighting factor). Values were converted to percent form by multiplying by 100 .

Eq. 2

Native metric $=\left(\right.$ Native $\mathrm{Sp}_{1} \mathrm{IV}+$ Native $\mathrm{Sp}_{2} \mathrm{IV}+$ Native $\mathrm{Sp}_{3} \mathrm{IV}+\ldots \ldots .+$ Native $\mathrm{Sp}_{\mathrm{N}}$ IV)/N + Weighting factor [\# native spp./Total \# spp. ${ }^{*} 100$ ] 
Eq. 3

Exotic metric $=\left(\right.$ Exotic $\mathrm{Sp}_{1} \mathrm{IV}+$ Exotic $\mathrm{Sp}_{2} \mathrm{IV}+$ Exotic $\mathrm{Sp}_{3} \mathrm{IV}+\ldots \ldots+$

Exotic Sp $\mathrm{N}_{\mathrm{N}}$ )/N + Weighting factor [\# exotic spp./Total \# spp.*100]

The same form of equation was used to compute metrics for each category of WIS. These values were included in an environmental matrix and used to determine correlations with research sites arranged according to similar species composition in ordination space.

\section{Distance From Stream and Catchment Area Analyses}

Relationships were also explored between groundcover plant diversity and perpendicular distance from the stream by land-use type. Only nearstream plots were used, since all sites had at least this plot in common. At each nearstream plot, groundcover species richness was computed for each row ( $n=1$ to 4 rows per plot depending on plot size) of quadrats ( $\mathrm{n}=3$ per row). Quadrats were arranged as previously described, with rows parallel to the stream spaced every two meters beginning one meter from the streambank edge. The four rows were positioned from 1 to 2,4 to 5,7 to 8 , and 10 to 11 meters from the streambank. Richness values for the two rows nearest the stream were $\log$ transformed prior to analysis to meet assumptions of normality. Richness for the furthest two rows met assumptions of normality without data transformation. In some instances, mown lawns became the only vegetative cover beyond a certain lateral distance from the stream. In these cases the lawns were treated as monocultures and richness was assigned a value of 1 . 
Linear regression was used to compare groundcover richness for nearstream plots with cumulative upstream catchment area and bank height adjacent to the plot as a metric for depth to groundwater. Lawns were not included in determining richness values in these analyses, since lawns can be considered a type of land-use. Richness was then normalized to account for varying plot sizes as (richness/Log (area sampled)). Plots were also assigned to urban, suburban and rural land uses based on \% impervious surface at the subcatchment scale. For each land-use category, groundcover richness was regressed as a response variable against cumulative catchment area $\left(\mathrm{km}^{-2}\right)$ upstream of and including the subcatchment in which the plot was located, as well as against bank height $(\mathrm{cm})$ measured adjacent to the midline of each plot. Cumulative catchment area and groundcover richness were natural log transformed to better meet assumptions of the linear regression model.

\section{Honeysuckle analysis}

The relationship between L. maackii stem density and measures of groundcover diversity was assessed using linear regression. Again only nearstream plots were used in these analyses. In the case of exploring potential relationships between L. maackii density and percent cover of the vine Euonymus fortunei, two different approaches were used. In the first, plots were included in the analysis if they contained E. fortunei, but not necessarily $L$. maackii to demonstrate the abundance of $E$. fortunei in the presence and absence of $L$. maackii. Data were square root transformed prior to analysis, and one site was removed to normalize residuals and variance. In the second analysis, sites were included only if they contained both species occurring together in the same plot to 
demonstrate the degree to which the abundances of the species were related to each other. Data were log transformed and two sites were removed to normalize residuals and variance.

To explore relationships between groundcover species diversity and L. maackii and E. fortunei abundances, I chose sites where those two species occurred and regressed values for site diversity indices $\left(\mathrm{H}^{\prime}, 1 / \mathrm{D}, \mathrm{E}_{\mathrm{h}}, \mathrm{S}\right)$ against either $L$. maackii density or $E$. fortunei percent cover. In regressions involving L. maackii, stem densities of $L$. maackii and diversity index values (except $\mathrm{H}^{\prime}$ ) were log transformed, and two sites were excluded prior to analysis to normalize residuals and variance. In regressions with $E$. fortunei, the two sites with the lowest and highest $E_{h}$ were excluded from analysis to reduce heteroscedasticity. In addition, E. fortunei percent cover was square root transformed prior to analysis with 1/D and $\mathrm{H}^{\prime}$, and one site was removed prior to regressions against $\mathrm{H}^{\prime}$ to normalize residuals and variance.

\section{Environmental parameters}

Several environmental variables within and external to and the plots were used to explore their ability to explain the variation in plant species composition. More proximate site variables included bank height, canopy openness, and plot size. Factors that integrated influences from the greater landscape included \% impervious surface cover (at three scales: the subcatchment, within $0.5-\mathrm{km}$ radius and within a $1-\mathrm{km}$ radius of site center), land-cover categories, stream order, cumulative catchment area, and property value. 
Bank height was measured from the first terrace out of the channel and referenced to the depth at middle of the stream bed. Where the center of the channel was not reachable from the bank or by wading, bank height was measured at arm's reach. Bank height was measured once at the midline of each site $(n=41)$. These measurements were used as a proxy for depth to groundwater in order to determine correlations with species composition at the sites. Additional measurements were taken 50 meters to either side at thirteen of the sites where future studies of soil respiration were conducted. These thirteen additional measurements were averaged with the 41 site measurements to assess differences in overall bank height by land-use category.

Since canopy openness and hence light can also be a determinant of species presence and dominance, a spherical densiometer (Lemmon 1956, 1957), held over the middle of each of twelve $1-\mathrm{m}^{2}$ quadrats, was used to determine this measure. These values were averaged to obtain percent canopy openness for each site, as well as for each distance interval from the stream as described previously under the 'Distance Analysis' heading. Plot sizes were based on sampling areas of circular plots (Fig. 3-2) and ranged from a minimum of $28-\mathrm{m}^{2}$ to a maximum of $300-\mathrm{m}^{2}$. Thirteen sites out of 41 had an area less than $100-\mathrm{m}^{2}$, and seven sites had an area greater than $100-\mathrm{m}^{2}$ (one being $150-\mathrm{m}^{2}$, five being $200-\mathrm{m}^{2}$, and one site being $300-\mathrm{m}^{2}$ ). The remaining sites were $100-\mathrm{m}^{2}$.

Impervious surface cover was determined as described previously and the three scales were used to explore which scale better predicted plant community composition. Finer-grained land-cover categorizations (developed -open space, -low, -medium, and high intensity, deciduous forest, mixed forest, herbaceous, pasture, cultivated crops, and wetlands) were made using the U.S. Geological Survey's National Land Cover Database 
from 2001 (NLCD 2001; Appendix 3) acquired through LOJIC. NLCD 2001 is a database developed from a consortium initiated by the U.S. Geological Survey to interpret land-cover attribute categories from Landsat 5 and 7 images (Homer et al. 2004). Pixels from these images were assigned digitized landcover attribute categories with a spatial resolution of $30-\mathrm{m}$. This digitized layer was used to determine the areal proportion of land represented by each category at the two buffers surrounding each site as mentioned previously ( $1-\mathrm{km}$ and $500-\mathrm{m}$ buffers). The proportions were entered as environmental variables into an ordination analysis for species composition at each site to determine which categories correlated best with species composition.

Stream order was determined using ArcGIS 9.0, with first order streams having no permanent tributaries, second order streams occurring at the junction of two first order streams, and third order streams at the junction of two second order streams. Cumulative catchment area was determined by adding subcatchment areas upstream of the site to the area of the subcatchment in which the site was located. This was done because catchment size is believed to affect stream conditions; therefore, it could be important for riparian plant composition. To explore relationships between species identity, percent exotic species, and the measures of community diversity with a measure of socio-economic status of land owners, property values were used, as listed by the Property Valuation Adminstrator offices in Jefferson and Oldham counties. The value of the parcel of land in which the site was located was taken as the property value. In instances where the site was located on the edge of a park abutting residential housing units or businesses, the values of surrounding parcels was used. Where sites were located in the interior of a park or natural area, the property was assigned a value of $\$ 1$. 
Statistical Analyses

Potential relationships between plant species composition, degree of impervious surface, NLCD land-cover categories, and other environmental parameters listed below were explored using non-parametric ordinations, indicator species analysis (Dufrene and Legendre 1997), and cluster analyses. Software packages used were PC-Ord v. 4.41 (McCune and Mefford 1999) and R v.2.11 (R Development Core Team 2009). Nonmetric multidimensional scaling (NMS) in PC-Ord was executed according to the recommended procedure outlined in McCune \& Grace (2002). For each analysis, a separate starting configuration was supplied based on an initial analysis with the following configuration: Sorensen distances, 50 runs with real and random data, a random starting configuration, and 200 iterations to evaluate stability of the final solution (stability criterion $\leq 0.0005$ ). Final solutions were completed using one run with real data. Ordinations run using $\mathrm{R}$ were given an optimal geometric starting configuration based on principal coordinates analysis of the same data. The statistical significance of the indicator value is assessed using a Monte Carlo test for significance, with a p-value indicating the probability of finding the computed stress value for a specific number of dimensions by chance (based on 50 permutations).

Indicator species analysis is a method of determining the value of a species to a particular grouping of sites (Dufrene and Legendre 1997). This method utilizes relative abundance and relative frequency of occurrence of a species within a particular group of sample units to assign a proportional indicator value. The statistical significance of the indicator value is assessed using a Monte Carlo test for significance, with a p-value 
indicating the probability of finding the observed indicator value by chance (based on 1000 permutations).

The goal of cluster analysis was to obtain groupings of research sites based on commonalities in species composition. To do this, a site dissimilarity matrix was constructed based on groundcover species composition. Several dissimilarity measures and clustering techniques were applied to the data in an attempt to achieve the best cluster configuration for the sites. I decided upon complete clustering as it produced the highest within-to-among cluster similarity (Partana ratio) with the most even distribution of sites among clusters. The Bray-Curtis distance measure was used to create a dissimilarity matrix of the sites prior to cluster analysis. To decide on the number of groupings and assess their overall quality, I used the Partana ratio, as mentioned, as well as silhouette plots. Figure 3-3 shows an example of a silhouette plot for three groupings. Silhouette plots assign numerical values (between -1 and 1) termed "silhouette widths" $\left(\mathrm{S}_{\mathrm{i}}\right)$ to each research site (i) within a cluster. The silhouette width is obtained by comparing a research site's mean similarity to other sites within the cluster, followed by comparison to its mean similarity to sites within the nearest cluster. A silhouette width of +1 means the within- cluster similarity is much higher than the between-cluster similarity, indicating a good fit of that site to the cluster. A value of -1 means the between-cluster similarity is much higher than within-cluster similarity, indicating a poor fit of that site to the cluster. An average silhouette width is computed for each cluster in order to compare quality of the groupings. An average silhouette width is determined based on site silhouette widths in order to compare different numbers of groupings, where 
the goal is to obtain a value as high as possible without compromising the quality of the individual clusters.

To determine whether measures of riparian groundcover species abundance and diversity (\% cover, diversity indices) varied with the proportion of subcatchment impervious surface, I used scatterplots and a single factor model (ANOVA, R v.2.11). Scatterplots were generated using all sites, and a subset of sites occurring along first order streams to control for increasing catchment area. ANOVA was used for partitioning variance among land-use categories (urban, suburban, rural), and a post-hoc test (Tukey's HSD pairwise comparisons) was used for determining statistical significance of the variance between pairs of land-use categories. Data were assessed for homoscedasticity prior to analyses and were transformed to better meet those assumptions, if necessary. In addition, any potential outliers were identified using quantile by quantile (Q-Q) plots and graphs of leverage vs. standardized residuals plotted with Cook's distance. Points appearing to assert a disproportional influence on the data were sequentially eliminated from the analysis to assess effects on the statistical assumptions of homoscedasticity and homogeneity of variance. Data points were permanently removed from the analyses if their elimination improved these statistical assumptions.

\section{Results}

Subcatchment delineation \& classification

Forty subcatchments were delineated based on topography within and near the three main catchments. Sites were classified into land use categories based on $\%$ impervious surface cover (ISC) at three scales: subcatchment, $1-\mathrm{km}$ and $500-\mathrm{m}$ radius site 
buffers (Table 3-1). There were twelve urban subcatchments, with one in Goose Creek watershed and eleven in Beargrass Creek watershed. Rural subcatchments included eleven in Harrods and three in Goose Creek. Suburban subcatchments spanned all three catchments, with eight in Goose and three each in Beargrass and Harrods Creek watersheds (Table 3-1). Impervious surfaces within 500-m and 1-km of each site generally decreased compared with values at the subcatchment scale. Therefore, as the scale of the buffer distance around each site decreased, the number of sites classified as urban fell, while sites classified as suburban and rural increased (Table 3-1). One site in Beargrass Creek watershed on a privately owned farm was reclassified as rural when analyzed using the $500-\mathrm{m}$ radius site buffer. The site was kept primarily in mown grass, with a thin strip of woody vegetation allowed to persist adjacent to the creek. All sites with $\geq 30 \%$ ISC within $1-\mathrm{km}$ and $500-\mathrm{m}$ radii of a site were located in Beargrass Creek watershed.

\section{Riparian zone widths}

Riparian width varied with catchment scale land-use. Suburban sites exhibited the smallest mean width, while rural sites had the highest mean/median width and so had the greatest number of multi-plot sites (Table 3-2). Six of fourteen rural sites, four of fifteen suburban and three of twelve urban sites were less than twelve meters wide, resulting in smaller nearstream plot sizes in these sites. Only two urban sites contained more than one plot. One of the sites, located in a city park (Cherokee Park), contained two plots. The other site accomodated three plots, since it extended approximately $100-\mathrm{m}$ before 
intersecting a highway. All suburban sites contained only one plot as all vegetated riparian sites were less than 30 meters wide.

The distribution of riparian widths at urban and rural multi-plot sites was similar (Table 3-2). In rural subcatchments, two of the multi-plot sites were along $1^{\text {st }}$ order reaches, one occurred along a $2^{\text {nd }}$ order reach, and two lay adjacent to $3^{\text {rd }}$ order streams. One of the rural multi-plot sites along a $1^{\text {st }}$ order reach appeared to have an altered stream flowpath, as it was located along a residential street and directly abutted an adjacent upland slope. The two multi-plot sites in the urban subcatchments occurred along $2^{\text {nd }}$ and $3^{\text {rd }}$ order reaches.

The distribution of narrow riparian zones with regard to stream order exhibited greater variation between land-use categories (Table 3-2). Seven of the sites with riparian widths less than 12 meters were found along first and second order streams. In suburban subcatchments, three of the smaller plots were located along $1^{\text {st }}$ order streams, and one was located along a $3^{\text {rd }}$ order stream. Two of the urban riparian sites with riparian widths less than 12 meters were located along $1^{\text {st }}$ order streams, and one along a $3^{\text {rd }}$ order stream. Both of the narrow sites along $3^{\text {rd }}$ order streams (one along urban and one along a suburban stream) appeared to have been dramatically altered, possibly to contain floodwaters. In the urban area, the first terrace out of the stream channel contained a narrow riparian zone $(<12-\mathrm{m})$ which led to a vertical incline and second terrace approximately six feet higher (at the urban site) than the riparian zone. The opposite bank on the other side of the stream channel was practically identical. Such uniformity suggests human intervention. Alterations of this sort, while not surprising, 
carries implications for some of the ecosystem services provided by riparian zones in urban areas.

Variation in Plant Community Structure by Land Use

Across all 41 plots in all three watersheds I found there were 96 groundcover species, with 88 being herbs and 8 woody vines, and with 78 being native and 18 exotic. Across all sites the top four most frequently occurring species were Ageratina altissima, Sanicula odorata, Glechoma hederacea (exotic herb) and Euonymus fortunei (exotic woody vine). A. altissima was the most frequently occurring species in rural and suburban sites, and E. fortunei was the most frequently occurring species in urban sites (site land-use categories determined at the subcatchment scale). E. fortunei also had the highest mean $\%$ cover in urban riparian areas $(16.8 \%)$, which was approximately five times greater than the second most abundant species, A. altissima (3.1\%, Table 3-3). G. hederacea had the highest mean \% cover of all species in suburban areas (8.1\%), which was nearly twice that of the next highest species, E. fortunei (4.3\%). A. altissima was the most abundant species in rural riparian areas (3.9\%), covering approximately the same average area in urban and suburban locations.

In addition to differences in individual species abundances, differences in groundcover species diversity were discovered as well. I found that groundcover species richness was significantly reduced with increasing $\%$ IS at the subcatchment scale $(p=$ 0.003 , adj. $\mathrm{r}^{2}=0.17$; Fig. $3-4$ ). Total species richness across all plots increased from urban to suburban and rural sites from 38 to 58 to 77 species, respectively, with woody vine species remaining fairly constant ( 7 to 8 species) for all land uses (Table 3-3). Other 
diversity indices (Shannon's, Simpson's reciprocal, and Shannon's equitability) did not exibit a linear pattern of change with \% IS, so I explored differences among these measures using categorical analyses. Groundcover plant diversity in urban nearstream plots was lower than that in rural riparian areas. Both Simpson and Shannon's indices showed similar trends at this scale, but only Shannon's index was statistically significant $(p=0.023 ;$ Fig. $3-5 a)$. Shannon's index for rural plots was higher $($ mean $=1.61)$ than for urban plots $(0.99 ; \mathrm{p}=0.02)$. While Simpson's reciprocal index did not differ statistically among land-use categories ( $\mathrm{p}=0.088$; Fig. $3-5 \mathrm{~b})$, it did show a higher mean value in rural plots (mean $=3.88)$ than in urban plots $($ mean $=2.35 ; \mathrm{p}=0.077$ for this two-way comparison). Equitability of the riparian plots did not differ among land-use categories (urban mean $E_{h}=0.53$, suburban and rural means $E_{h}=0.61$; Fig. 3-5c).

Abundance measures of total groundcover and herbs did not show any significant linear or uniform non-linear trends with increasing subcatchment \%IS (Appendix 6). Vine cover increased with increasing impervious surface, but the data were highly heteroscedastic. Percent herbaceous cover declined in a portion of the sites located in subcatchments with greater than $20 \%$ impervious surface cover. Percent vine cover attained a maximum of $70 \%$ where subcatchment impervious surface cover reached $30 \%$, and herb cover steadily declined to below $30 \%$ in those sites with $40 \%$ or more impervious surface cover. Proportions of exotic groundcover (herbs and woody vines) revealed no significant patterns regardless of this uneven distribution of vine and herb cover (Appendix 6).

A subset of sites along first order streams were assessed separately to control for variation in plant community variables related to differences in cumulative catchment 
area. A significant reduction in diversity measures (richness, Shannon's and Simpson's indices) occurred with increasing \%IS cover (determined at 1-km site radius scale; Fig 36). Pearson's r values did not exceed 0.5 for any of the diversity measures. Correlations with $\%$ IS were as follows: Shannon's diversity, $r=0.48(\mathrm{p}=0.01)$; Simpson's diversity, $r=0.41(p=0.02)$; and species richness, $r=0.4(p=0.03)$. Evenness (Shannon's equitability index) did not vary with \% IS, but did show a positive correlation with bank height adjacent to plots along first order streams $(p=0.004$, Pearsons's $r=0.57$; Appendix 7). The pattern, however, seemed driven by a small number of plots at the very low end of bank height $(<50-\mathrm{cm})$ and at the high end $(200-\mathrm{cm})$. The plot with the highest bank height only contained two groundcover species, each having less than $1 \%$ cover, but with approximately equivalent cover values, resulting in a relatively high evenness value. Abundance measures (\% cover) did not show any significant relationships with $\%$ IS; however, woody vine \% cover was greatest within two plots at 25 and $30 \%$ IS cover (determined at the subcatchment scale; Appendix 7). The vine that covered most of the area within those plots was $E$. fortunei.

While species diversity differed among land-use categories, diversity also varied with lateral distance from the stream along the riparian zone. Species richness showed differences between urban, suburban, and rural riparian zones with lateral distance from the stream, although no differences were evident within those land-use categories. In urban subcatchments, species richness was half that of rural subcatchments within 8 meters of the stream (Fig. 3-7, Table 3-4). Urban richness (mean $=4.9$ species) was also significantly lower $(\mathrm{p}=0.037)$ than suburban richness $($ mean $=8.4)$ between 1 and 2 meters from their respective streams. Mean species richness in suburban areas decreased 
by two and three species from the 1-2 meter to 10-11 meter distance from the stream, but remained approximately the same with distance from streams in urban and rural riparian zones. These differences could not be attributed to changes in canopy openness among the intervals, as no significant differences were evident within or among land-use categories in canopy openess with increasing distance from the stream. Two urban and two suburban nearstream plots were partially covered by lawn. One plot each in both urban and suburban categories contained lawn between 4 and 11 meters from the stream. A second site in both land-use categories contained lawn between 7 and 11 meters from the stream. A third urban plot was reduced to a single species (Eunonymus fortunei) at the 7-8 meter interval. Groundcover richness with distance from the stream appears more stable in urban and rural riparian zones than in suburban riparian zones, but with a lower mean richness in urban and a higher mean richness in rural riparian zones.

Riparian plant species richness also differed with downstream distance within land-use categories. I found a significant positive relationship between species richness in nearstream plots and cumulative catchment area within suburban riparian zones (Fig. 38). In contrast, no significant linear relationship was detected between these two variables within urban or rural land-use categories. Bank height adjacent to the plot was also used as an integrative index of flood disturbance, but no significant linear relationships were apparent with either groundcover species richness or other community diversity indices.

\section{NMS \& Cluster analysis - Groundcover species}

I conducted a cluster analysis to obtain groupings of research sites based on commonalities in species composition. Cluster analysis of the groundcover layer resulted 
in three clusters of 39 sites (out of 41) using complete clustering and Bray-Curtis distance. Two sites were not used since each one formed a separate cluster, indicating they were not a good fit with any of the other sites. The Partana ratio was 1.74 with an average silhouette width of 0.11 . Cluster- 1 contained the greatest number of sites (nineteen) with an average silhouette width of 0.13 . Cluster- 2 contained the fewest sites (eight) with an average width of 0.12 . Cluster-3, with twelve sites, was the weakest cluster with an average silhouette width of 0.06 .

Clusters were analyzed for differences in species composition using frequency of occurrence within sites making up the clusters and indicator species analysis. Those species with significant indicator values in each of the clusters $(p \leq 0.05)$ are shown in Table 3-5. Assemblage identity of cluster-1 was defined by the indicator species Glechoma hederacea (an exotic herb) and non-lawn (unmanaged) grasses. However, the grasses, Microstegium vimineum and Elymus riparius, were not included in this category as I was able to identify them to species. Other noteworthy species occurring with greater frequency though not exclusively in this cluster were the two native species, Verbesina alternifolia and Impatiens capensis, a wetland species. All sites in cluster- 2 contained the indicator species Euonymus fortunei, an exotic vine that also occurred in cluster-1 with lesser frequency. The native woody vine, Toxicodendron radicans, was ubiquitous, but occurred with the greatest frequency in cluster-2. A mix of species defined cluster-3, as all indicator species occurred with a frequency of less than 0.5. Lysimachia nummularia, Symphiotrichum cordifolium, Juncus spp., and Packera obovata were present in at least $25 \%$ of all sites in this cluster. L. nummularia and Juncus spp. are classified as wetland (facultative or obligate) species, and all these species are native. 
Non-metric multidimensional scaling (NMS) was used to determine cluster associations with environmental variables and species attributes. NMS was run using the 39 sites organized into a distance matrix (using Sorensen's index) based on groundcover species importance values (IVs). A 3-dimensional configuration was chosen, achieving a final stress of 16.6 and instability of 0.00048 for the final configuration. When sites were assigned to their respective clusters, no site overlap was visible among the three clusters in 3-dimensional space, indicating satisfactory agreement between the cluster analysis and the NMS solution (Fig. 3-9). Clusters were analyzed in relation to environmental variables that might correlate with and potentially help explain the observed variation in species composition among clusters (Table 3-6). Environmental variables calculated for each site were entered into a site-by-variable matrix and included as a secondary matrix in NMS using PC-Ord. The biplot feature in PC-Ord was used to generate a line for each variable with an $\mathrm{r}^{2} \geq 0.15$ for one or more axes, with the direction of the line corresponding to the direction of positive correlation of the variable and the length of the line indicating the strength of the correlation along one or more axes. Implied in the ordination plot but not visible, a vector of equal length but opposing sign points in the opposite direction to indicate a negative correlation.

NLCD categories and \% impervious surface at various scales were included in the NMS as environmental variables to evaluate cluster identities with fine and coarse scale land-cover variables (Table 3-6; Fig. 3-10). The importance value of $L$. maackii for each site was included as an environmental variable to determine if the species might be a possible influence on groundcover species composition. Cluster-2 in the 3-cluster configuration was the most "urban" of the clusters, being most strongly and positively 
correlated with \% IS (ranging between $12 \%$ and $48 \%$ ) within a $1-\mathrm{km}$ radius of each site center, as indicated by the length of its vector (Fig. 3-10a,b). Since impervious surface cover at the 1-km scale showed the strongest correlation with the NMS axes, NLCD database land-cover categories at that scale were used for further analyses. Low-intensity developed land most strongly correlated with this cluster (Fig. 3-10c,d). This type of land-cover is typically composed of single-family residential housing, which was the most frequently observed land-cover type found in this study. To a lesser extent, medium-intensity and open-space developed land also positively correlated with the species composition of cluster-2, followed by high-intensity developed land, which occurred around a few of the most urban sites. Lonicera maackii was slightly more important in explaining cluster- 2 than the other clusters. While all clusters contained sites with honeysuckle, most sites within cluster-2 had an L. maackii IV greater than 75 . Only one site contained no honeysuckle (IV $=0$ ), and two sites had a Lonicera IV of 200 (maximum IV). Clusters-1 and -3 were most positively correlated with the proportion of deciduous forest cover and pasture (Fig. 3-10c-d). Cluster-1 contained between $8 \%$ and $82 \%$ deciduous forest cover and cluster-3 between $18 \%$ and $68 \%$ deciduous forest cover within a $1-\mathrm{km}$ radius of the sites. Pasture comprised between $0 \%$ and $39 \%$ cover in both clusters. Wetland cover was located primarily within a radius of cluster-1 sites, with eight of the sites having between 0.1 and $0.5 \%$ cover for this habitat type. Most sites in clusters- 1 and -3 contained less than $24 \%$ IS cover within a 500-m radius, but three sites contained up to $45 \%$. Low-intensity developed land cover occurred with a proportion up to $27 \%$ around the sites in these two clusters. While primarily located within a mixture of 
deciduous forest and pasture, the influence of urban development was therefore detectable.

Species attributes (Appendix 2) were included in the analyses as environmental variables to assess which attributes associated with the same site clusters as the landcover variables. In this way an indirect correlation could be drawn between species attributes and land-cover variables. Figure 3-11 shows the direction of correlation of these attributes in relation to the various site clusters. Obligate wetland (OBL) and native species attributes most strongly correlated with clusters- 1 and -3 . Facultative species (FAC) did not positively associate with any particular grouping of sites. The vector for exotic species (E) extended in opposition to the native vector $(N)$, correlating most strongly with Clusters-1 and -2 .

L. maackii and E. fortunei analysis

Since L. maackii and E. fortunei were important species associated with urban clusters, I decided to determine if their distributions and densities were related. Only nearstream plots were used for these analyses. First, all plots containing E. fortunei were included in the analysis, regardless of whether they contained L. maackii (Fig. 3-12a). A strong positive correlation was shown between $L$. maackii stem density and E. fortunei percent cover (Pearson's $r=0.73, p=0.0001$ ). When only plots containing both species were included in the regression (Fig 3-12b), an even stronger positive correlation emerged (Pearson's $r=0.81, \mathrm{p}=0.001$ ).

I then determined whether measures of groundcover species diversity in nearstream plots decreased with increasing density and cover of $L$. maackii and $E$. 
fortunei. Where E. fortunei was used as a correlate with diversity measures, that species was not used in calculations of species diversity. In plots containing L. maackii, a weak negative correlation was found between L. maackii density and groundcover species richness (adj. $r^{2}=0.19, p=0.019 ;$ Fig. 3-13a) and Shannon diversity (adj. $r^{2}=0.20, p=$ 0.012; Fig. 3-13b). No trends were detected between L. maackii density and Shannon's equitability index. However, \% cover of $E$. fortunei was negatively, but weakly, correlated with Shannon's equitability index (adj. $r^{2}=0.12, p=0.06$; Fig. 3-14). Relationships with Shannon's index, Simpson's reciprocal index, and species richness can be seen in Figure 3-15. Values of $E$. fortunei $\%$ cover above $30 \%$ showed consistently low levels of diversity.

\section{Discussion}

Exotic woody and herbaceous vine species were the most abundant groundcover occurring in urban and suburban riparian zones. E. fortunei occurred with an average site cover of $17 \%$ in urban riparian sites, and $G$. hederacea covered $8 \%$ of suburban riparian sites. E. fortunei was the second most abundant species in suburban riparian zones, with an average cover of $4.3 \%$. The dominant cover in rural riparian zones, the native herb $A$. altissima, also occurred in urban and suburban riparian sites with approximately the same average cover (rural mean $=3.9 \%$, suburban mean $=3.3 \%$, urban mean $=3.1 \%$ ). Urban and suburban areas are notorious for their associations with exotic species. These findings indicate that while these areas are more susceptible to invasion by exotic species, native species that are abundant in rural environments are not necessarily being excluded from urban and suburban riparian zones. 
Species richness of all groundcover species significantly declined with increasing subcatchment impervious surface (Fig. 3-4 and 3-6a). This differed from my original prediction of greater richness in surburban areas in that richness was just as high or higher at the rural end of the gradient. This may indicate more resource heterogeneity or less stressful conditions across rural riparian areas enabling support of a greater number of species. Reduction in groundcover species richness along the gradient could also be partially attributed to the densities of $L$. maackii, which I (Fig. 3-13a) and others (Luken and Thieret 1996, Hutchinson and Vankat 1997, Borgmann and Rodewald 2005, Castellano and Boyce 2007) have shown to be associated with reduced richness of groundcover plants. I had shown in a study of woody vegetation at these same sites that L. maackii importance value was significantly positively correlated with proportion of impervious surface within a 500-m site radius (See Fig. 2-17 in Dissertation Chapter 2), demonstrating it to be a dominant riparian species in urban Louisville.

Other measures of species diversity were used as response variables to gauge how degree of subcatchment urbanization affects groundcover plant communities. For nearstream plots both Shannon's and the Simpson's reciprocal indices of diversity were lowest for urban and highest for rural subcatchments (Fig. 3-5). Scatterplots from riparian sites along first order streams also supported this pattern (Fig. 3-6b-c). Since Shannon's equitability index did not differ between land-use categories, differences in the indices were primarily due to differences in species richness. While fewer groundcover species exist in urban riparian areas than in suburban or rural riparian areas, the species that did exist are distributed in similar proportions, regardless of surrounding land-cover/land use. Comparison of the groundcover layer among land-use categories supports the conclusion 
that vegetation in rural riparian zones is generally more diverse than in urban riparian zones.

As resources and environmental conditions can change within a riparian zone laterally as well as longitudinally up and downstream, I predicted that groundcover species diversity would vary with distance from the streambank. No significant differences were found between distance intervals within land-use categories. However, when comparing distance intervals between land-use categories, urban richness was shown to be significantly lower than rural richness within eight meters of the stream, but not beyond that distance. Urban richness was also lower than in suburban plots within two meters of the stream bank edge (Table 3-4, Fig. 3-7). These findings suggest that, in addition to the availability of a larger species pool, nearstream conditions within rural riparian zones could be a factor favoring higher species diversity than those in urban riparian zones. Suburban riparian zones possess a greater species pool only at the distance interval nearest the stream. As distance from the streambank increases, suburban riparian plant diversity becomes more similar to urban diversity. However, this low suburban diversity pattern was in part due to the presence of lawns, which I considered as monocultures within urban and suburban riparian sites. Lite et al. (2005) also found decreasing herbaceous species richness with increasing distance from the stream channel in an arid environment in Arizona. Following rains and flooding at the same sites, the pattern was reversed, with herbaceous richness increasing farther from the stream. These patterns were attributed to changes in soil water availability and drainage. Therefore, variation in soil water availability is one possible explanation for richness differences between urban, suburban, and rural areas in this study. I measured streambank height as a 
proxy for groundwater levels in all plots, and actually measured depth to groundwater in a subset of nearstream plots to examine whether relationships existed between these variables and variation in riparian plant communities. The highest measured streambank heights occurred adjacent to urban plots $($ mean $=122-\mathrm{cm}$, vs. 96- and 86-cm in rural and suburban plots, respectively). Streambank height and mean depth to groundwater (see Dissertation Chapter 4) could then partially explain the lower richness at urban sites at least within the 1-2 meter interval. Mean depth to groundwater was found to be lower along urban riparian zones than suburban or rural riparian zones over a period of one year (see Dissertation Chapter 4). The contrast was most stark in the spring, when rainfall allowed recharge of rural and suburban groundwater tables but not urban water tables (see Fig. 4-4 in Dissertation Chapter 4). Further research would need to be conducted in these areas to determine whether groundwater decreased or increased with lateral distance from the stream to more fully understand how this variable might affect herb and woody vine cover in riparian areas along urban-to-rural gradients.

Upstream-to-downstream (longitudinal) gradients in plant species diversity have also been shown to exist in riparian environments. Since flooding intensities and frequencies vary with upstream catchment area, it is a likely contributor to the differences in richness seen with longitudinal distance downstream. As such, I used cumulative upstream catchment area in lieu of longitudinal downstream distance, even though this measure has been referenced in previous studies (Bendix 1997, Bendix and Hupp 2000, Lite et al. 2005). My prediction of higher species richness with greater upstream catchment area was only partially supported. While cumulative upstream catchment area was significantly and positively correlated with groundcover species richness within the 
suburban land-use category (Fig. 3-8), it was not a predictor of species richness for riparian areas within either urban or rural land uses. Several streamflow parameters, which may have contributed to this pattern, have been shown to increase or decrease with increasing catchment impervious surface cover (Paul and Meyer 2001). These parameters include increases in bankfull and peak discharge, and decreases in lag time. As catchment area increases moving downstream, disturbance to the riparian zone can increase as flood velocity and volume increases (Bendix 1997). Although bank height increases with increasing upstream catchment area, no trends were detected between bank height and groundcover richness. Stream channel structure and width-to-depth ratios are additional measures that should be compared and which might explain this species richness phenomenon, as both could affect severity of flooding.

\section{Plant community analysis}

Site groupings via cluster analyses and NMS generally supported my prediction of distinct vegetation communities being correlated with impervious surface cover. Three communities were defined and analyzed for correlations with environmental factors and species attributes. Cluster-1 contained the greatest proportion of sites with surrounding wetland cover and was defined by unmanaged grasses and the exotic species $G$. hederacea (Table 3-5). The most urban cluster (cluster-2) was defined by the exotic species E. fortunei (Fig. 3-10). Although I expected vegetation communities to be most strongly correlated with impervious surface at the smallest scale measured (500-m site radius), this was not the case. This cluster correlated most strongly with measures of impervious surface and urban land cover within a 1-km radius of the sites. 
Environmental correlates with the three clusters suggest they are composed of groundcover species with differential affinities for site conditions associated with proportions of impervious surface and deciduous forest cover, with wetland species possessing low affinities for surrounding impervious surfaces and certain exotic species exhibiting higher affinities. These findings provide partial support for my predictions regarding exotic vs. native and wetland vs. non wetland species. While some species associations correlated to a greater degree with impervious surface and urban land cover, no clusters showed complete fidelity to urban vs. suburban vs. rural categories, as each cluster contained several sites with at least moderate proportions of impervious surface and urban cover within a $1-\mathrm{km}$ radius. Furthermore, the absence of strong patterns regarding exotic species richness and abundance proportions along an impervious surface gradient (Appendix 6) suggests the "urbanness" of an area does not necessarily predict the presence or abundance of exotic species. However, cluster correlations with the exotic shrub, L. maackii, suggest that this species influences groundcover species at the plot scale, no matter what land-cover type the plot was surrounded by within a 1-km radius.

\section{Groundcover plant associations with shrub honeysuckle}

In another study (see Dissertation Chapter 2), I found a strong positive correlation between importance value of the exotic shrub, L. maackii, in plots and impervious surface cover within a $500-\mathrm{m}$ radius of research sites. In this study I also found that the exotic woody groundcover vine, E. fortunei, often co-occured with this shrub. The percent cover of E. fortunei dramatically increased in the presence of high densities of L. maackii (Fig. 3-12), approaching 70\% cover at the highest densities of the shrub. Where L. maackii was 
not present, E. fortunei percent cover did not exceed 5\% cover (Fig. 3-12a). Castellano and Boyce (2007) discovered a very different pattern regarding L. maackii and Juniperus virginiana along a road cut in northern KY, USA. Both species were found to coexist at the same site, where L. maackii demonstrated a clumped distribution, and J. virginiana exhibited a more random distribution. These two species were able to co-exist since they were able to partition resources between themselves at the same sites, with $J$. virginiana inhabiting areas with higher photosynthetically active radiation, indicating lower shade tolerance. In the case of riparian sites in this study, E. fortunei appears to thrive beneath dense L. maackii clusters, suggesting either that L. maackii may be facilitating growth of this species where they occur together, or conversely E. fortunei may promote successful germination and growth of L. maackii.

I also examined whether measures of groundcover species diversity decreased with increasing density and cover of L. maackii and E. fortunei. Both groundcover species richness and Shannon's index decreased with increasing densities of L. maackii (Fig. 3-13). This decreasing pattern of plant species richness with increasing exotic cover was also shown by Loewenstein and Loewenstein (2005), where understory plant species richness (saplings, shrubs, herbs, vines) decreased with increasing cover of the exotic shrub Ligustrum sinense in riparian forests near Columbus, Georgia, USA. In the Louisville sites, Shannon's equitability index was negatively but weakly correlated with E. fortunei percent cover (Fig. 3-14), but no correlation was found with species richness. However, in sites where this vine exceeded $30 \%$ cover groundcover diversity measures were all relatively low. While L. maackii and E. fortunei may co-occur, they appeared to interact differently with groundcover plants, with elimination of species under L. maackii, 
and changes in species proportions where $E$. fortunei occurs. These results help explain variation in composition of species assemblages within the clusters. Cluster-2 was defined by $E$. fortunei, and nearstream equitable distributions of species declined as $E$. fortunei cover increased. Since E. fortunei cover increases in relation to L. maackii density, the shrub layer also helps to define groundcover communities in the more urban riparian sites where L. maackii dominated the shrub community (see Dissertation Chapter 2), lending support to my predictions that $L$. maackii is involved in structuring the plant community.

\section{Conclusions}

I found that urbanization in the Louisville Metro area is associated with decreasing diversity of riparian groundcover plant communities, reduced importance of obligate wetland species, and increased importance of the exotic vine, E. fortunei, a species sold by nurseries in the area for residential and commercial plantings. In addition, this study demonstrates the negative effects that the exotic shrub, L. maackii, is likely having on riparian herb and woody vine diversity. The co-occurrence of both these exotic species may be synergistically decreasing native species diversity in urban riparian sites. Furthermore, declining forested buffers reduce both riparian and instream diversity. I found presence of managed vegetation (lawns) contributed to the declining diversity of suburban riparian zones moving laterally from the stream. Moore and Palmer (2005) found declining instream invertebrate diversity with a declining proportion of forested riparian areas. These findings carry important implications since they show high amounts of impervious surface are not necessarily needed to affect riparian and instream diversity. 
Simply reducing the complexity of riparian vegetation is sufficient to negatively affect this aspect of riparian environments. Also found in suburban riparian sites, increasing cumulative catchment area results in increasing species richness in but did not appear to explain species richness variation in urban and rural sites. Further research should concentrate on assessing the intensity and frequency of floodwaters in suburban environments, and assessing how they differ from urban and rural locations as upstream catchment area increases.

These findings suggest a change is needed on the conceptual model in Figure 1-1, with addition of an intermediary between the water table and riparian groundcover (Fig. 3-16). Although I found no direct link between bank height (and thus the water table) and species composition or richness, decreasing plant richness has been shown in arid environments with increasing depth to water table (Lite et al. 2005). However, I did find indirect evidence of a soil moisture difference with changes in proportion of impervious surface. Obligate wetland species were rare in most urban communities (Fig. 3-11), assuming greater importance in a more forested environment. Facultative and obligate wetland species are indicators of wetter soils at critical times of the year needed for germination and establishment. Therefore, a soil moisture gradient among sites is implied via the presence of these species in less urban environments. Therefore, I propose the changes in Figure 3-16 to better reflect potential conditions contributing to species composition in this system. Results from the woody species layer also support this model since facultative wetland species were more highly associated with more rural site clusters (see Fig. 2-14, 2-15, and 2-16 in Dissertation Chapter 2). 
Table 3-1: Area of research catchments and the number of study sites classified as urban, suburban and rural at the subcatchment scale, and within a $1-\mathrm{km}$ and $500-\mathrm{m}$ radius from each study site. Classification as urban, suburban and rural land cover was based on proportion of impervious surface with $\geq 30 \%$ being urban $(\mathrm{U}), \leq 10 \%$ being rural $(\mathrm{R})$, and between 10 and $30 \%$ being suburban.

\begin{tabular}{l|c|ccc|ccc|ccc}
\multicolumn{2}{l|}{} & \multicolumn{3}{|c|}{$\mathbf{S C}$} & \multicolumn{3}{c|}{$\mathbf{~ k m}$} & \multicolumn{3}{|c}{$\mathbf{5 0 0} \mathbf{~ m}$} \\
\hline Catchment & $\begin{array}{l}\text { Area } \\
\left(\mathbf{k m}^{2}\right)\end{array}$ & $\mathbf{U}$ & $\mathbf{S}$ & $\mathbf{R}$ & $\mathbf{U}$ & $\mathbf{S}$ & $\mathbf{R}$ & $\mathbf{U}$ & $\mathbf{S}$ & $\mathbf{R}$ \\
\hline $\begin{array}{l}\text { Beargrass } \\
\begin{array}{l}\text { Creek } \\
\text { Goose }\end{array}\end{array}$ & 65 & 11 & 3 & 0 & 8 & 6 & 0 & 6 & 7 & 1 \\
$\begin{array}{l}\text { Creek } \\
\text { Harrods } \\
\text { Creek }\end{array}$ & 50 & 1 & 8 & 3 & 0 & 8 & 4 & 0 & 6 & 6 \\
60 & 0 & 3 & 11 & 0 & 1 & 13 & 0 & 2 & 12
\end{tabular}


Table 3-2: Distribution of riparian width intervals in this study by stream order and landuse category at the subcatchment scale. Riparian zone width was measured along areas of level ground beginning at the streambank edge and extended until reaching a structural barrier (such as a road, parking lot, or building), upland slope, or other obstruction (grazed pasture, ephemeral stream channel). Classification as urban, suburban and rural land cover was based on proportion of impervious surface with $\geq 30 \%$ being urban, $\leq 10 \%$ being rural, and between 10 and $30 \%$ being suburban.

\begin{tabular}{lccccc} 
& \multicolumn{5}{c}{$\begin{array}{c}\text { Riparian } \\
\text { width (m) }\end{array}$} \\
Land use & $\begin{array}{c}\text { Stream } \\
\text { Order }\end{array}$ & $\mathbf{1 2}$ & $\mathbf{1 2 - 4 5}$ & $\mathbf{4 6 - 8 5}$ & $>\mathbf{8 5}$ \\
\hline Urban & $\mathbf{1}$ & 2 & 4 & 0 & 0 \\
& $\mathbf{2}$ & 0 & 1 & 0 & 1 \\
& $\mathbf{3}$ & 1 & 2 & 1 & 0 \\
\cline { 2 - 6 } Total & & 3 & 7 & 1 & 1 \\
\hline Suburban & $\mathbf{1}$ & 3 & 7 & 0 & 0 \\
& $\mathbf{2}$ & 0 & 4 & 0 & 0 \\
Total & $\mathbf{3}$ & 1 & 0 & 0 & 0 \\
\hline Rural & $\mathbf{1}$ & 4 & 11 & 0 & 0 \\
& $\mathbf{2}$ & 2 & 2 & 2 & 0 \\
& $\mathbf{3}$ & 0 & 1 & 1 & 0 \\
Total & & 6 & 3 & 5 & 0
\end{tabular}


Table 3-3: Mean \% cover ( + S.E.) for the top ten most abundant herb and woody vine species in urban, suburban and rural land-use categories.

\begin{tabular}{|c|c|c|c|}
\hline Species & $\begin{array}{c}\text { Urban } \\
\text { plots } 1 \\
\text { Mean \% } \\
\text { cover } \\
\text { (S.E.) } \\
\end{array}$ & $\begin{array}{c}\text { Suburban } \\
\text { plots } \\
\text { Mean \% } \\
\text { cover } \\
\text { (S.E.) } \\
\end{array}$ & $\begin{array}{c}\text { Rural } \\
\text { plots } 3 \\
\text { Mean \% } \\
\text { cover } \\
\text { (S.E.) } \\
\end{array}$ \\
\hline $\begin{array}{l}\text { Ageratina altissima (L.) King \& H.E. } \\
\text { Robins. }\end{array}$ & $3.1(1.5)$ & $3.3(1.4)$ & $3.9(2.4)$ \\
\hline Alliaria petiolata (Bieb.) Cavara \& Grande & $1.8(1.2)$ & $0.76(0.71)$ & 0 \\
\hline Asarum canadense $\mathrm{L}$. & $0.26(0.18)$ & 0 & 0 \\
\hline Carex blanda Dewey & $1.0(1.0)$ & 0 & 0 \\
\hline Euonymus fortunei (Turcz.) Hand.-Maz.* & $16.8(6.9)$ & $4.3(2.2)$ & $0.94(0.91)$ \\
\hline Glechoma hederacea L. & $1.6(0.9)$ & $8.1(3.4)$ & $3.3(1.7)$ \\
\hline Impatiens capensis Meerb. & $0.28(0.24)$ & 0 & 0 \\
\hline Solidago spp. L. & $0.92(0.41)$ & $1.4(0.71)$ & 0 \\
\hline Urtica dioica $\mathrm{L}$. & $0.59(0.32)$ & 0 & $2.7(2.7)$ \\
\hline Verbesina alternifolia (L.) Britt. ex Kearney & $2.1(1.4)$ & $1.4(0.72)$ & $0.71(0.33)$ \\
\hline Arundinaria gigantea (Walt.) Muhl. & 0 & $1.8(1.3)$ & 0 \\
\hline Microstegium vimineum (Trin.) A. Camus & 0 & $3.4(2.6)$ & $3.2(1.4)$ \\
\hline Parthenocissus quinquefolia (L.) Planch. & 0 & $1.1(0.90)$ & 0 \\
\hline Viola spp. L. & 0 & $1.2(0.77)$ & $0.58(0.21)$ \\
\hline $\begin{array}{l}\text { Sanicula odorata (Raf.) K.M. Pryer \& L.R. } \\
\text { Phillippe }\end{array}$ & 0 & 0 & $1.3(0.57)$ \\
\hline Securigera varia (L.) Lassen* & 0 & 0 & $0.68(0.46)$ \\
\hline Vinca minor L.* & 0 & 0 & $0.93(0.93)$ \\
\hline $\begin{array}{l}\text { Mean \% cover (herb and woody vine } \\
\text { species)! }\end{array}$ & $30.1(6.8)$ & $32.5(6.1)$ & $24.6(5.1)$ \\
\hline Mean $\%$ cover (herbs only)! & $13(3.7)$ & $25.8(5.9)$ & $21.7(5.2)$ \\
\hline Herb species richness & 31 & 50 & 70 \\
\hline Woody vine species richness & 7 & 8 & 7 \\
\hline
\end{tabular}

$1 \mathrm{n}=12,2 \mathrm{n}=15,3 \mathrm{n}=14$ plots

*Woody vines

Land-use categories based upon \% impervious surface at the subcatchment scale.

Urban $\geq 30 \%$, Rural $\leq 10 \%$, Suburban between 10 and $30 \%$ IS.

: No significant differences were detected (ANOVA) among land-use categories regarding groundcover $(p=0.63)$ or herb-only $(p=0.24)$ mean $\%$ cover $(\arcsin$ transformed). 
Table 3-4: Riparian groundcover mean species richness by distance from the stream bank and land-use categories determined at the subcatchment scale.

\begin{tabular}{|c|c|c|c|c|}
\hline & $\begin{array}{l}\text { Distance inter } \\
\text { stream }\end{array}$ & from the & & \\
\hline $\begin{array}{l}\text { Land } \\
\text { cover } \\
\text { category }\end{array}$ & 1-2 meters* & 4-5 meters* & $7-8$ meters & $10-11$ meters \\
\hline Rural & $9.9(\mathbf{0 . 0 0 9})$ & $9.1(\mathbf{0 . 0 2 5})$ & $8.6(\mathbf{0 . 0 2 9})$ & 9.3 \\
\hline $\mathrm{n}=$ & 14 & 14 & 10 & 8 \\
\hline Suburban & $8.4(\mathbf{0 . 0 3 7})$ & 7.2 & 6.9 & 5.9 \\
\hline $\mathrm{n}=$ & 15 & 13 & 12 & 12 \\
\hline Urban & 4.9 & 5.0 & 4.2 & 4.8 \\
\hline $\mathrm{n}=$ & 12 & 12 & 9 & 8 \\
\hline \multicolumn{5}{|c|}{$\begin{array}{l}\text { *Species richness was natural } \log \text { transformed for } 1-2-\mathrm{m} \text { and } 4-5-\mathrm{m} \\
\text { intervals for analysis, but values in the table are untransformed } \\
\text { p-values (in parentheses) represent significant differences (at } \alpha=0.01 \text { or } \\
0.05 \text { ) from urban riparian richness }\end{array}$} \\
\hline
\end{tabular}


Table 3-5: Frequently occurring and significant indicator species (using indicator species analysis (Dufrene and Legendre 1997)) for 3-cluster configuration using complete clustering. Frequency values were calculated based on the proportion of sites within the cluster in which the species occurred. Indicator values (IV) were calculated using indicator species analysis, where a value of 0 means the species is never present in the cluster and a value of 1 means the species is always present and exclusive to the cluster. Probabilities for the indicator values were determined using a Monte Carlo randomization technique.

\begin{tabular}{|c|l|l|c|c|c|}
\hline Cluster & Species & Common name & Freq. & IV & p-value \\
\hline $\mathbf{1}$ & Glechoma hederacea & Creeping Charlie & $\mathbf{1}$ & $\mathbf{0 . 9 6}$ & 0.001 \\
\hline & Unmanaged grass & Grass & $\mathbf{0 . 6 3}$ & $\mathbf{0 . 5 1}$ & 0.01 \\
\hline & Verbesina alternifolia & Wingstem & $\mathbf{0 . 7 3}$ & & \\
\hline & Impatiens capensis & Jewelweed & $\mathbf{0 . 5 1}$ & & \\
\hline $\mathbf{2}$ & Euonymus fortunei & Winter creeper & $\mathbf{1}$ & $\mathbf{0 . 7 3}$ & 0.001 \\
\hline & $\begin{array}{l}\text { Toxicodendron } \\
\text { radicans }\end{array}$ & Poison ivy & $\mathbf{0 . 6 2}$ & & \\
\hline $\mathbf{3}$ & $\begin{array}{l}\text { Lysimachia } \\
\text { nummularia }\end{array}$ & Creeping Jenny & $\mathbf{0 . 5}$ & $\mathbf{0 . 4 2}$ & 0.026 \\
\hline & $\begin{array}{l}\text { Symphiotrichum } \\
\text { cordifolium }\end{array}$ & $\begin{array}{l}\text { Common blue } \\
\text { wood aster }\end{array}$ & $\mathbf{0 . 3 3}$ & $\mathbf{0 . 2 9}$ & 0.022 \\
\hline & Juncus spp. & Rush & $\mathbf{0 . 2 5}$ & $\mathbf{0 . 2 2}$ & 0.041 \\
\hline & Packera obovata & $\begin{array}{l}\text { Roundleaf } \\
\text { ragwort }\end{array}$ & $\mathbf{0 . 2 5}$ & $\mathbf{0 . 2 2}$ & 0.043 \\
\hline
\end{tabular}


Table 3-6: Environmental and National Land Cover Database (NLCD) metrics used for non-metric multidimensional scaling.

\begin{tabular}{l|l} 
Environmental variables & NLCD values (500-m) \\
\hline -Bank height adjacent to the site & -Open-space developed \\
$-\%$ impervious surface: subcatchment, & \\
1-km buffer, 500-m buffer & -High-, medium, low-intensity developed \\
-Plot size and distance from channel & -Deciduous forest \\
-Stream order & -Mixed forest \\
-Catchment Area & -Herbaceous forest \\
-Property value & -Crops \\
-Earthworm species number \& weight & -Pasture \\
-Lonicera maackii IV & -Wetland
\end{tabular}




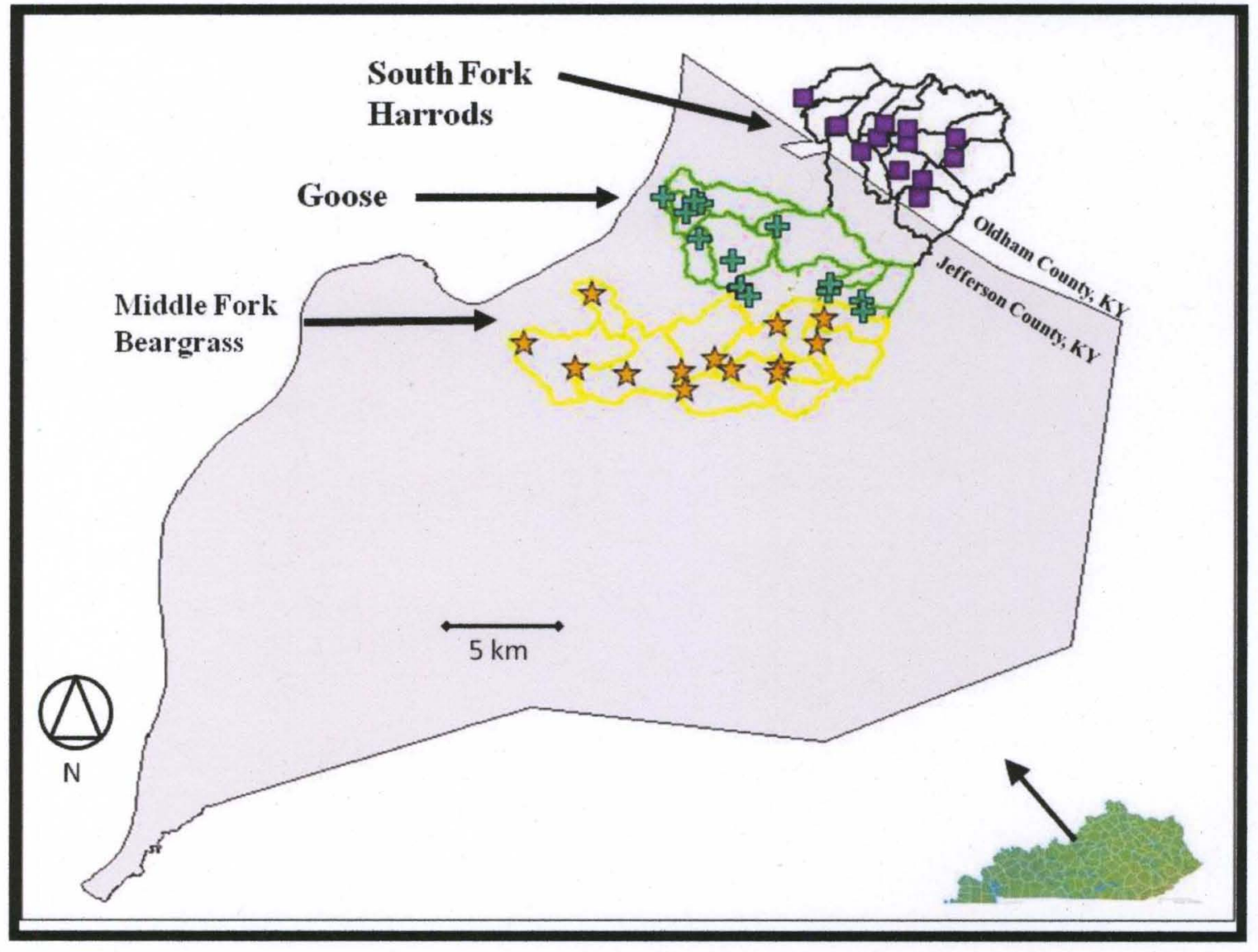

Figure 3-1: Schematic map of the three watersheds in Jefferson and Oldham counties in Kentucky. Research sites are indicated with markers. 


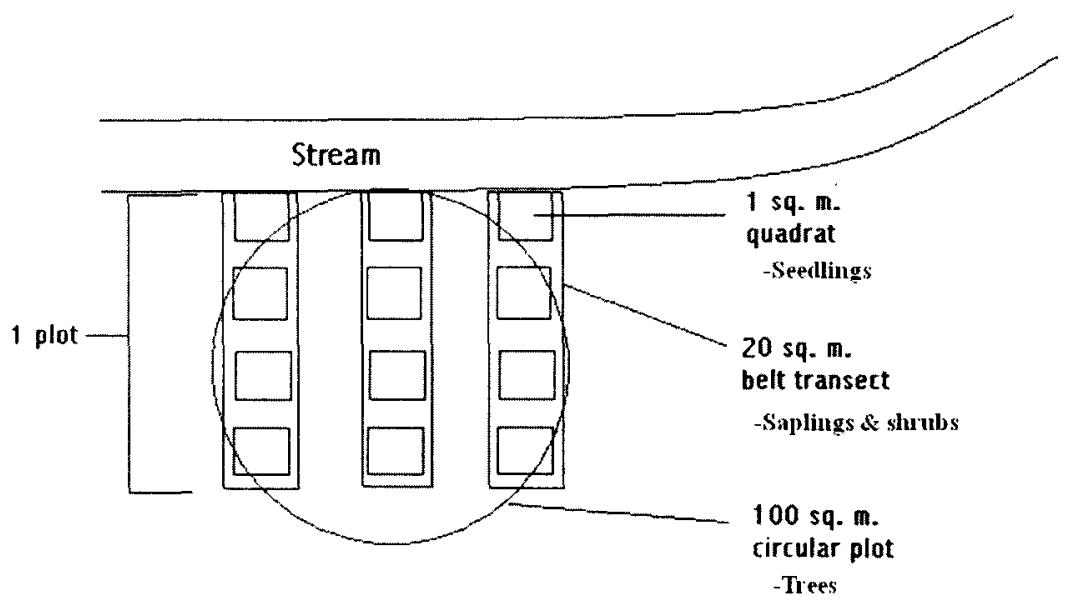

(a)

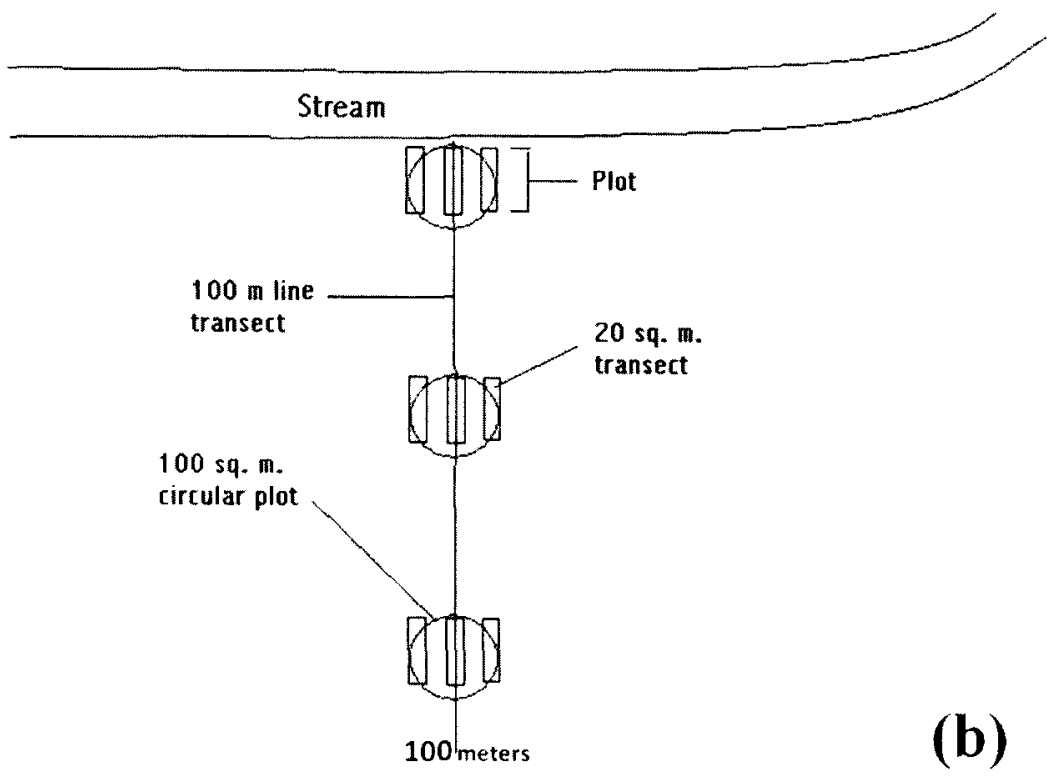

Figure 3-2a-b: Plot and site structure for sampling adult trees $(\geq 2.54 \mathrm{~cm} \mathrm{DBH})$, saplings, tree seedlings, and shrubs. (a) Plots consisted of a $100-\mathrm{m}^{2}$ circular plot for sampling tree counts and DBH. Nested within the circular plot were three belt transects measuring $10 \times 2 \mathrm{~m}$ with midlines spaced four meters apart for sampling sapling and shrub counts. Quadrats measuring $1 \times 1 \mathrm{~m}$ were nested within the belt transects for determining percent cover and counts of tree seedlings. Adjacent quadrat spacing within belt transects was three meters at the midpoint. (b) Sites consisted of a midline transect stretched the length of the riparian zone up to 100 meters with up to 3 plots per transect with midpoints spaced $40-\mathrm{m}$ apart. 


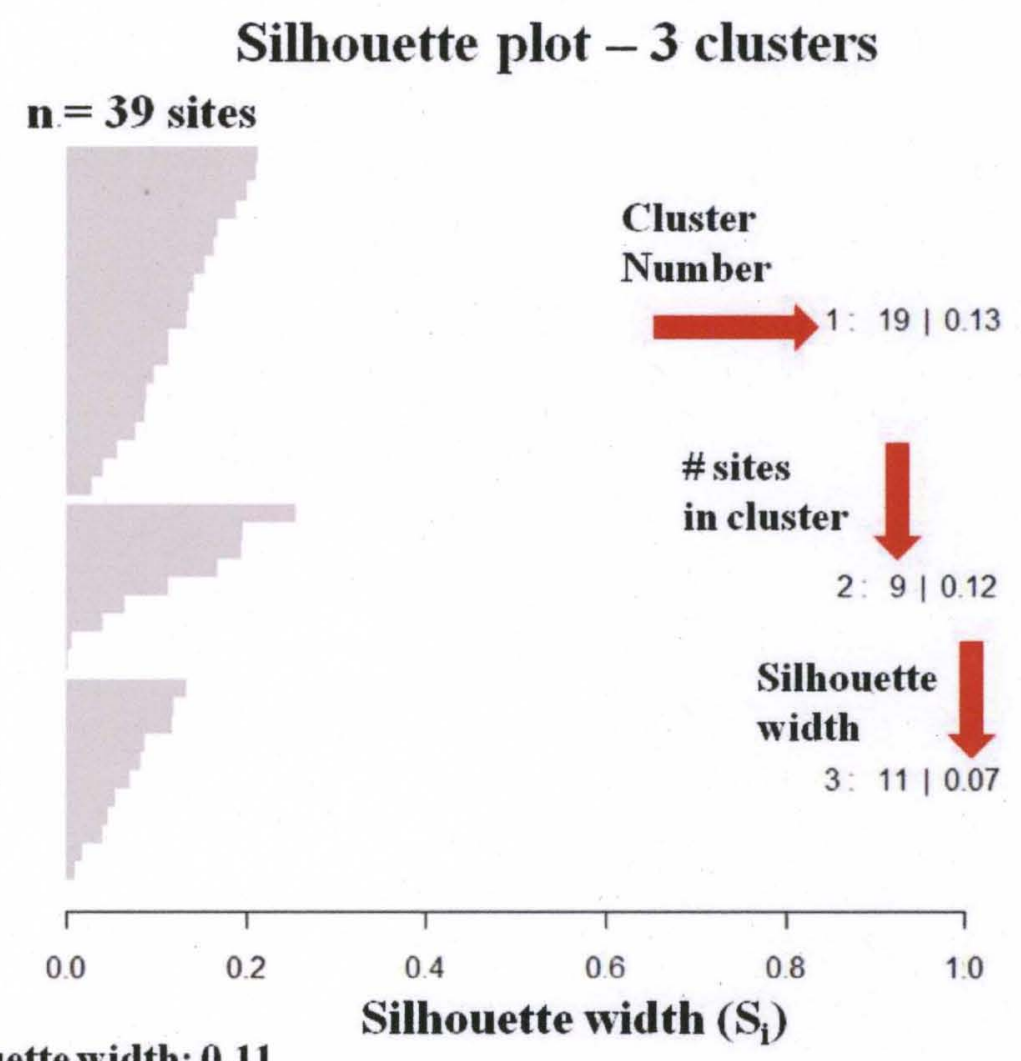

Overall silhouette width: 0.11

Figure 3-3: Silhouette plot of a cluster analysis (complete clustering using Bray-Curtis distance) resulting in three groupings of research sites. Sites within clusters are represented by gray bars, the length of each demonstrating the silhouette width (value on the $\mathrm{x}$-axis) of that particular site within the cluster. Cluster numbers, number of sites within the cluster, and cluster widths are to the right of each cluster. Overall silhouette widths are located below the numerical axis. Explanation of values given in the text. 


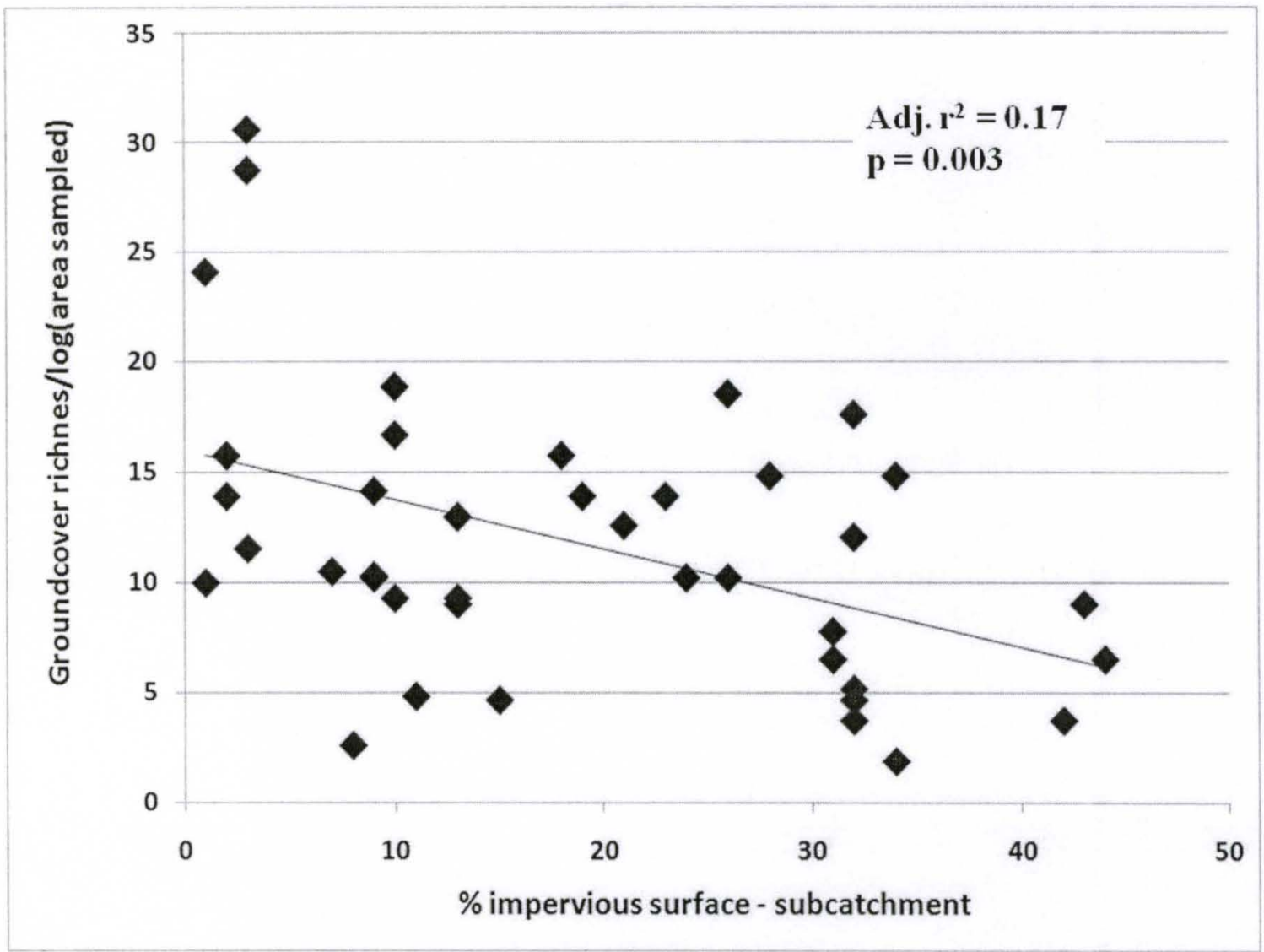

Figure 3-4: Groundcover species richness variation in nearstream plots with \% impervious surface at the subcatchment scale. Species richness (S) was first normalized prior to analysis as $\mathrm{S} / \log \left(\right.$ area sampled). The adjusted $\mathrm{r}^{2}=0.17$ and the $\mathrm{p}$-value $=0.003$. 


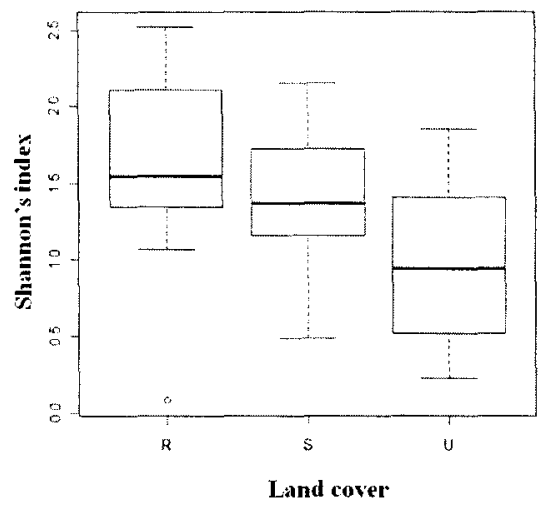

(a)

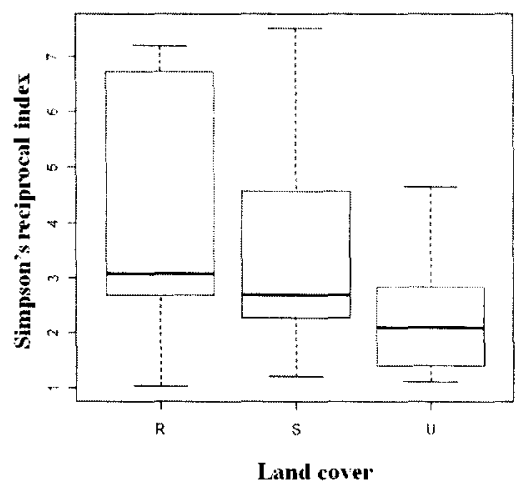

(b)

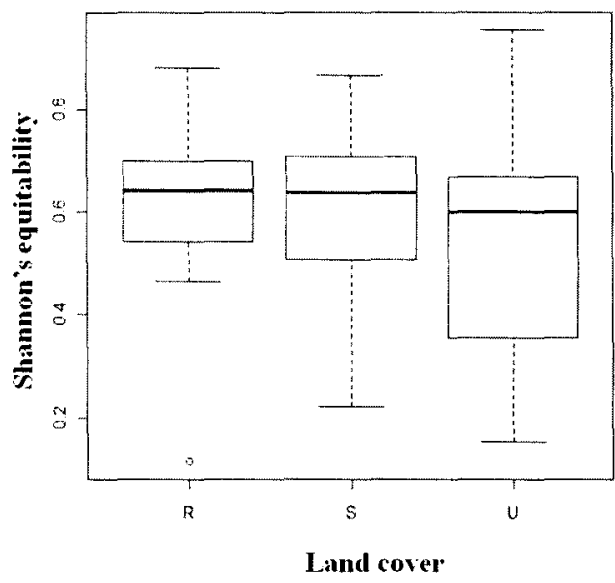

(c)

Figure 3-5a-c: Box-and-whisker diagrams of diversity indices computed for groundcover species in urban, suburban, and rural riparian zones. The bold line indicates the median, boxes delineate $25^{\text {th }}$ and $75^{\text {th }}$ percentiles, and whiskers indicate minimum and maximum values. (a) Shannon's, (b) Simpson's reciprocal, and (c) Shannon's equitability indexes were determined for all nearstream plots. Land use assignment for plots was determined at the subcatchment scale ( $U=U$ rban; $S=$ Suburban; $R=$ Rural). ANOVA's revealed a significant difference in Shannon's index (a) between land use categories $(p=0.023)$. Follow up analysis using Tukey's HSD pairwise comparisons revealed a higher Shannon's index in rural plots $($ mean $=1.61)$ than in urban plots $($ mean $=0.99 ; p=$ $0.020)$. Simpson's reciprocal index, while not exhibiting any significant differences $(p=$ 0.088 ), did show the same trend where rural plots had a higher mean index value (mean $=$ 3.88 ) than urban plots (mean $=2.35 ; \mathrm{p}=0.077$ ). Shannon's equitability index did not significantly differ among land use categories. Open circles represent potential outliers. 


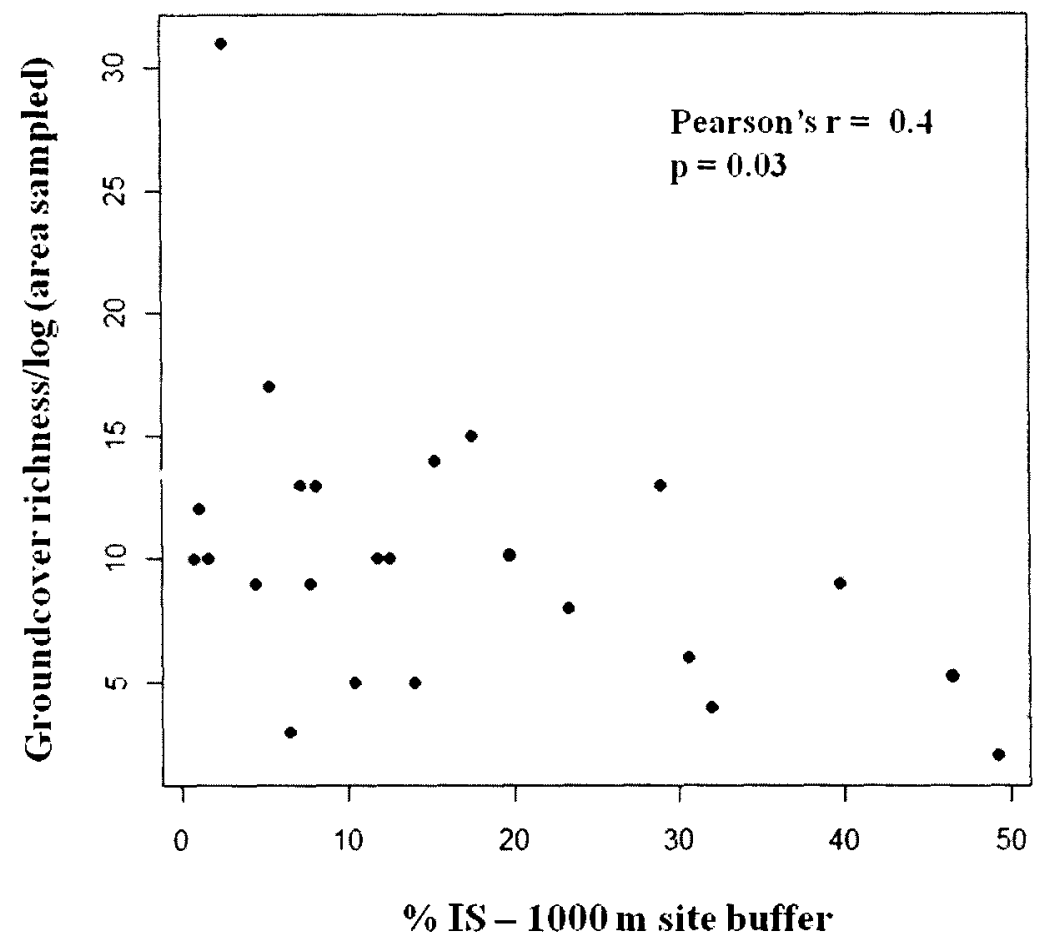

(a)

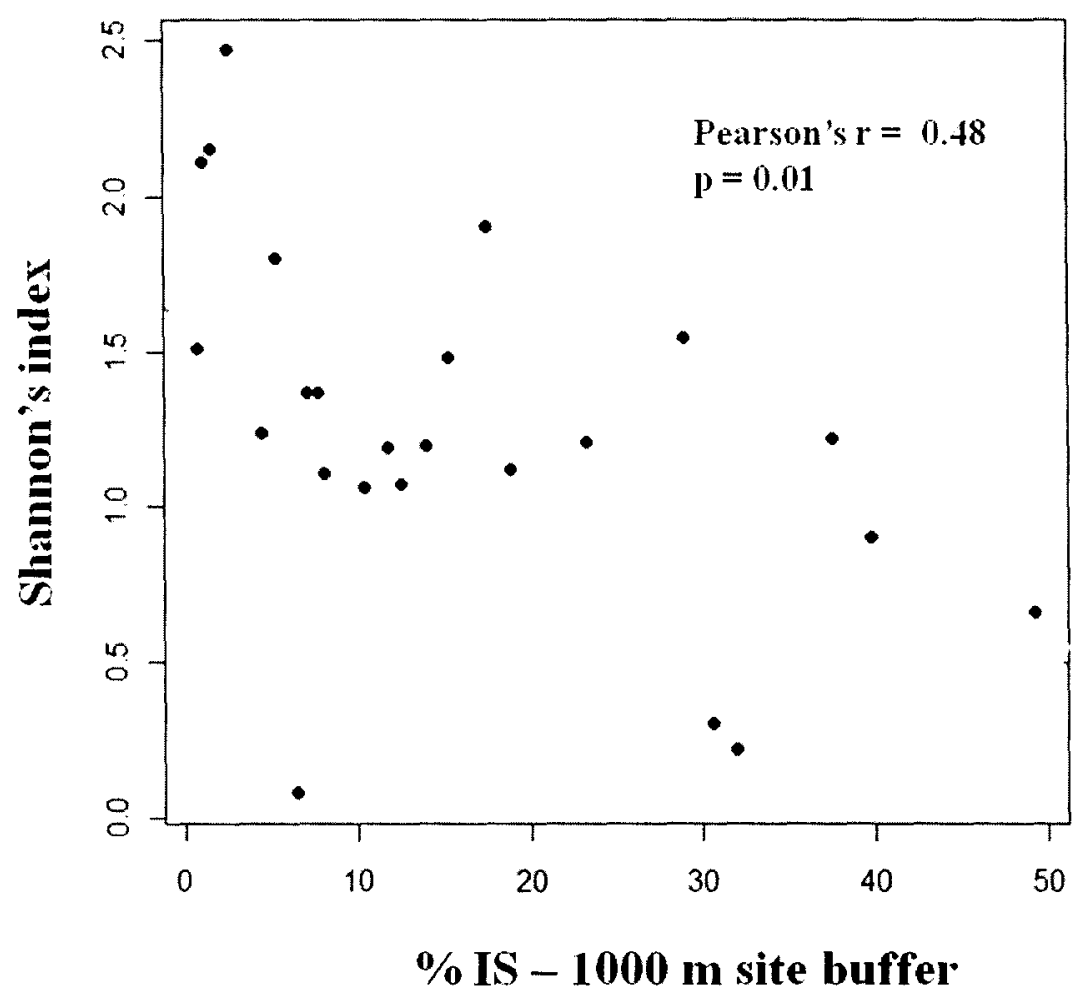

(b) 


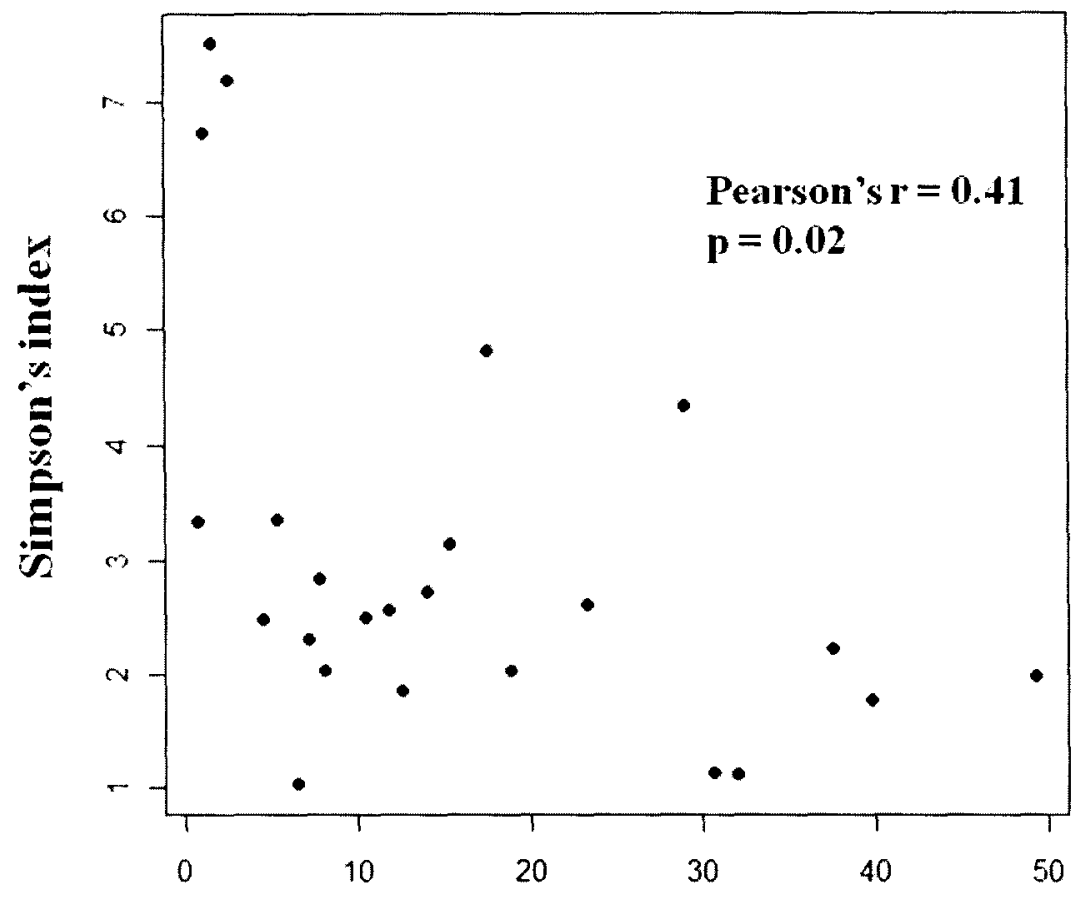

(c)

$\%$ IS - $1000 \mathrm{~m}$ site buffer

Figure 3-6a-c: Relationship between diversity measures of groundcover species at riparian plots along first order streams and \% impervious surface cover within 1000-m of each plot. Diversity measures shown are species richness (a), Shannons index (b), and Simpson's reciprocal index (c). Species richness (S) was normalized based on plot size as $\mathrm{S} / \log ($ area sampled) since nearstream plot sizes varied. 


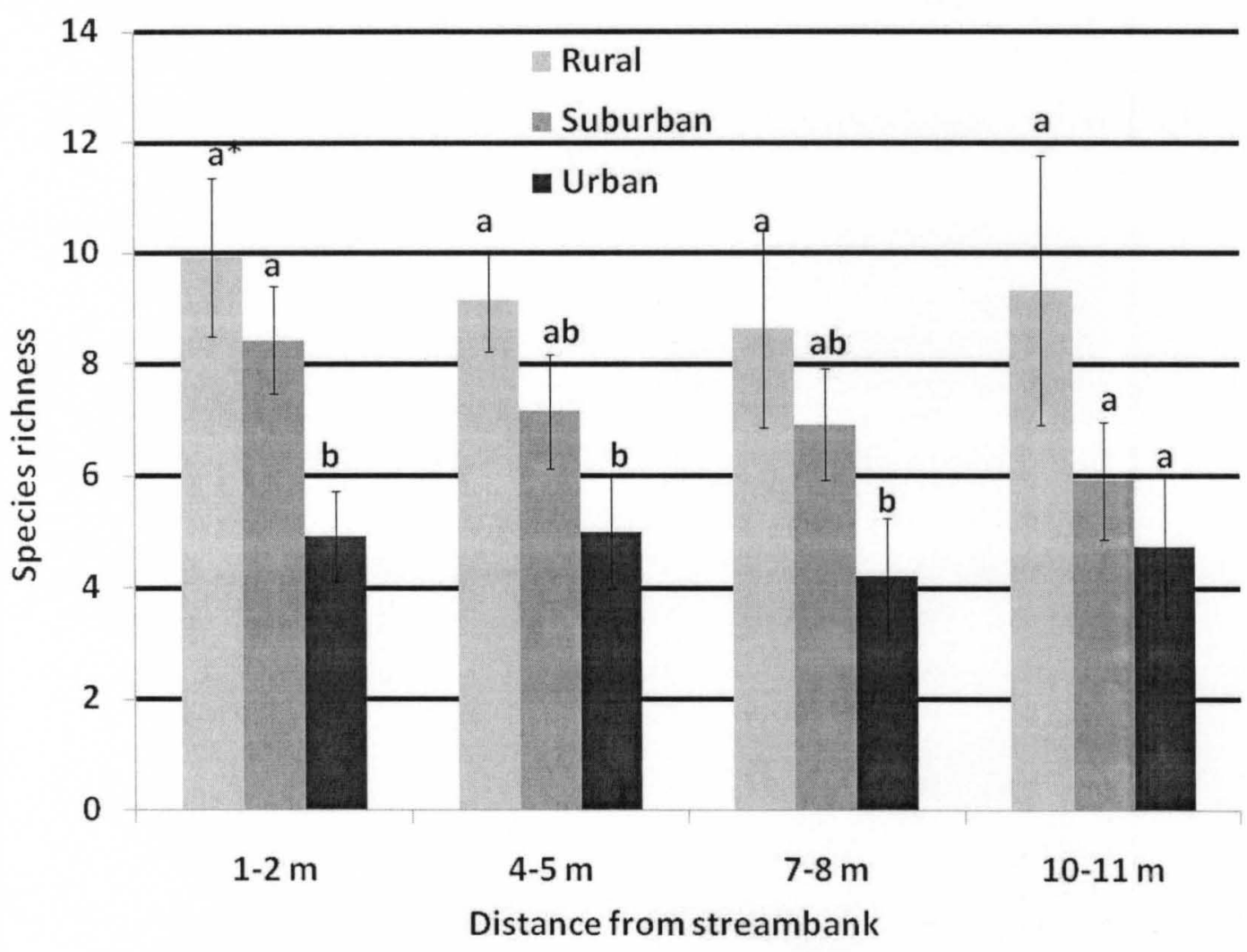

Figure 3-7: Mean groundcover riparian species richness at indicated distance intervals from the streambank in urban, suburban, and rural riparian zones. Land use assignment for plots was determined at the subcatchment scale. Data were obtained from plots nearest the stream. Each distance interval at each plot was composed of three $1 \times 1 \mathrm{~m}^{2}$ quadrats. Species richness was averaged by distance interval for each land use category. Differing letters above the standard error bars indicate significant differences at $\alpha=0.01$ * or 0.05 . Richness values were natural $\log$ transformed for the $1-2$ and $4-5-\mathrm{m}$ intervals prior to analysis to meet the assumptions of ANOVA, although raw mean species richness values were used to create graphics. 


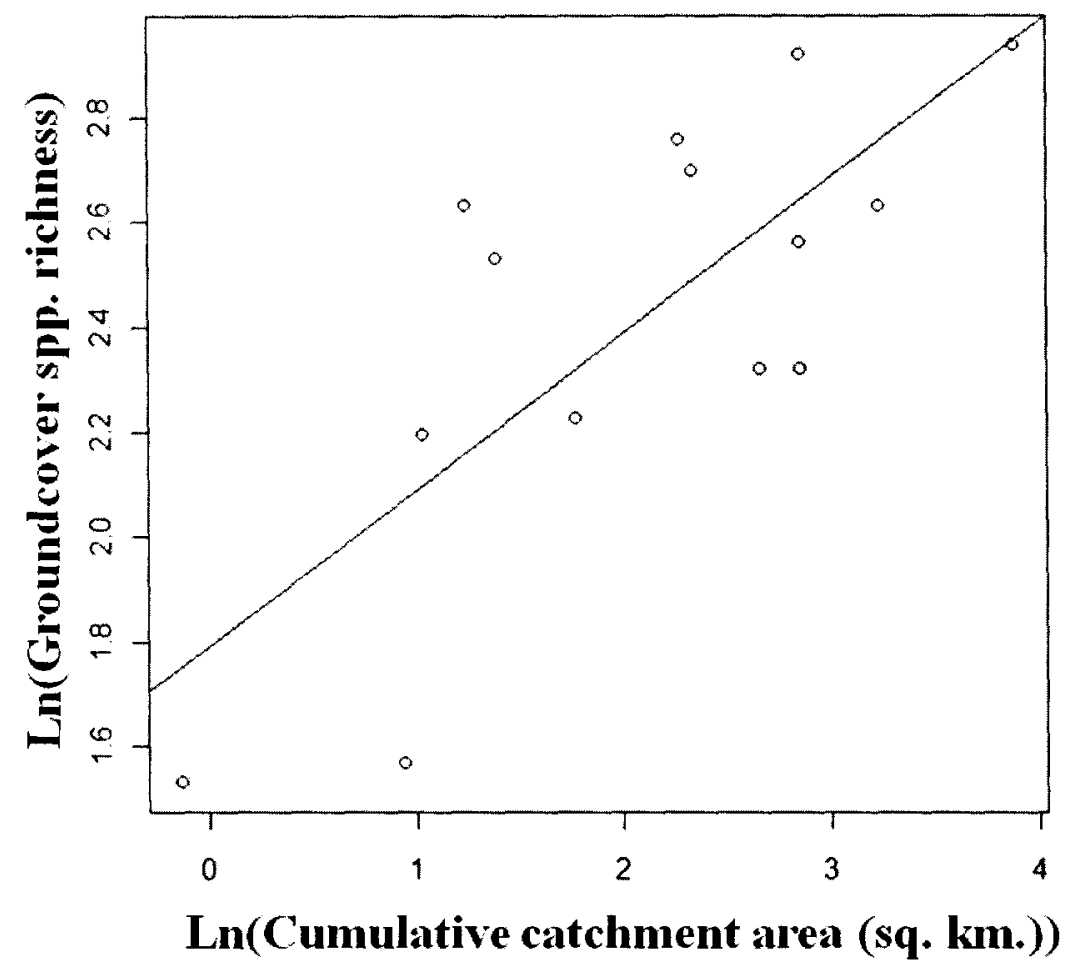

Figure 3-8: Linear regression model of cumulative catchment area upstream of the subcatchment for each suburban nearstream plot against normalized groundcover species richness $(\mathrm{S} / \log ($ area sampled $))$. The adjusted $\mathrm{r}^{2}=0.54$, and the $\mathrm{p}$-value $=0.0011$. 


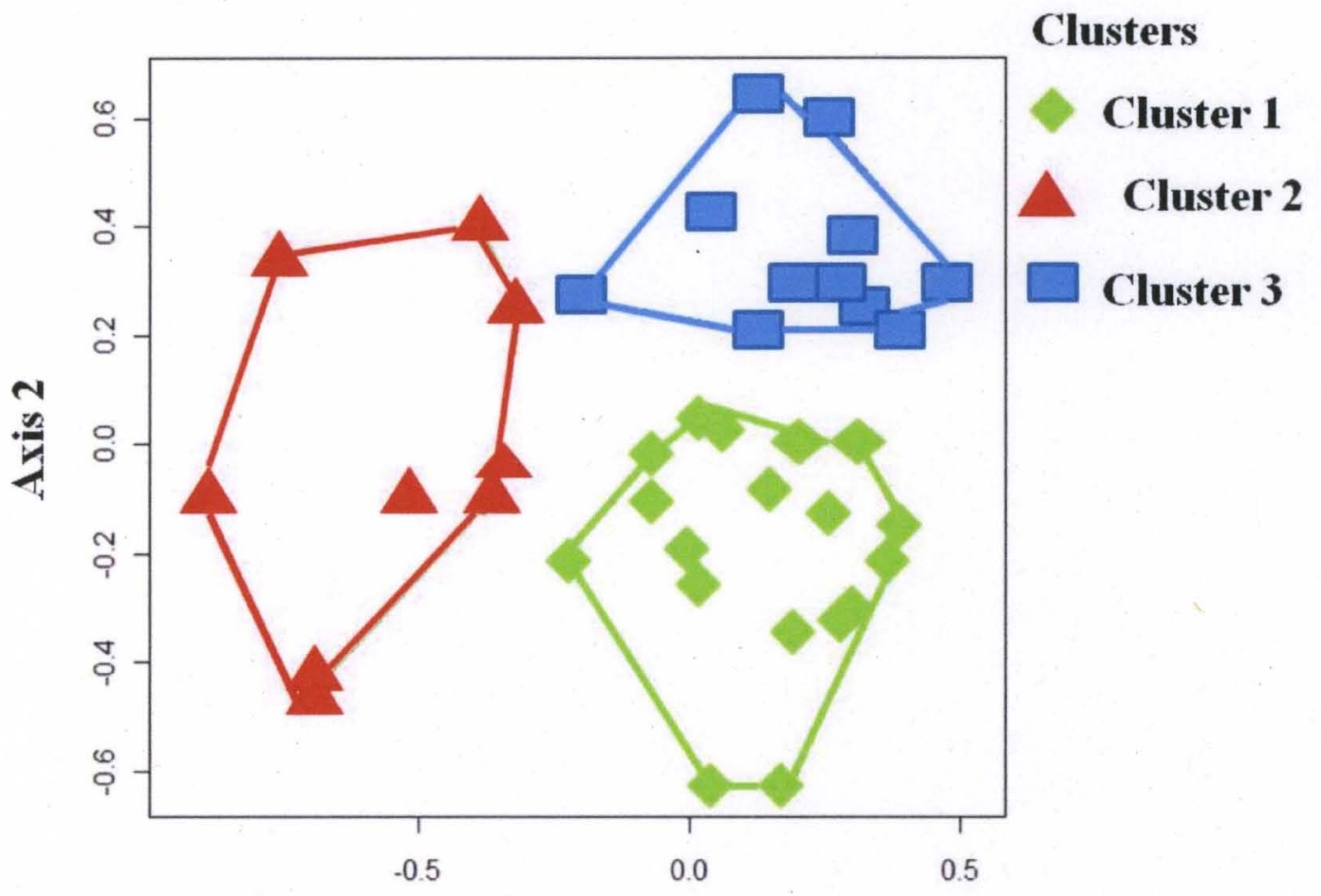

\section{Axis 1}

Figure 3-9: Non-metric multidimensional scaling (NMS) 3-dimensional configuration of research sites arranged by groundcover species composition. Research sites (points) assigned to their respective groupings from the 3-cluster configuration from cluster analysis. Grouped clusters are shown along axes $1 \& 2$. 


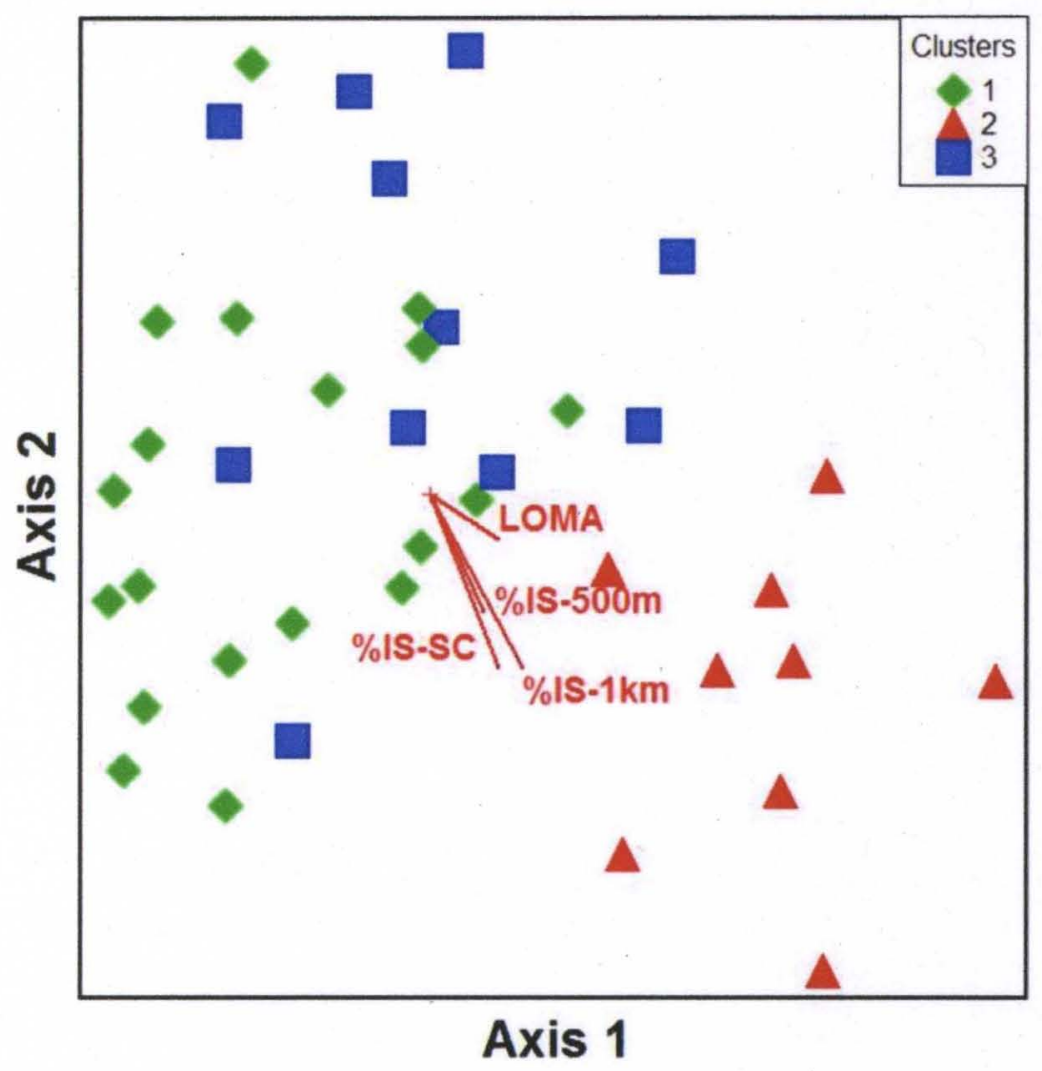

(a)

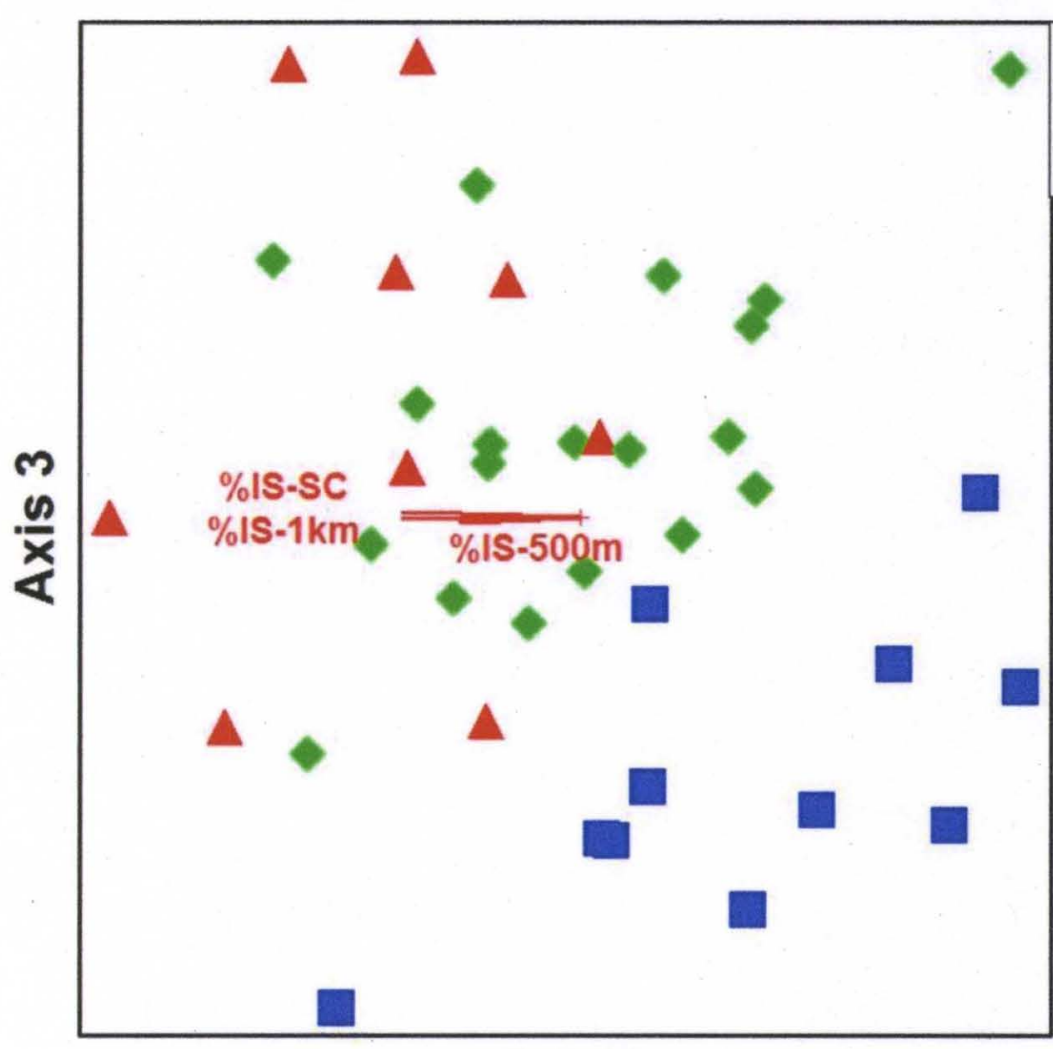

Axis 2

(b) 


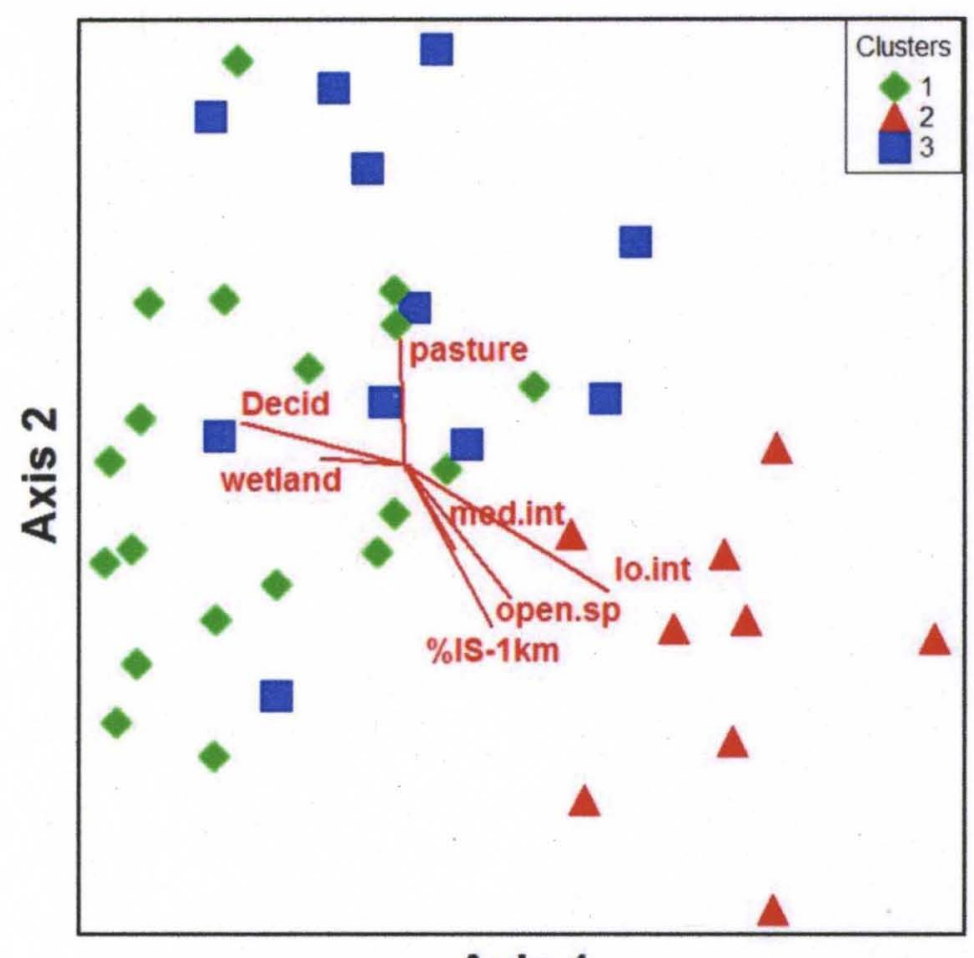

Axis 1

(c)

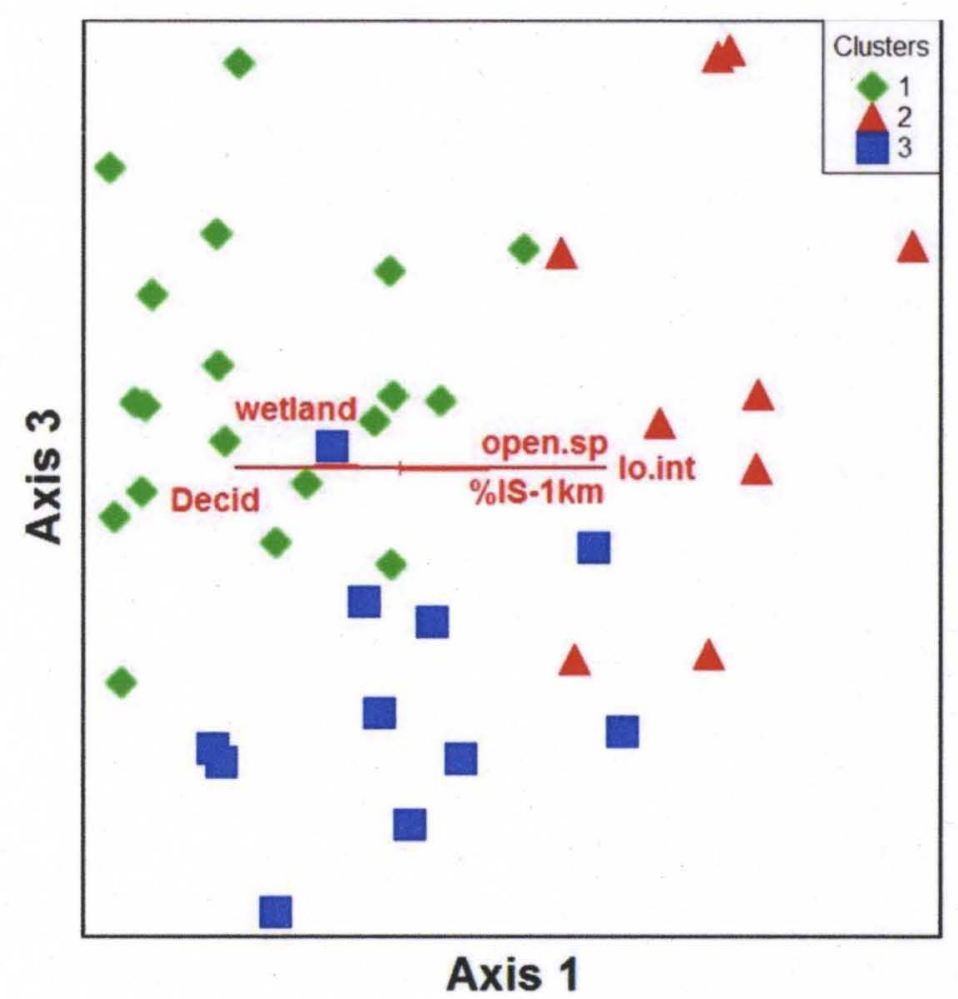

(d) 
Figure 3-10a-d: Non-metric multidimensional scaling (NMS) 3-dimensional configuration of research sites arranged by groundcover species composition and colorcoded by cluster assignments from the 3 -cluster configuration using cluster analysis. Cluster identities are identified in the legend. Vectors representing site environmental metrics (created using the biplot feature in PC-Ord (McCune and Mefford 1999)) are pointing in the direction of increasing proportions, with the length of each vector representing the strength of correlation. Vectors for environmental metrics are shown only if they have an $r^{2} \geq 0.15$ along at least one axis. Environmental metrics shown along (a) axes $1 \& 2$ and (b) axes $2 \& 3$ are the proportion of impervious surface (\%IS) surrounding each site at 3 scales: subcatchment (SC), $1-\mathrm{km}$ and $500-\mathrm{m}$ radius from the site center. Since \%IS at the 1-km scale exhibited the strongest correlation with the site arrangement ( $\mathrm{a} \& \mathrm{~b}$ ), I chose to use environmental metrics from the National Land Cover Database. Environmental metrics are shown along the same axes in (c) and (d) include low- (lo.int) and medium- intensity (med.int) developed, open-space developed (open.sp), $\%$ IS within a $1-\mathrm{km}$ site radius (\%IS- $1 \mathrm{~km}$ ), pasture, and deciduous forest (Decid). Definitions are included in Appendix Table 3. Explained axis variance $\left(\mathrm{r}^{2}\right)$ is as follows: Axis $1-0.25$, Axis $2-0.22$, Axis $3-0.25$. 


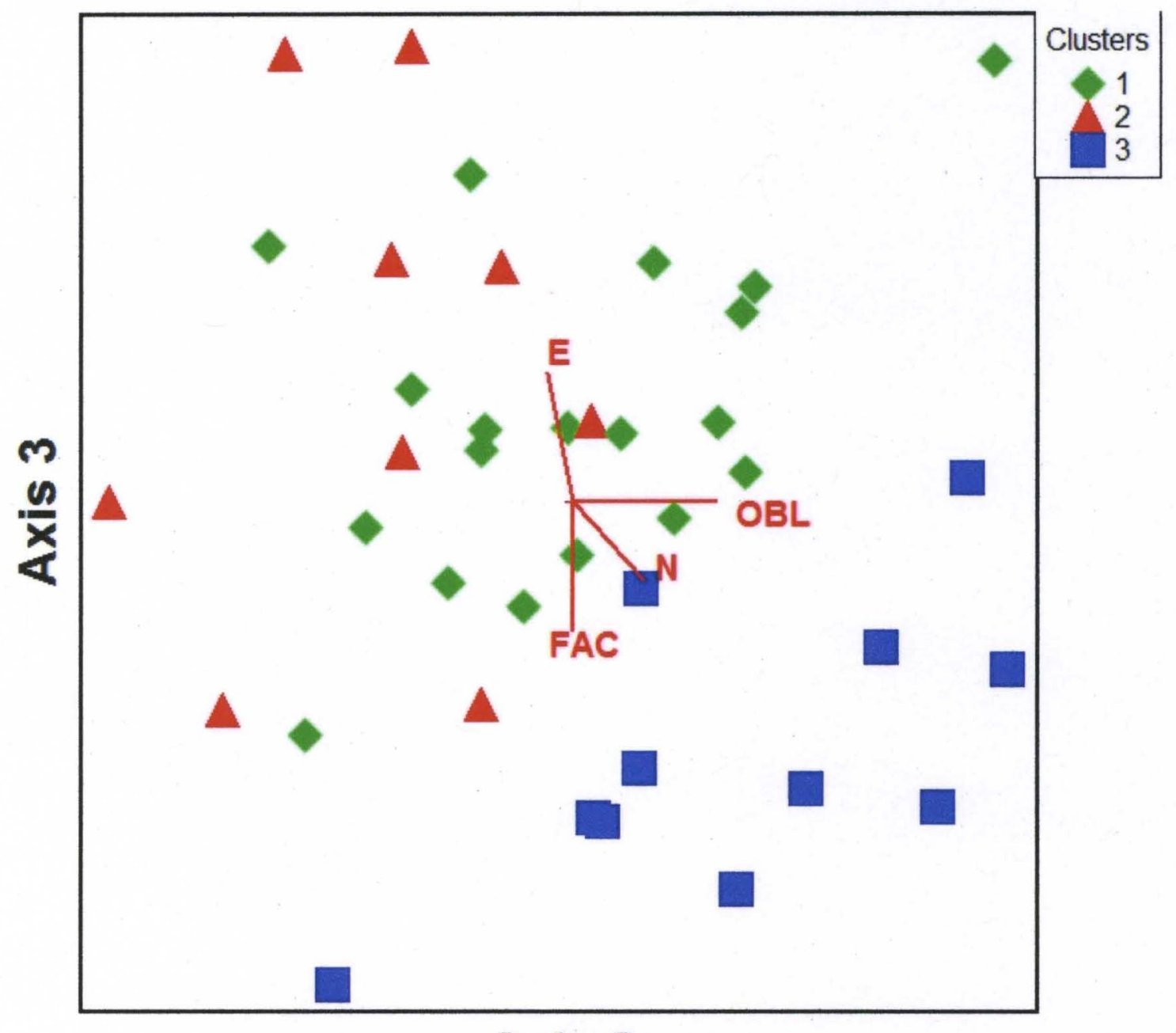

Axis 2

Figure 3-11: Non-metric multidimensional scaling of research sites arranged according to groundcover species composition. Sites are color coded according to their cluster assignments from the 3-cluster configuration using cluster analysis. Cluster identities are identified in the legend. Vectors representing species attributes (created using the biplot feature in PC-Ord (McCune and Mefford 1999)) are pointing in the direction of increasing importance values, with the length of each vector representing the strength of correlation. Vectors for species attributes are shown only if they have an $r^{2} \geq 0.15$ along at least one axis. The species attributes shown are facultative (FAC), obligate wetland $(\mathrm{OBL})$, native $(\mathrm{N})$ and exotic $(\mathrm{E})$ species. Definitions and the full list of attributes included in the analysis are in Appendix Table 3. As a 3-dimensional view is not an option using the biplot feature, separation of sites are shown only along axes 2 and 3 to enable better visualization of the relationship between environmental variables and cluster categories in two dimensions. Explained axis variance $\left(\mathrm{r}^{2}\right)$ is as follows: Axis $2-$ 0.22 , Axis $3-0.25$. 


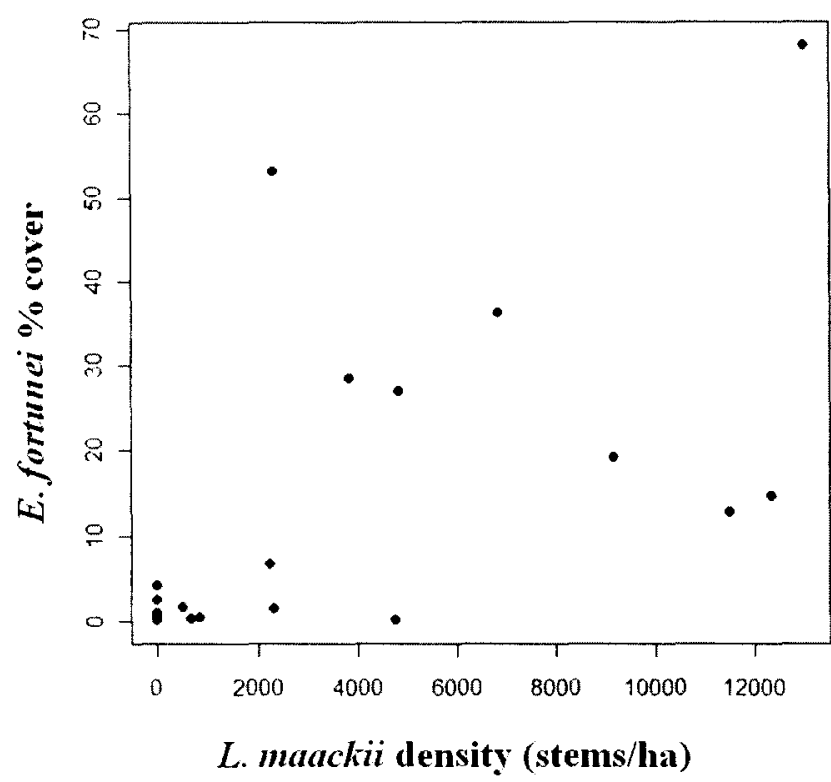

(a)

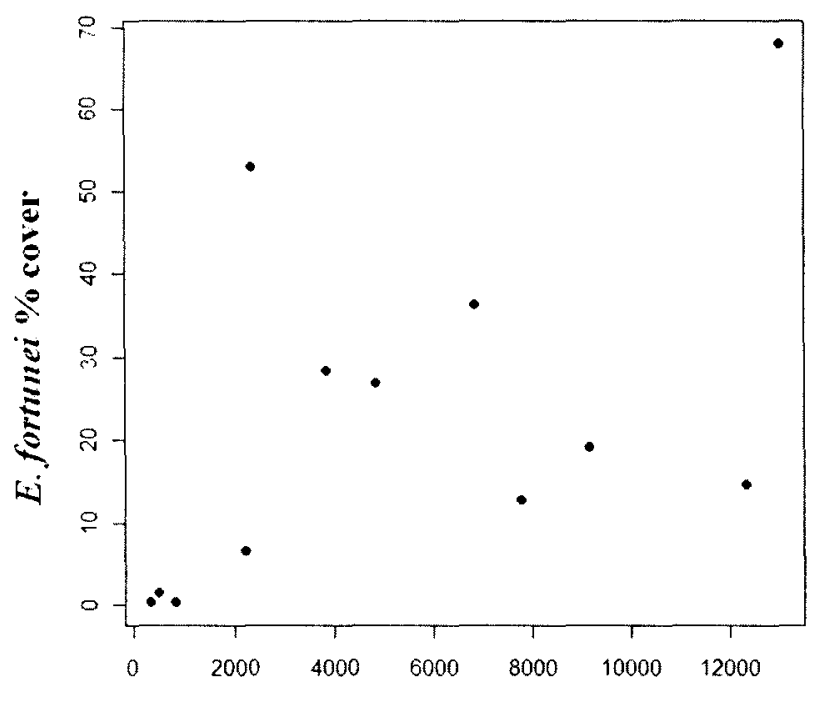

L. maackii density (stems/ha)

(b)

Figure 3-12a-b: Scatterplots of Euonymus fortunei percent cover in response to Lonicera maackii density. All nearstream plots were included in the regression that contained $E$. fortunei (a), or that contained both E. fortunei and L. maackii (b). Data were square root (a) or natural $\log$ (b) transformed prior to regression to better meet statistical assumptions. One outlier was removed from graph (a), and two were removed from graph (b) following outlier analysis to better meet statistical assumptions. Pearson's $r$ and a $p$ values were (a) Pearson's $r=0.73$ and $p=0.0001$, and (b) Pearson's $r=0.81$ and $p=$ 0.001 . 


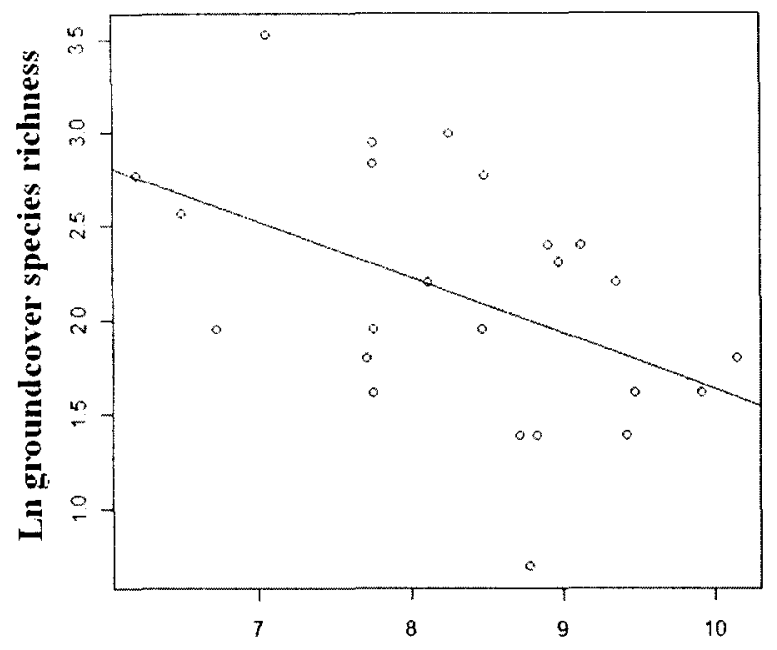

Ln L. maackii density (stems/ha)

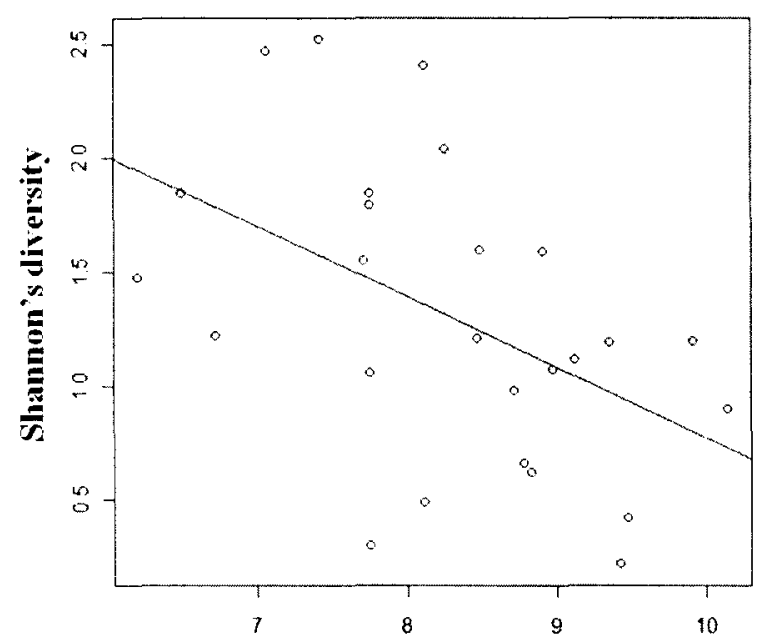

Ln L. maackii density (stems/ha) (a)

(b)

Figure 3-13a-b: Species richness (a) and Shannon's diversity (b) of groundcover species in nearstream plots in response to Lonicera maackii density. Species richness and $L$. maackii density was natural log transformed prior to regression in (a). Only plots that contained L. maackii were included. Two outliers were removed from graphic (a) following outlier analysis to better meet statistical assumptions. The adjusted coefficient's of determination and $p$-values were (a) $r^{2}=0.19$ and $p=0.019$, and (b) $r^{2}=$ 0.20 and $p=0.012$. 


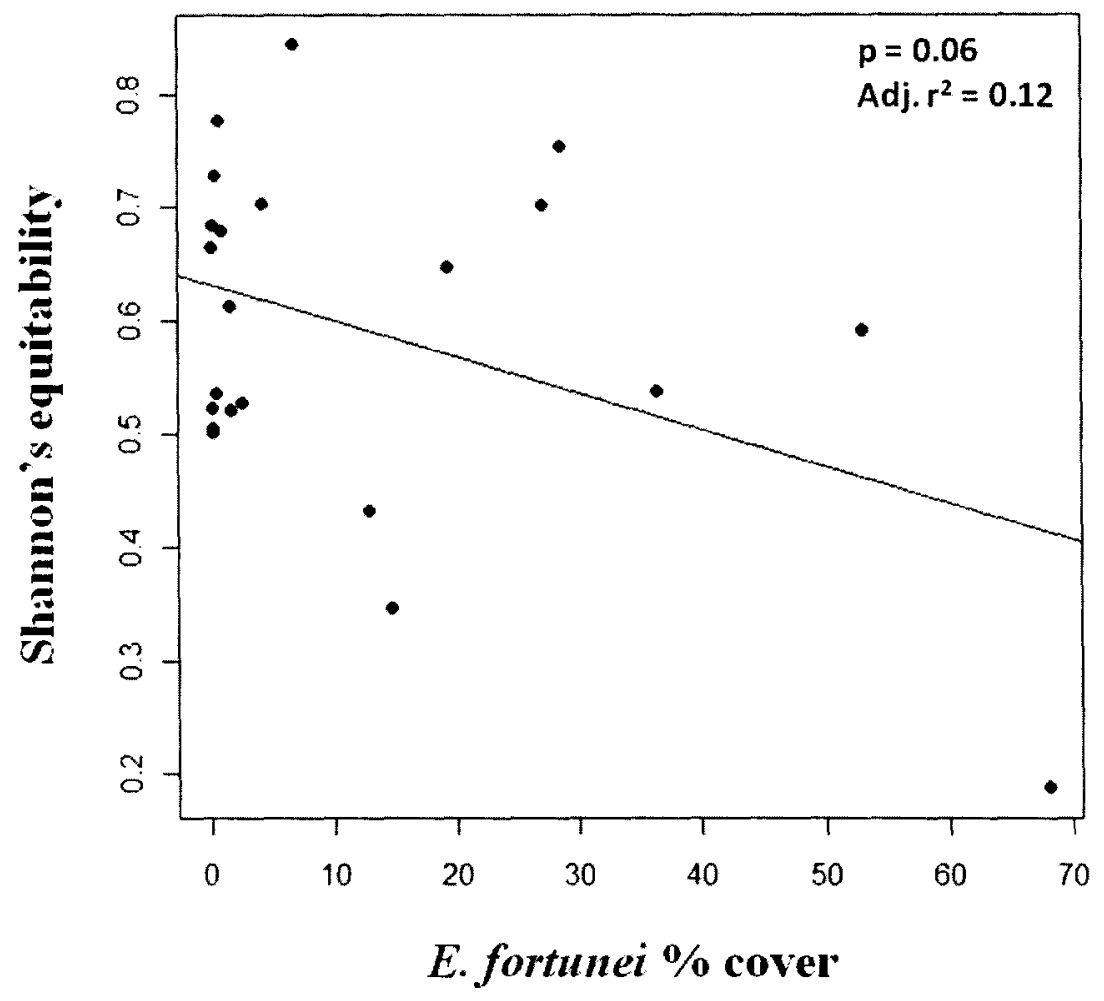

Figure 3-14: Shannon's equitability of groundcover species in nearstream plots in response to Euonymus fortunei percent cover. Only plots that contained $E$. fortunei were included. The adjusted coefficient of determination was $\mathrm{r}^{2}=0.12$, and the $\mathrm{p}$-value was $\mathrm{p}$ $=0.06$. 


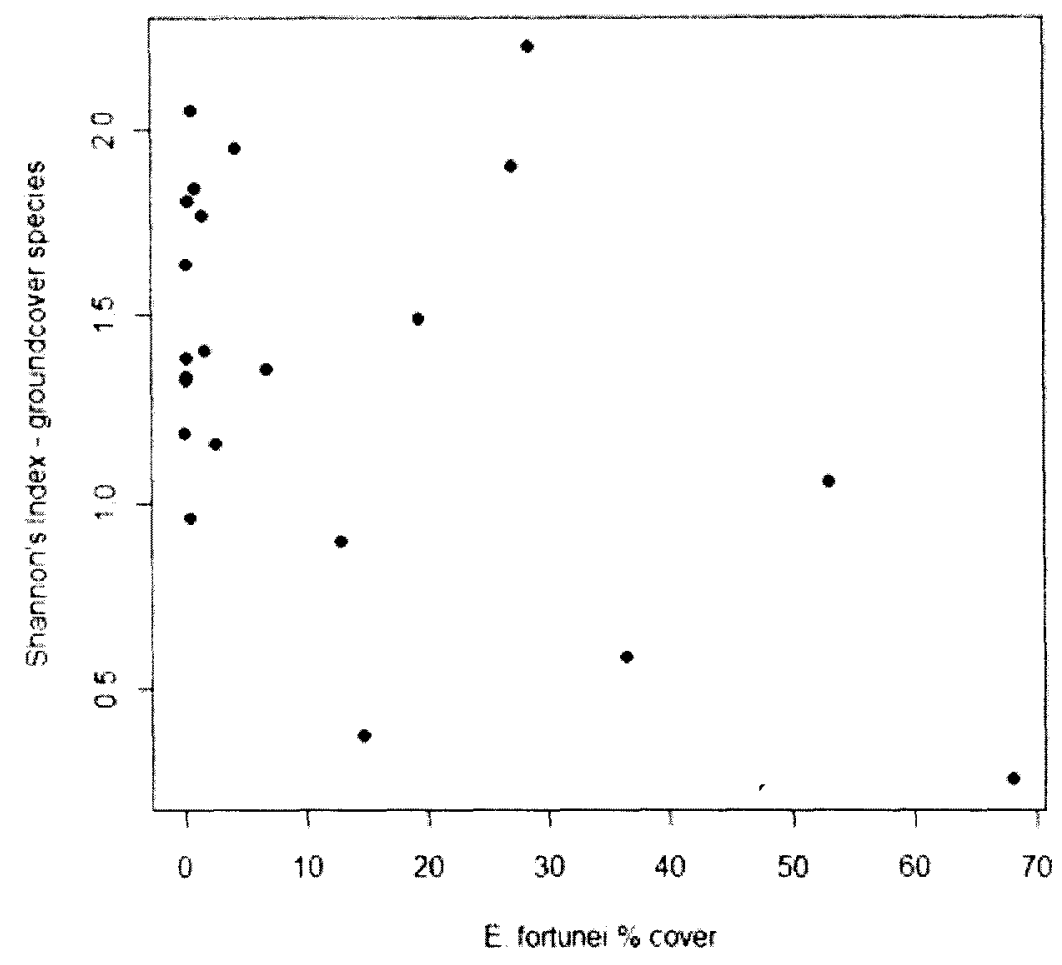

(a)

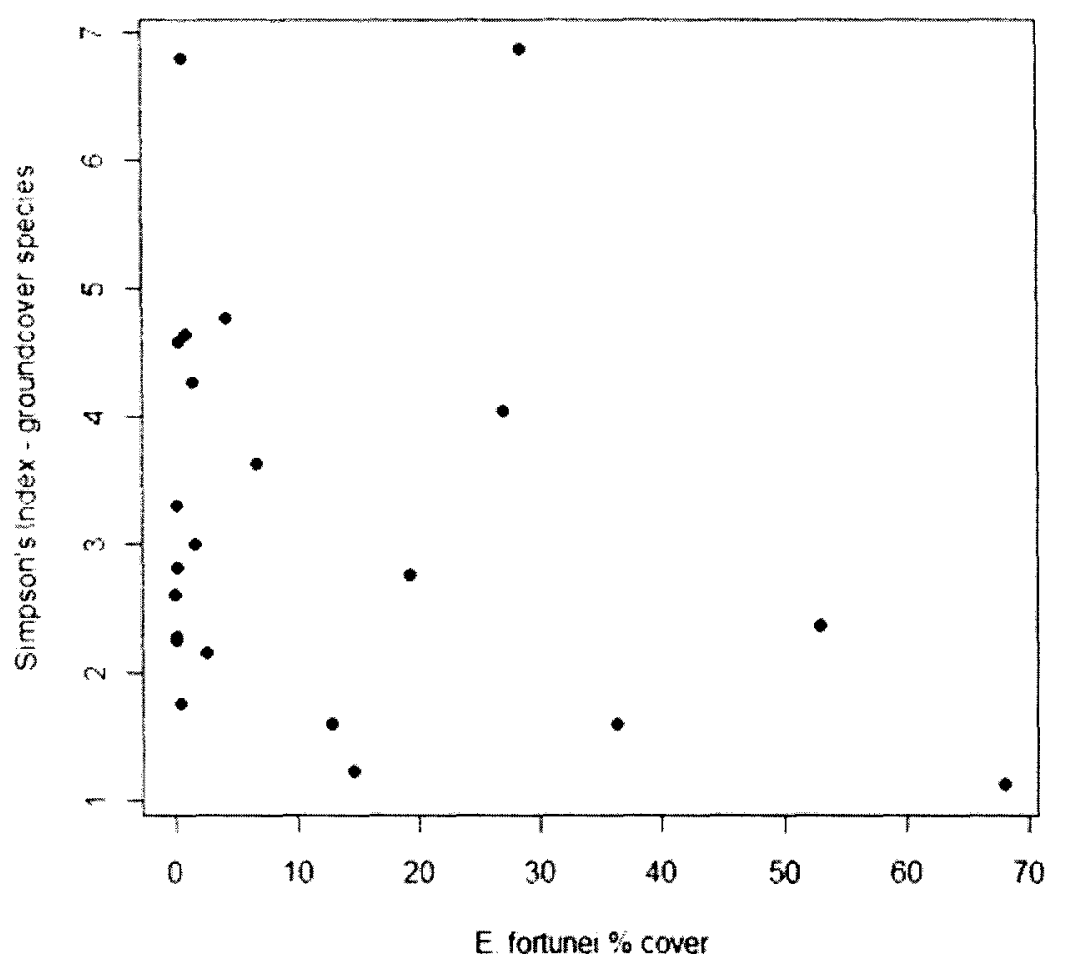

(b) 


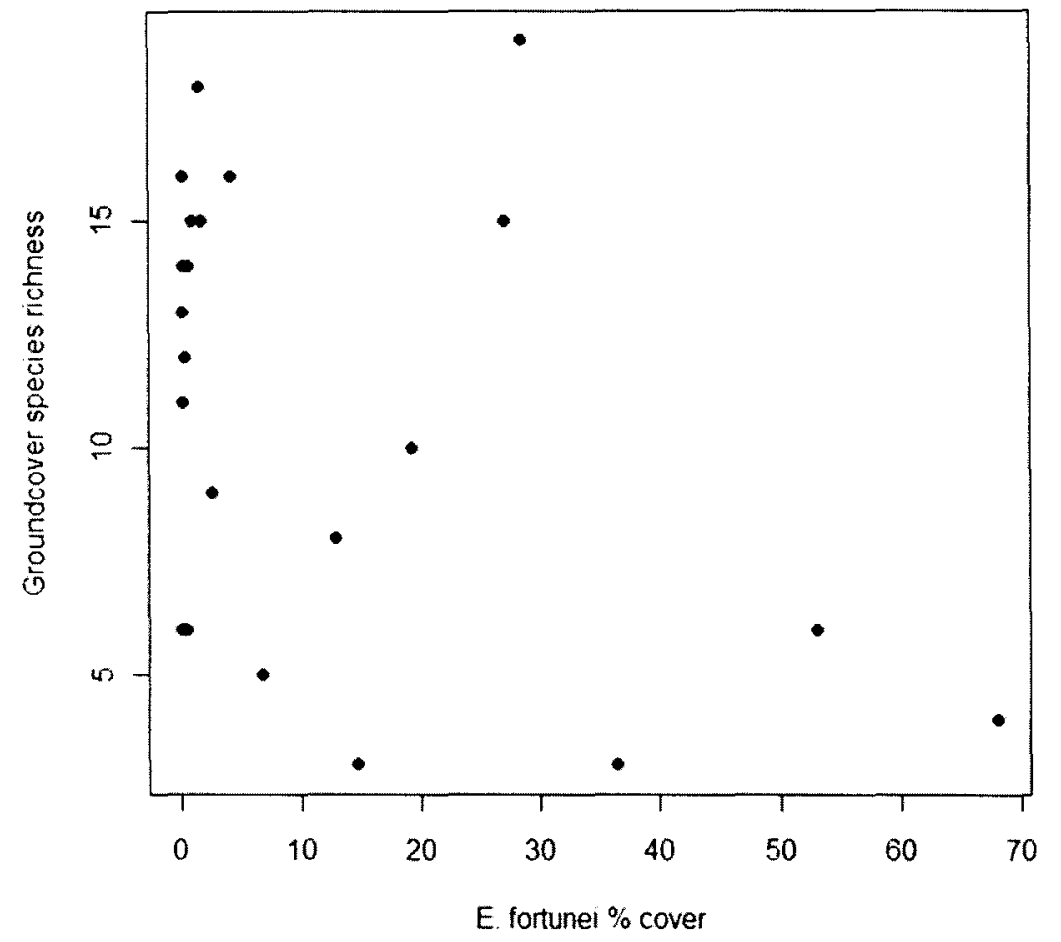

(c)

Figure 3-15a-c: Shannon's Index (a), Simpson's Index (b), and species richness (c) of groundcover species in nearstream plots in response to Euonymus fortunei percent cover. Only plots that contained $E$. fortunei were included. 


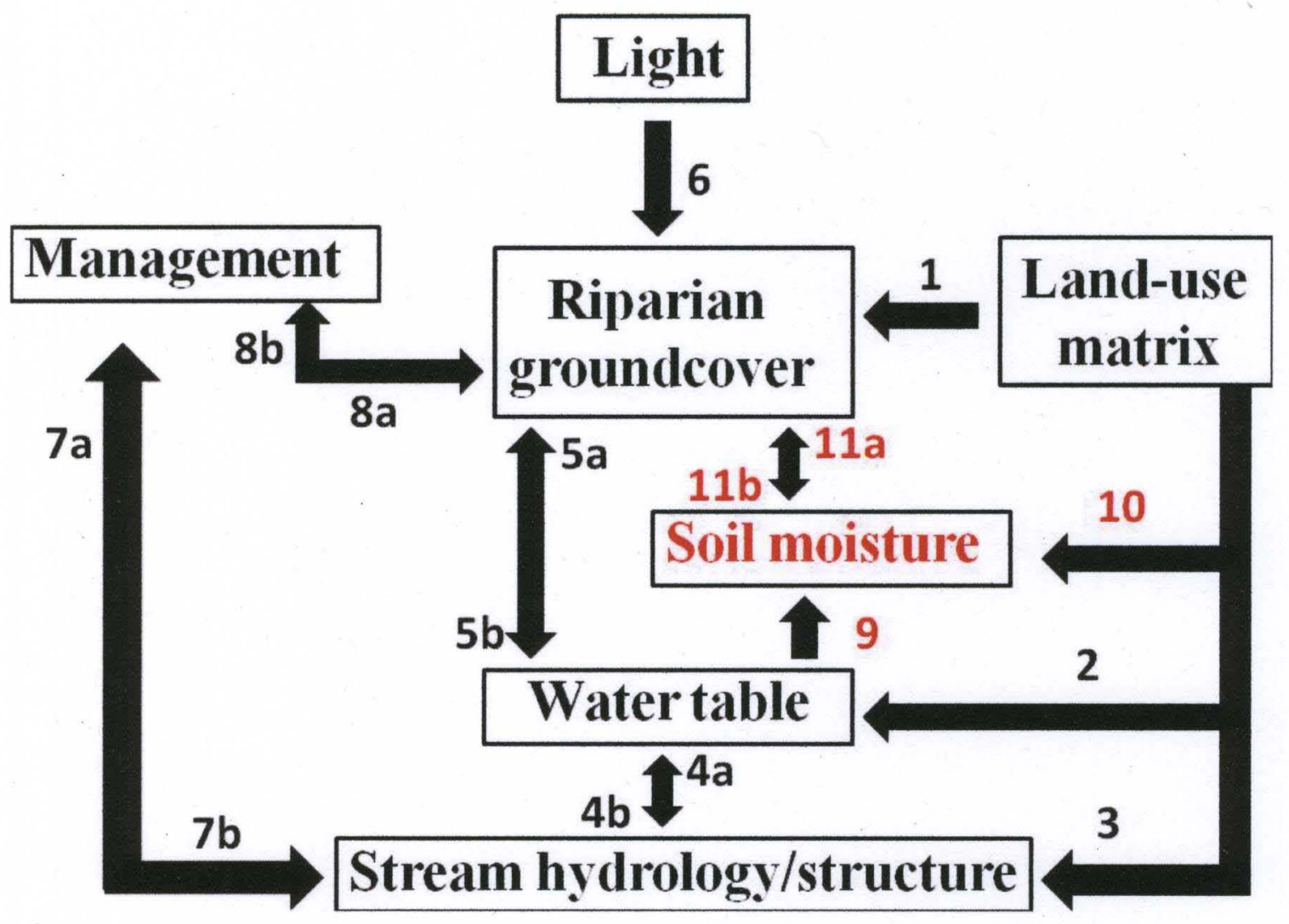

Figure 3-16: New conceptual model of variables that affect and are affected by vegetation within a riparian area modified from Figure 1. Additional arrows in need of further study are depicted in red. A direct link between groundwater levels and riparian vegetation was not found. Soil moisture was added as an intermediary to refocus attention on that link to the vegetation composition within riparian zones. The water table can still limit species composition if it is close enough to the soil surface (Arrow 5a). The water table along with surrounding land-use can also affect soil moisture (Arrow $9 \& 10$ respectively). Where species are not limited by saturated soil, soil moisture may play a larger role in species composition by favoring species that are better able to compete under existing moisture conditions (Arrow 11a). Riparian groundcover can feedback on soil moisture depending on proportion of the soil that is shaded by leaf cover and the transpiration rates of various plants (Arrow 11b). Descriptions of other arrows as in Figure 1-1. 


\title{
CHAPTER 4: SEASONAL GREENHOUSE GAS FLUX IN RIPARIAN SOILS
}

\author{
ALONG URBAN, SUBURBAN, AND RURAL RIPARIAN ZONES IN METRO \\ LOUISVILLE, KENTUCKY
}

\section{Introduction}

Removal of nutrients before they reach the stream is a major function of the vegetation, soils and soil microbes in riparian zones (Lowrance et al. 1997, Groffman et al. 2003). In riparian zones the pathways and microbial transformations of nutrients are significantly controlled by the presence or absence of oxygen. Since the water table is closer to the surface in riparian areas than in upland areas, variation in depth to water table can affect the balance of aerobic vs. anaerobic microorganisms and gas transport from the soil to the atmosphere and vice versa. Under water-saturated soil conditions that occur in soils of forested riparian sites, aerobic microorganisms require elemental $\mathrm{O}_{2}$ as the final electron acceptor during respiration and, therefore, cannot thrive under high soil moisture conditions of long duration. In contrast, anaerobically respiring microorganisms can use compounds other than $\mathrm{O}_{2}$ for this process, and therefore flourish under low to no oxygen conditions.

Understanding how landscape level variation controls nutrient transformations within riparian zones is important, since they lie adjacent to bodies of water and filter and transform nutrients entering these aquatic systems. Riparian zones have been shown to be effective in removing sediment (Cooper et al. 1987, Lowrance et al. 1988), ammonium $\left(\mathrm{NH}_{4}{ }^{+}\right)\left(\right.$Jacobs and Gilliam 1985a, Puckett 2004), nitrogenous compounds $\left(\mathrm{NO}_{3}{ }^{-}\right)$, and $\mathrm{P}$ 
(Cooper et al. 1987). Eutrophication of water bodies in urban and suburban areas is common and of concern to state and regulatory agencies that are responsible for water quality (Kolenbrander 1972, Gilliam 1994, Groffman et al. 2002). Nitrogen inputs to streams have been shown to occur to a greater extent from urban riparian areas than from forested reference riparian areas (Groffman et al. 2004). Studying the effects of urbanization on the soil processes that remove or supply these nutrients to water bodies is a worthwhile endeavor from both economic and water quality standpoints.

Gas effluxes $\left(\mathrm{CO}_{2}, \mathrm{CH}_{4}, \mathrm{~N}_{2}, \mathrm{~N}_{2} \mathrm{O}\right)$ from soils are among the routes by which carbon and nitrogen inputs into streams are decreased. As compared to forested rural catchments, soil gas fluxes may differ within urban and suburban catchments via several mechanisms (Raich and Schlesinger 1992, Groffman et al. 2004, Groffman et al. 2006). Riparian soils along urban-to-rural gradients may differ in the quality and quantity of organic matter, inorganic nutrients, bulk density, soil moisture and depth to water table, all of which affect nutrient cycling rates and directions. In addition to variation in parent soil material, such urban-rural differences are dependent on the width of the forested buffer. Forests within ten meters of edges were found be efficient traps for nutrients when compared to 200-m towards the forest interior (Draaijers et al. 1988). Degree of riparian colonization by different species of woody plants would also affect riparian nutrient sink functions. Species differences in growth rate, size, phenology, and morphology affects net primary productivity, which in turn affects the quantity and quality of carbon inputs (Ehrenfeld 2003) and nutrient uptake dynamics in riparian systems. For example, such changes in urban and suburban plant communities could be due to exotic plant invasion. Urban and suburban riparian areas are highly susceptible to exotic species invasions 
(Burton et al. 2005, Burton and Samuelson 2008) due to proximity of exotic seed sources, management planting and disturbances, as well as altered stream hydrology. Variation in atmospheric nutrient deposition could also potentially affect nutrient cycling differentially along urban-to-rural gradients via direct inputs to soils and by affecting plant productivity and community composition. Bulk density differences can influence the anaerobic or aerobic nature of the soil. High bulk density results in more anoxic soils (Ball 1999). Stream channelization can cause more rapid losses of ground water and decreased soil moisture in urban riparian areas (Groffman et al. 2003). These factors alter soil oxygen concentrations, microbiological communities, and atmospheric-soil gaseous exchange rates.

In addition, since urban areas tend to be hotter than their rural counterparts due to the urban heat island effect. Given similar canopy coverage, riparian gas flux processes could also differ between urban and rural areas due to higher soil temperatures increasing the metabolic activity of plant roots and microbes. Higher atmospheric temperatures in urban areas can also potentially result in drier soils by accelerating evapotranspiration. Also, higher streambanks along piedmont streams coupled with lower water tables in urban areas (see Dissertation Chapter 2) (Groffman et al. 2003) exacerbate soil drying, because upper soil layers are not inundated as frequently with water. As cities expand and become more populous, understanding source-sink dynamics of trace gas fluxes in riparian areas and the soil properties that drive them will be crucial to modeling greenhouse gas accumulation within the atmosphere.

This study's first objective, therefore, was to measure the fluxes of three greenhouse gasses $\left(\mathrm{CO}_{2}, \mathrm{CH}_{4}, \mathrm{~N}_{2} \mathrm{O}\right)$ over three seasons of the year along streams located 
in urban, suburban and rural catchments in and near the city of Louisville, Kentucky to determine if they varied predictably with increasing impervious surface area in these catchments. I predicted that $\mathrm{CO}_{2}$ flux, due to aerobic respiration by roots and microbes, would be higher in urban riparian areas due to dryer soils and potentially warmer temperatures in urban than in suburban or rural riparian habitats. I also predicted that net methane uptake into soils via methanotrophy would be greatest within urban riparian sites since methanotrophs are obligate aerobes. Conversely, I predicted a net methane efflux (methanogenesis) from soils in rural forested sites, because the anoxic environment created by shallower water tables should favor methanogenic over methanotrophic activity. Predicting land-use relationships with $\mathrm{N}_{2} \mathrm{O}$ flux is less straightforward, because it is the product of two processes, one aerobic (a by-product of nitrification), and the other anaerobic (denitrification).

The second objective was to determine if the urban, suburban and rural variation in flux rates could be related to variation in riparian characteristics expected to affect these rates. These included streambank height, depth to water table, and degree of plant community invasion by an exotic shrub, Lonicera maackii. I predicted that urban riparian water tables would be lower when compared to suburban and rural reference riparian sites because of higher urban streambanks, which I had demonstrated previously (see Dissertation Chapter 2). In addition, I reported in Chapter 2 (this dissertation) that urban and suburban riparian areas were densely colonized by the exotic shrub, L. maackii. I expected that this shrub could affect $\mathrm{N}_{2} \mathrm{O}$ gas fluxes at some times of the year due to its highly nitrogenous litter, which mineralizes $\mathrm{N}$ rapidly (Trammell 2010). Litters with rapid mineralization often stimulate soil nitrification, providing substrate to support 
denitrification under high moisture conditions. In addition, high soil nitrification rates would also lead to high rates of $\mathrm{N}_{2} \mathrm{O}$ emissions under drier, aerobic conditions, as well.

The third objective was to evaluate whether greenhouse gas fluxes, particularly $\mathrm{N}_{2} \mathrm{O}$, could be related to the concentrations of dissolved organic carbon (DOC) and nitrate in groundwater collected at the same time as flux data. Because denitrification is dependent on nitrate $\left(\mathrm{NO}_{3}{ }^{-}\right)$and DOC levels, I predicted higher concentrations of groundwater nitrates and DOC would be related to greater nitrous oxide flux rates from the soil under conditions of high soil moisture and depth to water table.

The fourth objective was to evaluate the influence of soil properties on greenhouse gas flux rates. I predicted $\%$ clay and soil bulk density would be negatively correlated with $\mathrm{CH}_{4}$ uptake by the soil, since $\mathrm{CH}_{4}$ uptake is highly dependent on $\mathrm{CH}_{4}$ diffusion rates into the soil from the atmosphere. I also predicted fluxes of $\mathrm{CO}_{2}$ and $\mathrm{N}_{2} \mathrm{O}$ would follow patterns others have found with regards to bulk density (Xu and Qi 2001), with decreasing flux rates at higher bulk densities due to fewer soil pore spaces for microbial activity.

\section{Methods}

Study Area

The study area consisted of three individual watersheds located within and adjacent to Louisville, Kentucky, USA (Latitude $38^{\circ} 15^{\prime} \mathrm{N}$, Longitude $85^{\circ} 46^{\prime} \mathrm{W}$ ), which has a population of approximately 700,000 with a mean density of 695 people $\mathrm{km}^{-2}$ (U.S. Census Bureau 2008). These were the Beargrass Creek (Middle Fork), Goose Creek, and Harrods Creek (South Fork) watersheds. The watersheds lie adjacent to each other with 
all streams flowing in a primarily westerly direction and eventually emptying into the Ohio River. The three watersheds are of approximately equivalent sizes, containing varying proportions of impervious surface cover.

Beargrass Creek watershed, located in the north-northeast portion of Jefferson County, KY, is approximately $65-\mathrm{km}^{2}$ (Metropolitan Sewer District 1999b) and contains the greatest proportion of impervious surface cover (33\% ISC). This Middle Fork of Beargrass Creek joins the South Fork before flowing into the Ohio River. Several stretches of this stream are featured on Kentucky's Division of Water 303d list, which list streams with impairment issues as well as the pollutant(s) causing the impairment (Kentucky Environmental and Public Protection Cabinet 2008). Since combined sewer overflows (CSOs) commonly occur in this stream, the presence of fecal coliform and sewage are stated as the primary problem pollutants within Beargrass Creek.

Goose Creek is a $50-\mathrm{km}^{2}$ watershed also in Jefferson County, in-between and adjacent to Beargrass and Harrods Creek watersheds (Metropolitan Sewer District 1999a). Of the three watersheds in this study, impervious surface cover is intermediate ( $20 \%$ ISC). The creek is composed of a pair of forks that meet before emptying into the Ohio River. Goose Creek is also listed on the $303 \mathrm{~d}$ list, with cadmium and fecal coliform levels stated as impairment issues.

South Fork Harrods Creek watershed $\left(60 \mathrm{~km}^{2}\right)$, which has the lowest impervious surface cover of the three (10\% ISC), is located primarily in neighboring Oldham County, but extends into northeast Jefferson County. South Fork Harrods Creek eventually joins North Fork Harrods Creek to become Harrods Creek before flowing into the Ohio River. Sections of the larger Harrods Creek network made the $303 \mathrm{~d}$ list in years 
past, specifically the portion passing through Jefferson County which is a more developed area. As in the other two watersheds, fecal coliform has traditionally been a problem (Metropolitan Sewer District 1999c). The current 303d list contains Harrods Creek with fecal coliform as an impairment issue, although this information is for the stream section downstream of where the North Fork and the South Fork Harrods Creek join.

Watershed classification

Watersheds were chosen from digitized catchment layers downloaded from the Louisville/Jefferson Information Consortium (LOJIC) database (LOJIC 2010). LOJIC is a multi-agency effort to maintain a geographic information systems (GIS) database to serve primarily Jefferson County, KY, although limited information is available for a portion of Oldham County, KY. After checking for topographical accuracy, I divided catchments into subcatchments based on stream order and topography from the original LOJIC layers. I further divided subcatchments of higher stream orders with longer reaches into two or three subcatchments. Data layers from the LOJIC database were clipped to each of these subcatchments. Beargrass Creek watershed contained thirteen subcatchments, Goose Creek watershed contained twelve subcatchments, and Harrods Creek watershed contained fourteen subcatchments.

Impervious land use classification was determined using three data layers from the LOJIC database digitized in 2001: buildings, miscellaneous transportation, and roads. The buildings-layer included all built structures, whether commercial or residential. The roads-layer included all paved road surfaces, excluding parking lots. The miscellaneous 
transportation-layer included secondary transportation features such as driveways, parking lots, and sidewalks. The area of land covered by impervious surfaces for each subcatchment was determined using ArcGIS 9 (ESRI, 2010) and was divided by the total land area for each subcatchment to determine the proportion of impervious surfaces covering the landscape.

Categorization of subcatchments into urban, suburban, and rural land use categories was modified from Schuler (1994). For this study, urban subcatchments were defined as containing $\geq 30 \%$ impervious surface cover. Rural subcatchments contained $\leq 10 \%$ impervious surface cover and suburban subcatchments contained between ten and $30 \%$ impervious surface cover. Research sites were classified by land use based on impervious surface cover at three scales. The first and largest areal scale was the subcatchment level, where sites were classified as urban, suburban, or rural based on the subcatchment in which they were located. Impervious surface cover was calculated at two additional and smaller scales surrounding the sites. Site buffers were created using ArcGIS 9 at a radius of $1-\mathrm{km}$ and $500-\mathrm{m}$ from the site center. The proportion of impervious surface cover was determined for these buffers in the same way as for the subcatchments.

Site selection and gas chamber installation

Chosen sites were a subset of those used in previous vegetation studies (see Dissertation Chapters 2 and 3). Thirteen out of 41 sites were chosen based on bank height, land-use classification (insuring similar numbers of urban, suburban, rural catchments), and landowner permission for entry and for groundwater well installation. 
Contingent upon landowner cooperation, I attempted to install a groundwater well at each site. If a well could not be installed due to excessively rocky soils, or if I encountered bedrock before groundwater, then the site was not used, with the following exception. I used one rural site with no water table above bedrock following multiple attempts at other sites and encountering excessively rocky soils before encountering groundwater or bedrock. Groundwater levels were measured in urban, suburban, and rural sites every two weeks from November 2006 to October 2008. Some of the well locations were changed in 2007 due to limited landowner permissions and to capture the variation in bank heights observed (see Dissertation Chapter 2) by land-use type, calculated at the subcatchment scale.

All wells and gas chamber bases were installed by October 2007 , which was a month before any sampling took place. Two circular gas chamber bases $(28.5-\mathrm{cm}$ inside diameter) (Artmor Plastics, Cumberland, MD, USA) were installed per site parallel to the stream one to two meters from the streambank edge, and three meters apart from each other. PVC bases were hammered into the ground as far as possible but no more than six $\mathrm{cm}$ (maximum base height). Periodic measurements of chamber height were taken relative to the ground surface to ensure an accurate air volume determination before measurements were taken. I began sampling with five urban, four suburban, and four rural sites. Four urban and one suburban site were located along Beargrass Creek; one urban, three suburban, and two rural sites along Goose Creek; and one suburban and two rural sites along Harrods Creek. In February 2008, two gas chambers were taken from a suburban site within a state park along Goose Creek; therefore, I abandoned that site. I chose an additional urban site along a first order tributary of Beargrass Creek in July 
2008. The site was located in a remnant woodland area, with the entire riparian zone $(\leq$ 12-m wide throughout) upstream of and at the site being forested.

\section{Groundwater wells}

Nearstream water table depth was determined over a two-year period at these 13 sites by sampling piezometer wells. One piezometer well was installed within 0.5 - to 1meter of the streambank edge in each of six urban, three suburban, and four rural riparian sites. The shafts of the piezometer wells were constructed of poly-vinyl chloride (PVC) pipe with a 1.25 -inch inner diameter to allow for insertion of a measuring tape for determination of groundwater depth from the ground surface. The base of each piezometer was constructed of slotted PVC pipe approximately $300-\mathrm{mm}$ in length. When measurements were begun at the end of 2006, piezometer bases used for measurement were commercially manufactured using three-quarter inch PVC pipe. Throughout 2007 some of those were replaced with self-constructed bases measuring $3.2-\mathrm{cm}$ in diameter to allow enough water to collect inside the well for sampling. Plastic PVC caps were placed at the bottom and top of the well to prevent water and debris from entering the well from above and also to allow water to drain from the well had the water table dropped below the well, as a hole was drilled into the bottom cap. In both the commercial and selfconstructed bases, a mesh filter covered the slots to limit soil and debris entering the well. The piezometer base was attached to the shaft via a PVC collar. PVC cement was used sparingly to attach the collar and bottom cap to minimize potential contamination.

To place the piezometer into the ground I used a $10.2-\mathrm{cm}$ diameter auger to drill a hole large enough in diameter to prevent the well from contacting the sides upon 
placement. I also allowed the top portion of the piezometer to protrude at least $30-\mathrm{cm}$ above-ground to prevent floodwaters from entering the well. Once in place, sand was poured to cover the base of the piezometer to allow for drainage and prevent clogging of the filter surrounding the base. A layer of bentonite clay pellets approximately $15.2-\mathrm{cm}$ deep was poured on top of the sand layer. Water was poured over the pellets to allow for expansion, and native soil was used as the remainder of the fill. Depth to groundwater measurements in the well were made approximately every two weeks from November 2006 to November 2007 using a tape measure coated with Keson ${ }^{\circledR}$ ultra-fine marking chalk. The remainder of the measurements up to October 2008 followed the frequency of soil gas sampling described below.

\section{Soil and site properties}

Soil properties determined at each site visit included soil temperature and gravimetric soil moisture. Soil temperature was determined using a long-stem thermometer, and was measured $50-\mathrm{cm}$ from each chamber during each site visit. Measurements were taken at 5-, 10-, and $15-\mathrm{cm}$ depths to obtain a representative average through the soil column. Air temperature was measured between the two chambers. Gravimetric soil moisture was determined from a 2-cm diameter soil core taken one to 1.2 meters from each chamber, so as not to disturb the soil in and closer to the chamber. Two soil cores for each chamber were obtained to a depth of $15-\mathrm{cm}$ each site visit. Soils were enclosed in plastic bags until later that same day when they were weighed and then dried in an oven at $105^{\circ} \mathrm{C}$ for up to 48 hours. Gravimetric soil moisture content was determined using the following formula: 
Eq. 1

Gravimetric soil moisture $(\%)=100 *$ (Soil wet wt. (g) - Soil dry wt. (g))/Soil dry wt. (g)

Additional soil characteristics were determined from a single soil sample taken inside the chambers in the first half of October 2009 after the last gas samples for the study were collected. Soil cores (10.2-cm diameter and 13-cm long) were taken from the middle of each trace gas chamber using a soil impact corer with polypropylene sleeves. Stones and roots were removed and soils dried at $105^{\circ} \mathrm{C}$ for at least 48 hours. Bulk density was determined for each core using the following formula:

Eq. 2

Bulk density $\left(\mathrm{g} / \mathrm{cm}^{3}\right)=$ Dry soil weight $(\mathrm{g}) /$ Soil core volume $\left(\mathrm{cm}^{3}\right)$

Soil C:N molar ratios for each chamber were determined via combustion in the EAL at the University of Louisville using a Perkin-Elmer Series II 2400 (CHNS/O) analyzer.

Additional soil properties (nutrients and texture) were determined using soil samples pooled from both chambers at each site. Soils were well mixed, and passed through a 2-mm sieve prior to analysis. Soils were analyzed at Brookside Laboratories (308 S. Main Street, New Knoxville, OH, USA; www.blinc.com) for texture (ASTM Standard D422-63 2002) and trace minerals(Bray and Kurtz 1945, Linsay and Norvell 1978, Mehlich 1984), the full list of which can be found in Appendix 13.

Vegetation surrounding each chamber was quantified to use as correlates that could potentially explain some of the variation in gas flux. Percent bare ground and 
herbaceous cover, and shrub and sapling counts were assessed within a 2-m radius of each chamber. Percent cover for herbs and bare ground was assessed using a seven category cover class system (Category $\#=\%$ cover; $1=<1 ; 2=1-5 ; 3=6-25 ; 4=26-50$; $5=51-75 ; 6=76-95 ; 7=>95$ ) with the average midpoints reported. Tree counts were determined within five meter radius of each chamber. Counts are reported as the total within a $12.6-\mathrm{m}^{2}$ area for shrubs and saplings and within a $78.5-\mathrm{m}^{2}$ area for trees. Tree canopy cover within a 5-m radius of the chambers was assessed using a spherical densiometer (Lemmon 1956).

Sampling Well Water for Dissolved Organic Carbon and Nitrate + Nitrite- $N$

DOC samples were collected once per month from November 2007 to October 2008 , except during June 2008 when two collections were made. Samples for determining nitrate+ + nitrite- $\mathrm{N}$ concentration $(\mathrm{mg} / \mathrm{L})$ were also collected once per month from November 2007 to February 2008, and in August and October 2008. However, the collection frequency was doubled from March to July and in September 2008 to follow the gas sampling frequency described below. Prior to well water collection, wells were emptied of water using a peristaltic pump and Nalgene tubing and allowed to fill for at least 30 minutes prior to sampling. This was done to obtain a recent water sample and also to rinse the inside of the tubing. Water samples were collected from wells into 100ml acid-washed Nalgene bottles. Samples were stored on ice until returned to the lab. Samples were then vacuum-filtered through $0.45-\mu \mathrm{m}$ filters (glass fiber, type A/E). Forty $\mathrm{ml}$ aliquots were decanted into sterile glass bottles for determination of dissolved organic carbon (DOC) concentrations. Ten drops of $4 \mathrm{~N} \mathrm{HCl}$ were added to the DOC sample to 
stop microbial activity, and samples were stored at $4^{\circ} \mathrm{C}$. The remainder of the sample was decanted into a sterile $40-\mathrm{ml}$ Falcon tube and either stored at $4{ }^{\circ} \mathrm{C}$ or frozen at $-20^{\circ} \mathrm{C}$ for nitrate+nitrite- $\mathrm{N}$ determination.

DOC concentrations were determined via combustion by the Environmental Analysis Laboratory (EAL) at the University of Louisville using a Shimadzu TOC Model $5050 \mathrm{~A}$ analyzer. Water samples were assessed for nitrate+nitrite-N within seven to ten days following collection. Colorimetric analysis was used following a modified cadmium reduction method from Hach, Inc. (2004a, b). Two different methods were used depending on sample concentration. The low-range method was used for samples containing from 0 - to $0.5-\mathrm{mg} / \mathrm{L}$ nitrate+nitrite-N. The high-range method was used for samples containing from $0.5-$ to $30-\mathrm{mg} / \mathrm{L}$ nitrate + nitrite- $\mathrm{N}$. These methods involve reduction of nitrate to nitrite, followed by conversion to a diazonium salt in the presence of sulfanilic acid. The salt couples with either gentisic acid (high-range) or chromotropic acid (low-range) for color formation. Samples were divided into 5- (for high-range) or 15 -ml (for low-range) aliquots. All samples were warmed to $25^{\circ} \mathrm{C}$ in a water bath prior to analysis. First, for the low-range procedure, the contents of a NitraVer 6 Reagent Powder Pillow were added to the sample, followed by vigorous shaking for 3-min. A 2-min reaction period followed. Ten mls of the sample was decanted into a separate container, followed by addition of a NitraVer 3 Nitrite Reagent Powder Pillow and repeated inversion for $30-\mathrm{sec}$. The sample was allowed to incubate for $15-\mathrm{min}$. One $\mathrm{ml}$ of sample was analyzed in a UV/Vis spectrophotometer with a $1-\mathrm{cm}$ path length at $507-\mathrm{nm}$, with the resulting reading in $\mathrm{mg}$ nitrate + nitrite-N/L. Samples found to contain a greater than 0.5 $\mathrm{mg}$ nitrate + nitrite- $\mathrm{N} / \mathrm{L}$ were subjected to the high-range test. The NitraVer 5 Reagent 
was added to the 5-ml sample, followed by vigorous agitation for 1-min. A 5-min. reaction period followed. One $\mathrm{ml}$ of the sample was read using the same $\mathrm{UV} / \mathrm{Vis}$ spectrophotometer with a $1-\mathrm{cm}$ path length at a wavelength of $500-\mathrm{nm}$.

\section{Measuring net $\mathrm{CO}_{2}$, net $\mathrm{CH}_{4}$ and net $\mathrm{N}_{2} \mathrm{O}$ fluxes from riparian soils}

Soil respiration and $\mathrm{CH}_{4}$ flux was measured from January to October 2008 . Measurements were taken once a month in January, February, August and October. Sampling occurred at two-week intervals from March to July and in September. These trace gas fluxes were measured using closed chambers (purchased from Artmor Plastics Corporation, Cumberland, Maryland, USA) each consisting of a cylindrical lid and base constructed of polyvinyl chloride (PVC). A rubber gasket on the lid formed a seal with the base when in place. The lid had an inner volume of $3.1125-\mathrm{L}$ and the area of the base measured $638-\mathrm{cm}^{2}$. Bases were permanently installed in the ground in October 2007, and volume varied depending on depth of installation, which was measured for each chamber to determine the total air volume of each. During sampling, the chamber lids were placed on the base and fine-needle $50-\mathrm{ml}$ polypropylene syringes were used to collect 20 - to 30 $\mathrm{ml}$ gas samples from sampling ports located in the center of the chamber lids. Collection times occurred at $0,10,20$, and 30 minutes after placement of the chamber lid on the base. Syringes were closed via stopcocks and samples were processed the same or next day. An SRI 8610 gas chromatograph (SRI, Redondo Beach, CA, USA) equipped with a thermal conductivity $\left(\mathrm{CO}_{2}\right)$ and flame ionization $\left(\mathrm{CH}_{4}\right)$ detector and a porapak $\mathrm{Q}$ column was used to process the samples. Gasses used for standardization of chromatogram peaks were $360 \mathrm{ppm} \mathrm{CO} 2$ and $10 \mathrm{ppm} \mathrm{CH}_{4}$. Helium was used as the carrier gas. These trace gas 
fluxes were calculated from the linear rate of change in gas concentration multiplied by the internal volume of the chamber and normalized on a $\mathrm{m}^{2}$ soil surface basis, as follows:

Eq. 3

Flux rate $=\left((\text { Vlid }+ \text { Vbase }) /\left(\mathrm{R}^{*} \mathrm{~T}\right)\right)^{*}(\Delta \text { conc/hour })^{*}($ Cam $) *(24$ hours/day $\left.)\right) /\left(\right.$ Abase- $\left.{ }^{2}\right)$

where Vlid is the volume of the chamber lid $(3.1125 \mathrm{~L})$, Vbase is the volume of the installed chamber base (L), $\mathrm{R}$ is the ideal gas constant $\left(0.0821 \mathrm{~L}^{*} \mathrm{~atm} /(\mathrm{mol} * \mathrm{~K})\right), \mathrm{T}$ is chamber air temperature $(\mathrm{K}), \Delta$ conc is the change in concentration of $\mathrm{CO}_{2}$ or $\mathrm{CH}_{4}$ $(\mathrm{ppm} / 1000 \mathrm{mg} / \mathrm{g} *$ hour $)$, Cam is the atomic mass of carbon $(12-\mathrm{g} / \mathrm{mol})$, and Abase- $\mathrm{m}^{2}$ is the area of the chamber base in square meters $\left(0.0638-\mathrm{m}^{2}\right)$. The unit for flux rate is $\mathrm{mg}$ $\mathrm{CH}_{4}-\mathrm{C}$ or $\mathrm{CO}_{2}-\mathrm{C} / \mathrm{m}^{2} * \mathrm{~d}$

Samples were assessed for $\mathrm{N}_{2} \mathrm{O}$ concentrations at a separate facility (University of New Hampshire Water Resources Research Center, Dr. William McDowell, Jeffrey Merriam), since there was no operational electron capture detector on the SRI 8610 gas chromatograph. Samples were collected once in May, June, July, and August 2008. Samples were collected in the same way as $\mathrm{CO}_{2}$ and $\mathrm{CH}_{4}$, and immediately transferred to $10-\mathrm{ml}$ evacuated glass vials and sealed with rubber septa for storage. Silicon grease was used to reduce leakage from the vials during storage and shipment. Samples were shipped approximately seven to nine days following collection. Five to ten $\mathrm{ml}$ of the air sample was injected into a Hewlett-Packard 5890 Series II (1-ml sample loop) equipped with an electron capture detector. Vials with $\mathrm{N}_{2} \mathrm{O}$ gas controls (500 ppm) were shipped along with the samples to assess the probability of leakage from vials during shipment. The 
decision was made not to include data from the May and June sampling dates since it was determined that leakage occurred in many samples.

\section{Data Analysis}

Principal components analysis (PC-Ord v. 4.41) (McCune and Mefford 1999)and multiple regression analysis (R v. 2.11) (R Development Core Team 2009) were used to assess whether monthly and seasonal differences in $\mathrm{CH}_{4}$ and $\mathrm{CO}_{2}$ fluxes were related to land use and site variables. Principal components analysis was used to assess whether there were patterns in $\mathrm{CH}_{4}$ and $\mathrm{CO}_{2}$ fluxes that could be related to site variables (\%IS, woody species stem counts, $\%$ canopy cover, catchment area, bank height) and soil variables (\% carbon and nitrogen, molar $\mathrm{C}: \mathrm{N}$ ratios, texture, bulk density, $\%$ organic matter). Two sites were removed from these analyses, because one site was determined to be an outlier, as it occurred by itself and caused the other sites to cluster together in a separate corner of the PCA output. The other site was removed because flux data were only collected from July through October for that site. However, these sites were used in subsequent analyses if they did not contribute to heteroscedasticity.

Multiple regression was used to identify distinct soil properties that might affect seasonal differences in site fluxes. Seasons used were winter (January, February, and March), spring (April \& May), summer (June-August), and fall (September \& October). Monthly fluxes were averaged for each site by season and simple regressions were used to choose the strongest two or three main effects to use in each multiple regression model. Land-use differences in depth to groundwater were assessed using repeatedmeasures ANOVA. 


\section{Results}

Site and soil properties

Soil temperature followed air temperature, steadily increasing from freezing and single digit temperatures in January and February and peaking in late August and early September 2008. Increases in soil temperature lagged behind air temperature during the year, with soil temperature rising to between 22 and $23^{\circ} \mathrm{C}$, and air temperature rising to 26 to $28^{\circ} \mathrm{C}$ (Fig. 4-1). No differences in air or soil temperature among land-use categories (urban, suburban, rural - subcatchment scale) were observed. Mean monthly soil moisture was highest in spring and summer and declined from February to October in sites across all land-uses (Table 4-1, Fig. 4-2). Soil moisture was consistently lower throughout the year in urban soils than in either suburban or rural soils (Table 4-1, Fig. 4-2). However, small incremental increases in soil moisture generally followed rain events among all land-use categories. Vegetation coverage and density near the chambers varied between sites and land-use categories (Table 4-2). Urban sites had the highest mean proportion of bare ground within two meters of each chamber. Two suburban sites contained the highest number of trees.

\section{Groundwater}

Both data sets (2006 to 2007, and 2007 to 2008) showed similar patterns regarding bank height and depth to groundwater, with the mean water table depth becoming lower as bank height increased (Fig. 4-3). The slopes of the regression lines for mean water table depth were also similar between years, indicating chronically lower water tables with higher banks. Sites were chosen in part to represent the range of bank 
heights found. As such, only the highest urban bank did not have a similarly paired rural reference (Fig. 4-3b), since none of the rural streambanks I measured in a previous study (see Dissertation Chapter 2) were as high as urban banks. Urban groundwater was also deeper than in either suburban or rural riparian zones due to the higher banks in urban areas (Fig. 4-4). However, no significant differences were detected between urban, suburban, and rural depths to groundwater $(\mathrm{p}=0.44)$. Depths did, however, significantly change over time ( $\mathrm{p}=<0.0001)$, increasing from winter to spring, and decreasing continuously into fall. Groundwater at all sites peaked in March 2008 with a heavy monthly rainfall approaching 46-cm. Depth to groundwater levels decreased gradually from the March peak until October. A small rain pulse in May helped maintain groundwater levels during that month. The scant rainfall that occurred in subsequent months in 2008 was not sufficient to prevent groundwater levels from dropping. Greater recharge of rural groundwater levels occurred during spring rains with average groundwater levels rising 60-cm from November 2007 levels. In contrast, urban groundwater levels rose by $20-\mathrm{cm}$ during the same time period, while suburban groundwater levels were intermediate, rising by approximately $40-\mathrm{cm}$.

\section{Groundwater nitrate+nitrite- $N$ and $D O C$}

Over the 11 month sampling period (Table 4-3), nitrate+nitrite- $\mathrm{N}$ concentrations in groundwater were higher in urban $($ Mean $=2.8-\mathrm{mg} / \mathrm{L} ; \mathrm{SE}=0.8)$ and rural $($ Mean $=$ 2.6-mg/L; $\mathrm{SE}=0.9)$ plots than in suburban plots $($ Mean $=0.09-\mathrm{mg} / \mathrm{L} ; \mathrm{SE}=0.02)$. The highest nitrate+nitrite- $\mathrm{N}$ values in urban areas occurred from January through June 2008, often being at least two orders of magnitude above suburban and rural values during this 
period. In contrast, the highest values in rural areas occurred from May through October, with values equal to those in urban areas from May to July, and exceeding urban values in August through October. Suburban nitrate+nitrite-N values remained relatively low and stable over the year-long period, rarely exceeding $0.1-\mathrm{mg} / \mathrm{L}$. As far as could be determined, rainfall did not vary appreciably within the area by land-use type (Table 4-3), and therefore could not explain the order of magnitude differences in nitrate+nitrite- $\mathrm{N}$ concentration among land uses.

Within a land-use type, however, nitrate + nitrite concentrations followed average rainfall and groundwater levels. Urban mean nitrate + nitrite- $\mathrm{N}$ concentrations and variation were highest during periods of the greatest rainfall and highest groundwater levels in March (Fig. 4-5). Concentrations gradually diminished, falling below 1-mg/L in July. Rural groundwater levels did not start to increase until the relatively wet month of May, and levels peaked following a September pulse of rain. Suburban nitrate+nitrite-N concentrations in groundwater consistently remained below $1-\mathrm{mg} / \mathrm{L}$ for the entire sampling period despite variable precipitation inputs.

Dissolved organic carbon (DOC) showed a more stable trend across land cover categories (Table 4-4), usually ranging between 4- and 7-mg/L. The highest values across land-use categories occurred during winter and summer months. DOC concentrations in urban areas peaked in January and June 2008, with values between 8- and 9-mg/L. Mean suburban values were highest in November 2007 and June 2008, while mean rural values were highest in November 2007 and July 2008. 
Carbon dioxide flux

Carbon dioxide flux varied about 5-fold from January through October 2008 (Fig. 4-6), generally following changes in soil temperature (Fig. 4-1b). PCA indicated that annual mean $\mathrm{CO}_{2}$ flux was related positively with increasing $\%$ impervious surface around the sites from the $0.5 \mathrm{~km}$ to the subcatchment scales (Fig. 4-7). Differences among sites grouped by land use were first distinguishable in June, with mean annual fluxes being consistently highest in urban sites from June through October (Appendix 14). A multiple regression model was used to determine the extent to which impervious surface, calculated at three scales around each site $(0.5-$ and $1-\mathrm{km}$ radii and at the subcatchment scale), and upstream cumulative catchment area could explain the variation in $\mathrm{CO}_{2}$ fluxes among sites. I grouped the data by season due to the effects of seasonal temperatures on gas fluxes. Cumulative catchment area (ha) upstream of the sites ( $p=$ 0.002 ) and $\%$ impervious surface within $1-\mathrm{km}$ of the sites (as opposed to the subcatchment scale; $\mathrm{p}=0.002$ ) explained most of the variation in $\mathrm{CO}_{2}$ flux during summer, with higher \%IS resulting in greater flux rates and increasing catchment area resulting in lower flux rates (two-factor model adjusted $\mathrm{r}^{2}=0.72, \mathrm{p}=0.001$; Table 4-5). One outlier with the highest IS within a $1-\mathrm{km}$ radius $(47 \%)$ and mid-range $\mathrm{CO}_{2}$ flux rate was left out of the analysis to better meet assumptions of homoscedasticity. This site was located in a small city park (Browns Park) along Middle Fork Beargrass Creek, which may have served to reduce the otherwise positive relationships associated with impervious surface on $\mathrm{CO}_{2}$ flux. Fall $\mathrm{CO}_{2}$ flux rates (Table 4-5) were related to \%IS and catchment area in the same way as during summer months, increasing with \%IS ( $p=$ $0.003)$ and decreasing with increasing cumulative catchment area $(\mathrm{p}=0.01)$. 
Multiple regressions revealed relationships between $\mathrm{CO}_{2}$ flux and plot-level environmental variables that varied by season. Winter fluxes (mean flux rates for January, February, and March) positively correlated with \% soil organic matter (\%SOM; $p=0.004$, adj. $r^{2}=0.58$; Fig. 4-8, Table 4-6). One rural site was not used in this analysis due to its negative $\mathrm{CO}_{2}$ flux value, making it an outlier among the sites. It was the only site with no groundwater above bedrock. Spring (April and May mean flux rates) flux differences (natural log transformed) among sites were found to increase with increasing soil bulk density (Fig. 4-9, Table 4-6), indicating a weak trend among spring $\mathrm{CO}_{2}$ fluxes and within the range of bulk density, which was relatively low. More proximate variables related to $\mathrm{CO}_{2}$ flux in the summer were soil temperature $(\mathrm{p}=0.01)$ and soil $\%$ organic matter $(\mathrm{OM})(\mathrm{p}=0.0496)$. Together they explained $43 \%$ of the variation in $\mathrm{CO}_{2}$ flux rates among sites (Table 4-6). Fall $\mathrm{CO}_{2}$ flux rates also increased with soil temperature ( $\mathrm{p}=$ 0.004, adj. $\mathrm{r}^{2}=0.55$; Table 4-6, Fig. 4-10). The same outlier was removed from the regression model for the fall season as for the summer season. No correlations were found between seasonal $\mathrm{CO}_{2}$ flux and either $\mathrm{CH}_{4}$ or $\mathrm{N}_{2} \mathrm{O}$ flux.

\section{Methane flux}

Methane uptake into the soil (methanotrophy) occurred in suburban and urban riparian zones from January to October 2008. Net $\mathrm{CH}_{4}$ emissions from the soil (methanogenesis) primarily occurred in rural riparian zones from January to July 2008. Net methanotrophy occurred in all riparian zones regardless of surrounding land use from July to October 2008 (Fig. 4-11). The range of flux values varied widely among rural sites. Net efflux usually occurred at a single rural site (RG7), with a second rural site 
(RH13) exhibiting net efflux on four of six sampling dates from April through June. These sites were responsible for the large rural peak in March and April. RG7 was typically an outlier because of frequent and sometimes substantial methane release from the soil. Net methane uptake occurred at the other two rural sites on all dates sampled. Net efflux also occurred within some suburban and urban sites, but most sites exhibited net methane uptake on most sampling dates (Appendix 15).

A multiple regression model was used to determine the extent to which impervious surface, calculated at three scales around each site $(0.5-$ and $1-\mathrm{km}$ radii and at the subcatchment scale), and upstream cumulative catchment area could explain the variation in $\mathrm{CH}_{4}$ fluxes among sites. I grouped the data by season due to the effect of temperature on microbial activity and because PCA revealed a methane flux gradient among sites that appeared strongest during fall months (Fig. 4-12 \& 4-13). At the landscape scale, soil uptake of methane increased with increasing subcatchment \%IS (as opposed to within a 1-km radius) during summer months (Table 4-5, Fig. 4-14). Methane uptake rates at sites with less than $15 \%$ IS were between zero and $0.5-\mathrm{mg} \mathrm{CH}_{4}-\mathrm{C} / \mathrm{m}^{2} * \mathrm{~d}$. Both sites that exhibited net methanogensis contained less than $15 \%$ IS in the surrounding subcatchment. Conversely, six of eight sites with greater than $15 \%$ IS had net methane uptake rates greater than $0.5-\mathrm{mg} \mathrm{CH}_{4}-\mathrm{C} / \mathrm{m}^{2} * \mathrm{~d}$. No significant correlations were found between spring and fall methane flux and landscape-scale variables (Table 4$5)$.

Multiple regression analysis revealed relationships between $\mathrm{CH}_{4}$ flux and plotlevel environmental variables. Differences in spring methane fluxes were most strongly correlated with soil temperature, as cooler temperatures resulted in greater methane 
uptake from the atmosphere (Fig. 4-15). Summer flux differences across sites were most strongly correlated with soil \% carbon (Fig. 4-16). Methane uptake decreased linearly as soil $\%$ carbon increased from 2 to $7 \%$. Methane efflux (methanogenesis) became the dominant $\mathrm{CH}_{4}$-related process at the highest soil moisture values (>0.4-g water/g dry mass soil) during the summer months (Fig. 4-17). Fall fluxes were found to significantly correlate with soil C:N ratios and soil moisture (Table 4-6, Fig. 4-18). For the overall multiple regression model, the adjusted coefficient of determination was 0.75 and the pvalue was 0.0004 .

$\mathrm{N}_{2} \mathrm{Oflux}$

Nitrous oxide $\left(\mathrm{N}_{2} \mathrm{O}\right)$ fluxes showed a high degree of variability both among and within sites from August to October 2008, so no land-use level trends could be discerned. August and October mean flux rates $\left(\mathrm{mg} \mathrm{N}_{2} \mathrm{O}-\mathrm{N} / \mathrm{m}^{2} * \mathrm{~d}\right)$ were 0.17 and 0.10 in rural, 0.17 and 0.12 in suburban, and 0.16 and 0.14 in urban riparian zones. Site fluxes decreased dramatically at some sites (RH13, SG13, and UB3) from August/September to October, while fluxes at other sites decreased slightly, remained stable or increased over that interval (Fig. 4-19). Flux rates decreased by at least one-half at four sites and by onequarter to one-third at three other sites from August to October 2008. Rates increased by one-quarter to one-half at four other sites. No significant correlations were detected between $\mathrm{N}_{2} \mathrm{O}$ flux and site variables.

\section{Discussion}

Overall, this project adds to the growing body of literature focused on riparian ecosystem functioning in urban areas. Urban riparian functioning has thus far been 
concentrated on nutrient and pollutant removal (Stander and Ehrenfeld 2010). My study contributes not only to this area, but also to understanding the dynamics of groundwater levels, groundwater DOC and nitrate + nitrite-N, and greenhouse gas in riparian zones in urban and urbanizing catchments.

\section{Groundwater levels}

As expected annual changes in depth to groundwater in these riparian sites reflected rainfall inputs, with rain pulses resulting in higher water tables. Water table levels were highest in rural sites during periods of high rainfall. Urban riparian water levels also rose after rainfall, but not to the same levels as rural water tables. These differences in groundwater recharge between urban, suburban and rural riparian zones are likely a direct consequence of urban features, such as impervious surfaces and drainage structures. These features create a barrier to groundwater flow after precipitation events and allow water to bypass exposed soil by funneling it into drainage features that empty into streams. They also indirectly affect depth to groundwater by causing stream channelization and elevated stream banks, which is part of the urban stream syndrome (Walsh et al. 2005).

Lower water tables were observed among sites with higher streambanks, regardless of land-use in the catchment. However, the highest streambanks and the lowest average water tables occurred in urban areas, which was consistent with my prediction. High banks and lower groundwater recharge rates can create what Groffman et al. (2003) termed "urban hydrological drought," whereby the upper soil layers become dryer, changing soil microbiological processes, including those involved in greenhouse gas flux. 
Water table data coupled with soil moisture data (Table 4-1, Fig. 4-2) indicated that urban hydrological drought does exist in riparian zones within the Louisville Metro area, since urban riparian soils were generally dryer than those in suburban and rural riparian soils.

\section{Groundwater nitrate +nitrite- $N$ and $D O C$}

Nitrate+nitrite- $\mathrm{N}$ occurred in greater concentrations in urban and rural plots, peaking at different times of the year. The highest nitrate+nitrite- $\mathrm{N}$ concentrations $(>0.5-$ $\mathrm{mg} / \mathrm{L}$ ) generally occurred at a single urban site (UB3) and a single rural site (RG7). Both sites were located adjacent to housing developments with a house within $50 \mathrm{~m}$ of the sites, and both were at the end of a cul-de-sac. Since nitrate levels peaked coinciding with rainfall events, runoff from fertilized lawns could have contributed to the high levels of nitrate+nitrite-N within the groundwater at these two sites.

Other studies have found that riparian vegetation dynamics influence soil nitrogen dynamics and groundwater concentrations of nitrate. Nearstream groundwater $\mathrm{NO}_{3}{ }^{-}$ concentrations were found to increase four-fold with uprooted canopy tree disturbance in a mountainous region of North Carolina, although elimination of shrubs at other sites had no significant effect (Yeakley et al. 2003). This suggested that canopy trees, especially at the root-soil interface, were primarily responsible for control of nitrates in groundwater. Exotic species invasions have variable effects on soil nitrate concentrations, with effects primarily expressed on $\mathrm{N}$ mineralization and nitrification rates, both increasing with plant species invasions (Ehrenfeld 2003). My suburban sites had the greatest number of trees

$\left(>1-\mathrm{m}\right.$ high, $\geq 2.54-\mathrm{cm}$ diameter at breast height) within a $78.5-\mathrm{m}^{2}$ area surrounding the groundwater wells and the lowest groundwater nitrate concentrations. The absence of 
trees at urban and rural sites could have contributed to the relatively higher nitrate+nitrite- $\mathrm{N}$ concentrations. In addition, the two urban sites with the highest groundwater nitrate+nitrite-N concentrations $(>1-\mathrm{mg} / \mathrm{L})$ from January to June 2008 (UB3 and UB5) also had the greatest densities of the exotic shrub L. maackii (see Appendix 9 and Dissertation Chapter 2 for sampling methods). However, the rural site (RG7) with the highest groundwater nitrate+nitrite-N concentrations (> 1-mg/L) from May to October 2008 did not contain any exotic shrubs (see Appendix 9 and Dissertation Chapter 2 for sampling methods). These conflicting findings make drawing inferences regarding increased groundwater nitrate+nitrite- $\mathrm{N}$ concentrations and exotic shrubs invasions more complicated.

The only discernable pattern regarding nearstream groundwater DOC concentrations were related to seasonal peaks. Table $4-4$ shows winter and summer peaks among all land-use types in 2008. Average rural DOC seasonal peaks were the highest overall, reaching $9.89-\mathrm{mg} / \mathrm{L}$ in November and $10.51-\mathrm{mg} / \mathrm{L}$ in July. Suburban areas had the lowest average seasonal peaks, with concentrations between 7 - and $7.5-\mathrm{mg} / \mathrm{L}$. Urban areas had seasonal peaks between 8 - and 9-mg/L. Summer DOC peaks were observed in a coastal ecosystem in Sweden, with the increases attributed to low phosphate concentrations inhibiting breakdown of DOC by limiting microbial activity (Zweifel et al. 1995). Other possible explanations for the summer and winter peaks include root exudates and leachable organic matter from the litter layer (Wright and Coleman 2002, Yeakley et al. 2003), which have both been found to increase soil carbon concentrations. 
$\mathrm{CO}_{2}$ flux

At the landscape scale, $\%$ impervious surface within a $1-\mathrm{km}$ site radius was positively correlated with $\mathrm{CO}_{2}$ flux rates during both summer and fall (Table 4-5), supporting my prediction. Cumulative catchment area, another landscape scale variable, was negatively correlated with $\mathrm{CO}_{2}$ flux rates (Table 4-5), with decreasing flux rates occurring with increasing catchment area (ha). Explanations for this trend are less easily made. However, Jones and Mulholland (1998) found lower in-stream partial pressures of $\mathrm{CO}_{2}$ with downstream distance in a stream system in the Great Smoky Mountains National Park, which they attributed to the declining influence of groundwater on stream chemistry from upstream to downstream. Similar mechanisms may be responsible for this decline in $\mathrm{CO}_{2}$ in both situations, and further study may be warranted if similar findings can be replicated in other systems.

Carbon dioxide flux demonstrated seasonal variations related to plot-level environmental variation as well. As expected, winter $\mathrm{CO}_{2}$ flux was the lowest because of cold temperatures, while $\mathrm{CO}_{2}$ flux and soil temps peaked in mid-summer (Fig. 4-1b \& 46). Many of the soils were frozen early in the season (January), and began to thaw later in the season (March). Summer and fall $\mathrm{CO}_{2}$ flux rate differences among sites were positively correlated with variation among sites in soil temperature (Table 4-6, Fig. 4-10). This correlation between soil $\mathrm{CO}_{2}$ fluxes with temperature is expected and consistent with many other findings (Edwards 1975, Crill 1991, Davidson et al. 1998, Rustad et al. 2000, Xu and Qi 2001). Percent soil organic matter (SOM) was positively correlated with winter and summer $\mathrm{CO}_{2}$ flux rates (Table 4-6, Fig. 4-8), which was also found by $\mathrm{Xu}$ and Qi (2001) during summer months under a ponderosa pine forest. Spring $\mathrm{CO}_{2}$ flux rates 
were positively, though not significantly, correlated with increasing soil bulk density (Fig. 4-9). This was surprising, and opposite of my prediction, as a higher soil density would indicate a more compact soil with smaller pore spaces for gaseous diffusion. However, these soils were not highly compacted, relatively speaking, with the highest bulk density being $0.73-\mathrm{g}$ soil $/ \mathrm{cm}^{3}$. In the same study referenced above, Xu and Qi (2001) found a negative correlation between $\mathrm{CO}_{2}$ efflux and bulk density, attributing the finding to limited pore space for microbial activity. Therefore, the range of bulk densities at my research sites may be too low to detect a similar pattern.

$\mathrm{CH}_{4} \mathrm{flux}$

Only one of my predictions regarding $\mathrm{CH}_{4}$ was supported by the data within this study. I found a weak relationship between $\mathrm{CH}_{4}$ flux and land-use context (Table 4-5, Fig. 4-14), where the greatest methane uptake rates occurred in more urban sites (determined at the subcatchment scale), whose soils were typically driest in the summer and fall. Groffman et al. (2006) found $\mathrm{CH}_{4}$ uptake rates correlated with land-use context in Baltimore forests. Land-use context was related to high-fertility and low-fertility sites, with high fertility sites occurring primarily in urban environments and low-fertility sites in rural environments. Rates of methane uptake were lower in high fertility forested urban sites than in low fertility rural sites. I found the opposite trend at my sites, with increasing rates of $\mathrm{CH}_{4}$ uptake with increasing $\% \mathrm{IS}$, which may have been due to more proximal scale environmental variation.

Higher spring temperatures resulted in lower rates of $\mathrm{CH}_{4}$ uptake within these riparian sites. Castro (1995) revealed methane uptake rates are dependent on temperature 
within the range of 0 and $10^{\circ} \mathrm{C}$, increasing as temperature increases. Above $10^{\circ} \mathrm{C}$ methane uptake becomes independent of temperature in temperate forest soils. Incubation of forest soil cores has also revealed no relationship between soil temperature above $10^{\circ} \mathrm{C}$ and methane efflux (Crill 1991). This was attributed to a shift from biological to diffusional controls over methane uptake. During spring thaw, uptake rates responded to temperature since substrate $\left(\mathrm{CH}_{4}\right.$ for methanotrophs) was plentiful and microbes increased their activity in response to the temperature increase. Into summer, substrate became limiting as microbial activity increased in response to the soil temperature, and diffusion of $\mathrm{CH}_{4}$ into the soil became the limiting factor controlling rates of $\mathrm{CH}_{4}$ uptake. The sites with higher spring temperatures and lower net $\mathrm{CH}_{4}$ uptake rates suggest substrate-limitation of methanotrophy. Average spring temperatures were above $10^{\circ} \mathrm{C}$ at my sites, which potentially explains the weak trend between temperature and $\mathrm{CH}_{4}$ uptake rates.

Patterns in $\mathrm{CH}_{4}$ flux rates during summer and fall were related to soil moisture and soil carbon and nitrogen concentrations. Soil moisture acted as a switch by determining whether the soil was a net consumer or emitter of $\mathrm{CH}_{4}$ (Fig. 4-17 \& 4-18b, Table 4-6), as methanogenesis is an anaerobic process and soil methane uptake is an aerobic process. Ambus and Christensen (1995) found similar results regarding $\mathrm{CH}_{4}$ flux and soil moisture in a riparian site where static chambers, arranged along a topogradient from upslope to downslope, were used to record methane efflux in the flood plain. They found a significant increase in methane efflux from soils with higher $\%$ water-filled pore space (WFPS) and higher \% soil organic matter (SOM). Castro et al. (1995) found experimental evidence for negative control of methane consumption by soils at levels of 
soil moisture (measured as \% WFPS) between 60 and 100\% in Harvard Forest in Massachusetts. Whalen et al. (1990) found the lowest methane consumption rates occurred above $40 \%$ and below $5 \%$ soil moisture by weight. Reported optimum values for gravimetric soil moisture (g soil water/g dry soil) in relation to methane uptake occur between the ranges of 10-30\% (Whalen et al. 1990, Torn and Harte 1996). In my riparian systems soil moisture values above $\sim 0.35$-g water/g dry soil, slightly higher moisture values than these other studies, promoted net methane efflux from the soil (Fig. 4-17). One site in particular (RG7) exhibited $\mathrm{CH}_{4}$ efflux at nearly every date measured. This site also had some of the highest monthly soil moisture values (Table 4-1) and an average depth to groundwater of $91-\mathrm{cm}$, and the highest groundwater nitrate concentrations from May to October 2008. Net methane uptake occurred at lower soil moisture levels; however, these soil moisture values did not explain variation in my data set.

In summer, net $\mathrm{CH}_{4}$ uptake increased with decreasing soil \% carbon (Fig. 4-16, Table 4-6). This could partially explain increasing methane uptake rates with increasing subcatchment \%IS mentioned previously, since soil \% carbon was negatively correlated with \%IS (Pearson's $r=-0.69$ ). However, soil \% carbon was determined from fall soil collects and they may have been slightly different had they been taken during summer months. In fall, net $\mathrm{CH}_{4}$ uptake increased with decreasing soil C:N ratios (Fig. 4-18a, Table 4-6). The relationship with $\mathrm{C}: \mathrm{N}$ ratios was consistent with responses others have found regarding flux rates and soil nitrogen concentrations. Steudler et al. (1989) found $\mathrm{CH}_{4}$ uptake increased with concentrations of soil nitrogen, particularly ammonium, via fertilization in southeastern temperate forests. This was believed to occur due to increased nitrifier activity, particularly Nitrosomonas europaea, which also possess the 
ability to oxidize $\mathrm{CH}_{4}$ (Jones and Morita 1983). Since higher soil nitrogen concentrations would lead to lower $\mathrm{C}: \mathrm{N}$ ratios (high fertility), and greater methane uptake, nitrifier activity may have been responsible for this pattern within these sites. From a soil fertility perspective, my results are opposite those found by Groffman et al. (2006), who found lower rates of $\mathrm{CH}_{4}$ uptake at high fertility (low $\mathrm{C}: \mathrm{N}$ ) urban sites. However, in my study, no link between land-use and fall $\mathrm{CH}_{4}$ flux rates was found (Table 4-5).

No patterns were found regarding winter $\mathrm{CH}_{4}$ flux between sites or with site or soil variables, which may have been due to the frozen soils. Gulledge and Schimel (2000) found no relationship between climatic factors and methane consumption within taiga forests in Alaska, likely due to the semi-arid nature of the area's climate. In conclusion, net methane flux rates within my riparian sites appear to be controlled by seasonal and local site variables rather than factors operating at the land-use scale.

$\mathrm{N}_{2} \mathrm{Oflux}$

Nitrous oxide flux rate differences, while not correlating with any environmental variables, did show a dramatic seasonal decrease at many sites from August to October. Average soil temperature during this period decreased from $23^{\circ} \mathrm{C}$ to approximately $16^{\circ} \mathrm{C}$. This likely contributed to the decrease in $\mathrm{N}_{2} \mathrm{O}$ flux at some of the sites, although it does not explain steady or increased flux at other sites. As for $\mathrm{CH}_{4}$ flux, interpreting the dynamics of nitrous oxide fluxes is complicated by the fact that it is formed by both aerobic (nitrification) and anaerobic (denitrification) microbial processes. Davidson et al. (1993) found nitrification to be the dominant source of $\mathrm{N}_{2} \mathrm{O}$ production in a tropical forest soil following soil wetting after the dry season. This could not explain variation in 
my data however, as no large rain pulses occurred prior to my sampling dates, and no consistent responses were observed among my sites either by land use or proximity to one another. Others have found $\mathrm{N}_{2} \mathrm{O}$ emissions varied with soil $\mathrm{NO}_{3}{ }^{-}$concentrations, soil organic matter (SOM), and $\mathrm{pH}$ among a range of habitat types (Ambus and Christensen 1995). Arable and coastal grasslands exhibited the highest annual mean $\mathrm{N}_{2} \mathrm{O}$ emissions from among seven different habitat types, including forested and riparian sites. While

none of my predictions were supported, further research should also include measures of in situ nitrification rates and soil moisture monitoring to determine potential associations with $\mathrm{N}_{2} \mathrm{O}$ flux in riparian sites, and modeling a mechanistic partitioning between denitrification and nitrification as explanations for seasonal and land-use scale variation in emissions of this important greenhouse gas.

\section{Conclusions}

Depth to water table was lower in urban areas, as expected, since urban areas had the highest stream banks (Fig. 4-3 \& 4-4). In addition, the degree of groundwater recharge in urban areas during spring rains was much less than in rural areas, demonstrating the effect of impervious surface runoff and structural bypasses on riparian groundwater levels. The absence of recharge means the upper soil layers are never as saturated as in rural areas and soil moisture remained lower in the urban riparian areas as well. Therefore I found evidence to support the notion that urban hydrological drought exists in riparian areas in urban and urbanizing catchments in Louisville.

Carbon dioxide flux rates increased with increasing soil temperatures among all sites (Fig. 4-1 \& 4-6). Site flux difference and potential explanatory variables, however, 
changed in response to season, with winter fluxes influenced by \% SOM (Fig. 4-8). As temperatures increased in the spring, the degree of $\mathrm{CO}_{2}$ release was better explained by soil bulk density (Fig. 4-9). However, the trend was opposite of what I expected, with greater flux rates occurring in the denser soils. However, soil bulk density in these systems ranged only from $0.49-$ to $0.73-\mathrm{g} / \mathrm{cm}^{3}$, and were not considered to be compacted. Soil temperatures played the greatest role in explaining land-use patterns of $\mathrm{CO}_{2}$ release during the hottest months and into fall, with urban riparian sites releasing more $\mathrm{CO}_{2}$ than suburban and rural sites.

Explaining patterns of net soil $\mathrm{CH}_{4}$ flux rates is more complex since net flux is controlled by both anaerobic and aerobic processes. Soil temperature weakly correlated with site flux rates during the spring thaw, with lower net $\mathrm{CH}_{4}$ uptake rates at higher spring temperatures attributed to substrate limitation (atmospheric $\mathrm{CH}_{4}$ diffusion into the soil) of microbial activity (Fig. 4-14). As soil temperatures and soil microbial activity increased, other site differences began to affect patterns of $\mathrm{CH}_{4}$ uptake and efflux. Summer net $\mathrm{CH}_{4}$ uptake rates were negatively correlated with soil \% carbon (Fig. 4-16). Differences in fall $\mathrm{CH}_{4}$ uptake rates were negatively correlated with soil C:N ratios (Fig. 4-18a). Soils with C:N values below 23 are associated with higher net annual nitrification potential. The latter half of the summer of 2008 was one of prolonged drought. Precipitation events in the fall could have stimulated the activity of nitrifying bacteria, which are also known to take up $\mathrm{CH}_{4}$ (Jones and Morita 1983, Bedard and Knowles 1989). This offers one potential explanation for the negative correlation with soils C:N ratios. Potential effects of high soil moisture on the net balance between methanogenic and methanotrophic activity were evidenced during summer and fall seasons. Higher soil 
moisture values (greater than $\sim 0.35$-g soil water/g dry soil) resulted in a shift from net methanotrophy to net methanogenesis. Patterns of net methane uptake, however, were not explained by variation in soil moisture below $\sim 0.35-\mathrm{g}$ soil water/g dry soil.

$\mathrm{N}_{2} \mathrm{O}$ efflux rates showed a strong seasonal response between August and October, decreasing from hot and dry August to cooler October. No measured environmental site differences could explain the flux differences across sites. Groffman et al. (2006) found natural soil factors had greater control over soil nitrogen cycling dynamics, with higher $\mathrm{N}_{2} \mathrm{O}$ flux rates attributed to finer textured soils. The differences could also be a function of the dual processes in which $\mathrm{N}_{2} \mathrm{O}$ is released from the soil, one process being aerobic (nitrification) and one being anaerobic (denitrification). 
Table 4-1: Gravimetric soil water content (g water/g oven dry soil) in the upper $15-\mathrm{cm}$ horizons within two meters of streambanks in urban, suburban, and rural riparian sites. Urban subcatchments contain $\geq 30 \%$ impervious surface cover, rural subcatchments contain $\leq 10 \%$ impervious surface cover, and suburban subcatchments contain values in between 10 and $30 \%$ impervious surface cover. Site names beginning with $\mathrm{R}, \mathrm{S}$, and $\mathrm{U}$ are located in rural, suburban, and urban subcatchments, respectively. The second letter in the site name indicates whether the site is located in Harrods $(H)$, Goose $(G)$, or Beargrass (B) creek watershed. N/A indicates the soils were frozen.

\begin{tabular}{l|cccccccccc} 
Site & Jan. & Feb. & March & April & May & June & July & Aug. & Sept. & Oct. \\
\hline RG4 & N/A & 0.44 & 0.36 & 0.34 & 0.32 & 0.32 & 0.31 & 0.22 & 0.21 & 0.21 \\
RG7 & N/A & 0.41 & 0.56 & 0.53 & 0.52 & 0.56 & 0.54 & 0.47 & 0.48 & 0.45 \\
RH13 & 0.04 & 0.39 & 0.38 & 0.38 & 0.34 & 0.33 & 0.23 & 0.12 & 0.12 & 0.12 \\
RH5 & 0.05 & N/A & 0.37 & 0.37 & 0.35 & 0.38 & 0.32 & 0.17 & 0.21 & 0.18 \\
Mean: & $\mathbf{0 . 0 2}$ & $\mathbf{0 . 3 1}$ & $\mathbf{0 . 4 2}$ & $\mathbf{0 . 4 0}$ & $\mathbf{0 . 3 8}$ & $\mathbf{0 . 4 0}$ & $\mathbf{0 . 3 5}$ & $\mathbf{0 . 2 5}$ & $\mathbf{0 . 2 6}$ & $\mathbf{0 . 2 4}$ \\
& & & & & & & & & & \\
SB12 & N/A & 0.37 & 0.42 & 0.36 & 0.35 & 0.33 & 0.25 & 0.15 & 0.17 & 0.18 \\
SG13 & N/A & 0.61 & 0.46 & 0.45 & 0.42 & 0.42 & 0.42 & 0.36 & 0.37 & 0.30 \\
SG9 & N/A & 0.42 & 0.41 & 0.38 & 0.35 & 0.33 & 0.28 & 0.20 & 0.21 & 0.21 \\
Mean: & N/A & $\mathbf{0 . 4 7}$ & $\mathbf{0 . 4 3}$ & $\mathbf{0 . 4 0}$ & $\mathbf{0 . 3 8}$ & $\mathbf{0 . 3 6}$ & $\mathbf{0 . 3 2}$ & $\mathbf{0 . 2 4}$ & $\mathbf{0 . 2 5}$ & $\mathbf{0 . 2 3}$ \\
& & & & & & & & & & \\
UB1 & N/A & N/A & 0.33 & 0.30 & 0.29 & 0.27 & 0.25 & 0.16 & 0.17 & 0.17 \\
UB14 & N/A & N/A & NA & NA & NA & NA & 0.40 & 0.26 & 0.23 & 0.26 \\
UB2 & 0.36 & 0.38 & 0.33 & 0.32 & 0.31 & 0.27 & 0.22 & 0.16 & 0.17 & 0.15 \\
UB3 & 0.36 & 0.39 & 0.37 & 0.36 & 0.35 & 0.33 & 0.30 & 0.20 & 0.18 & 0.15 \\
UB5 & 0.31 & N/A & 0.36 & 0.31 & 0.30 & 0.21 & 0.23 & 0.14 & 0.13 & 0.11 \\
UG8 & 0.00 & 0.33 & 0.35 & 0.30 & 0.33 & 0.29 & 0.26 & 0.18 & 0.15 & 0.17 \\
Mean: & $\mathbf{0 . 2 1}$ & $\mathbf{0 . 2 2}$ & $\mathbf{0 . 3 5}$ & $\mathbf{0 . 3 2}$ & $\mathbf{0 . 3 2}$ & $\mathbf{0 . 2 8}$ & $\mathbf{0 . 2 8}$ & $\mathbf{0 . 1 8}$ & $\mathbf{0 . 1 7}$ & $\mathbf{0 . 1 7}$
\end{tabular}


Table 4-2: Site mean vegetation data surrounding the static chambers at riparian sites. Percent bare ground and herbaceous cover, as well as shrub and sapling counts were assessed within a $12.6 \mathrm{~m}^{2}$ area (2-m radius) surrounding each chamber. Tree counts were assessed within a $78.5 \mathrm{~m}^{2}$ area (5-m radius) surrounding each chamber. Canopy cover was determined directly over each chamber. Urban subcatchments contain $\geq 30 \%$ impervious surface cover, rural subcatchments contain $\leq 10 \%$ impervious surface cover, and suburban subcatchments contain values in between 10 and $30 \%$ impervious surface cover. Site names beginning with $\mathrm{R}, \mathrm{S}$, and $\mathrm{U}$ are located in rural, suburban, and urban subcatchments, respectively. The second letter in the site name indicates whether the site is located in Harrods $(\mathrm{H})$, Goose $(\mathrm{G})$, or Beargrass (B) creek watershed.

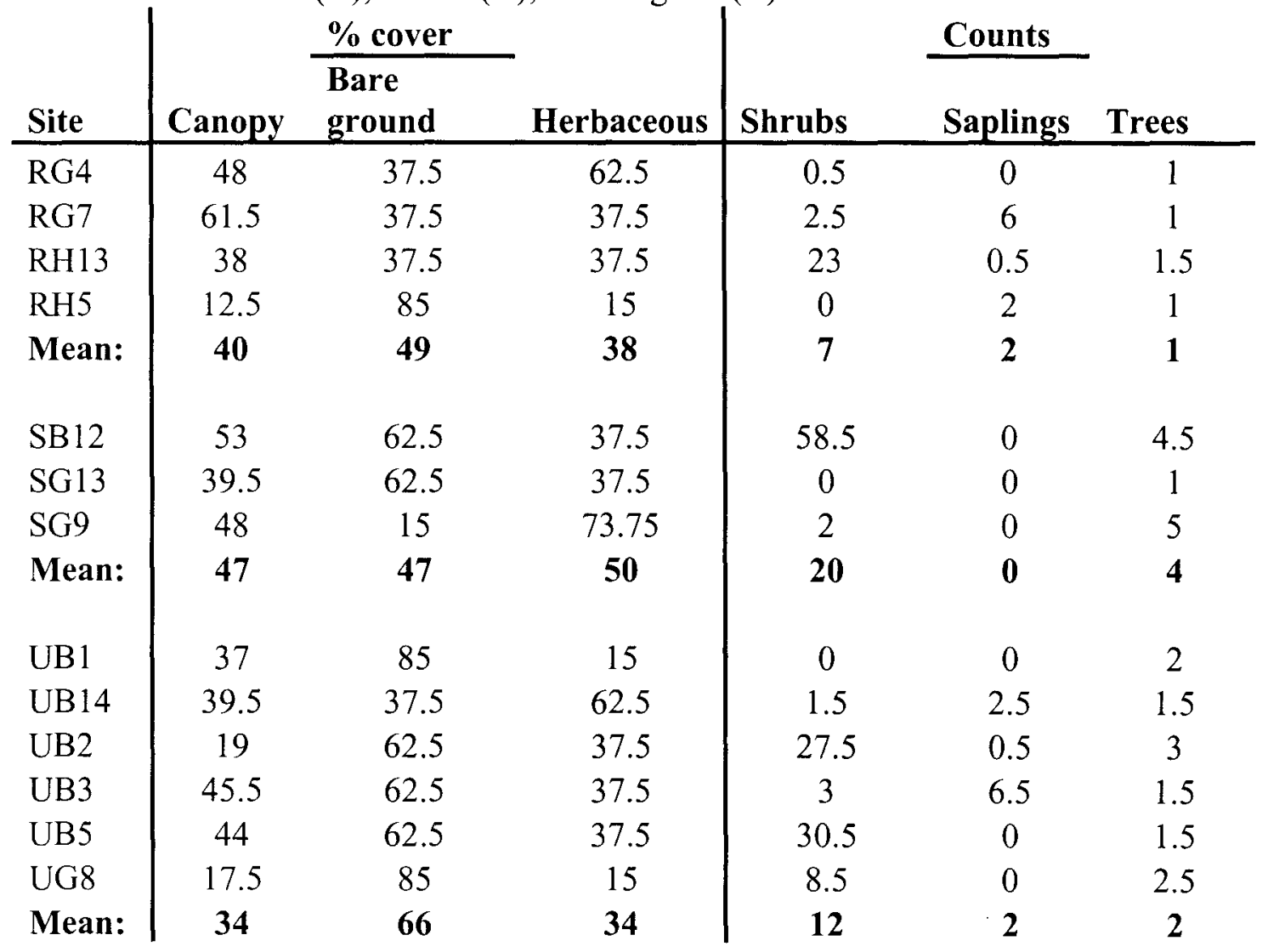


Table 4-3: Riparian groundwater nitrate + nitrite- $N$ monthly means $( \pm$ S.E. $)$ for subcatchment land-use categories and total monthly rainfall from November 2007 to October 2008. Nitrate+nitrite- $\mathrm{N}$ values are means from five or six urban, 3 suburban, and 3 rural sites. Rainfall was determined from a single location monitored by the Louisville Metropolitan Sewer District within Goose (suburban/rural) and Beargrass Creek (Urban) watersheds. Average rainfall is the mean of both stations. Urban subcatchments contain $\geq$ $30 \%$ impervious surface cover, rural subcatchments contain $\leq 10 \%$ impervious surface cover, and suburban subcatchments contain values in between 10 and $30 \%$ impervious surface cover.

\begin{tabular}{|c|c|c|c|c|c|c|}
\hline \multirow[b]{2}{*}{ Month } & \multicolumn{2}{|c|}{$\begin{array}{l}\text { Nitrate+nitrite-N - mg/L } \\
\text { (SE) }\end{array}$} & \multirow[b]{2}{*}{ Rural } & \multirow{2}{*}{$\begin{array}{l}\text { Rainfall } \\
(\mathrm{cm})\end{array}$} & \multirow[b]{2}{*}{$\begin{array}{l}\text { Suburban/ } \\
\text { Rural } \\
\end{array}$} & \multirow[b]{2}{*}{$\begin{array}{l}\text { Avg. } \\
\text { Rain }\end{array}$} \\
\hline & Urban & Suburban & & & & \\
\hline Nov. & $0.80(.29)$ & $0.33(0.16)$ & $0.35(0.07)$ & 7.1 & 8.3 & 7.7 \\
\hline Jan. & $13.17(8.51)$ & $0.082(0.01)$ & $0.23(0.09)$ & 8.3 & 8.2 & 8.25 \\
\hline March & $13.84(8.34)$ & $0.12(0.01)$ & $0.14(0.06)$ & 26.7 & 27.1 & 26.9 \\
\hline April & $6.60(6.53)$ & $0.10(0.02)$ & $0.087(0.02)$ & 15.7 & 13 & 14.4 \\
\hline May & $6.66(4.83)$ & $0.049(0.01)$ & $7.29(4.71)$ & 15.2 & 15.6 & 15.4 \\
\hline June & $14.50(8.09)$ & $0.068(0.02)$ & $12.76(9.35)$ & 7.6 & 10.3 & 8.9 \\
\hline July & $0.10(0.01)$ & $0.068(0.01)$ & $0.16(0.07)$ & 14.6 & 9.3 & 12 \\
\hline August & $0.10(0.02)$ & $0.12(0.05)$ & $4.42(\mathrm{~N} / \mathrm{A})$ & 1.4 & 1.2 & 1.3 \\
\hline Sept. & $0.13(0.04)$ & $0.057(0.01)$ & $13.57(4.10)$ & 3.4 & 5.2 & 4.3 \\
\hline Oct. & $0.15(0.06)$ & $0.046(0.003)$ & $8.20(8.09)$ & 2.1 & 2.2 & 2.1 \\
\hline
\end{tabular}


Table 4-4: Riparian groundwater dissolved organic carbon (DOC) monthly means ( \pm S.E.) for subcatchment land-use categories and total monthly rainfall. DOC values are means from five or six urban, three suburban, and three rural sites. Rainfall was determined from a single location monitored by the Louisville Metropolitan Sewer District within Goose (suburban/rural) and Beargrass Creek (Urban) watersheds. Average rainfall is the mean of both stations. Urban subcatchments contain $\geq 30 \%$ impervious surface cover, rural subcatchments contain $\leq 10 \%$ impervious surface cover, and suburban subcatchments contain values in between 10 and $30 \%$ impervious surface cover.

\begin{tabular}{|c|c|c|c|c|c|c|}
\hline \multirow[b]{2}{*}{ Month } & \multicolumn{2}{|c|}{ DOC - mg/L (SE) } & \multirow[b]{2}{*}{ Rural } & \multirow{2}{*}{$\begin{array}{l}\text { Rainfall } \\
(\mathrm{cm})\end{array}$} & \multirow[b]{2}{*}{$\begin{array}{l}\text { Sub/ } \\
\text { Rural }\end{array}$} & \multirow[b]{2}{*}{$\begin{array}{l}\text { Avg. } \\
\text { Rain }\end{array}$} \\
\hline & Urban & Suburban & & & & \\
\hline November & $6.23(1.07)$ & $7.31(1.53)$ & $9.89(1.39)$ & 7.1 & 8.3 & 7.7 \\
\hline January & $8.06(1.94)$ & $5.48(0.48)$ & $4.58(0.21)$ & 8.3 & 8.2 & 8.25 \\
\hline March & $4.17(0.60)$ & $4.58(1.18)$ & $4.69(0.85)$ & 26.7 & 27.1 & 26.9 \\
\hline April & $5.75(0.44)$ & $6.74(0.53)$ & $6.08(1.40)$ & 15.7 & 13 & 14.4 \\
\hline May & $4.28(1.10)$ & $5.27(0.81)$ & $6.29(1.38)$ & 15.2 & 15.6 & 15.4 \\
\hline June & $8.76(0.88)$ & $7.43(0.27)$ & $6.59(0.83)$ & 7.6 & 10.3 & 8.9 \\
\hline July & $6.60(1.12)$ & $5.47(0.66)$ & $10.51(6.33)$ & 14.6 & 9.3 & 12 \\
\hline August & $5.81(0.57)$ & $5.54(0.30)$ & $5.62(\mathrm{NA})$ & 1.4 & 1.2 & 1.3 \\
\hline September & N/A & N/A & $\mathrm{N} / \mathrm{A}$ & 3.4 & 5.2 & 4.3 \\
\hline October & $5.85(0.66)$ & $5.85(0.12)$ & $6.92(0.87)$ & 2.1 & 2.2 & 2.1 \\
\hline
\end{tabular}


Table 4-5: Relationships between landscape-level soil factors and $\mathrm{CO}_{2}$ and $\mathrm{CH}_{4}$ fluxes in riparian areas across an urban-to-rural land-use gradient.

\begin{tabular}{|c|c|c|c|c|c|c|}
\hline $\begin{array}{c}\text { Greenhouse } \\
\text { Gas }\end{array}$ & Season & Variables & $\begin{array}{l}\text { Ind } p- \\
\text { value }\end{array}$ & $\begin{array}{c}\text { Model } \\
\text { p- } \\
\text { value }\end{array}$ & $\begin{array}{c}\text { Adj. } \\
r^{2}\end{array}$ & Model Equation \\
\hline \multirow{3}{*}{$\begin{array}{c}\text { Carbon } \\
\text { dioxide (mg } \\
\mathrm{CO}_{2^{-}} \\
\mathrm{C} / \mathrm{m}^{2} / \text { day) }\end{array}$} & Spring & $\begin{array}{c}\% \mid \mathrm{S}-1 \\
\mathrm{~km} \\
\text { Catch. } \\
\text { area }\end{array}$ & $\begin{array}{l}0.39 \\
0.33 \\
\end{array}$ & 0.46 & -0.03 & \\
\hline & Summer & $\begin{array}{l}\% \mid \mathrm{IS}-1 \\
\mathrm{~km} \\
\text { Catch. } \\
\text { area }\end{array}$ & $\begin{array}{l}0.002 \\
0.002 \\
\end{array}$ & 0.001 & 0.72 & $\begin{array}{c}y=43(\% \mid S)-32 \text { (Catch. } \\
\text { area) }+2449\end{array}$ \\
\hline & Fall & $\begin{array}{l}\% \mathrm{IS}-1 \\
\mathrm{~km} \\
\text { Catch. } \\
\text { area }\end{array}$ & $\begin{array}{l}0.003 \\
0.01 \\
\end{array}$ & 0.002 & 0.65 & $\begin{array}{c}y=43(\% \mid S)-26 \text { (Catch } \\
\text { area })+1618\end{array}$ \\
\hline \multirow{3}{*}{$\begin{array}{c}\text { Methane (mg } \\
\mathrm{CH}_{4^{-}} \\
\mathrm{C} / \mathrm{m}^{2} / \text { day) }\end{array}$} & Spring & $\begin{array}{c}\text { \%IS - SC } \\
\text { Catch. } \\
\text { area }\end{array}$ & $\begin{array}{l}0.31 \\
0.24 \\
\end{array}$ & 0.37 & 0.02 & \\
\hline & Summer & $\% \mathrm{IS}-\mathrm{SC}$ & - & 0.06 & 0.21 & $y=-0.02(\%$ IS) -0.03 \\
\hline & Fall & $\begin{array}{c}\% \text { IS - SC } \\
\text { Catch. } \\
\text { area }\end{array}$ & $\begin{array}{l}0.26 \\
0.34\end{array}$ & 0.3 & 0.06 & \\
\hline
\end{tabular}

$\% \mathrm{IS}-1 \mathrm{~km}=\%$ impervious surface within $1 \mathrm{~km}$ of the research sites

$\% \mathrm{IS}-\mathrm{SC}=\%$ impervious surface within the subcatchment in which the site is located.

Catch. area = cumulative catchment area (ha) within and upstream of the subcatchment in which the site is located.

Ind. $p$-value $=p$-values for individual explanatory variables in a multiple regression model. 
Table 4-6: Relationships between plot-level soil factors and $\mathrm{CO}_{2}$ and $\mathrm{CH}_{4}$ fluxes in riparian areas across an urban-to-rural land-use gradient.

\begin{tabular}{|c|c|c|c|c|c|c|}
\hline $\begin{array}{c}\text { Greenhouse } \\
\text { Gas }\end{array}$ & Season & $\begin{array}{l}\text { Proximal } \\
\text { variables }\end{array}$ & $\begin{array}{l}\text { Ind. p- } \\
\text { value }\end{array}$ & $\begin{array}{c}\text { Model } \\
p- \\
\text { value }\end{array}$ & $\begin{array}{c}\text { Adj. } \\
r^{2}\end{array}$ & Model Equation \\
\hline \multirow{4}{*}{$\begin{array}{c}\text { Carbon } \\
\text { dioxide (mg } \\
\mathrm{CO}_{2^{-}} \\
\mathrm{C} / \mathrm{m}^{2} / \text { day) }\end{array}$} & Winter & Soil \% OM & & 0.004 & 0.58 & $y=187(O M)-438$ \\
\hline & Spring & Soil BD & & 0.08 & 0.2 & $\operatorname{Ln}(y)=2(B D)+6.3$ \\
\hline & Summer & $\begin{array}{l}\text { Soil temp. } \\
\text { Soil \% OM }\end{array}$ & $\begin{array}{c}0.011 \\
0.0496 \\
\end{array}$ & 0.024 & 0.43 & $\begin{array}{l}y=715 \text { (temp.)+ } \\
262(\text { OM) }-13746\end{array}$ \\
\hline & Fall & Soil temp. & & 0.004 & 0.55 & $y=748$ (temp.) -11888 \\
\hline \multirow{3}{*}{$\begin{array}{l}\text { Methane } \\
\left(\mathrm{mg} \mathrm{CH}_{4^{-}}\right. \\
\left.\mathrm{C} / \mathrm{m}^{2} / \text { day }\right)\end{array}$} & Spring & Soil temp. & & 0.067 & 0.28 & $y=0.15$ (temp.) -2.45 \\
\hline & Summer & Soil \% Carbon & & 0.003 & 0.54 & $y=0.19(\%$ carbon $)-1.2$ \\
\hline & Fall & $\begin{array}{l}\text { Soil molar C:N } \\
\text { Soil moisture }\end{array}$ & $\begin{array}{l}0.001 \\
0.005\end{array}$ & 0.004 & 0.75 & $\begin{array}{c}y=0.03(C: N)+ \\
1.9(\text { moisture })-1.8\end{array}$ \\
\hline
\end{tabular}

$\mathrm{BD}=$ bulk density $\left(\mathrm{g} / \mathrm{m}^{3}\right) ;$ Soil moisture $=\mathrm{g}$ water $/ \mathrm{g}$ dry soil; Soil temperature $={ }^{\circ} \mathrm{C}$

Ind. $\mathrm{P}$-value $=\mathrm{p}$-values for individual explanatory variables in a multiple regression model. 

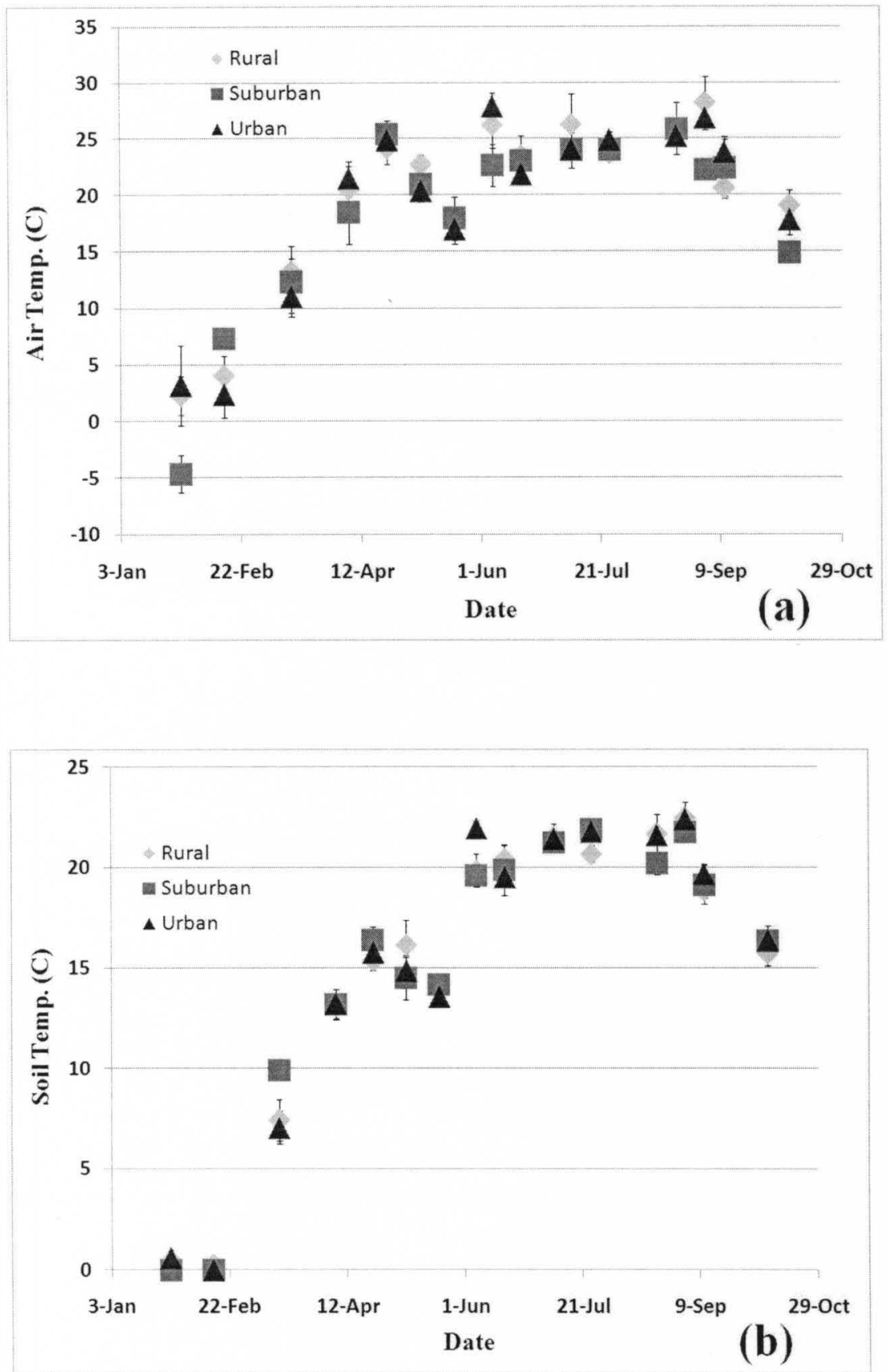

Figure 4-1a-b: Air temperature and soil temperature taken during twice-monthly gas sampling visits in urban, suburban, and rural riparian sites in 2008. Soil temperature was taken in two locations within each site to five, ten, and fifteen $\mathrm{cm}$ depths and averaged to obtain a representative soil temperature through the soil column. Air temperature was taken between the two soil temperature locations. Points are means of five urban, three suburban, and four rural sites with standard error bars. Urban subcatchments contain $\geq$ $30 \%$ impervious surface cover, rural subcatchments contain $\leq 10 \%$ impervious surface cover, and suburban subcatchments contain values in between 10 and $30 \%$ impervious surface cover. 


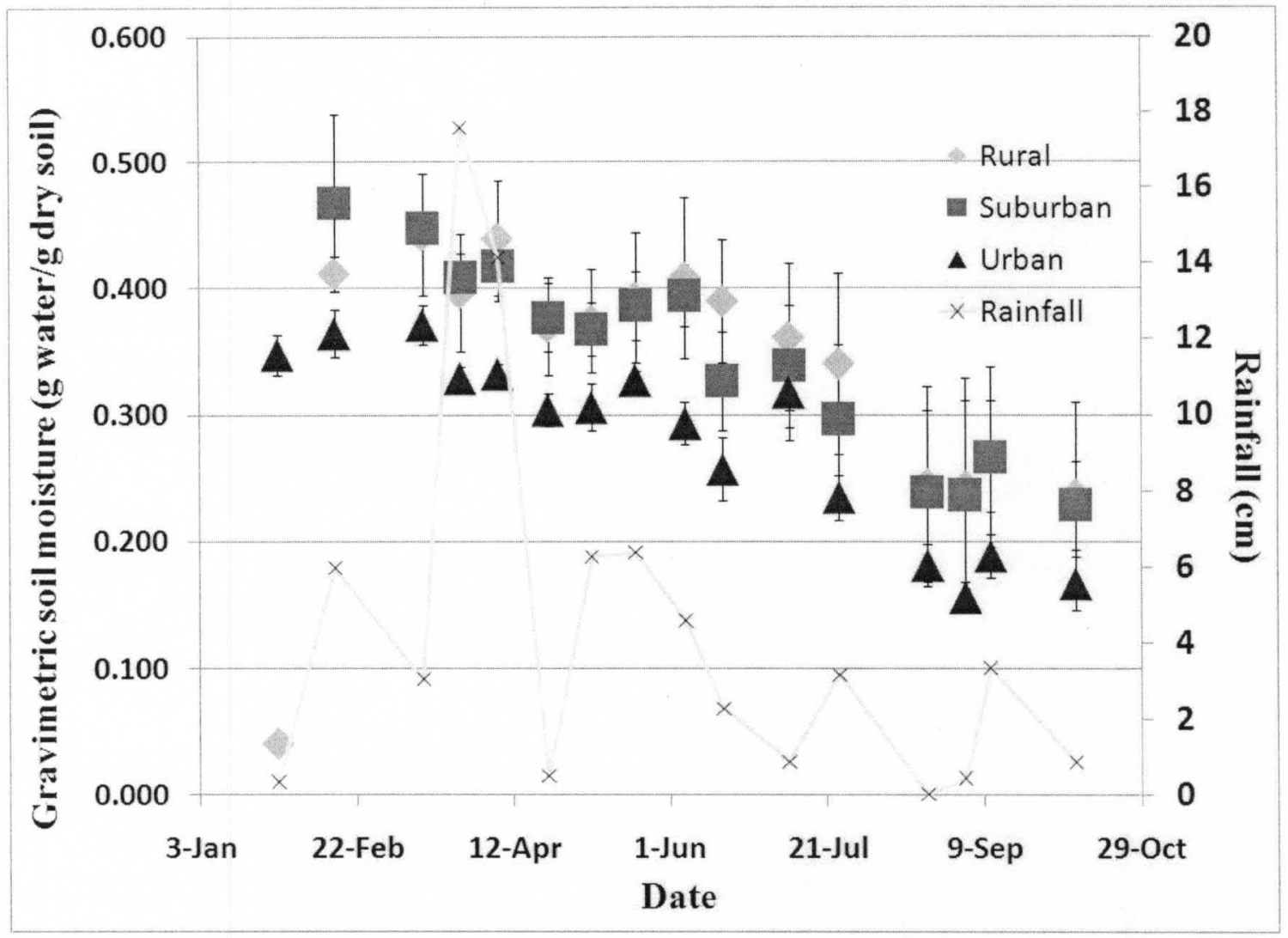

Figure 4-2: Gravimetric soil moisture in the upper 15-cm soil horizon in urban, suburban, and rural riparian sites in 2008. Points are means of five urban, three suburban, and four rural sites with standard error bars. Rainfall was determined from a single location monitored by the Louisville Metropolitan Sewer District within Goose and Beargrass creek watersheds and represents total ppt. monthly in January and February and every two weeks from March to October. Urban subcatchments contain $\geq 30 \%$ impervious surface cover, rural subcatchments contain $\leq 10 \%$ impervious surface cover, and suburban subcatchments contain values in between 10 and $30 \%$ impervious surface cover. 


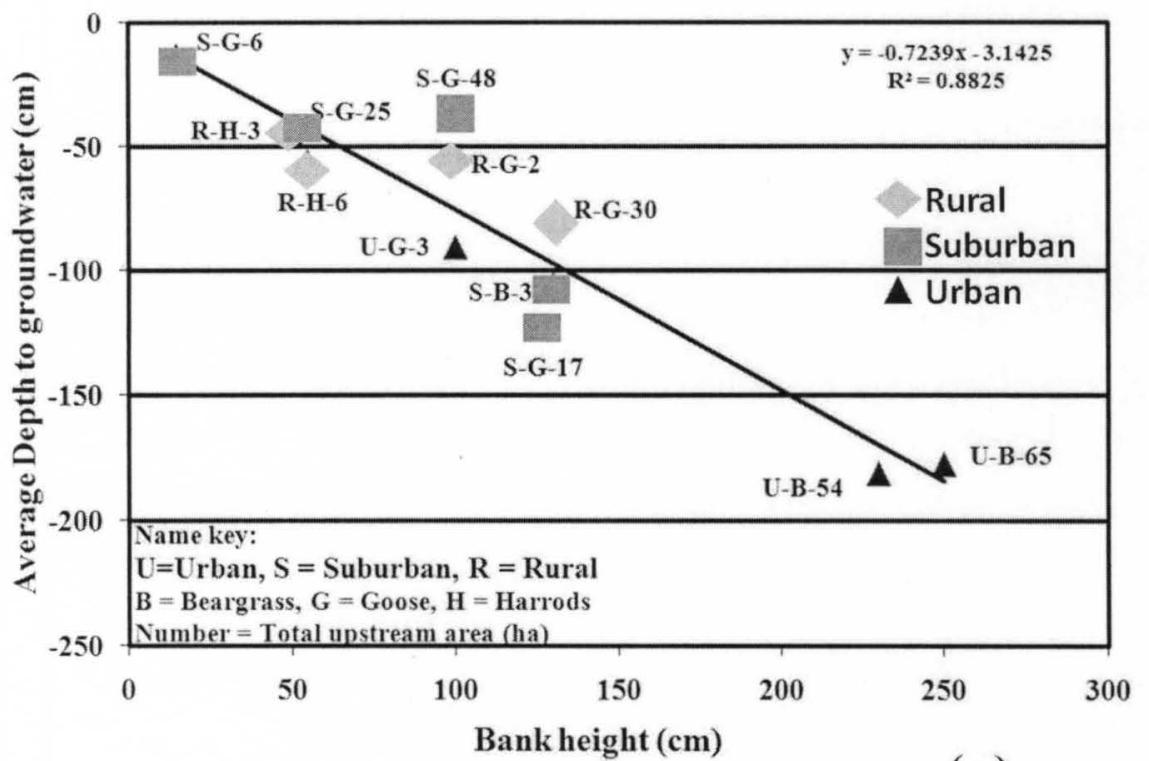

(a)

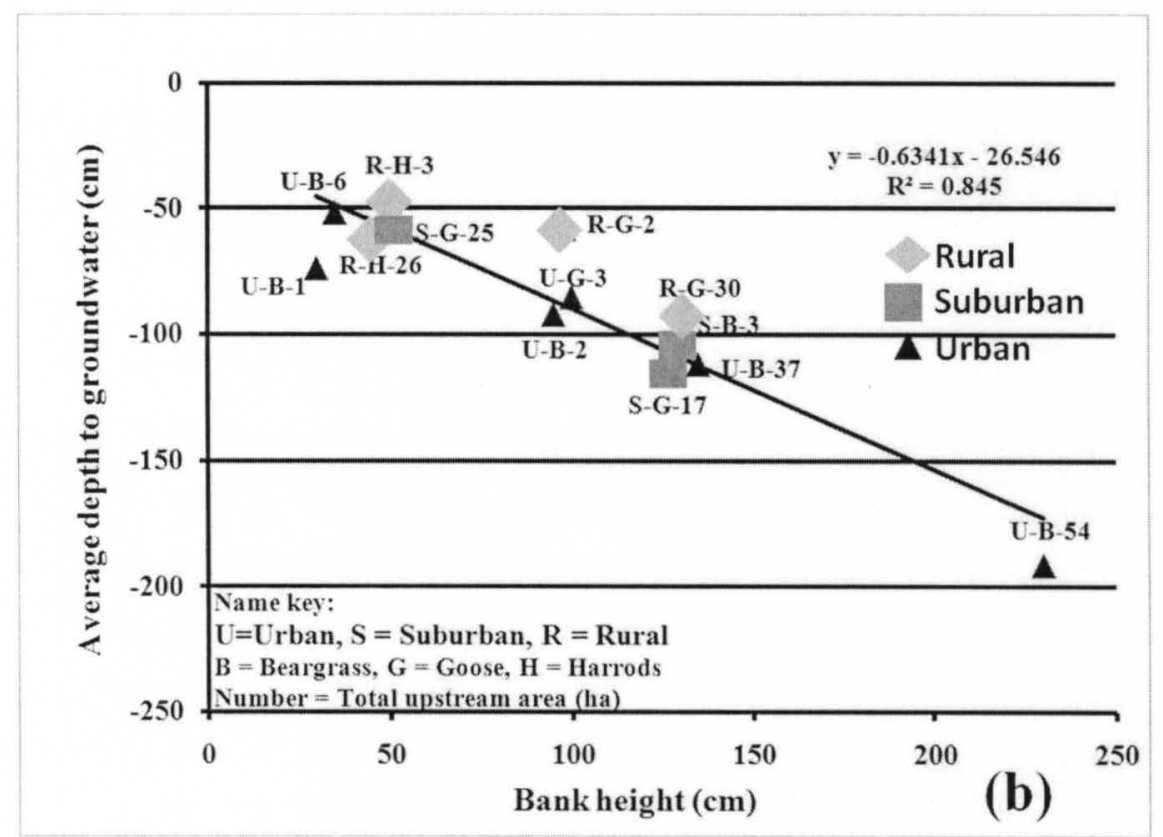

Figure 4-3a-b: Nearstream depth to groundwater (water table) in relation to bank height in years 2007 (a) and 2008 (b). Depth to groundwater was measured twice a month in riparian groundwater wells in urban, suburban, and rural sites from November 2006 to October 2007 (a) and from November 2007 to October 2008 (b). Urban subcatchments contain $\geq 30 \%$ impervious surface cover, rural subcatchments contain $\leq 10 \%$ impervious surface cover, and suburban subcatchments contain values in between 10 and $30 \%$ impervious surface cover. 


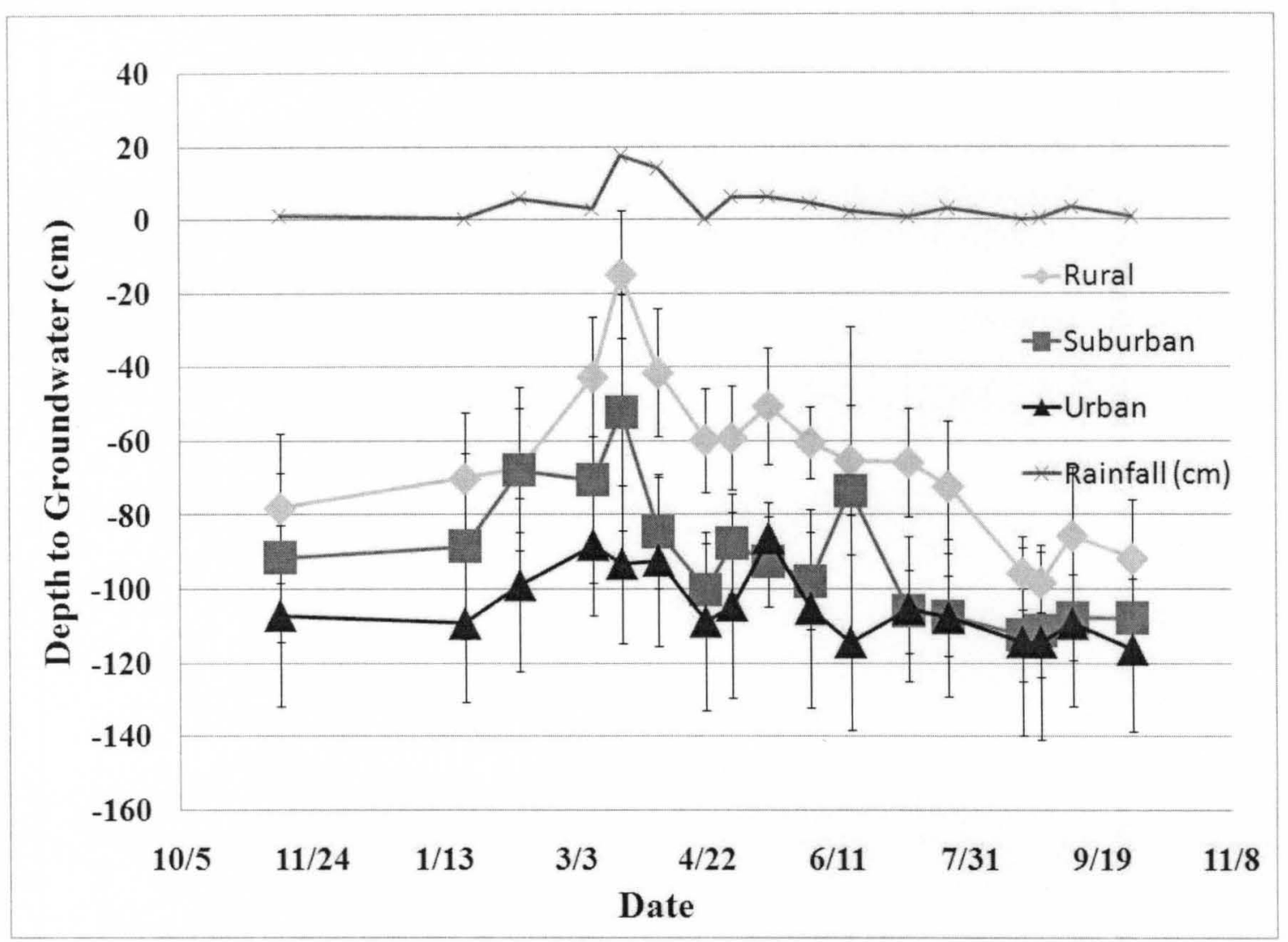

Figure 4-4: Nearstream depth to groundwater (water table) from November 2007 to October 2008. Depth to groundwater was measured once or twice a month in riparian groundwater wells in urban, suburban, and rural sites based on subcatchment $\%$ impervious surface. Points are means of five urban, three suburban, and three rural sites with standard error bars. Rainfall was determined from a single location monitored by the Louisville Metropolitan Sewer District within Goose and Beargrass creek watersheds and represents total ppt. monthly in January and February and every two weeks from March to October. Urban subcatchments contain $\geq 30 \%$ impervious surface cover, rural subcatchments contain $\leq 10 \%$ impervious surface cover, and suburban subcatchments contain values in between 10 and $30 \%$ impervious surface cover. Repeated-measures ANOVA revealed a significant time effect within groups $(\mathrm{p}=<0.0001)$, but no significant between-group effects were observed $(\mathrm{p}=0.44)$. 


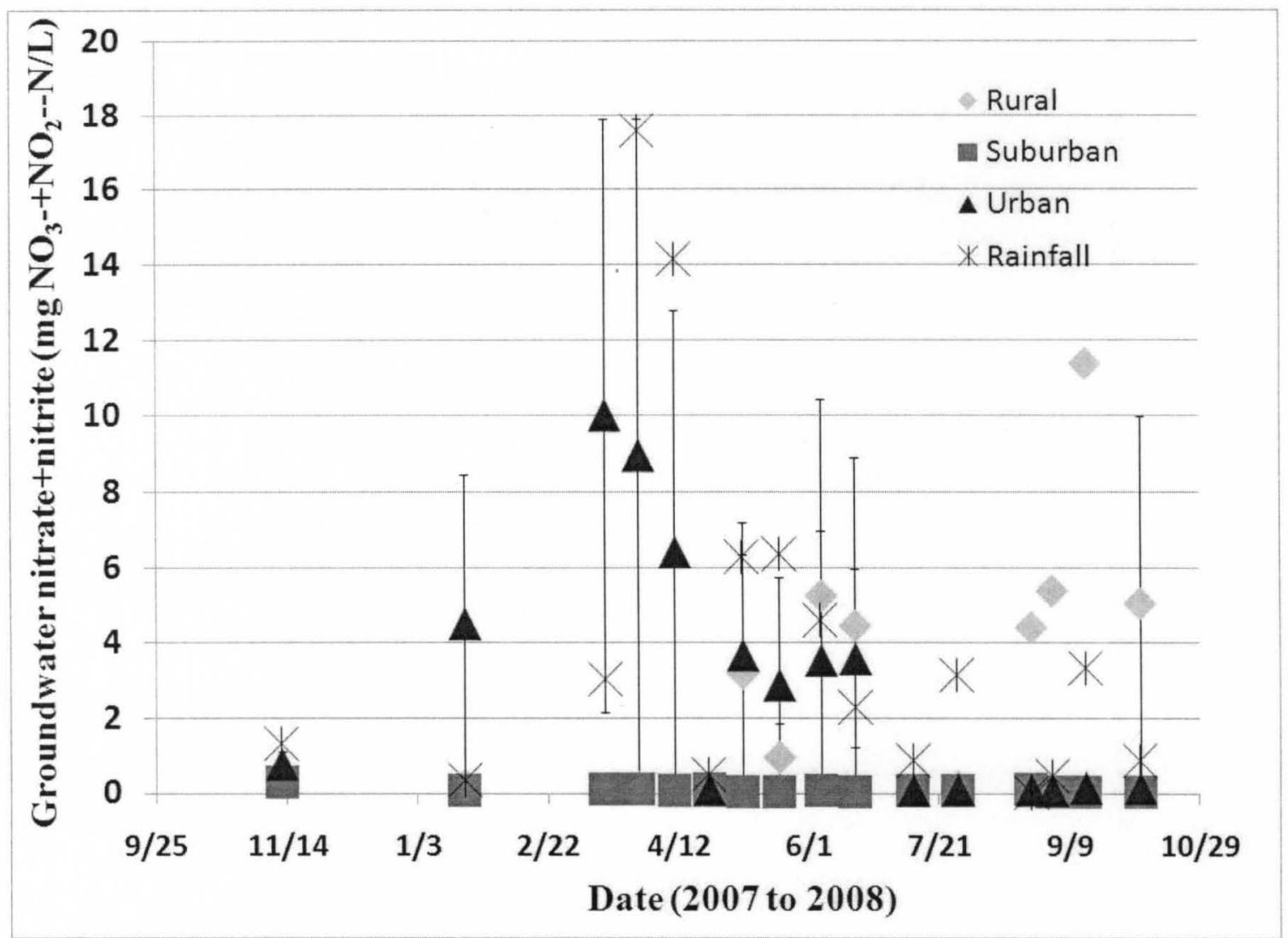

Figure 4-5: Mean nitrate+nitrite- $\mathrm{N}$ nearstream riparian groundwater concentrations in urban, suburban, and rural subcatchments. Nitrate+nitrite- $\mathrm{N}$ values are means from five or six urban, 3 suburban, and 3 rural sites. Rainfall was determined from a single location monitored by the Louisville Metropolitan Sewer District within Goose and Beargrass creek watersheds and represents total ppt. monthly in January and February and every two weeks from March to October. Urban subcatchments contain $\geq 30 \%$ impervious surface cover, rural subcatchments contain $\leq 10 \%$ impervious surface cover, and suburban subcatchments contain values in between 10 and 30\% impervious surface cover. 


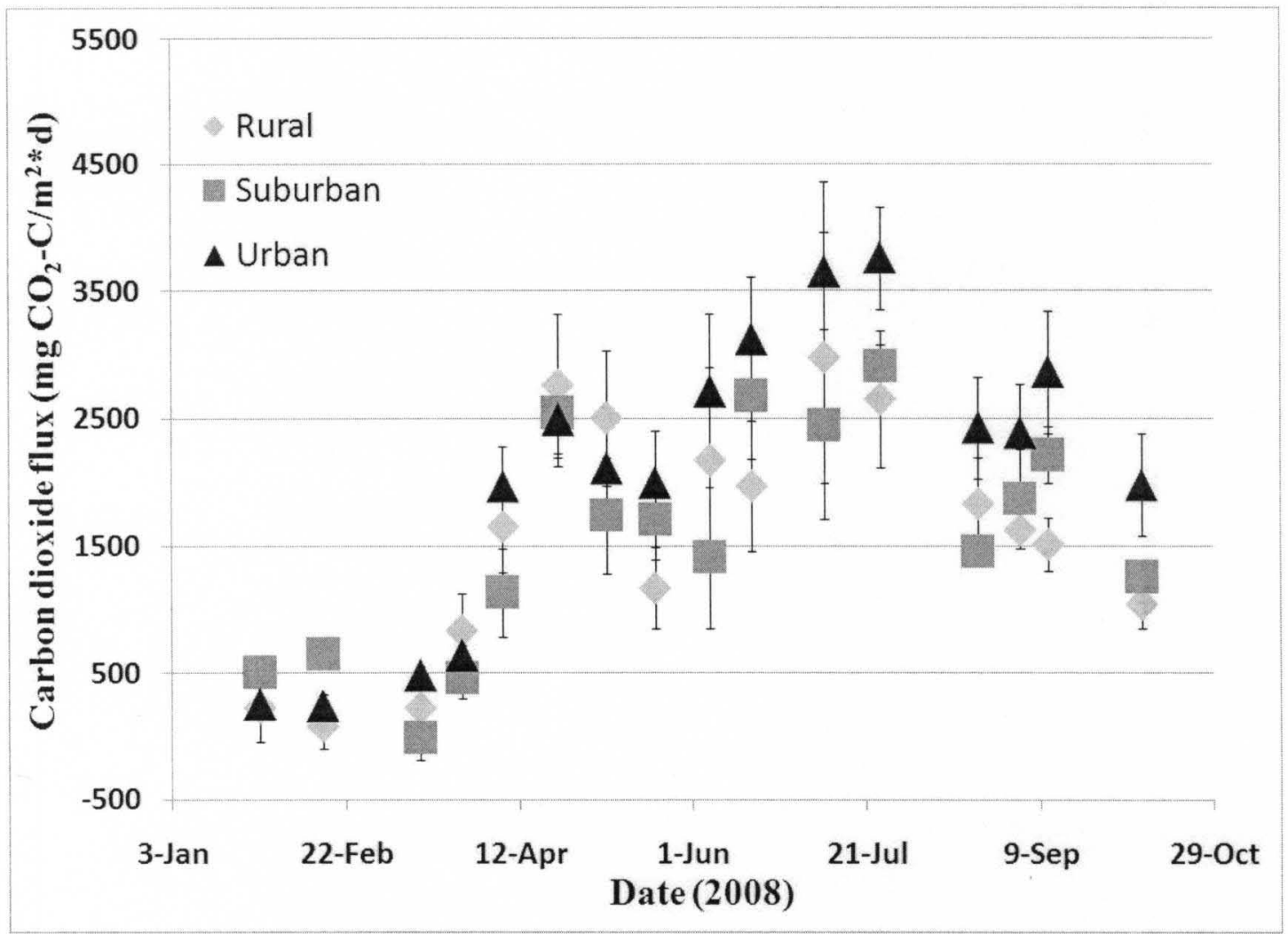

Figure 4-6: Mean ( $\_$SE) riparian $\mathrm{CO}_{2}$ flux between January and October 2008 averaged for sites in rural, suburban, and urban subcatchments. Points are means of five or six urban, three suburban, and four rural values. Urban subcatchments contain $\geq 30 \%$ impervious surface cover, rural subcatchments contain $\leq 10 \%$ impervious surface cover, and suburban subcatchments contain values in between 10 and $30 \%$ impervious surface cover. 


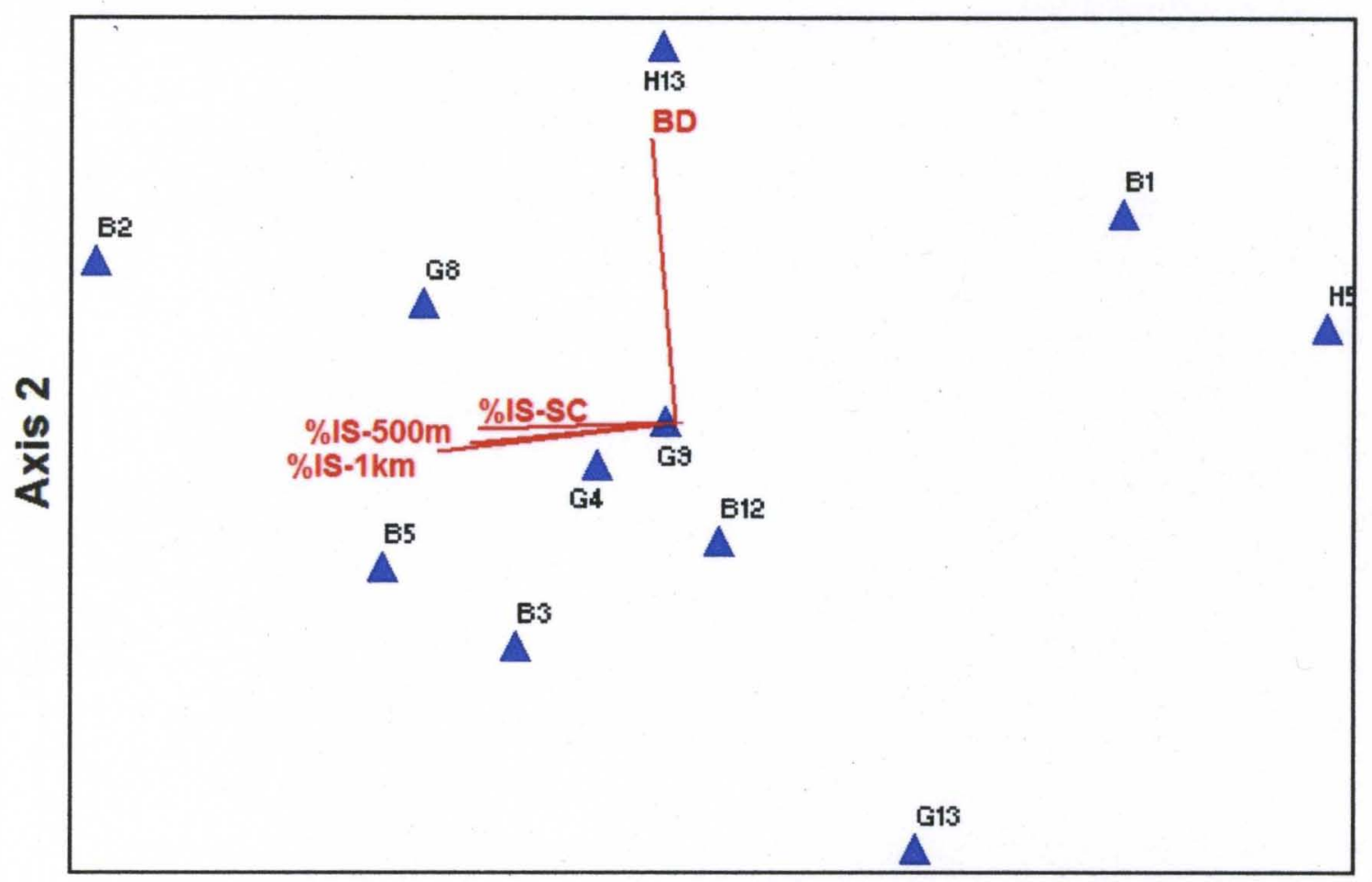

Axis 1

Figure 4-7: Principal components analysis (PCA) of sites and monthly $\mathrm{CO}_{2}$ fluxes. Sites are arranged according to mean annual $\mathrm{CO}_{2}$ flux along axis 1 , with increasing $\mathrm{CO}_{2}$ emissions from right to left. Axis 2 separation among sites was primarily explained by soil bulk density, which increases from bottom to top of the graph. Vectors represent site and soil properties that have a coefficient of determination $\geq 0.3$ with at least one axis. Visible site and soil variables are $\mathrm{BD}=$ bulk density, $\% \mathrm{IS}=$ percent impervious surface at two site radius buffers - 500-meters $(500 \mathrm{~m})$ and 1-kilometer $(1 \mathrm{~km})$ - and within the subcatchment (SC) in which the site is located. 


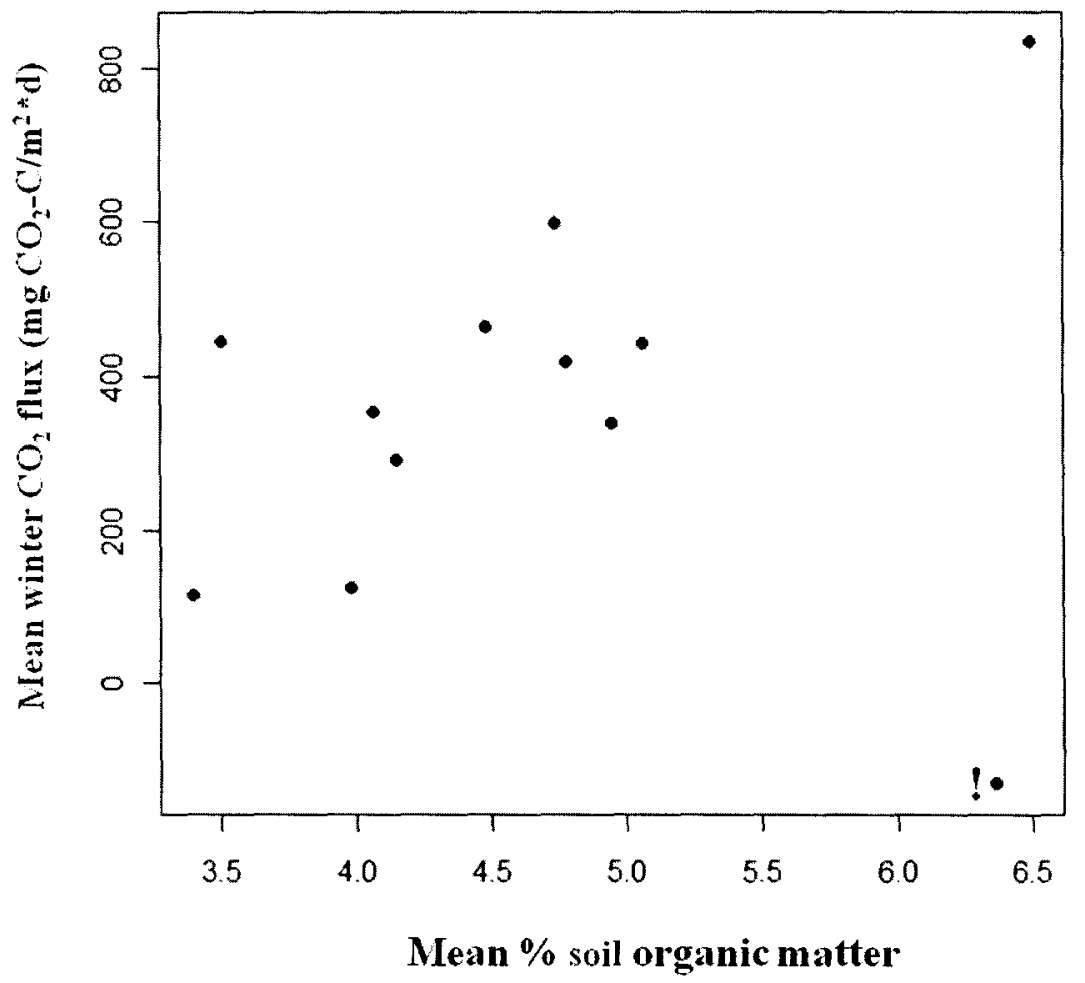

Figure 4-8: Relationship between riparian winter $\mathrm{CO}_{2}$ flux and \% soil organic matter (OM). Carbon dioxide fluxes and \% soil OM were averaged for each site for cold weather months (January, February, and March) and entered into a linear regression model ( $p=$ 0.004 , adj. $\mathrm{r}^{2}=0.58$ ). The site marked with an "!" was determined to be an outlier as described in Methods (this chapter). 


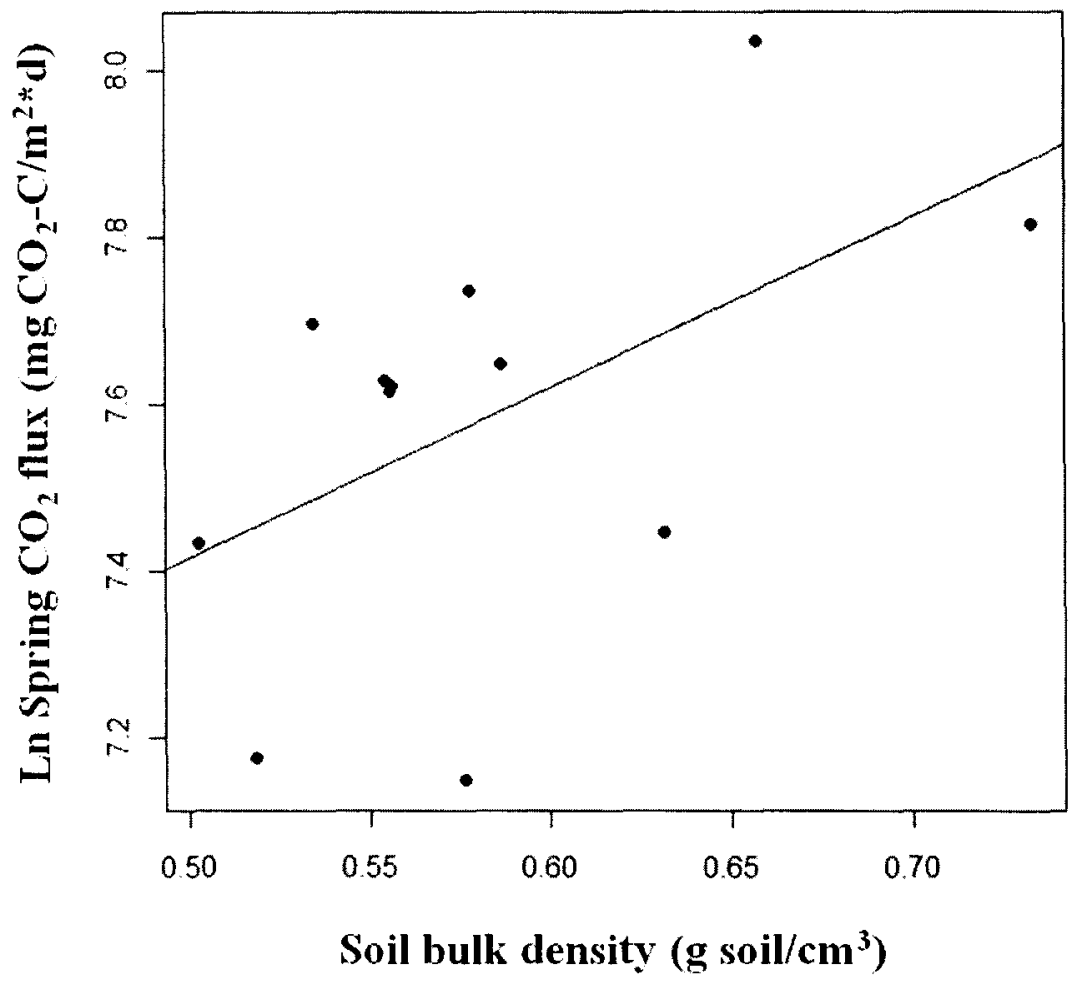

Figure 4-9: Relationship between riparian spring $\mathrm{CO}_{2}$ flux and soil bulk density (BD). Carbon dioxide flux was averaged for each site for spring months (April and May). BD was determined from October 2008 soil samples. $\mathrm{BD}$ in this range exhibited a positive relationship with $\mathrm{CO}_{2}$ flux $\left(\mathrm{p}=0.081 ; \mathrm{r}^{2}=0.20\right)$. 


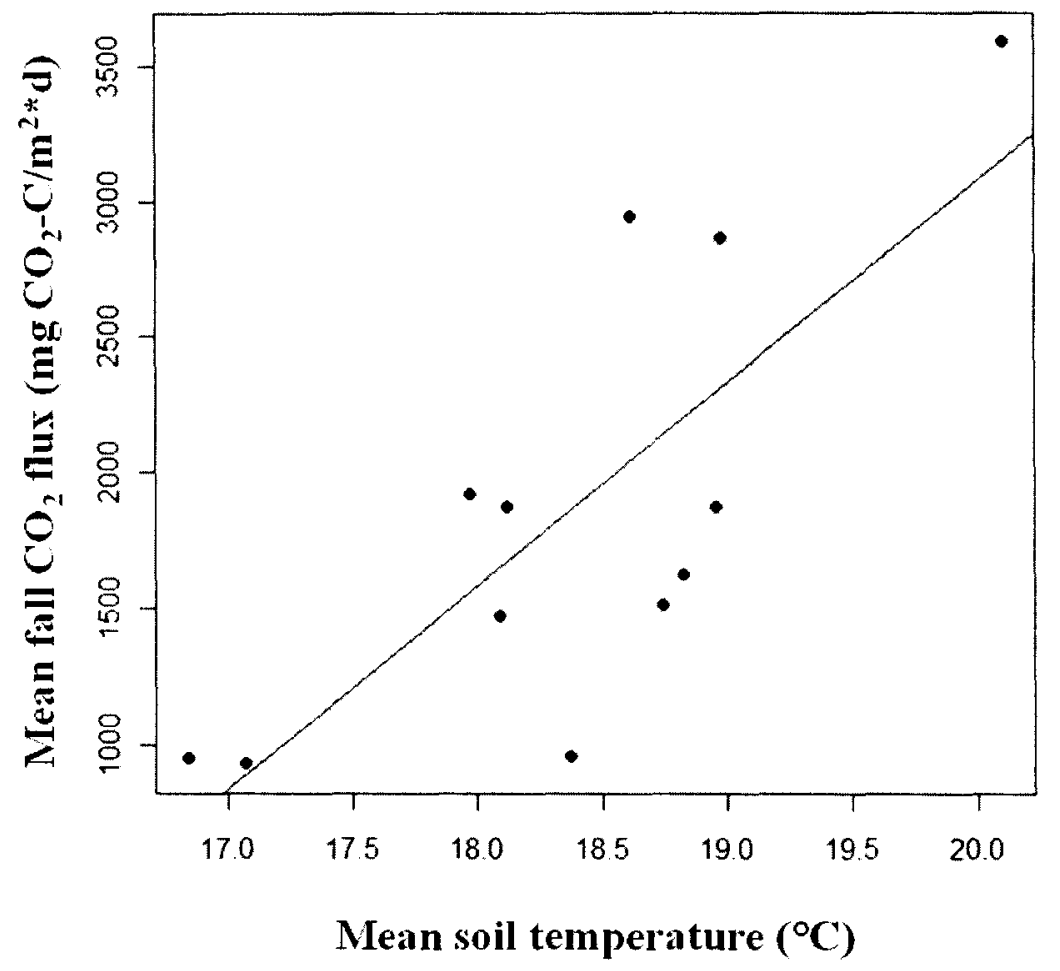

Figure 4-10: Increasing mean fall $\mathrm{CO}_{2}$ flux rates with increasing mean soil temperature among riparian sites. Each point is the average of three site measurements for each variable taken during September and October 2008. The adjusted $r^{2}$ was 0.56 and the pvalue was 0.004 . 


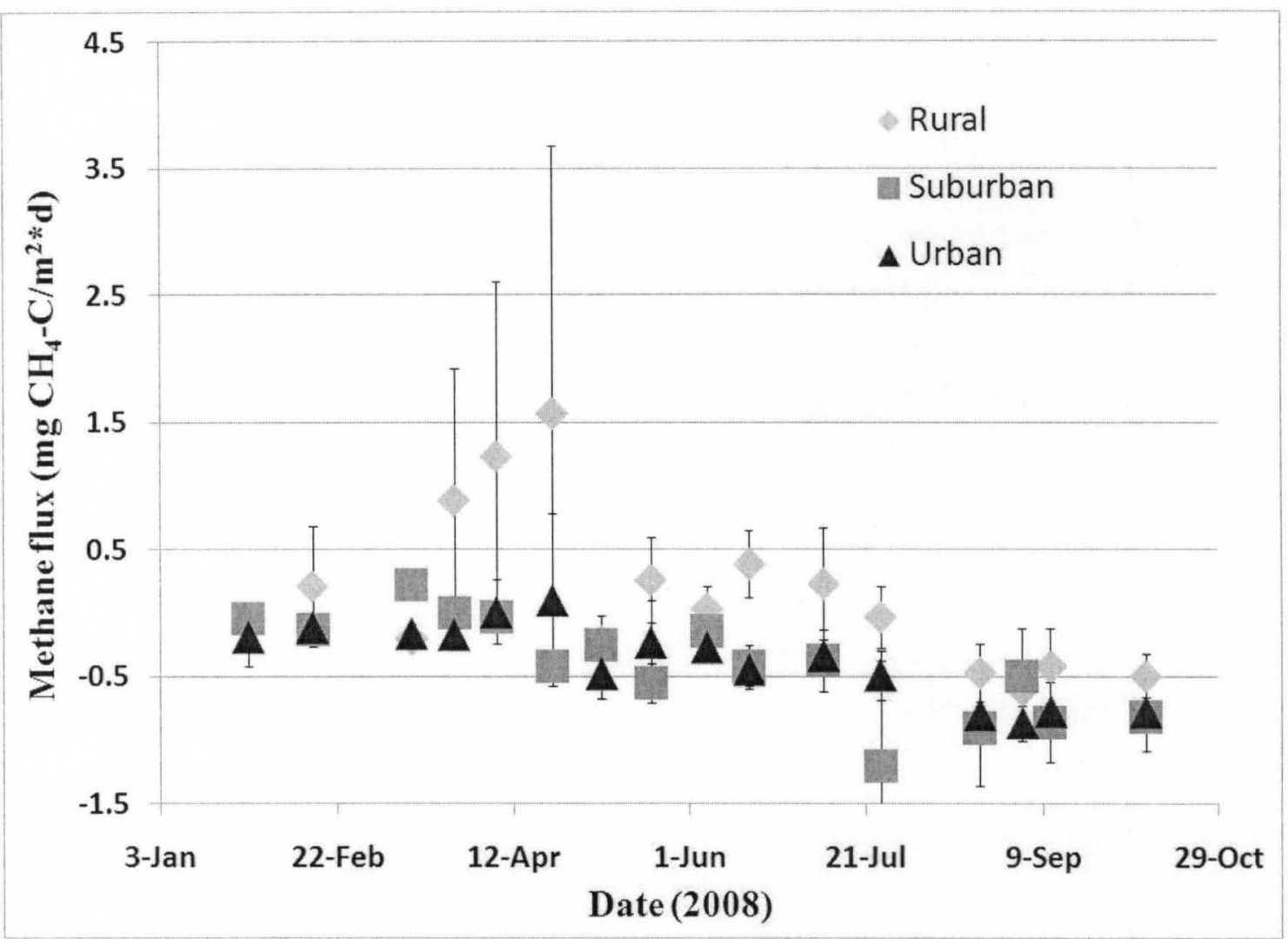

Figure 4-11: Mean $( \pm \mathrm{SE})$ riparian $\mathrm{CH}_{4}$ fluxes from January to October 2008 averaged for sites in rural, suburban, and urban subcatchments. Points are means of five or six urban, three suburban, and four rural values. Values above zero indicate net $\mathrm{CH}_{4}$ efflux from the soil. Values below zero indicate net $\mathrm{CH}_{4}$ uptake. Urban subcatchments contain $\geq$ $30 \%$ impervious surface cover, rural subcatchments contain $\leq 10 \%$ impervious surface cover, and suburban subcatchments contain values in between 10 and $30 \%$ impervious surface cover. 


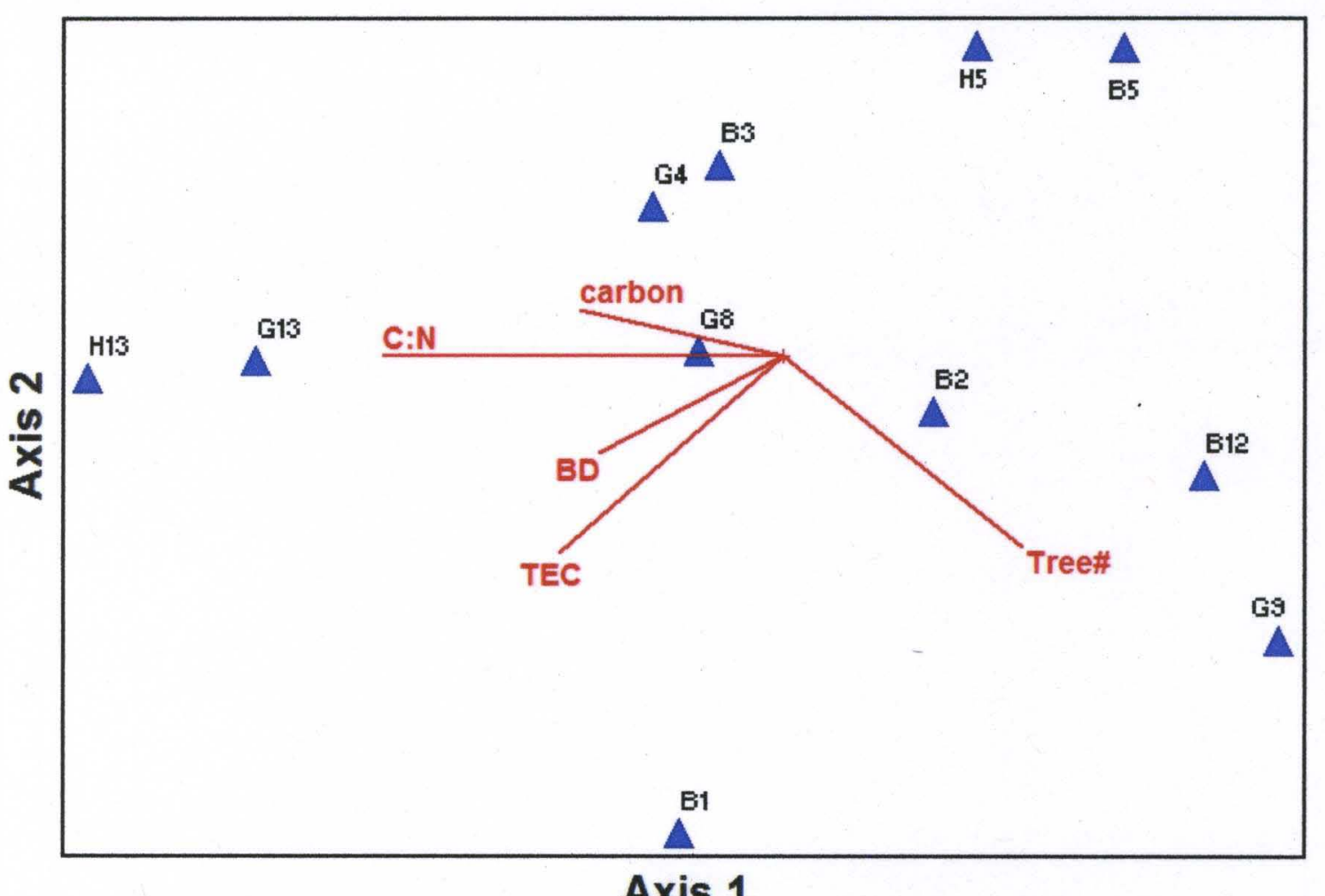

Figure 4-12: Principal components analysis (PCA) of sites and mean monthly $\mathrm{CH}_{4}$ fluxes from January to October 2008. Sites are arranged according to monthly average $\mathrm{CH}_{4}$ flux along axis 1, with increasing $\mathrm{CH}_{4}$ uptake from left to right. Vectors represent site and soil properties that have a coefficient of determination $\geq 0.3$ with at least one axis. Soil variables are $\mathrm{TEC}=$ total exchange capacity, $\mathrm{BD}=$ bulk density, carbon $=\%$ carbon, $\mathrm{C}: \mathrm{N}$ $=$ molar $\mathrm{C}: \mathrm{N}$ ratio. The site variable Tree\# represents average number of trees $>1-\mathrm{m}$ high and $\geq 2.54-\mathrm{cm}$ diameter breast height within five meters of the area of $\mathrm{CH}_{4}$ flux determination. 


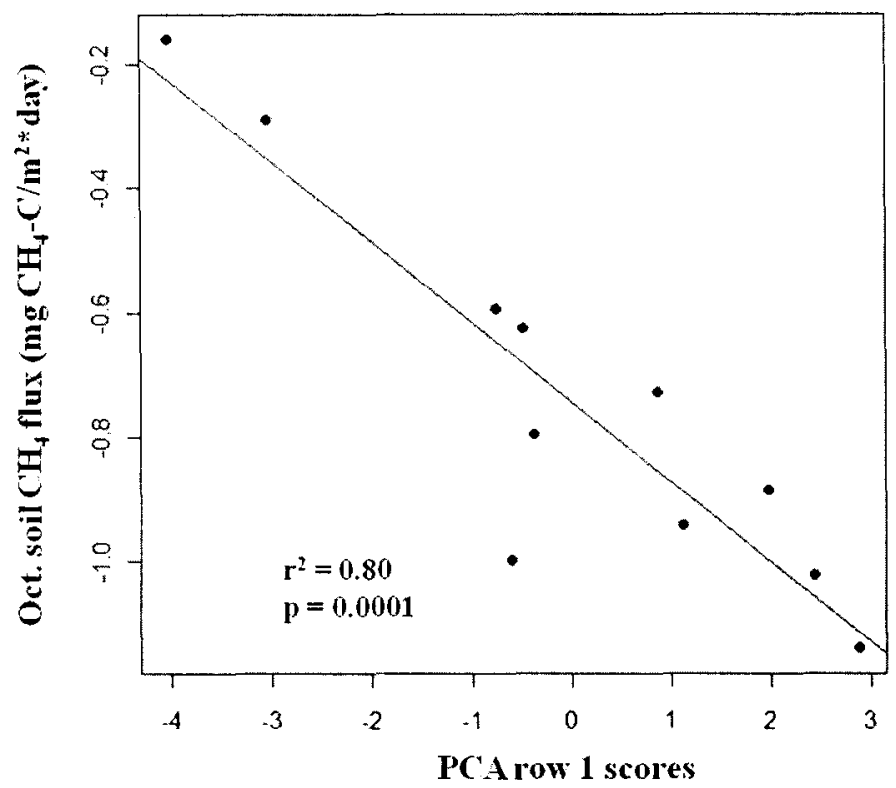

(a)

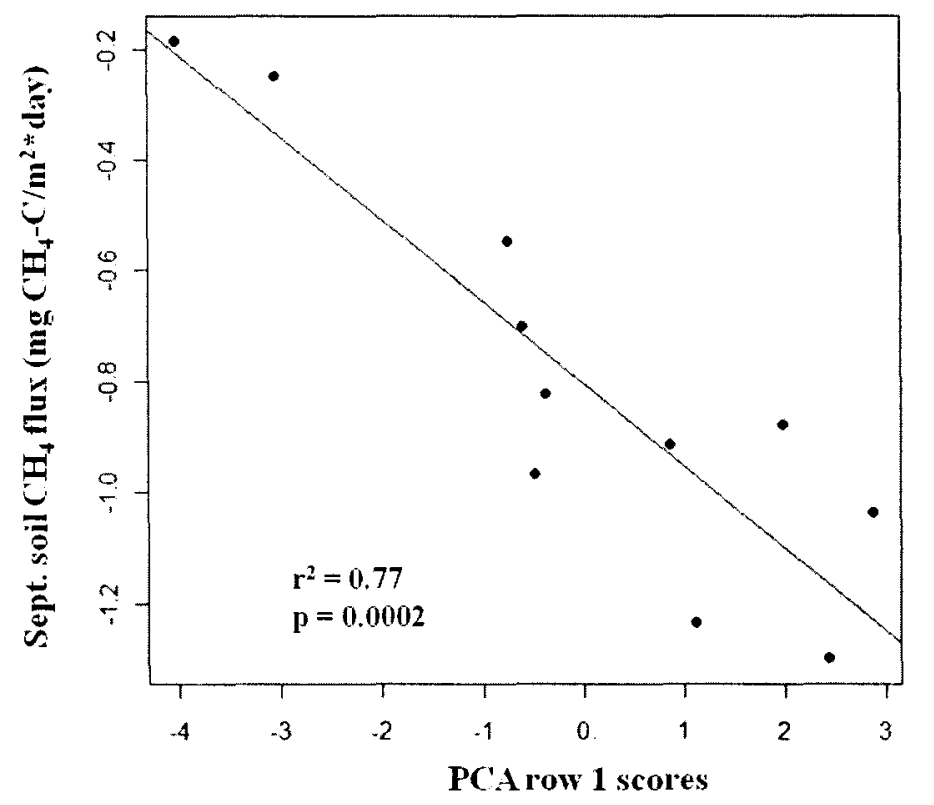

(b)

Figure 4-13a-b: Mean monthly methane flux rates at each site from 2008 in relation to PCA row 1 scores from Fig. 4-12 for the months October (a) and September (b). Site $\mathrm{CH}_{4}$ fluxes occur along a linear gradient from left to right on $\mathrm{x}$-axis from low methane uptake to high methane uptake in September and October. The linear pattern began to dissipate in August $\left(r^{2}=0.55\right)$ and the gradient becomes non-linear in July as well as in months prior through January (not shown). 


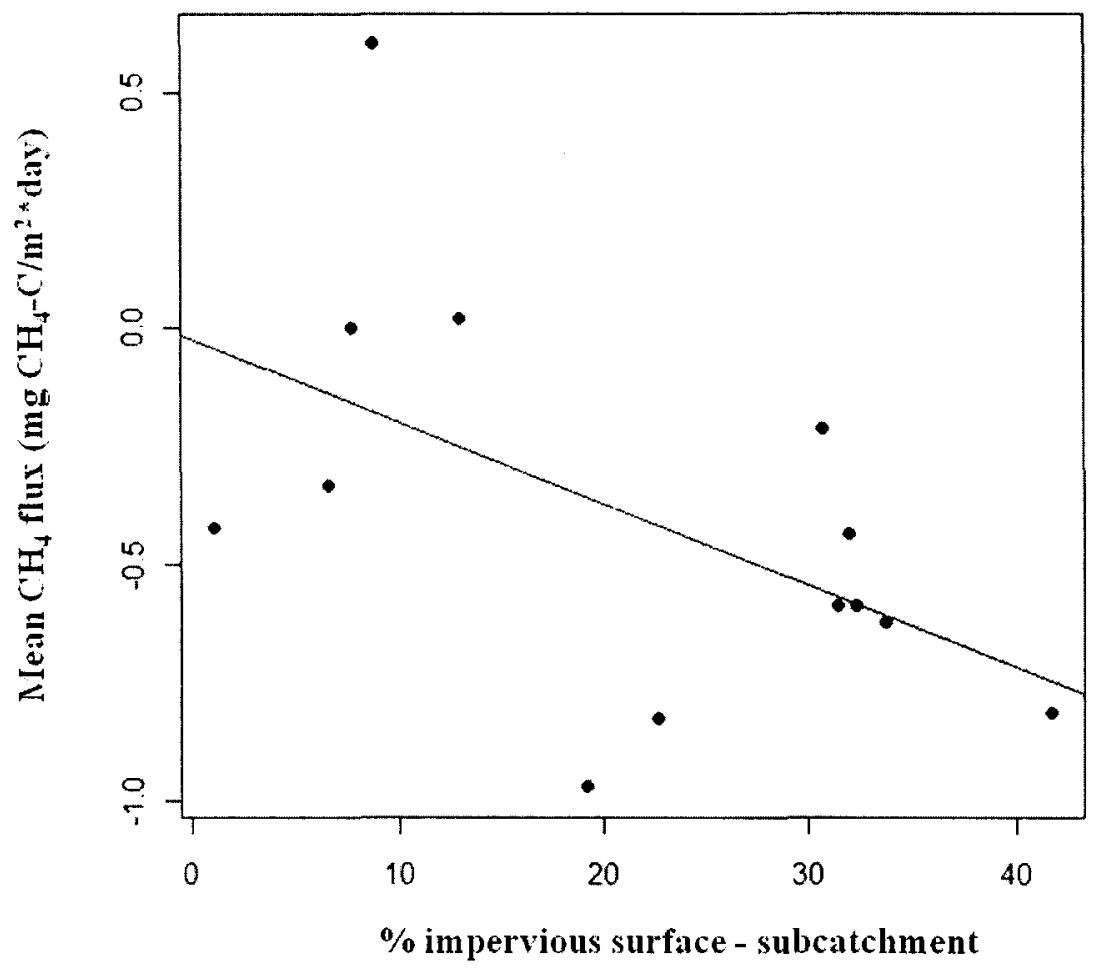

Figure 4-14: Changes in mean summer $\mathrm{CH}_{4}$ flux in relation to subcatchment \%IS. Site methane fluxes and soil moisture values were averaged over June, July, and August 2008. Soil uptake of $\mathrm{CH}_{4}$ increased with increasing \% $\mathrm{IS}\left(\mathrm{p}=0.06\right.$, adj. $\mathrm{r}^{2}=0.21, \mathrm{y}=0.02 \mathrm{x}-$ 0.03 ). Values below zero indicate mean $\mathrm{CH}_{4}$ uptake of by soils. 


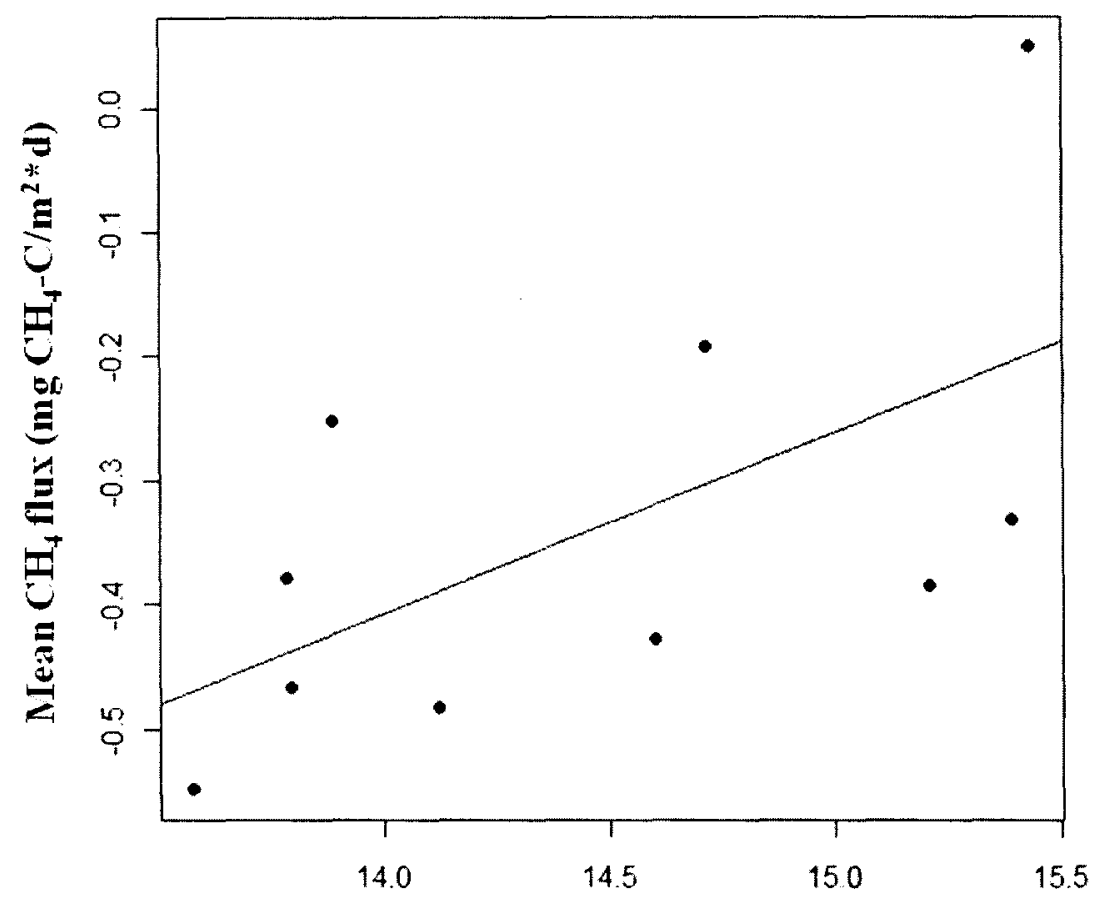

Mean soil temperature $\left({ }^{\circ} \mathrm{C}\right)$

Figure 4-15: Changes in mean spring $\mathrm{CH}_{4}$ flux with increasing mean soil temperature among sites averaged over April and May $2008\left(p=0.067\right.$; adj. $\mathrm{r}^{2}=0.28 ; \mathrm{y}=0.15 \mathrm{x}-$ 2.45). Methane fluxes and soil temperature were taken on two separate dates each month. Negative fluxes indicate soil uptake from the atmosphere. Two sites (UB1 \& RG7) were removed from this analysis because they exhibited high levels of net $\mathrm{CH}_{4}$ efflux. 


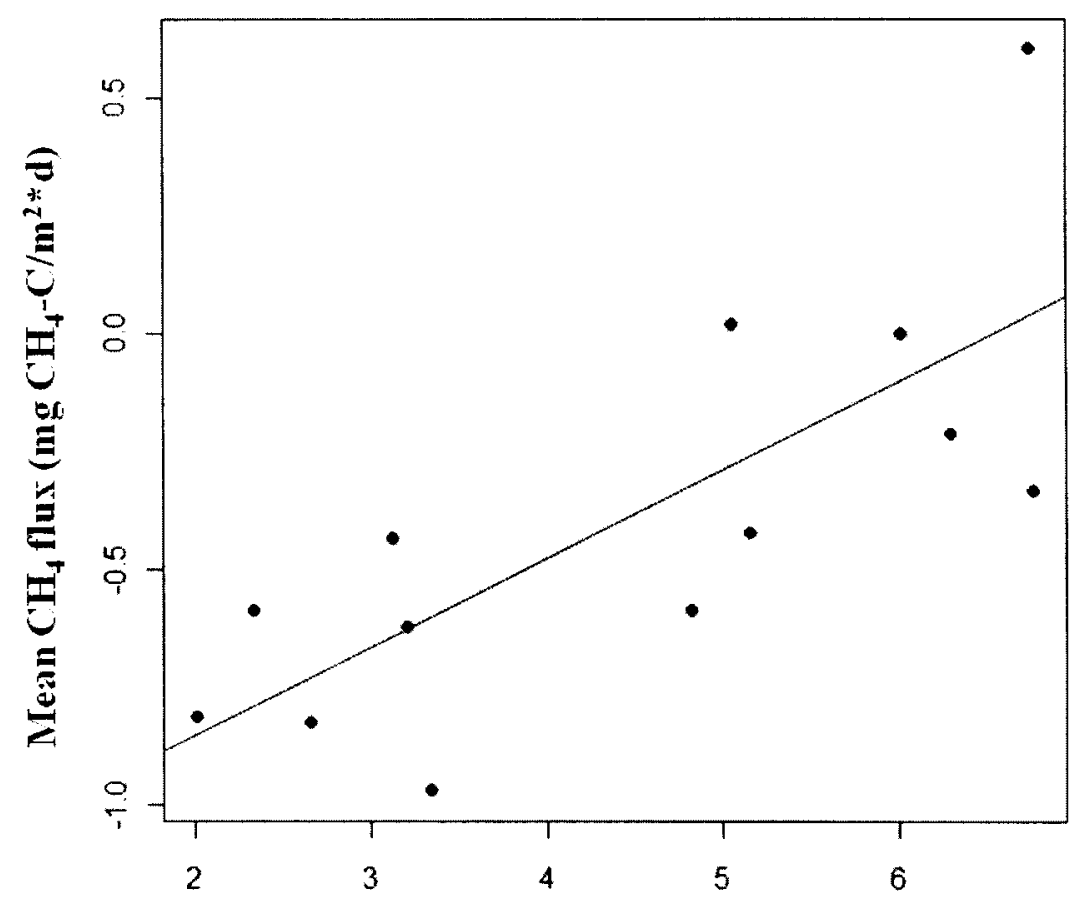

Soil \% carbon

Figure 4-16: Summer mean net $\mathrm{CH}_{4}$ uptake (values less than zero) increases (values from left to right) with decreasing soil \% carbon. Five flux measurements, taken in June, July, and August, 2008, were averaged to obtain mean flux. Soil \% carbon was determined for each site from a single sample taken in October 2008. The p-value was 0.003 and the adjusted $\mathrm{r}^{2}$ was $0.5(\mathrm{y}=0.19 \mathrm{x}-1.2)$. 


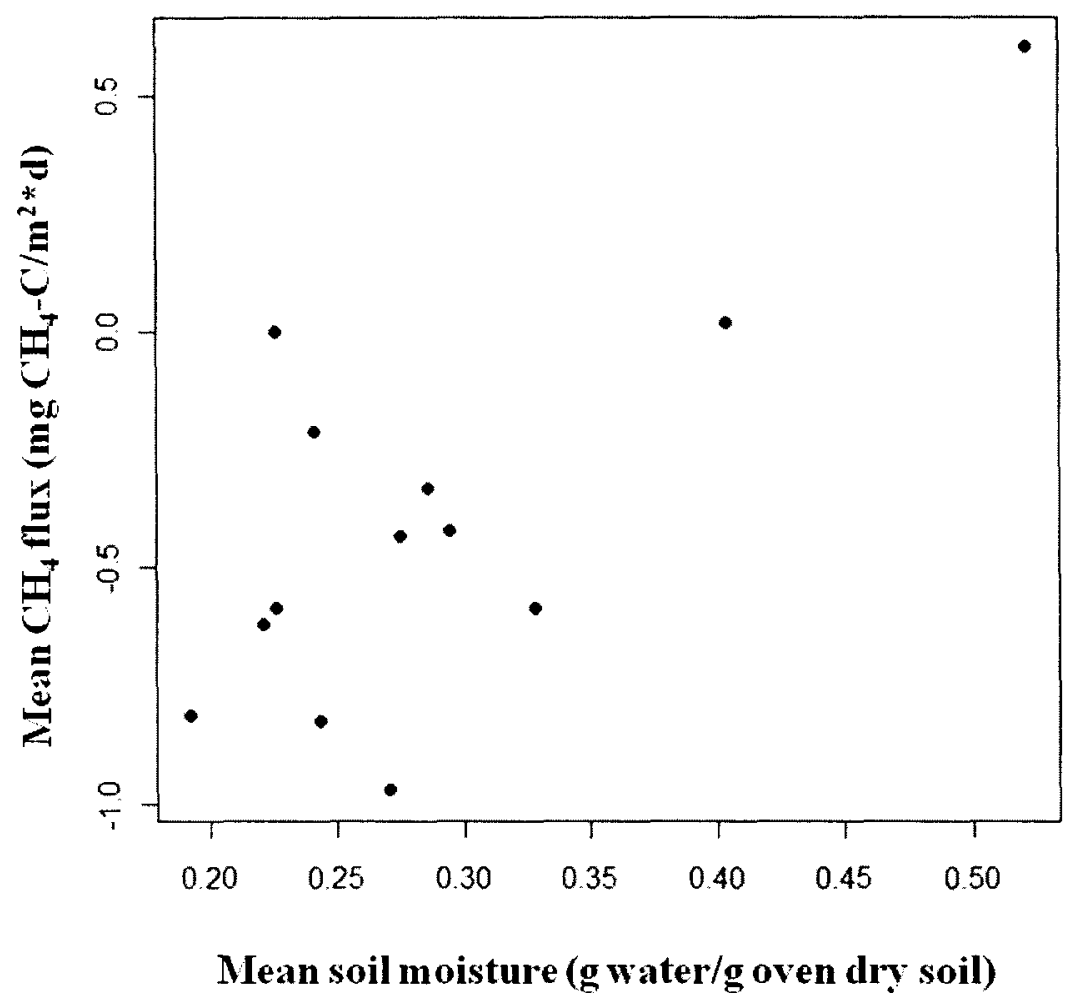

Figure 4-17: Variation in site $\mathrm{CH}_{4}$ flux with soil moisture. Site methane fluxes and soil moisture values were averaged over June, July, and August 2008. Methane fluxes and soil moisture were measured twice monthly except August, when one sample was collected. 


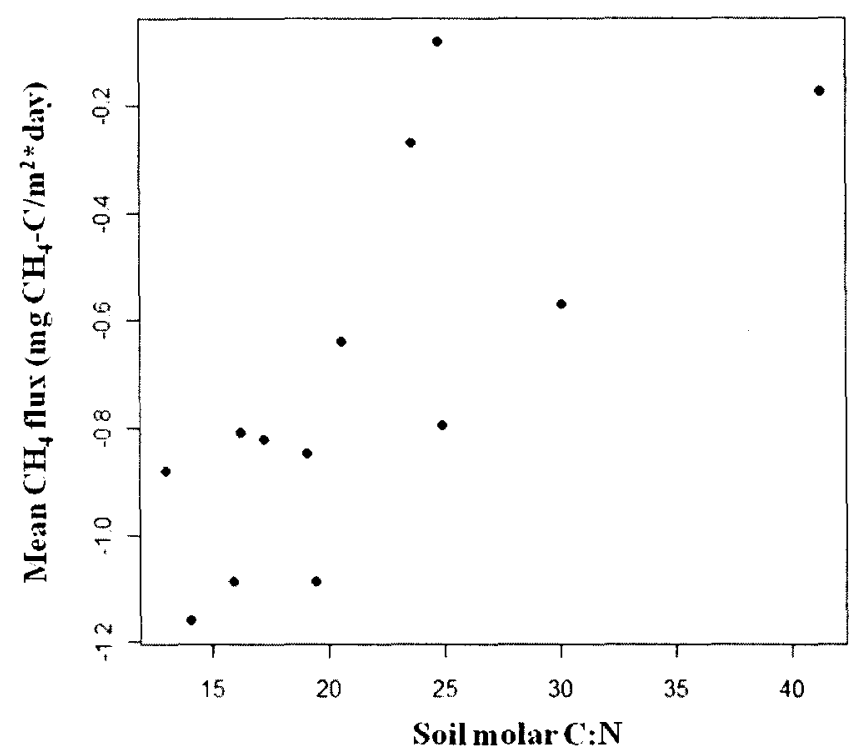

(a)

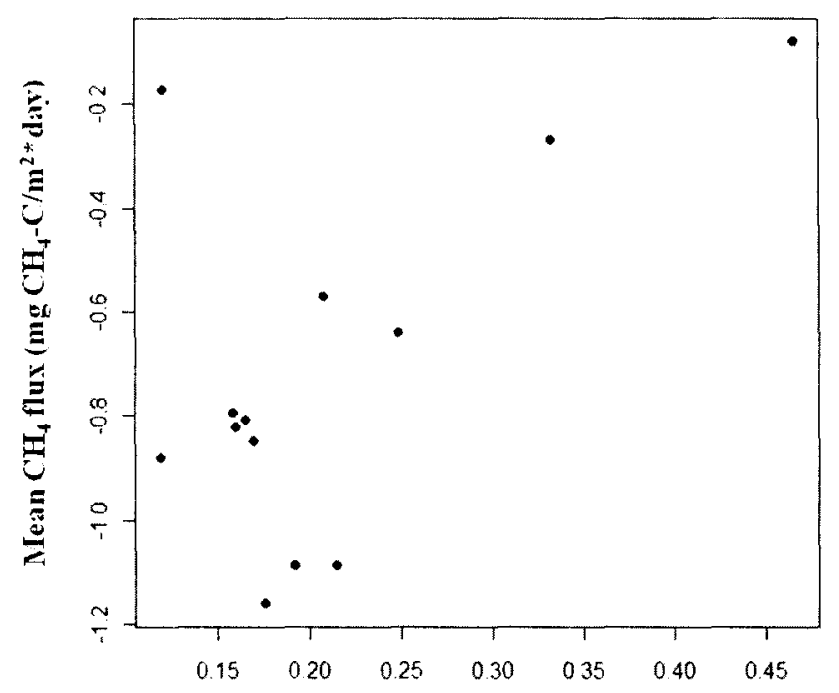

Soil moisture (g water/g oven dry soil)

(b)

Figure 4-18a-b: Relationships between mean fall $2008 \mathrm{CH}_{4}$ flux and soil molar C:N ratios (a) and gravimetric soil moisture (b). Methane fluxes from September and October were averaged for each site. Soil C:N ratios were determined from soil samples collected in October. Gravimetric soil moisture was determined to a depth of $15-\mathrm{cm}$ adjacent to each gas sampling chamber and averaged for the site each time samples were collected. A multiple regression was used to assess relationships of these two variables with $\mathrm{CH}_{4}$ flux. The overall adjusted coefficient of determination for the model was 0.75 , with a p-value $=0.0004$. Individual $p$-values for each variable and the equation can be found in Table 46. 


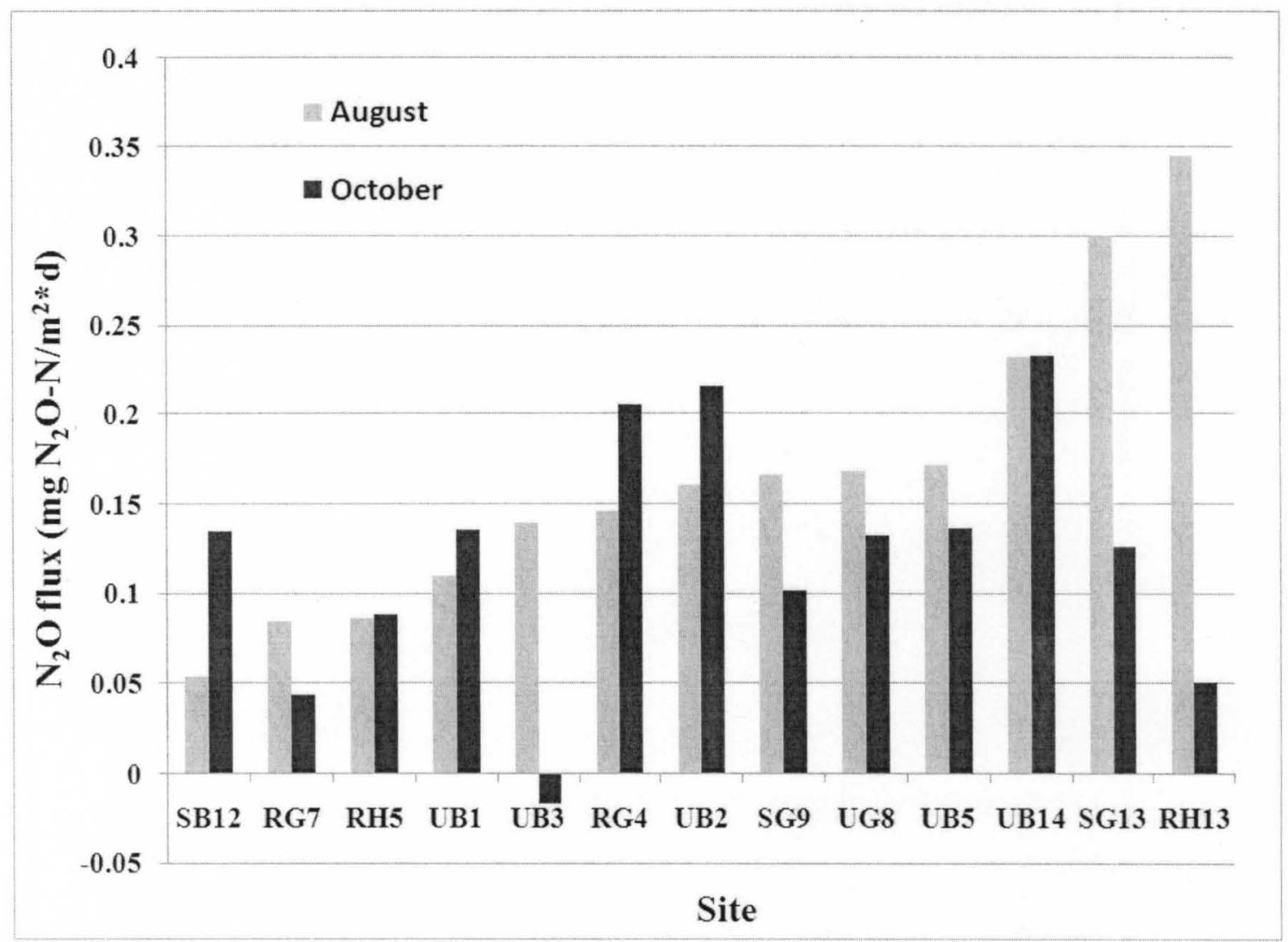

Figure 4-19: Nitrous oxide flux from static chambers along riparian sites in three watersheds spanning an urban-to-rural gradient. Sites were ordered from low to high $\mathrm{N}_{2} \mathrm{O}$ flux from left to right. Site names reflect the urban-to-rural gradient and the watershed in which they are located. The first letter in the site name reflects the urban-to-rural gradient, where $\mathrm{R}=$ rural, $\mathrm{S}=$ suburban, and $\mathrm{U}=$ urban based on impervious surface at the subcatchment scale.The second letter reflects the watershed in which the site is located, where $\mathrm{B}=$ Beargrass, $\mathrm{G}=\mathrm{Goose}$, and $\mathrm{H}=$ South Fork Harrods creek watershed. Urban subcatchments contain $\geq 30 \%$ impervious surface cover, rural subcatchments contain $\leq 10 \%$ impervious surface cover, and suburban subcatchments contain values in between 10 and $30 \%$ impervious surface cover. 


\section{CHAPTER 5: CONCLUSIONS}

Woody plant and groundcover (herbs and woody vines) assemblages in riparian areas in Metro Louisville were both affected by land use (urban, suburban, rural) measured using degree of impervious surface cover at catchment and smaller scales. Urban riparian plant assemblages were dominated by exotic species more so than those alongside rural streams where forests were a more dominant land-cover type. The exotic, invasive shrub L. maackii was the most prevalent woody species, and the most abundant species in urban woody plant communities. E. fortunei, an exotic woody vine, dominated the herb and woody vine plant communities in urban riparian areas. Not surprisingly, species densities of $L$. maackii and \% cover of $E$. fortunei were highly correlated $\left(\mathrm{r}^{2}=\right.$ 0.66). Conversely, native species were more likely found in riparian assemblages surrounded by forest cover at the catchment scale. The most common native woody species in forested riparian areas were $A$. triloba (understory tree) and L. benzoin (shrub). Wetland species (woody and herbaceous groundcover species) also demonstrated a greater affinity for forested riparian sites than for urban ones. As these species are more commonly associated with wetter environmental conditions, a sustained moisture gradient is implied along this urban-to-rural gradient of riparian sites, with urban being drier and rural sites being lower. This pattern of drier soils is expected and was found in 2008 when soils in urban sites were found to be drier in summer and fall than in suburban and rural sites.

However, surprisingly, given these wetland species associations with rural site 
groupings, streambank height adjacent to the sites displayed no obvious associations with site assemblage composition even though the highest streambanks (and presumably less flooded and drier soils) were found in urban areas. This demonstrates that other factors, including composition of the regional species pool and arrival at sites, and human riparian management and other anthropogenic factors also affect riparian plant composition. Variation in species diversity indices with land use was greater for groundcover herbs and woody vines as opposed to woody tree and shrub species. I found that riparian groundcover species diversity decreased with increasing percent impervious surface coverage within a $1-\mathrm{km}$ site radius. Groundcover species diversity also decreased with increasing lateral distance from the stream, and increased with increasing upstream catchment area in suburban riparian zones. The presence of exotic invasive species was also found to contributesto the reduction in plant species diversity for the groundcover community in more urban sites.

Since soil water levels differed among urban, suburban, and rural riparian zones, I expected soil gas fluxes of three important greenhouse gases $\left(\mathrm{CO}_{2}, \mathrm{CH}_{4}\right.$ and $\left.\mathrm{N}_{2} \mathrm{O}\right)$ to differ with land use as well. Carbon dioxide $\left(\mathrm{CO}_{2}\right)$ flux rates were related to proportion of impervious surface within a $1-\mathrm{km}$ proximity, but the strength of this relationship varied by season. Carbon dioxide flux rates increased with increasing soil temperatures across all sites (Fig. 4-1b \& 4-6), but site differences in soil temperatures played the greatest role in explaining differences in $\mathrm{CO}_{2}$ release during the hottest months and into fall, which is also when the effects of land-use manifested itself more clearly. Greater proportions of impervious surface within a $1-\mathrm{km}$ radius and higher soil temperatures resulted in greater $\mathrm{CO}_{2}$ release from the soil (Tables 4-5 \& 4-6). 
Methane and nitrous oxide flux patterns were more complex to explain, since net flux of each is determined by both aerobic and anaerobic processes. Differences in methane flux among sites in relation to their soil and site differences were more obvious in summer and fall. Fall net methane uptake rates were negatively correlated with $\mathrm{C}: \mathrm{N}$ ratios (Fig. 4-18), which may be attributable to greater methane-oxidizing nitrifier activity (Jones and Morita 1983, Bedard and Knowles 1989). Soil moisture levels also affect microbial activity by affecting the oxygenation levels of soil pores. Across these riparian sites, higher soil moisture values $(>\sim 0.35-\mathrm{g}$ soil water/g dry soil) were correlated with a shift from net methanotrophy (net methane uptake by soils) to net methanogenesis (net methane release from soil), because anaerobic, wet soils likely increased methanogen activity. Methane uptake, however, did not seem to be affected in a linear fashion by the range of soil moisture levels below $\sim 0.35$-g soil water/g dry soil (Fig. 4-17 \& 4-18b). Nitrous oxide efflux rates showed a strong seasonal, and variable, response between hot, dry August and cooler October (Fig. 4-19). No measured environmental site differences could explain the $\mathrm{N}_{2} \mathrm{O}$ flux variation observed among sites. However, release of $\mathrm{N}_{2} \mathrm{O}$ is the product or by-product of two different soil processes: anaerobic denitrification and aerobic nitrification. The variable response of $\mathrm{N}_{2} \mathrm{O}$ release from the soil is likely due to the synergism between these two processes.

The greenhouse gas response in urban, suburban, and rural riparian zones is varable (Fig. 4-6, 4-11, \& 4-19). While urban riparian soils exhibited some of the highest $\mathrm{CO}_{2}$ fluxes from the soil during summer, some of that debt was paid back by way of net $\mathrm{CH}_{4}$ uptake. Conversely, rural riparian soils exhibited lower $\mathrm{CO} 2$ flux rates; however, net methane emissions into the atmosphere occurred throughout spring and early summer. 
Carbon dioxide flux from suburban riparian soils was also relatively lower during summer, but forested suburban riparian zones were the least wide among the three landuse types, effectively reducing the carbon-savings. These findings complicate the determination of greenhouse gas budgets; however, considerations based on land-use are essential for accurate determination of climate alterations and formulating the appropriate response. 


\section{REFERENCES}

Ambus, P. and S. Christensen (1995). "Atmospheric pollutants and trace gases." Journal of Environmental Quality 24: 993-1001.

Arnold, C. L. and C. J. Gibbons (1996). "Impervious surface coverage: the emergence of a key environmental indicator." Journal of the the American Planning Association 62(2): 243-258.

ASTM Standard D422-63 (2002). Standard Test Method for Particle-Size Analysis of Soils. West Conshohocken, PA, ASTM International: 1-8.

Auble, G. T., J. M. Friedman, et al. (1994). "Relating riparian vegetation to present and future streamflows." Ecological Applications 4: 544-554.

Ball, B. C. (1999). "Field $\mathrm{N}_{2} \mathrm{O}, \mathrm{CO}_{2}$, and $\mathrm{CH}_{4}$ fluxes in relation to tillage, compaction and soil quality in Scotland." Soil and Tillage Research 53: 29-39.

Bedard, C. and R. Knowles (1989). "Physiology, biochemistry and specific inhibitors of $\mathrm{CH} 4, \mathrm{NH} 4$, and $\mathrm{CO}$ oxidation by methanotrophs and nitrifiers." Microbiological Reviews 53: $68-84$.

Belnap, J., C. V. Hawkes, et al. (2003). "Boundaries in miniature: two examples from soil." Bioscience 53(8): 739-749.

Bendix, J. (1997). "Flood disturbance and the distribution of riparian species diversity." The Geographical Review 87: 468-483.

Bendix, J. and C. R. Hupp (2000). "Hydrological and geomorphological impacts on riparian plant communities." Hydrological Processes 14: 2977-2990.

Boothroyd, I. K. G., J. M. Quinn, et al. (2004). "Riparian buffers mitigate effects of pine plantation logging on New Zealand streams 1. riparian vegetation structure, stream geomorphology and periphyton." Forest Ecology and Management 194: 199-213.

Borgmann, K. L. and A. D. Rodewald (2005). "Forest restoration in urbanizing landscapes: interactions between land uses and exotic shrubs." Restoration Ecology 13(2): $334-340$. 
Bray, H. R. and L. T. Kurtz (1945). "Determination of total, organic, and available forms of phosphorus in soils." Soil Science 59: 39-45.

Bren, L. J. (1992). "Tree invasion of an intermittent wetland in relation to changes in the flooding frequency of the River Murray." Australian Journal of Ecology 17: 395-408.

Brenner, F. J., J. J. Mondok, et al. (1991). "Impact of riparian areas and land use on four non-point source pollution parameters in Pennsylvania." Journal of the Pennsylvania Academy of Science 65(2): 65-70.

Brown, R. L. and R. K. Peet (2003). "Diversity and invasibility of southern Appalachian plant communities." Ecology 84(1): 32-39.

Burns, R. M. and B. H. Honkala (1990). Hardwoods. Agriculture Handbook 654. F. S. U. S. Department of Agriculture. Washington, DC. 2: 877.

Burton, M. L. and L. J. Samuelson (2008). "Influence of urbanization on riparian forest diversity and structure in the Georgia Piedmont, US." Plant Ecology 195: 99-115.

Burton, M. L., L. J. Samuelson, et al. (2005). "Riparian woody plant diversity and forest structure along an urban-rural gradient." Urban Ecosystems 8: 93-106.

Cadenasso, M. L. and S. T. A. Pickett (2001). "Effect of edge structure on the flux of species into forest interiors." Conservation Biology 15(1): 91-97.

Castellano, S. M. and R. L. Boyce (2007). "Spatial patterns of Juniperus virginiana and Lonicera maackii on a road cut in Kentucky, USA." Journal of the Torrey Botanical Society 134(2): 188-198.

Castro, M. S., P. A. Steudler, et al. (1995). "Factors controlling atmospheric methane consumption in temperate forest soils." Global Biogeochemical Cycles 9: 1-10.

Collier, M. H., J. L. Vankat, et al. (2002). "Diminished plant richness and abundance below Lonicera maackii, an invasive shrub." American Midland Naturalist 147: 60-71.

Colwell, R. K. (2009). EstimateS: Statistical estimation of species richness and shared species from samples.

Conner, E. F. and E. D. McCoy (1979). "The statistics and biology of the species-area relationship." The American Naturalist 113(6): 791-833.

Cooper, J. R., J. W. Gilliam, et al. (1987). "Riparian areas as filters for agricultural sediment." Soil Science Society of America Journal 51(2): 416-420. 
Cordell, H. K. and E. A. Macie, Eds. (2002). Population and Demographic Trends. Human Influences on Forest Ecosystems: The Southern Wildland-Urban Interface Assessment, U.S. Dept. of Agriculture. Forest Service.

Crill, P. M. (1991). "Seasonal patterns of methane uptake and carbon dioxide release by temperate woodland soil." Global Biogeochemical Cycles 5: 319-334.

Davidson, E. A., E. Belk, et al. (1998). "Soil water content and temperature as independent or confounded factors controlling soil respiration in a temperate mixed hardwood forest." Global Change Biology 4(2): 217-227.

Davidson, E. A., P. A. Matson, et al. (1993). "Processes regulating soil emissions of NO and $\mathrm{N}_{2} \mathrm{O}$ in a seasonally dry tropical forest." Ecology 74(1): 130-139.

Dorr, H., L. Katruff, et al. (1993). "Soil texture parameterization of the methane uptake in aerated soils." Chemosphere 26: 697-713.

Draaijers, G. P. J., W. P. M. Ivens, et al. (1988). "Atmospheric deposition in forest edges measured by monitoring canopy throughfall." Water, Air, and Soil Pollution 42: 129-136.

Dufrene, M. and P. Legendre (1997). "Species assemblages and indicator species: the need for a flexible asymmetrical approach." Ecological Monographs 67: 345-366.

Dunne, T. and L. B. Leopold (1978). Water in Environmental Planning. San Francisco, Freeman.

Easson, G. and L. D. Yarbrough (2002). "The effects of riparian vegetation on bank stability." Environmental \& Engineering Geoscience 8(4): 247-260.

Edwards, N. T. (1975). "Effects of Temperature and Moisture on Carbon Dioxide Evolution in a Mixed Deciduous Forest Floor." Soil Science Society of America Journal 39: 361-365.

Ehrenfeld, J. G. (2003). "Effects of exotic plant invasions on soil nutrient cycling processes." Ecosystems 6: 503-523.

ESRI (2010). ArcGIS. Redlands, CA.

Forman, R. T. T. (1995). Land mosaics: the ecology of landscapes and regions. New York, Cambridge University Press.

Fraver, S. (1994). "Vegetation responses along edge-to-interior gradients in the mixed hardwood forests of the Roanoke River basin, North Carolina." Conservation Biology 8(2): 822-832. 
Gehlhausen, S. M., M. W. Schwartz, et al. (2000). "Vegetation and microclimatic edge effects in two mixed-mesophytic forest fragments." Plant Ecology 147: 21-35.

Geyer, W., K. Brooks, et al. (2003). "Streambank stability of two Kansas river systems during the 1993 flood in Kansas, USA." Transactions of the Kansas Academy of Science 106(1/2): 48-53.

Gilliam, J. W. (1994). "Riparian wetlands and water quality." Journal of Environmental Quality 23: 896-900.

Gregory, S. V., F. J. Swanson, et al. (1991). "An ecosystem perspective of riparian zones: focus on links between land and water." Bioscience 41(8): 540-551.

Groffman, P. M., D. J. Bain, et al. (2003). "Down by the riverside: urban riparian ecology." Frontiers in Ecology and Environment 1(6): 315-321.

Groffman, P. M., N. J. Boulware, et al. (2002). "Soil Nitrogen Cycle Processes in Urban Riparian Zones." Environ. Sci. Technol. 36(21): 4547-4552.

Groffman, P. M., N. L. Law, et al. (2004). "Nitrogen fluxes and retention in urban watershed ecosystems." Ecosystems 7: 393-403.

Groffman, P. M., R. V. Pouyat, et al. (2006). "Land use context and natural soil controls on plant community composition and soil nitrogen and carbon dynamics in urban and rural forests." Forest Ecology and Management 236: 177-192.

Guillaume, D. (2002). "Patterns of plant species and community diversity at different organization levels in a forested riparian landscape." Journal of Vegetation Science 13: 91-106.

Hach, I. (2004). Water Analysis Handbook: Method 8039. Water Analysis Handbook, Hach Company.

Hach, I. (2004). Water Analysis Handbook: Method 8192. Water Analysis Handbook, Hach Company.

Homer, C., C. Huang, et al. (2004). "Development of a 2001 national landcover database for the United States." Photogrammetric Engineering and Remote Sensing 70(7): 829840 .

Hupp, C. R. (1982). "Stream-grade variation and riparian-forest ecology along Passage Creek, Virginia." Bulletin of the Torrey Botanical Club 109(4): 488-499.

Hutchinson, T. F. and J. L. Vankat (1997). "Invasibility and effects of Amur honeysuckle in southwestern Ohio forests." Conservation Biology 11: 1117-1124. 
Jacobs, T. J. and J. W. Gilliam (1985a). "Riparian losses of nitrate from agricultural drainage waters." Journal of Environmental Quality 14: 472-478.

Johnson, W. C., Ed. (1993). Divergent responses of riparian vegetation to flow regulation on teh Missouri and Platted rivers. Proceedings Symposium on the Restoration Planning for the Rivers of the Mississippi River Ecosystem. Washington D. C., U. S. Department of the Interior.

Jones, J. B. and P. J. Mulholland (1998). "Influence of drainage basin topography and elevation on carbon dioxide and methane supersaturation of stream water."

Biogeochemistry 40(1): 57-72.

Jones, R. D. and R. Y. Morita (1983). "Methane oxidation by Nitrosococcus oceanus and Nitrosomonas europaea." Applied Environmental Microbiology 45(2): 401-410.

Jones, R. L. (2005). Plant life of Kentucky: an illustrated guide to the vascular flora. Lexington, University Press of Kentucky.

Kalliola, R. and M. Puhakka (1988). "River dynamics and vegetation mosaicism: a case study of the river Kamajohka, northernmost Finland." Journal of Biogeography 15(5/6): 703-719.

Kendle, T. and S. Forbes (1997). Urban nature conservation: landscape management in the urban countryside. London, E \& FN Spon.

Kentucky Environmental and Public Protection Cabinet (2008). Final 2008 Integrated Report to Congress on the Condition of Water Resources in Kentucky. Division of Water. 2: 605 .

Kiffney, P. M., C. J. Volk, et al. (2004). "A high-severity disturbance event alters community and ecosystem properties in West Twin creek, Olympic National Park, Washington, USA." American Midland Naturalist 152(1): 286-303.

Kinley, T. A. and N. J. Newhouse (1997). "Relationship of riparian reserve zone width to bird density and diversity in southeastern British Columbia." Northwest Science 71(2): $75-86$.

Kolenbrander, G. J. (1972). "The eutrophication of surface water by agriculture and the urban population." Dutch Nitrogenous Fertilizer Review 15: 56-57.

Lemmon, P. E. (1956). "A spherical densiometer for estimating forest overstory density." Forest Science 2(4): 314-320.

Lemmon, P. E. (1957). "A new instrument for measuring forest overstory density." Journal of Forestry 55(9): 667-668. 
Linsay, W. L. and W. A. Norvell (1978). "Development of a DTPA soil test for zinc, iron, manganese, and copper." Soil Science Society of America Journal 42: 421-428.

Lite, S. J., K. J. Bagstad, et al. (2005). "Riparian plant species richness along lateral and longitudinal gradients of water stress and flood disturbance, San Pedro River, Arizona, USA." Journal of Arid Environments 63(4): 785-813.

Loewenstein, N. J. and E. F. Loewenstein (2005). "Non-native plants in the understory of riparian forests across a land use gradient in the Southeast." Urban ecosystems 8: 79-91.

\section{LOJIC (2010). Louisville/Jefferson County Information Consortium.}

Lowrance, R., L. S. Altier, et al. (1997). "Water quality functions of riparian forest buffers in Chesapeake Bay watersheds." Environmental Management 21(5): 687-712.

Lowrance, R. R., S. McIntyre, et al. (1988). "Erosion and deposition in a field/forest system estimated using cesium-137 activity." Journal of Water Conservation 43: 195 199.

Luken, J. O. and J. W. Thieret (1996). "Amur honeysuckle, its fall from grace." Bioscience 46(1): 18-24.

MacDougall, A. S. and M. Kellman (1992). "The understorey light regime and patterns of tree seedlings in tropical riparian forest patches." Journal of Biogeography 19: 667-675.

Magurran, A. E. (2004). Measuring Biological Diversity, Blackwell.

McCune, B. and J. B. Grace (2002). Analysis of Ecological Communities. Glenedon Beach, OR, MjM Software Design.

McCune, B. and M. J. Mefford (1999). PC-Ord. Multivariate Analysis of Ecological Data. Gleneden Beach, Oregon, U.S.A., MjM Software.

McKinney, M. L. (2004). "Citizens as propagules for exotic plants: measurement and management implications." Weed Technology 18: 1480-1483.

McKinney, M. L. (2008). "Effects of urbanization on species richness: a review of plants and animals." Urban Ecosystems 11(2): 161-176.

McLean, E. O., Ed. (1982). Soil pH and lime requirement. Methods of Soil Analysis. Part 2. Chemical and Microbiological Properties. Madison, WI, ASA Monograph No. 9.

Mehlich, A. (1984). "Mehlich-3 soil test extractant: A modification of Mehlich-2 extractant." Communications in soil science and plant analysis 15: 1409-1416. 
Merriam, R. W. and E. Feil (2002). "The potential impact of an introduced shrub and on native plant diversity and forest regeneration." Biological Invasions 4: 369-373.

Metropolitan Sewer District (1999). "Water Quality: Goose Creek Watershed." Retrieved Jan. 5, 2010, from http://www.msdlouky.org/insidemsd/goosecreek.htm.

Metropolitan Sewer District (1999). "Water Quality: Middle Fork of Beargrass Creek Watershed." from hitp:/www.msdlouky.org/insidemsd/middlebeargrass.htm.

Metropolitan Sewer District (1999). WATERS of Jefferson County: Year Zero. Louisville, KY: 402.

Meyer, J. L., M. J. Paul, et al. (2005). "Stream ecosystem function in urbanizing landscapes." Journal of the North American Benthological Society 24(3): 602-612.

Moffatt, S. F. and S. M. McLachlan (2004). "Understorey indicators of disturbace for riparian forests along an urban-rural gradient in Manitoba." Ecological Indicators 4: 1-16.

Moore, A. A. and M. A. Palmer (2005). "Invertebrate biodiversity in agricultural and urban headwater streams: implications for conservation and management." Ecological Applications 15(4): 1169-1177.

Naiman, R. J. and H. Decamps (1997). "The ecology of interfaces: riparian zones." Annual Review of Ecological Systems 28: 621-658.

National Research Council (2002). Riparian Areas: Functions and Strategies for Management. Washington, D. C., National Academy Press.

Neller, R. J. (1989). "Induced channel enlargement insmall urban catchments, Armidale, New South Wales." Environmental Geology and Water Sciences 14: 167-172.

Nilsson, C. (1981). "Riparian vegetation of northern Swedish rivers." Wahlenbergia 7: 113-124.

Nilsson, C., A. Ekblad, et al. (1994). "A comparison of species richness and traits of riparian plants between a main river channel and its tributaries." Journal of Ecology 82: 281-295.

Nilsson, C., A. Ekblad, et al. (1991). "Long term effects of river regulation on river margin vegetation." The Journal of Applied Ecology 28(3): 963-987.

Nilsson, C., R. Jansson, et al. (1997). "Long-term responses of river-margin vegetation to water-level regulation." Science 276: 798-800.

Nyland, R. D. and K. E. Remele (1973). Research Note \#17: Prism measures distance for plot boundaries., SUNY-Syracuse Applied Forestry Research Institute: 1-4. 
Parendes, L. A. and J. A. Jones (2000). "Role of light availability and dispersal in exotic plant invasion along roads and streams in the H. J. Andrews Experimental Forest, Oregon." Conservation Biology 14(1): 64-75.

Paul, M. J. and J. L. Meyer (2001). "Streams in the urban landscape." Annual Review of Ecological Systems 32: 333-365.

Pollock, M. M., R. J. Naiman, et al. (1998). "Plant species richness in riparian wetlands a test of biodiversity theory." Ecology 79(1): 94-105.

Porter, E. E., B. R. Forschner, et al. (2001). "Woody vegetation and canopy fragmentation along a forest-to-urban gradient." Urban Ecosystems 5: 131-151.

Puckett, L. J. (2004). "Hydrogeologic controls on the transport and fate of nitrate in ground water beneath riparian buffer zones: results from thirteen studies across the United States." Water Science and Technology 49(3): 47-53.

R Development Core Team (2009). R: A Language and Environment for Statistical Computing. Vienna, Austria, R Foundation for Statistical Computing.

Raich, J. W. and W. H. Schlesinger (1992). "The global carbon dioxide flux in soil respiration and its relationship to vegetation and climate." Tellus 44B: 81-99.

Robertson, P. A., G. T. Weaver, et al. (1978). "Vegetation and tree species patterns near the northern terminus of the southern floodplain forest." Ecological Monographs 48: 249267.

Ross, D., Ed. (1995). Recommended soil tests for determining exchange capacity. Recommended soil testing procedures for the northeastern United States. Northeastern Regional Bulletin \#493. Newark, DE, Ag Experiment Station, University of Delaware.

Rudolph, D. C. and J. G. Dickson (1990). "Streamside zone width and amphibian and reptile abundance." The Southwestern Naturalist 35(4): 472-476.

Rustad, L. E., T. G. Huntington, et al. (2000). "Controls on soil respiration: Implications for climate change." Biogeochemistry 48(1): 1-6.

Samuelson, L. J. and M. E. Hogan (2006). Forest trees: a guide to the eastern United States. Upper Saddle River, NJ, Pearson Education, Inc.

Schimel, J. P., E. A. Holland, et al., Eds. (1993). Controls on methane flux from terrestrial systems. Agroecosystem effects on radiatively active trace gasses and global climate change. Madison, WI, American Society of Agronomy.

Schueler, T. R. (1994). "The importance of imperviousness." Watershed Protection Techniques 1: 100-111. 
Schulte, E. E. and B. G. Hopkins, Eds. (1996). Estimation of soil organic matter by weight loss-on-ignition. Soil organic matter: Analysis and interpretation. Madison, WI, Soil Science Society of America, No. 46.

Shannon, C. E. (1948). "A mathematical theory of communication." Bell System Technical Journal 27: 379-423, 623-656.

Simpson, E. H. (1949). "Measurement of diversity." Nature 163: 688.

Stander, E. K. and J. G. Ehrenfeld (2010). Urban Riparian Function. Urban Ecosystem Ecology. J. Aitkenhead-Peterson and A. Volder. Madison, WI, American Society of Agronomy, Crop Science Society of America, Soil Science Society of America: 253-274.

Steudler, P. A., R. D. Bowden, et al. (1989). "Influence of nitrogen fertilization on methane uptake in temperate forest soils." Nature 341: 314-316.

Tickner, D. P., P. G. Angold, et al. (2001). Hydrology as an influence on invasion. Plant Invasions: Species Ecology and Ecosystem Management. G. Brundu, J. Brock, I. Camarda, L. Child and M. Wade. Netherlands, Backhuys Publishers: 159-167.

Torn, M. S. and J. Harte (1996). "Methane consumption by montane soils: Implications for positive and negative feedback with climatic change." Biogeochemistry 32: 53-67.

Trammell, T. L. E. (2010). The forgotten forest: ecosystem structure, function, and services of forested verges along interstates in Louisville, KY. Biology. Louisville, KY, University of Louisville. PhD.: 227.

U. S. Department of Agriculture and Natural Resources Conservation Service (2009).

"Native, Invasive, and Other Plant Related Definitions." from http: www.et.nres.usda.gov/plant definitions.html.

U. S. Department of Agriculture and Natural Resources Conservation Service (2010). The PLANTS Database, National Plant Data Center, Baton Rouge, La 70874-4490 USA.

U. S. Department of the Interior and U. S. Geological Survey (2008, November 4, 2008). "NLCD 2001 Land Cover Class Definitions." from http:/www.mrlc.gov/nled definitions.php.

Vidra, R. L., T. H. Shear, et al. (2006). "Testing the paradigms of exotic species invasion in urban riparian forests." Natural Areas Journal 26(4): 339-350.

Walsh, C. J. (2000). "Urban impacts on the ecology of receiving waters: a framework for assessment, conservation and restoration." Hydrobiologia 431: 107-114. 
Walsh, C. J., A. H. Roy, et al. (2005). "The urban stream syndrome: current knowledge and the search for a cure." Journal of the North American Benthological Society 24(3): 706-723.

Wenger, S. (1999). A review of the scientific literature on riparian buffer width, extent and vegetation. Athens, University of Georgia: 1-59.

Whalen, S. C., W. S. Reeburgh, et al. (1990). "Rapid methane oxidation in a landfill cover soil." Applied Environmental Microbiology 56: 3405-3411.

Wharton, M. E. and R. W. Barbour (1973). Trees and Shrubs of Kentucky. Lexington, University Press of Kentucky.

Wright, C. J. and D. C. Coleman (2002). "Responses of soil microbial biomass, nematode trophic groups, $\mathrm{N}$-mineralization and litter decomposition to disturbance events in teh southern Appalachians." Soil Biological Chemistry 34: 13-25.

$\mathrm{Xu}, \mathrm{M}$. and Y. Qi (2001). "Soil-surface $\mathrm{CO}_{2}$ efflux and its spatial and temporal variations in a young ponderosa pine plantation in northern California." Global Change Biology 7(6): $667-668$.

Yeakley, J. A., D. C. Coleman, et al. (2003). "Hillslope nutrient dynamics following upland riparian vegetation disturbance." Ecosystems 6: 154-167.

Zipperer, W. C., Ed. (2002). Urban Influences on Forests. Human Influences on Forest Ecosystems: The Southern Wildland-Urban Interface Assessment, U. S. Department of Agriculture. Forest Service.

Zweifel, U. L., J. Wikner, et al. (1995). "Dynamics of dissolved organic carbon in a coastal ecosystem." Limnology and Oceanography 40(2): 299-305. 
Appendix Table 1: Tree species within 41 riparian sites in Jefferson and Oldham counties in Kentucky. Site names refer to the watershed in which the site was located, where $\mathrm{B}=$ Middle Fork Beargrass, $\mathrm{G}=$ Goose, and $\mathrm{H}=$ South Fork Harrods Creek watersheds. Relative importance values (IV) were calculated from relative density and basal area by land cover category. Land use categories for each site were determined by the proportion of impervious surface (IS) within the subcatchment in which the site was located. Urban $\geq 30 \%$ IS, Rural $\leq 10 \%$ IS, and Suburban is between 10 and $30 \%$ IS.

\begin{tabular}{|c|c|c|c|c|c|}
\hline Site & $\begin{array}{l}\text { Land use } \\
\text { category }\end{array}$ & Tree Species Names & $\begin{array}{l}\text { Density } \\
\text { (stems/ } \\
\text { ha) }\end{array}$ & $\begin{array}{l}\text { Basal } \\
\text { area } \\
\left(\mathbf{m}^{2} / \mathbf{h a}\right)\end{array}$ & IV \\
\hline $\mathrm{G} 2$ & Rural & Acer saccharum Marsh. & 267 & 18.1 & 86 \\
\hline $\mathrm{G} 2$ & Rural & Fraxinus americana $\mathrm{L}$. & 67 & 0.0 & 9 \\
\hline $\mathrm{G} 2$ & Rural & Maclura pomifera (Raf.) Schneid. & 400 & 18.4 & 105 \\
\hline G7 & Rural & Acer negundo L. & 200 & 1.1 & 200 \\
\hline $\mathrm{H} 1$ & Rural & Acer negundo L. & 200 & 10.7 & 48 \\
\hline $\mathrm{H} 1$ & Rural & Asimina triloba (L.) Dunal & 100 & 0.2 & 13 \\
\hline $\mathrm{H} 1$ & Rural & Cercis canadensis L. & 200 & 1.5 & 28 \\
\hline $\mathrm{H} 1$ & Rural & Juglans nigra L. & 200 & 34.6 & 98 \\
\hline $\mathrm{H} 1$ & Rural & Ulmus rubra Muhl. & 100 & 0.3 & 13 \\
\hline $\mathrm{H} 11$ & Rural & Acer negundo L. & 100 & 4.9 & 30 \\
\hline $\mathrm{H} 11$ & Rural & Fraxinus americana $\mathrm{L}$. & 600 & 9.1 & 108 \\
\hline $\mathrm{H} 11$ & Rural & Platanus occidentalis L. & 100 & 13.5 & 62 \\
\hline $\mathrm{H} 12$ & Rural & Acer negundo L. & 50 & 0.1 & 26 \\
\hline $\mathrm{H} 12$ & Rural & Fraxinus americana $\mathrm{L}$. & 50 & 0.4 & 31 \\
\hline $\mathrm{H} 12$ & Rural & Juglans nigra L. & 50 & 5.4 & 115 \\
\hline $\mathrm{H} 12$ & Rural & Prunus serotina Ehrh. & 50 & 0.2 & 28 \\
\hline $\mathrm{H} 14$ & Rural & Acer negundo L. & 350 & 3.4 & 111 \\
\hline $\mathrm{H} 14$ & Rural & Juglans nigra L. & 50 & 10.9 & 89 \\
\hline H5 & Rural & Aesculus glabra Willd. & 100 & 1.0 & 44 \\
\hline H5 & Rural & Asimina triloba (L.) Dunal & 100 & 0.1 & 23 \\
\hline H5 & Rural & Celtis occidentalis $\mathrm{L}$. & 100 & 0.3 & 27 \\
\hline H5 & Rural & Maclura pomifera (Raf.) Schneid. & 200 & 2.7 & 106 \\
\hline H6 & Rural & Acer saccharum Marsh. & 277 & 2.2 & 161 \\
\hline H6 & Rural & Cornus florida $\mathrm{L}$. & 139 & 0.1 & 39 \\
\hline $\mathrm{H} 7$ & Rural & Asimina triloba (L.) Dunal & 1211 & 1.6 & 153 \\
\hline $\mathrm{H} 7$ & Rural & Carpinus caroliniana Walter & 173 & 0.4 & 29 \\
\hline $\mathrm{H} 7$ & Rural & Cornus florida L. & 173 & 0.1 & 18 \\
\hline $\mathrm{H} 8$ & Rural & Acer negundo L. & 50 & 1.3 & 13 \\
\hline $\mathrm{H} 8$ & Rural & Acer saccharum Marsh. & 50 & 1.5 & 14 \\
\hline
\end{tabular}




\begin{tabular}{|c|c|c|c|c|c|}
\hline $\mathrm{H} 8$ & Rural & $\begin{array}{l}\text { Appendix Table } 1 \text { (cont.) } \\
\text { Aesculus glabra Willd. }\end{array}$ & 300 & 0.6 & 39 \\
\hline $\mathrm{H} 8$ & Rural & Celtis occidentalis L. & 100 & 1.8 & 22 \\
\hline H8 & Rural & Juglans nigra L. & 50 & 8.9 & 57 \\
\hline H8 & Rural & Quercus alba L. & 50 & 2.8 & 22 \\
\hline H8 & Rural & Ulmus rubra Muhl. & 250 & 0.7 & 33 \\
\hline H9 & Rural & Juniperus virginiana L. & 1000 & 38.6 & 200 \\
\hline B11 & Suburban & Celtis occidentalis L. & 358 & 0.2 & 63 \\
\hline B11 & Suburban & Staphylea trifolia L. & 717 & 0.5 & 137 \\
\hline $\mathrm{B} 12$ & Suburban & Acer negundo L. & 400 & 8.4 & 88 \\
\hline B12 & Suburban & Tilia americana $\mathrm{L}$. & 500 & 10.7 & 112 \\
\hline G1 & Suburban & Celtis occidentalis L. & 100 & 0.1 & 22 \\
\hline G1 & Suburban & Juglans nigra L. & 100 & 1.7 & 89 \\
\hline Gl & Suburban & Platanus occidentalis L. & 100 & 0.4 & 36 \\
\hline G1 & Suburban & Ulmus rubra Muhl. & 200 & 0.3 & 52 \\
\hline G10 & Suburban & Acer negundo L. & 400 & 23.2 & 200 \\
\hline G11 & Suburban & Acer negundo L. & 600 & 2.9 & 105 \\
\hline G11 & Suburban & Celtis occidentalis L. & 100 & 0.9 & 24 \\
\hline G11 & Suburban & Fraxinus americana $\mathrm{L}$. & 300 & 1.9 & 60 \\
\hline G11 & Suburban & Juglans nigra L. & 100 & 0.1 & 10 \\
\hline G12 & Suburban & Acer saccharum Marsh. & 300 & 0.6 & 53 \\
\hline G12 & Suburban & Fraxinus americana $\mathrm{L}$. & 300 & 19.3 & 147 \\
\hline G13 & Suburban & Acer negundo L. & 100 & 5.6 & 130 \\
\hline G13 & Suburban & Ulmus rubra Muhl. & 100 & 1.4 & 70 \\
\hline G3 & Suburban & Acer saccharinum L. & 400 & 12.0 & 54 \\
\hline G3 & Suburban & Morus alba $\mathrm{L}$. & 300 & 8.0 & 38 \\
\hline G3 & Suburban & Ulmus rubra Muhl. & 1000 & 19.0 & 108 \\
\hline G6 & Suburban & Acer negundo L. & 100 & 2.8 & 81 \\
\hline G6 & Suburban & Aesculus glabra Willd. & 100 & 0.2 & 25 \\
\hline G6 & Suburban & Asimina triloba (L.) Dunal & 200 & 0.7 & 56 \\
\hline G6 & Suburban & Ulmus rubra Muhl. & 100 & 0.8 & 38 \\
\hline G8 & Suburban & Acer negundo L. & 145 & 0.8 & 23 \\
\hline G8 & Suburban & Acer saccharum Marsh. & 291 & 5.0 & 57 \\
\hline G9 & Suburban & Acer negundo $\mathrm{L}$. & 1000 & 9.1 & 200 \\
\hline H10 & Suburban & Acer saccharum Marsh. & 300 & 6.9 & 111 \\
\hline $\mathrm{H} 10$ & Suburban & Fraxinus americana $\mathrm{L}$. & 100 & 6.6 & 69 \\
\hline $\mathrm{H} 10$ & Suburban & Ulmus rubra Muhl. & 100 & 0.1 & 21 \\
\hline $\mathrm{H} 2$ & Suburban & Acer negundo L. & 200 & 13.0 & 81 \\
\hline $\mathrm{H} 2$ & Suburban & Acer saccharum Marsh. & 400 & 8.8 & 84 \\
\hline $\mathrm{H} 2$ & Suburban & Asimina triloba (L.) Dunal & 100 & 0.2 & 12 \\
\hline $\mathrm{H} 2$ & Suburban & Celtis occidentalis L. & 100 & 0.1 & 12 \\
\hline
\end{tabular}




\begin{tabular}{|c|c|c|c|c|c|}
\hline $\mathrm{H} 2$ & Suburban & $\begin{array}{l}\text { Appendix Table } 1 \text { (cont.) } \\
\text { Tilia americana L. }\end{array}$ & 100 & 0.1 & 11 \\
\hline $\mathrm{H} 4$ & Suburban & Celtis occidentalis L. & 263 & 0.2 & 24 \\
\hline $\mathrm{H} 4$ & Suburban & Cornus alternifolia L. f. & 526 & 2.8 & 114 \\
\hline $\mathrm{H} 4$ & Suburban & Fraxinus pennsylvanica Marsh. & 263 & 0.2 & 26 \\
\hline H4 & Suburban & Ulmus rubra Muhl. & 263 & 0.6 & 35 \\
\hline $\mathrm{B} 1$ & Urban & Acer negundo L. & 1000 & 40.6 & 190 \\
\hline B1 & Urban & Morus rubra L. & 50 & 0.1 & 5 \\
\hline $\mathrm{B} 1$ & Urban & Quercus rubra L. & 50 & 0.1 & 5 \\
\hline $\mathrm{B} 13$ & Urban & Morus alba L. & 654 & 11.8 & 200 \\
\hline B14 & Urban & Acer negundo L. & 400 & 8.7 & 58 \\
\hline B14 & Urban & Aesculus glabra Willd. & 100 & 6.7 & 21 \\
\hline B 14 & Urban & Fraxinus americana $\mathrm{L}$. & 200 & 10.2 & 38 \\
\hline B14 & Urban & Platanus occidentalis L. & 100 & 40.7 & 72 \\
\hline B14 & Urban & Ulmus rubra Muhl. & 100 & 0.2 & 11 \\
\hline $\mathrm{B} 2$ & Urban & Celtis occidentalis L. & 500 & 32.6 & 116 \\
\hline $\mathrm{B} 2$ & Urban & Platanus occidentalis L. & 100 & 67.9 & 84 \\
\hline B3 & Urban & Acer saccharum Marsh. & 400 & 0.7 & 33 \\
\hline B3 & Urban & $\begin{array}{l}\text { Carya cordiformis (Wangenh.) K. } \\
\text { Koch }\end{array}$ & 300 & 17.5 & 72 \\
\hline B3 & Urban & Celtis occidentalis L. & 500 & 10.8 & 68 \\
\hline B3 & Urban & Juglans nigra L. & 100 & 7.1 & 27 \\
\hline B4 & Urban & Acer negundo L. & 700 & 5.0 & 107 \\
\hline B4 & Urban & Acer saccharum Marsh. & 100 & 8.0 & 58 \\
\hline B4 & Urban & Morus rubra L. & 100 & 4.0 & 34 \\
\hline B5 & Urban & Acer negundo L. & 200 & 9.0 & 200 \\
\hline B6 & Urban & Fraxinus pennsylvanica Marsh. & 515 & 1.5 & 19 \\
\hline B6 & Urban & Morus alba L. & 1804 & 20.3 & 92 \\
\hline B6 & Urban & Platanus occidentalis L. & 258 & 19.6 & 41 \\
\hline B6 & Urban & Prunus cerasifera Ehrh. & 258 & 3.1 & 13 \\
\hline B6 & Urban & Ulmus rubra Muhl. & 258 & 15.9 & 35 \\
\hline B7 & Urban & Betula populifolia Marsh. & 33 & 0.0 & 6 \\
\hline B7 & Urban & Fraxinus americana $\mathrm{L}$. & 167 & 1.3 & 55 \\
\hline B7 & Urban & Juglans nigra L. & 133 & 3.0 & 83 \\
\hline B7 & Urban & Maclura pomifera (Raf.) Schneid. & 67 & 0.5 & 22 \\
\hline B7 & Urban & Thuja occidentalis $\mathrm{L}$. & 33 & 0.0 & 6 \\
\hline B7 & Urban & Ulmus rubra Muhl. & 133 & 0.2 & 27 \\
\hline B8 & Urban & Juglans nigra $\mathrm{L}$. & 200 & 11.5 & 200 \\
\hline B9 & Urban & Picea pungens Engelm. & 100 & 0.3 & 200 \\
\hline G8 & Urban & Celtis occidentalis $\mathrm{L}$. & 291 & 24.0 & 120 \\
\hline
\end{tabular}


Appendix Table 2: Wetland indicator status and species provenance codes used in this study and definitions (U. S. Department of Agriculture and Natural Resources Conservation Service 2009, 2010).

*Est. prob. - estimated probability.

\begin{tabular}{|c|c|c|}
\hline Code & Term & Meaning \\
\hline OBL & Obligate & $\begin{array}{l}\text { Occurs almost always (est. prob*. } 99 \% \text { ) } \\
\text { under natural conditions in wetlands }\end{array}$ \\
\hline FACW & $\begin{array}{l}\text { Facultative } \\
\text { wetland }\end{array}$ & $\begin{array}{l}\text { Usually occurs in wetlands (est. prob. } \\
67 \%-99 \% \text { ) }\end{array}$ \\
\hline FAC & Facultative & $\begin{array}{l}\text { Equally likely to occur in wetlands or } \\
\text { non-wetlands (est. prob. } 34 \%-66 \% \text { ) }\end{array}$ \\
\hline FACU & $\begin{array}{l}\text { Facultative } \\
\text { upland }\end{array}$ & $\begin{array}{l}\text { Usually occurs in non-wetlands (est. } \\
\text { prob. } 67 \%-99 \% \text { ) }\end{array}$ \\
\hline UPL & Upland & $\begin{array}{l}\text { Occurs in wetlands in another region, } \\
\text { but occurs almost always (est. prob. } \\
99 \% \text { ) in non-wetlands under natural } \\
\text { conditions }\end{array}$ \\
\hline $\mathrm{NI}$ & No indicator & $\begin{array}{l}\text { Insufficient information available to } \\
\text { determine an indicator status }\end{array}$ \\
\hline $\mathrm{N}$ & Native & $\begin{array}{l}\text { Plants that have developed over } \\
\text { hundreds of years in an area - pre- } \\
\text { European settlement }\end{array}$ \\
\hline $\mathrm{E}$ & Exotic & $\begin{array}{l}\text { Plants that have been introduced with } \\
\text { human assistance into an area in which } \\
\text { it was not found pre-European- } \\
\text { settlement }\end{array}$ \\
\hline Z & Naturalized & $\begin{array}{l}\text { Exotic species that do not need human } \\
\text { assistance to reproduce, thereby } \\
\text { maintaining themselves over an } \\
\text { unspecified period of time }\end{array}$ \\
\hline
\end{tabular}


Appendix Table 3: National Land Cover Database (U. S. Department of the Interior and U. S. Geological Survey 2008) land-cover classification categories, definitions, and examples. Table reproduced from U. S. Dept. of Interior and U.S. Geological Survey (2008)

\begin{tabular}{|c|c|}
\hline Classification & Definition \\
\hline Developed, Open Space & $\begin{array}{l}\text { Includes areas with a mixture of some constructed } \\
\text { structures, but mostly vegetation in the form of lawn } \\
\text { grasses. Impervious surfaces account for less than } 20 \% \\
\text { of total cover (e. g. park, golf course). }\end{array}$ \\
\hline Developed, Low Intensity & $\begin{array}{l}\text { Includes areas with a mixture of constructed structures } \\
\text { and vegetation. Impervious surfaces account for } 20- \\
49 \% \text { of total cover (e. g. single family housing). }\end{array}$ \\
\hline $\begin{array}{l}\text { Developed, Medium } \\
\text { Intensity }\end{array}$ & $\begin{array}{l}\text { Includes areas with a mixture of constructed structures } \\
\text { and vegetation. Impervious surfaces account for } 50- \\
79 \% \text { of the total cover (e. g. single family housing) }\end{array}$ \\
\hline Developed, High Intensity & $\begin{array}{l}\text { Includes highly developed areas where people reside or } \\
\text { work in high numbers. Impervious surfaces account for } \\
80-100 \text { percent of the total cover (Apartments, } \\
\text { Commercial/Industrial). }\end{array}$ \\
\hline Deciduous Forest & $\begin{array}{l}\text { Areas dominated by trees generally greater than } 5 \\
\text { meters tall, and greater than } 20 \% \text { of total vegetation } \\
\text { cover. More than } 75 \% \text { of the tree species shed foliage } \\
\text { simultaneously in response to seasonal change. }\end{array}$ \\
\hline Mixed Forest & $\begin{array}{l}\text { Areas dominated by trees generally greater than } 5 \\
\text { meters tall, and greater than } 20 \% \text { of total vegetation } \\
\text { cover. Neither deciduous nor evergreen species are } \\
\text { greater than } 75 \% \text { of total tree cover. }\end{array}$ \\
\hline Herbaceous & $\begin{array}{l}\text { Areas dominated by graminoid or herbaceous } \\
\text { vegetation, generally greater than } 80 \% \text { of total } \\
\text { vegetation. These areas are not subject to intensive } \\
\text { management such as tilling, but can be utilized for } \\
\text { grazing. }\end{array}$ \\
\hline Pasture & $\begin{array}{l}\text { Areas of grasses, legumes, or grass-legume mixtures } \\
\text { planted for livestock grazing or the production of seed } \\
\text { or hay crops, typically on a perennial cycle. Pasture/hay } \\
\text { vegetation accounts for greater than } 20 \% \text { of total } \\
\text { vegetation. }\end{array}$ \\
\hline Cultivated Crops & $\begin{array}{l}\text { Areas used for the production of annual and perennial } \\
\text { woody crops. Crop vegetation accounts for greater than } \\
20 \% \text { of total vegetation (e. g. corn, orchard) }\end{array}$ \\
\hline Wetlands & $\begin{array}{l}\text { Areas where soil or substrate is periodically saturated } \\
\text { with or covered with water. }\end{array}$ \\
\hline
\end{tabular}


Appendix Table 4: Tree, sapling, tree seedling, and shrub species within 41 riparian sites in Jefferson and Oldham counties in Kentucky. Sites were located in Middle Fork Beargrass, Goose, and South Fork Harrods Creek watersheds. Species codes and wetland indicator status were obtained from USDA PLANTS Database (2010).

Species sampled across all sites $(\mathrm{n}=41)$

\begin{tabular}{|c|c|c|}
\hline Trees & Code & $\begin{array}{l}\text { Wetland } \\
\text { Indicator } \\
\text { Status }\end{array}$ \\
\hline Acer negundo L. & ACNE2 & FAC \\
\hline Acer saccharinum L. & ACSA2 & FACW \\
\hline Acer saccharum Marsh. & $\mathrm{ACSA} 3$ & FACU \\
\hline Aesculus glabra Willd. & AEGL & FACU \\
\hline Asimina triloba (L.) Dunal & ASTR & FACU \\
\hline Betula populifolia Marsh. & BEPO & FAC \\
\hline Carpinus caroliniana Walter & CACA18 & FAC \\
\hline Carya cordiformis (Wangenh.) K. Koch & $\mathrm{CACO} 15$ & FACU \\
\hline Celtis occidentalis L. & CEOC & FACU \\
\hline Cercis canadensis L. & CECA4 & FACU \\
\hline Cornus alternifolia L. f. & COAL2 & NI \\
\hline Cornus florida $\mathrm{L}$. & COFL2 & FACU \\
\hline Fraxinus americana L. & FRAM2 & FACU \\
\hline Fraxinus pennsylvanica Marsh. & FRPE & FACW \\
\hline Juglans nigra L. & ЛNI & FACU \\
\hline Juniperus virginiana L. & JUVI & FACU \\
\hline Maclura pomifera (Raf.) Schneid. & MAPO & UPL \\
\hline Morus alba L.* & MOAL & UPL \\
\hline Morus rubra L. & MORU2 & FACU \\
\hline Picea pungens Engelm. & PIPU & $\mathrm{NI}$ \\
\hline Platanus occidentalis L. & PLOC & FACW \\
\hline Prunus cerasifera Ehrh.** & PRCE2 & $\mathrm{NI}$ \\
\hline Prunus serotina Ehrh. & PRSE2 & FACU \\
\hline Quercus alba L. & QUAL & FACU \\
\hline Quercus rubra L. & QURU & FACU \\
\hline Staphylea trifolia $\mathrm{L}$. & STTR & $\mathrm{FAC}$ \\
\hline Thuja occidentalis $\mathrm{L}$. & THOC2 & FACW \\
\hline Tilia americana L. & TIAM & FACU \\
\hline Ulmus rubra Muhl. & ULRU & FAC \\
\hline
\end{tabular}




\section{Appendix Table 4 (cont.)}

\begin{tabular}{|c|c|c|}
\hline Saplings & Code & $\begin{array}{l}\text { Wetland } \\
\text { Indicator } \\
\text { Status }\end{array}$ \\
\hline Acer negundo L. & ACNE2 & FAC \\
\hline Acer saccharum Marsh. & ACSA3 & FACU \\
\hline Aesculus glabra Willd. & AEGL & FACU \\
\hline Asimina triloba (L.) Dunal & ASTR & FACU \\
\hline Carya cordiformis (Wangenh.) K. Koch & $\mathrm{CACO} 15$ & FACU \\
\hline Celtis occidentalis L. & CEOC & FACU \\
\hline Cercis canadensis L. & $\mathrm{CECA} 4$ & FACU \\
\hline Cornus sericea L. ssp. sericea & COSES & FACW \\
\hline Cornus sp. & CORNU & \\
\hline Fraxinus americana $\mathrm{L}$. & FRAM2 & FACU \\
\hline Fraxinus pennsylvanica Marsh. & FRPE & $\mathrm{FACW}$ \\
\hline Fraxinus quadrangulata Michx. & FRQU & $\mathrm{NI}$ \\
\hline Juglans nigra L. & JUNI & FACU \\
\hline Maclura pomifera (Raf.) Schneid. & MAPO & UPL \\
\hline Morus rubra L. & MORU2 & FACU \\
\hline Prunus serotina Ehrh. & PRSE2 & FACU \\
\hline Quercus rubra L. & QURU & FACU \\
\hline Robinia pseudoacacia L. & ROPS & FAC \\
\hline Staphylea trifolia L. & STTR & FAC \\
\hline Thuja occidentalis & THOC2 & FACW \\
\hline Tilia americana $\mathrm{L}$. & TIAM & FACU \\
\hline Ulmus rubra Muhl. & ULRU & $\mathrm{FAC}$ \\
\hline Tree seedlings & Code & $\begin{array}{l}\text { Wetland } \\
\text { Indicator } \\
\text { Status }\end{array}$ \\
\hline Acer negundo L. & ACNE2 & FAC \\
\hline Acer saccharum Marsh. & ACSA3 & FACU \\
\hline Acer sp. & ACER & \\
\hline Aesculus glabra Willd. & AEGL & FACU \\
\hline Asimina triloba (L.) Dunal & ASTR & FACU \\
\hline Betula alleghaniensis Britt. & BEAL2 & FAC \\
\hline Carya cordiformis (Wangenh.) K. Koch & $\mathrm{CACO} 15$ & FACU \\
\hline Cercis canadensis $\mathrm{L}$. & CECA4 & FACU \\
\hline Celtis occidentalis L. & CEOC & FACU \\
\hline Cornus drummondii C.A. Mey. & CODR & FAC \\
\hline
\end{tabular}


Fraxinus americana L.

Fraxinus pennsylvanica Marsh.

Fraxinus quadrangulata Michx.

Fraxinus sp.

Gleditsia tricanthos L.

Juglans cinerea $\mathrm{L}$.

Liriodendron tulipifera $\mathrm{L}$.

Morus rubra L.

Platanus occidentalis $\mathrm{L}$.

Prunus serotina Ehrh.

Quercus rubra L.

Quercus shumardii Buckl.

Robinia pseudoacacia L.

Tilia americana L.

Ulmus rubra Muhl.

\begin{tabular}{l|l} 
FRAM & FACU \\
FRPE & FACW \\
FRQU & NI \\
FRAXI & \\
GLTR & FAC \\
JUCI & FACU \\
LITU & FACU \\
MORU2 & FACU \\
PLOC & FACW \\
PRSE2 & FACU \\
QURU & FACU \\
QUSH & FAC \\
ROPS & FAC \\
TIAM & FACU \\
ULRU & FAC
\end{tabular}

\begin{tabular}{|c|c|c|}
\hline Shrubs & Code & $\begin{array}{l}\text { Wetland } \\
\text { Indicator } \\
\text { Status } \\
\end{array}$ \\
\hline Amelanchier arborea (Michx. f.) Fern. & AMAR3 & FAC \\
\hline Cornus racemosa Lam. & CORA6 & $\mathrm{NI}$ \\
\hline Cornus sp. & CORNU & \\
\hline Euonymus alatus (Thunb.) Sieb. * & EUAL13 & NI \\
\hline Hibiscus syriacus L.** & HISY & NI \\
\hline Hydrangea arborescens $\mathrm{L}$. & HYAR & FACU \\
\hline Hypericum prolificum L. & HYPR & FACU \\
\hline Lagerstroemia indica L.** & LAIN & NI \\
\hline Ligustrum sinense Lour.* & LISI & FACU \\
\hline Lindera benzoin (L.) Blume & LIBE3 & FACW \\
\hline Lonicera maackii (Rupr.) Herder * & LOMA6 & $\mathrm{NI}$ \\
\hline Rosa multiflora Thunb. ex Murr.* & ROMU & FACU \\
\hline Rosa palustris Marsh. & ROPA & OBL \\
\hline Rubus spp. & RUBUS & \\
\hline Symphoricarpos orbiculatus Moench & SYOR & UPL \\
\hline Viburnum acerifolium L. & VIAC & UPL \\
\hline
\end{tabular}

Non-native species indicated with a $\left(^{*}\right)$ if naturalized, and a $\left({ }^{* *}\right)$ if nonnaturalized. Naturalized, exotic species successfully reproduce and maintain viable populations without human intervention.

Plants were identified to genus group if species could not be determined. 
Appendix Figure 5: Woody species abundance and diversity plotted against \% impervious surface at the subcatchment (SC) scale and within 1-km of the site as well as streambank height for all sites along first order streams.
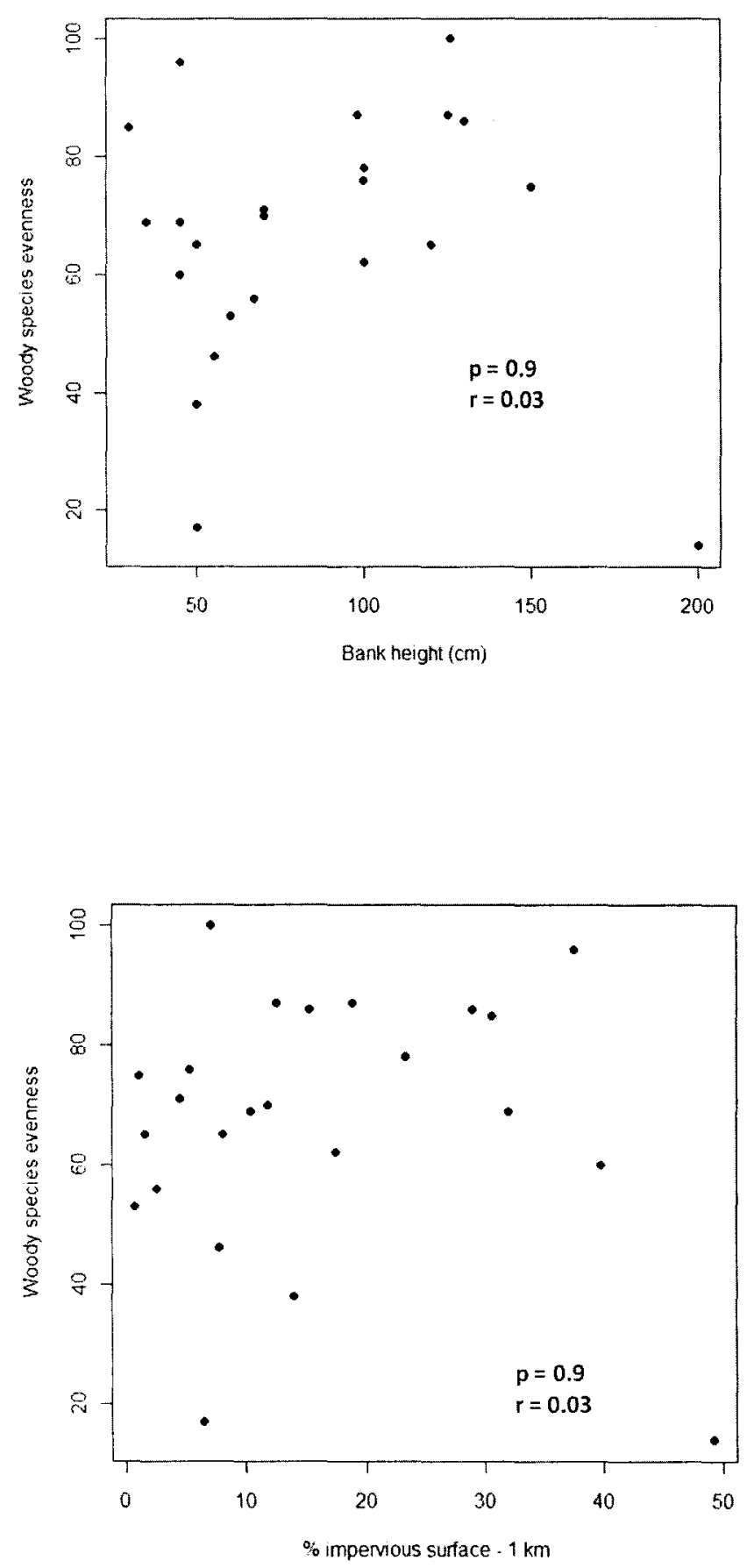


\section{Appendix Figure 5 (continued)}
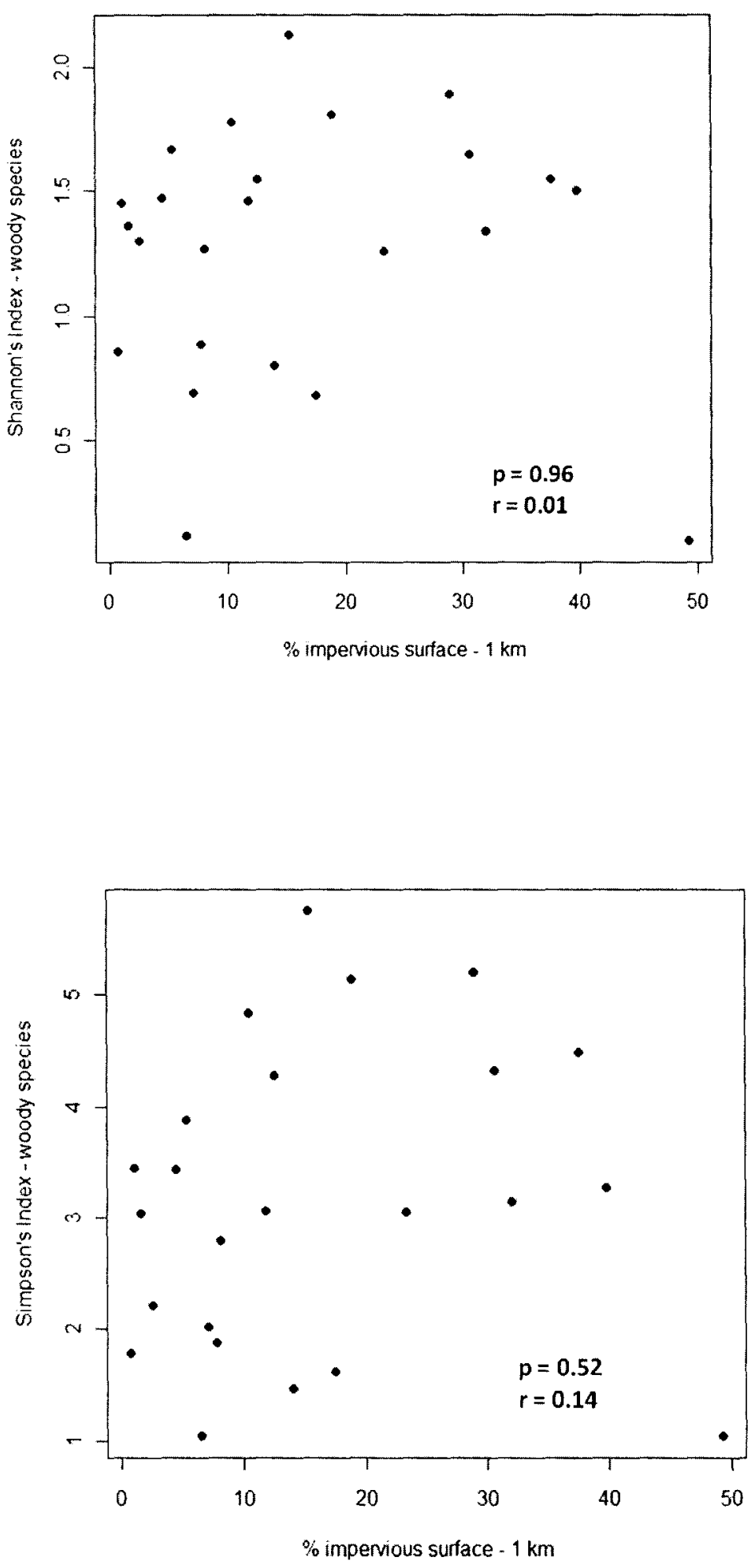


\section{Appendix Figure 5 (continued)}
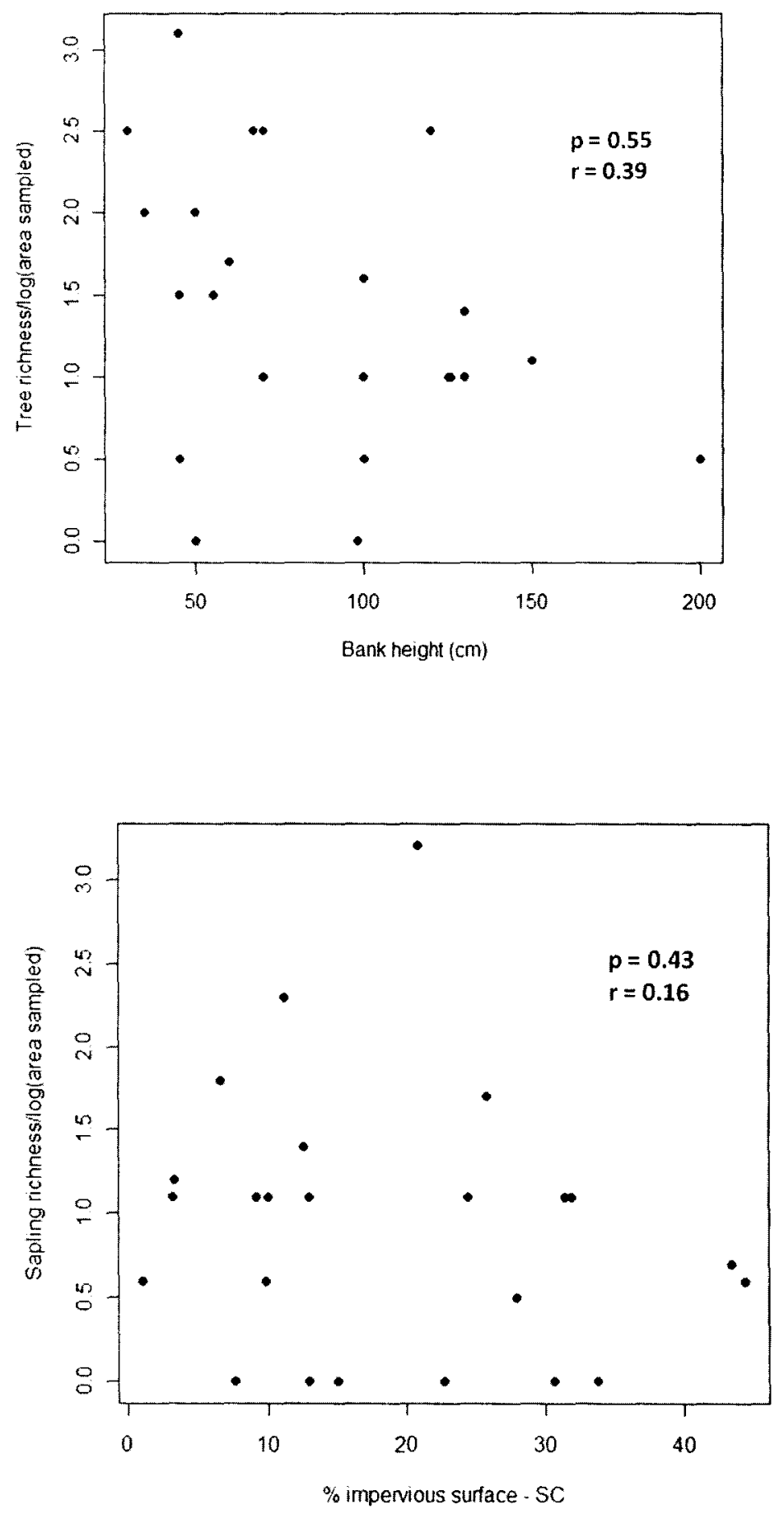


\section{Appendix Figure 5 (continued)}
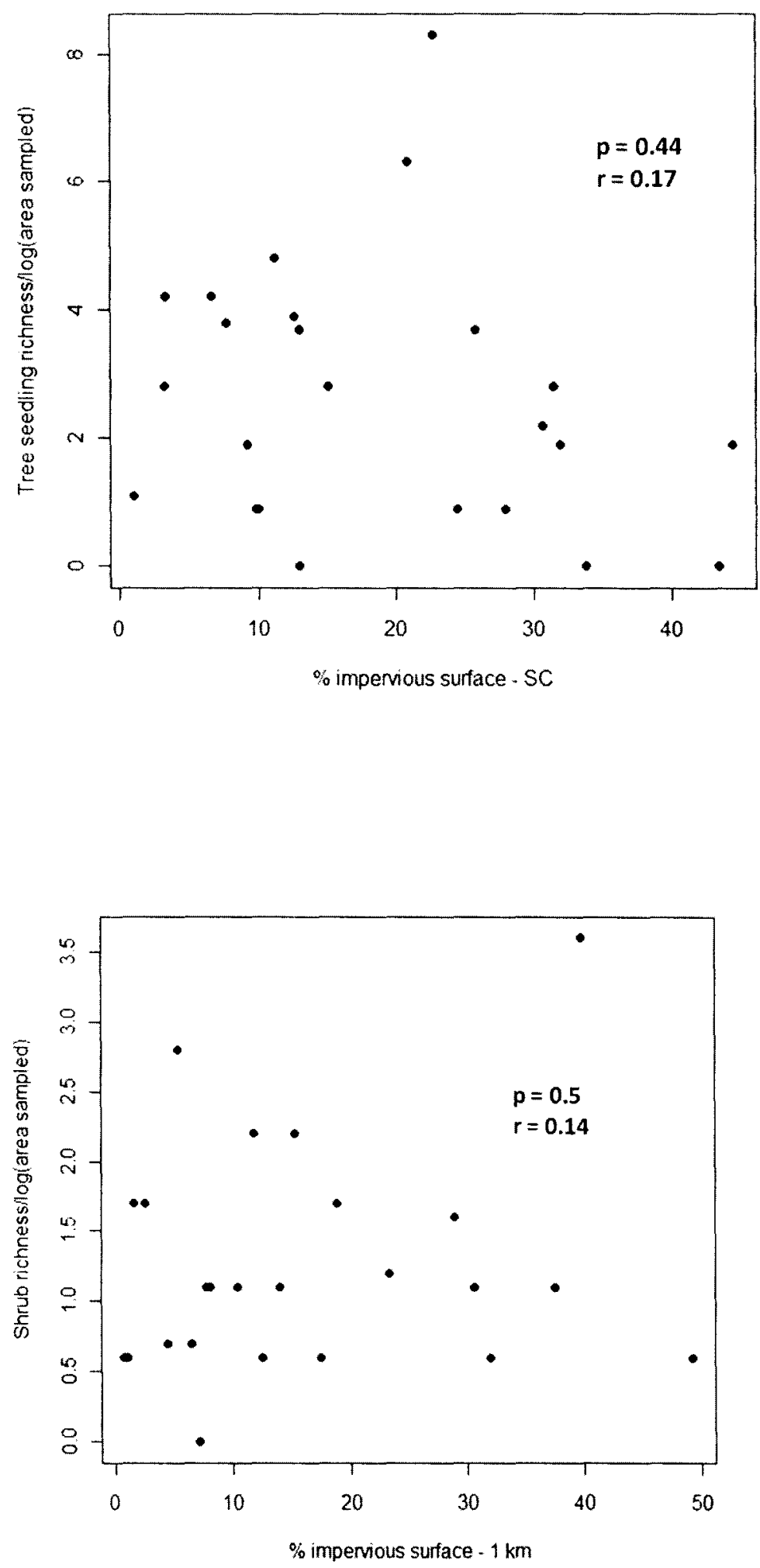


\section{Appendix Figure 5 (continued)}
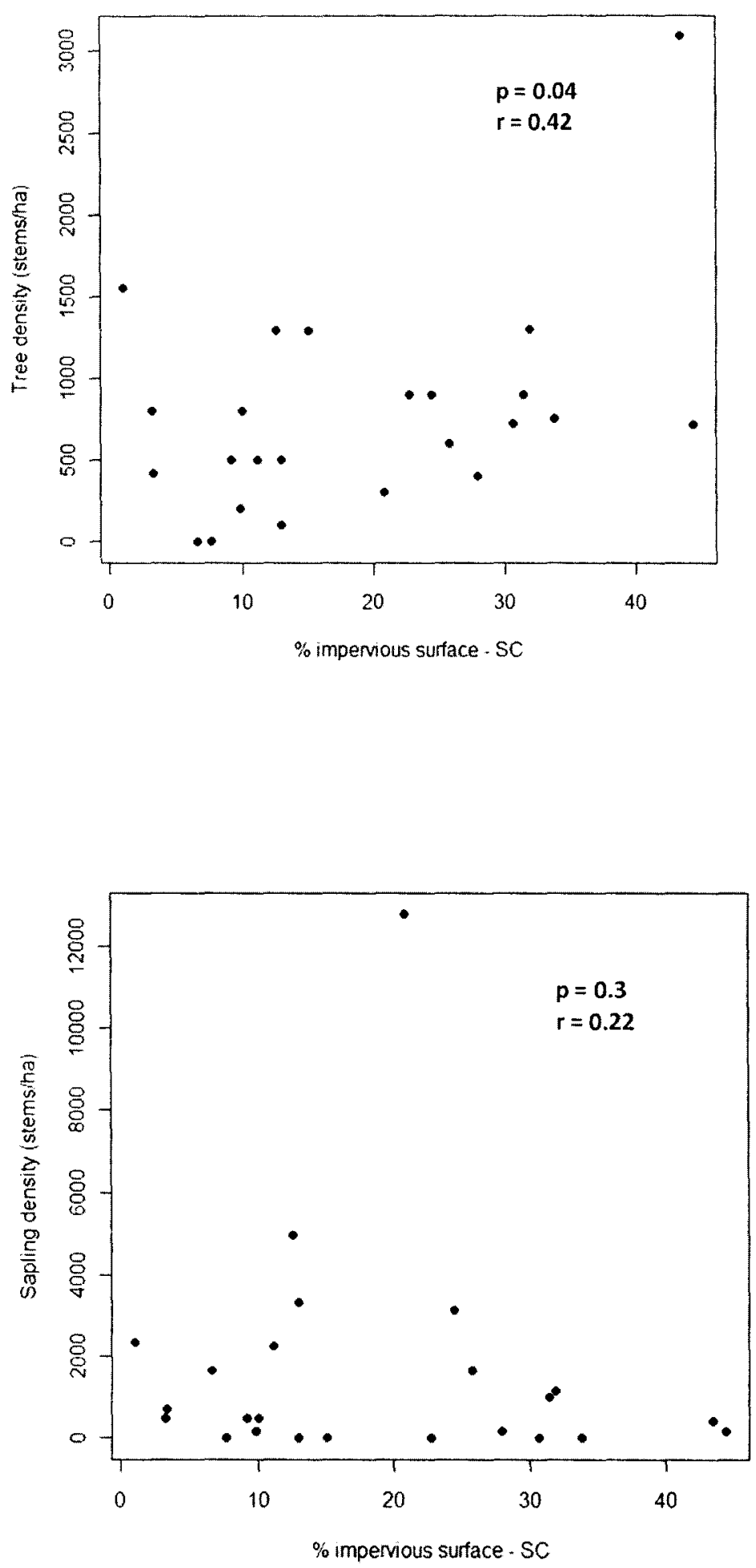


\section{Appendix Figure 5 (continued)}
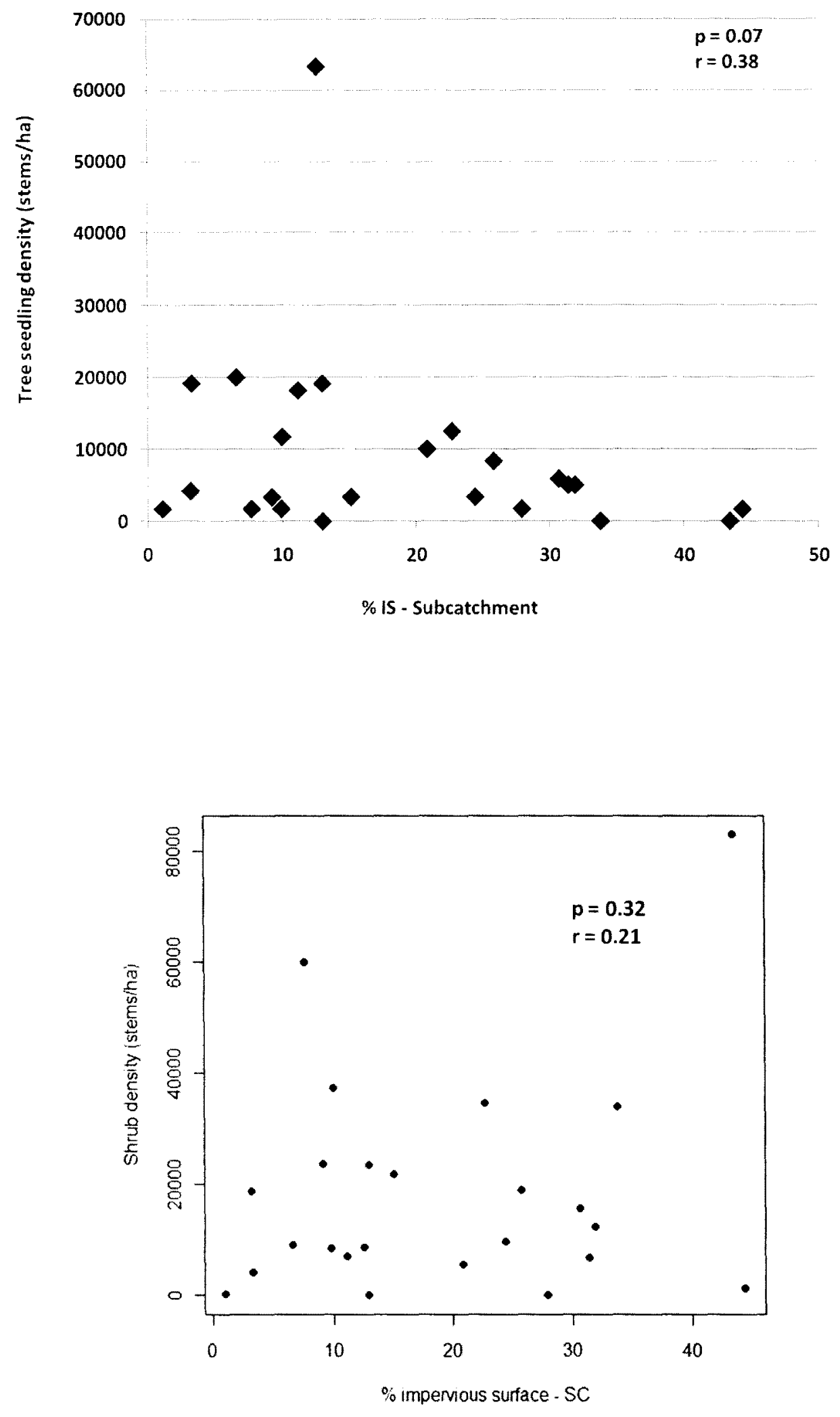
Appendix Figure 6: Total groundcover, herb, and woody vine abundance ( $\%$ cover), as well as proportion exotic herb and woody vine species richness and abundance, vs. $\%$ impervious surface at the subcatchment scale.
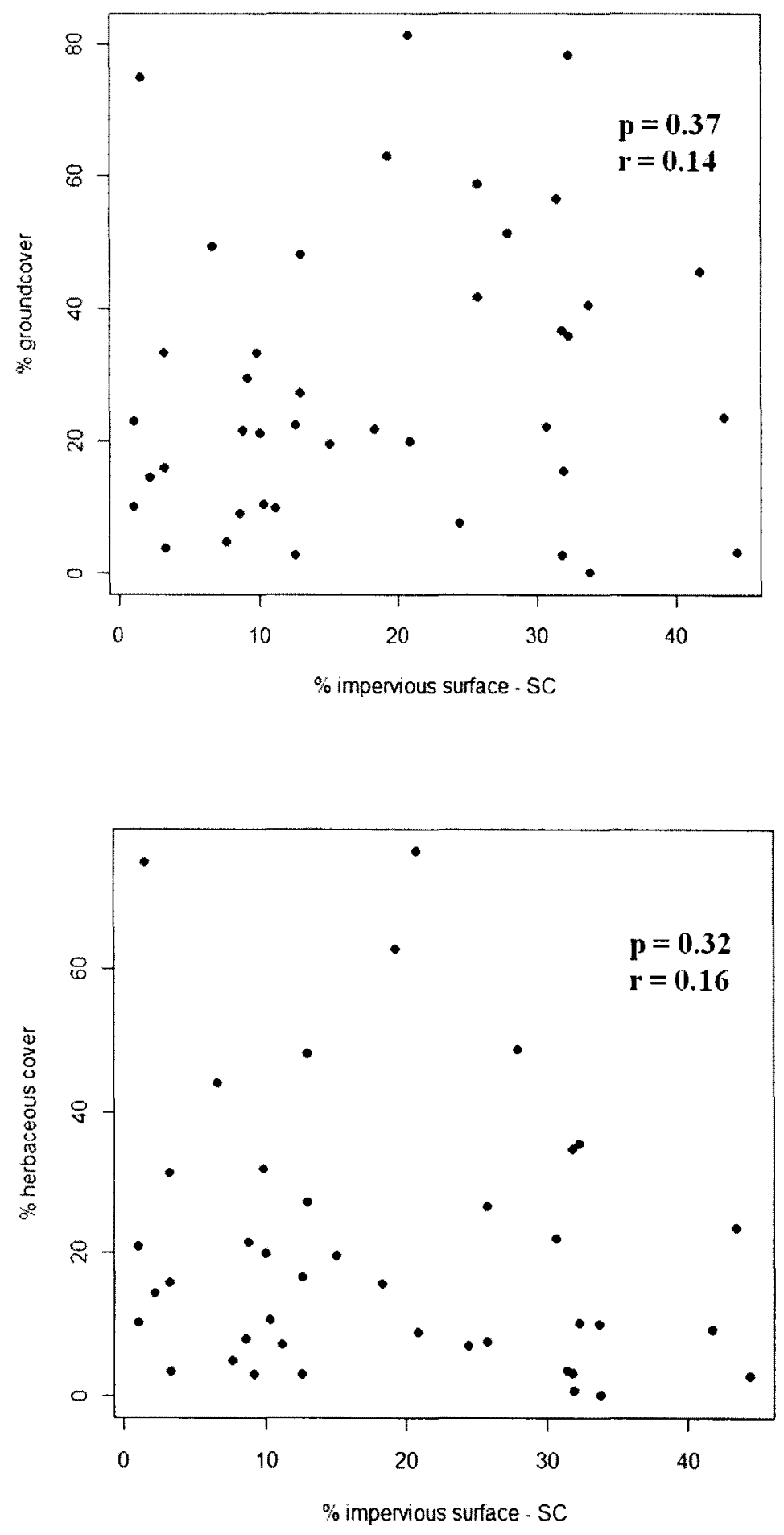


\section{Appendix Figure 6 (continued)}
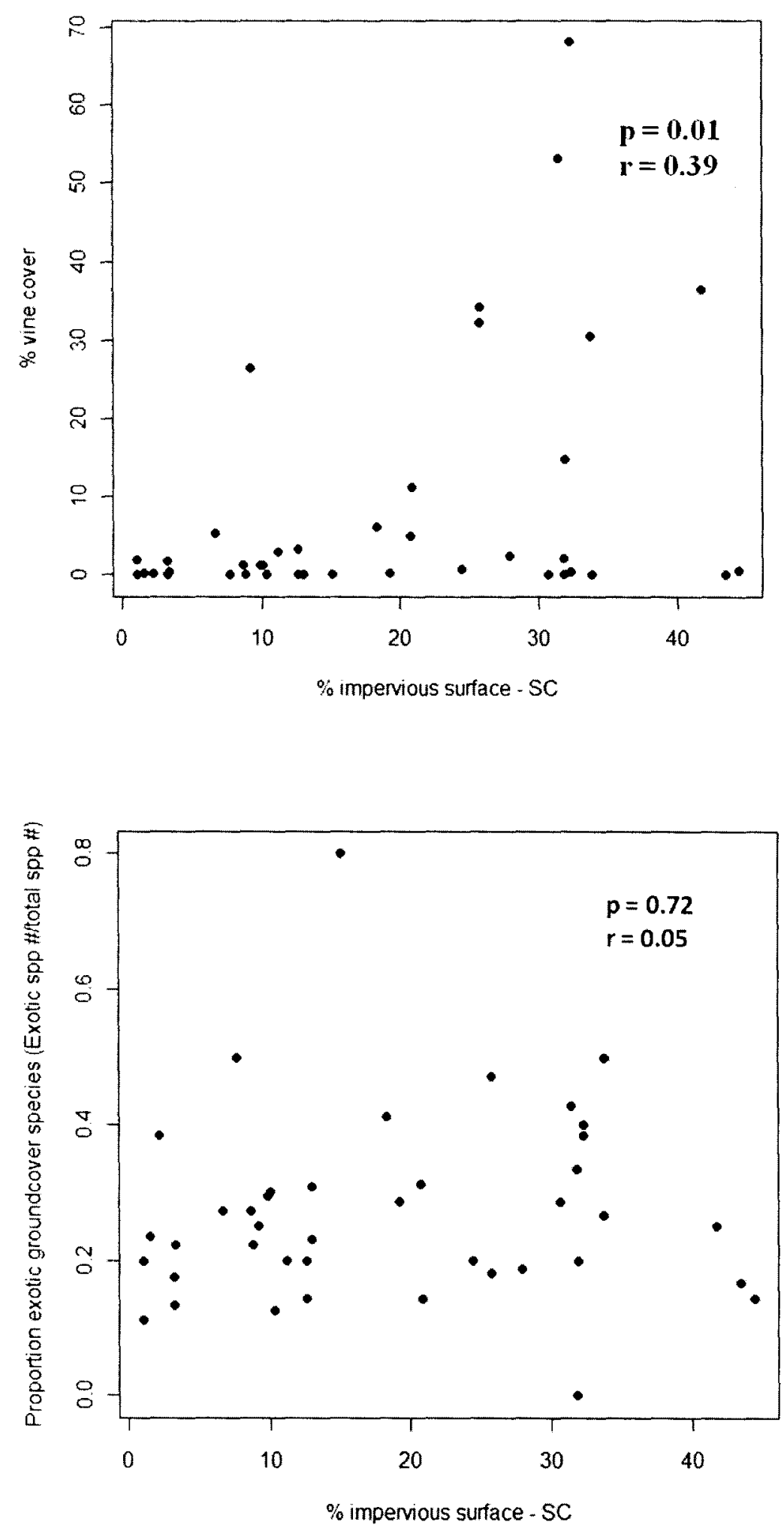
Appendix Figure 6 (continued)
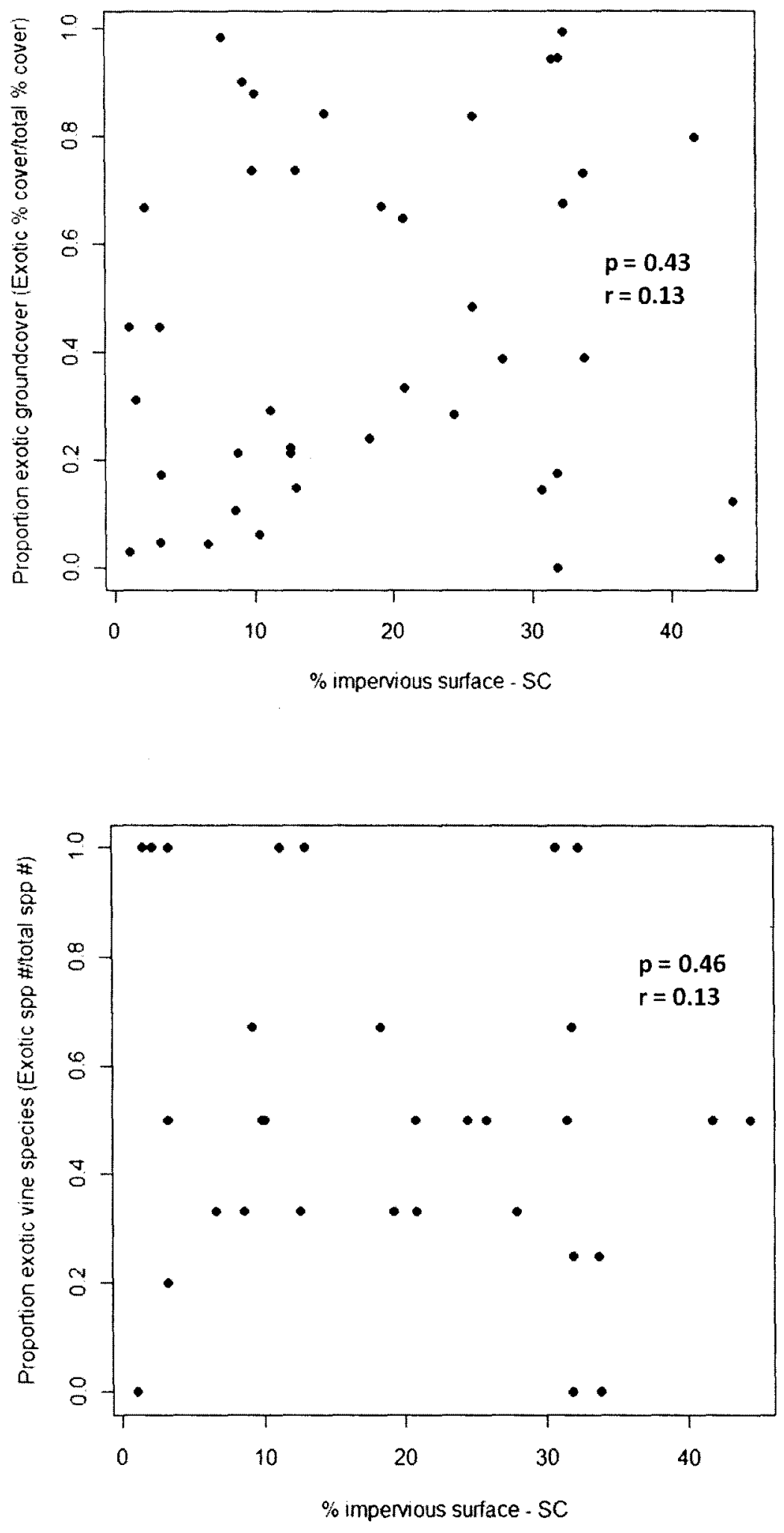


\section{Appendix Figure 6 (continued)}
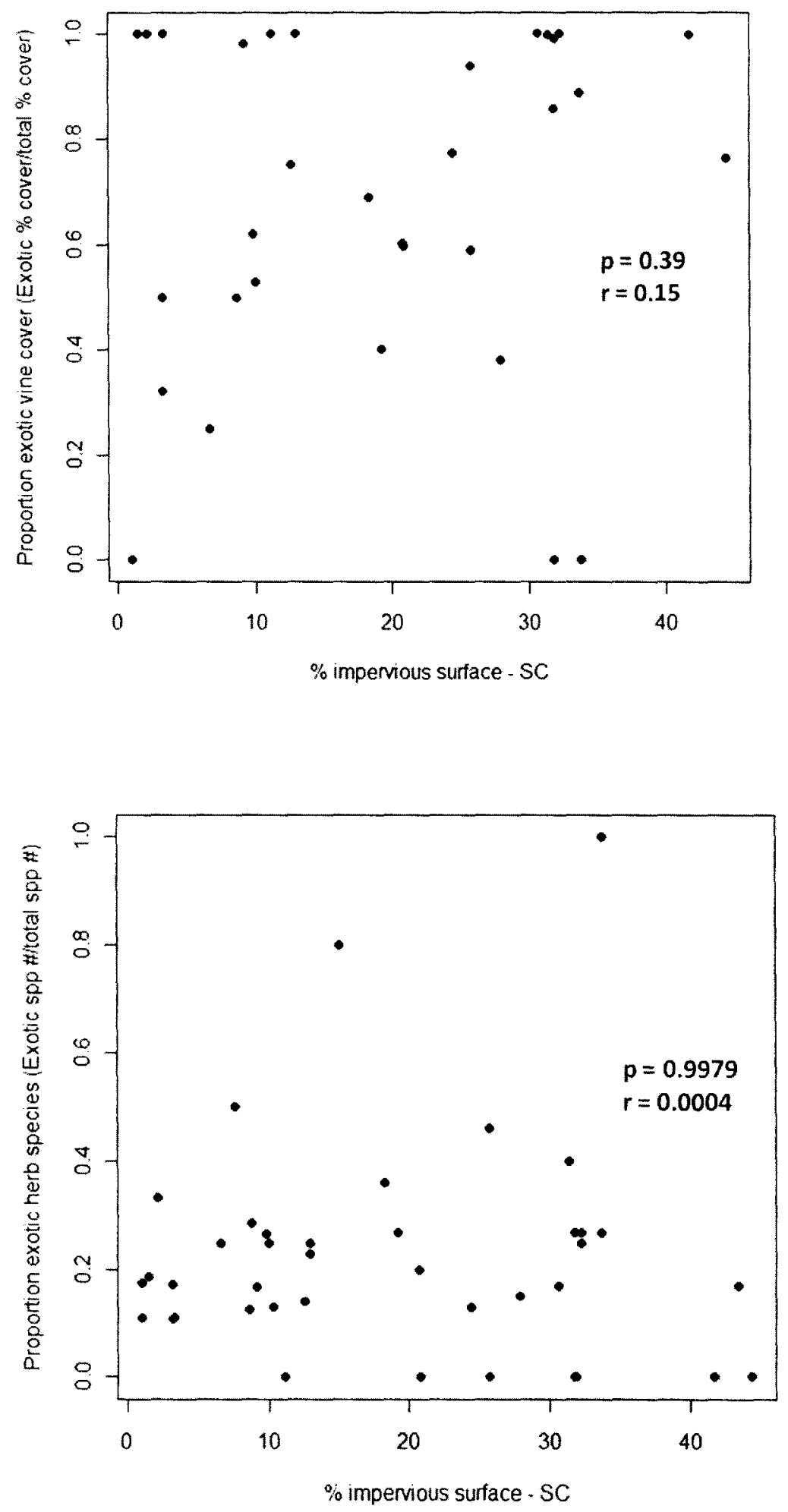


\section{Appendix Figure 6 (continued)}

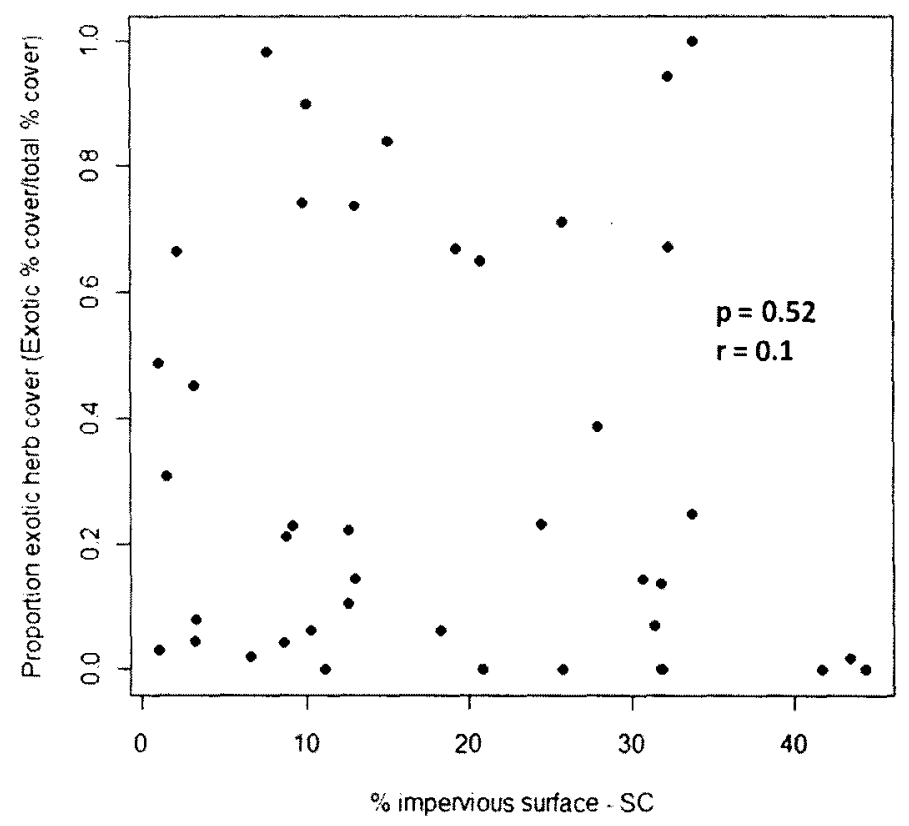


Appendix Figure 7: Groundcover (herb and woody vine) species evenness vs. bank height and groundcover abundances (\% cover) vs. \% impervious surface at the subcatchment scale within riparian zones along first order streams.
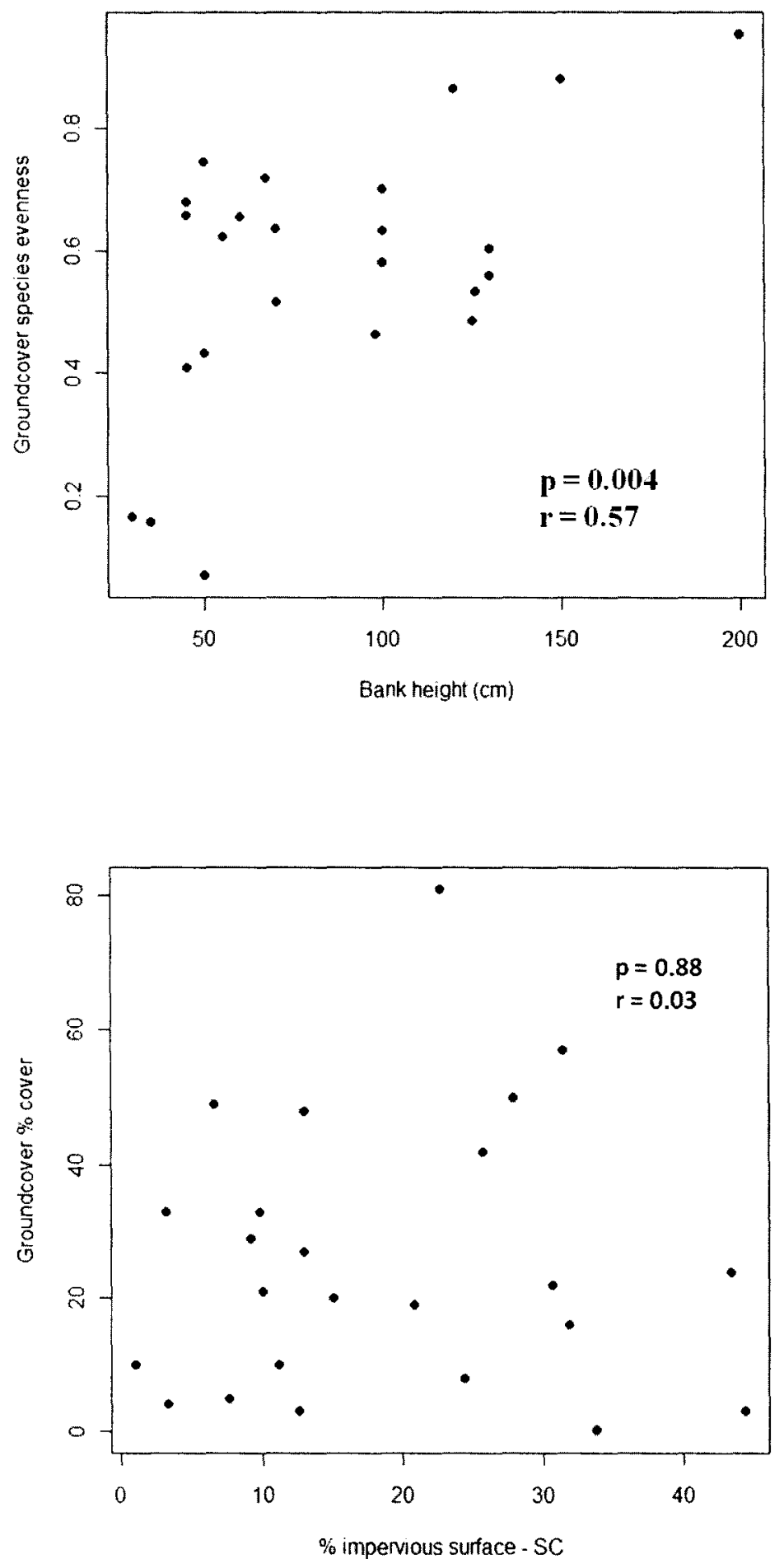
Appendix Figure 7 (cont.)
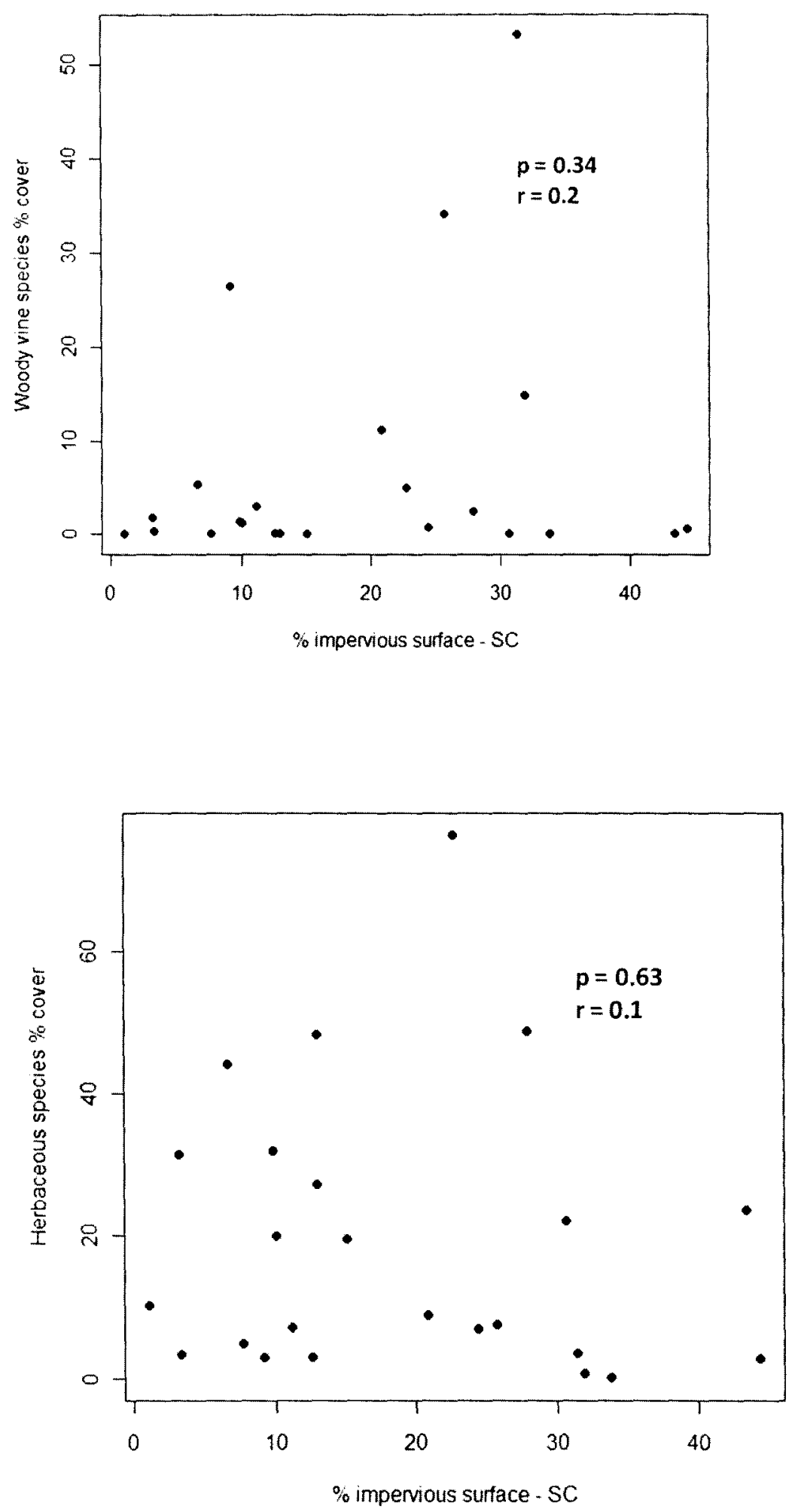
Appendix Table 8: Sapling species within 41 riparian sites in Jefferson and Oldham counties in Kentucky. Site names refer to the watershed in which the site was located, where $\mathrm{B}=$ Middle Fork Beargrass, $\mathrm{G}=$ Goose, and $\mathrm{H}=$ South Fork Harrods Creek watersheds. Relative importance values (IV) were calculated from relative density and basal area by land cover category. Land use categories for each site were determined by the proportion of impervious surface (IS) within the subcatchment in which the site was located. Urban $\geq 30 \%$ IS, Rural $\leq 10 \%$ IS, and Suburban is between 10 and $30 \%$ IS.

\begin{tabular}{|c|c|c|c|c|c|}
\hline Site & $\begin{array}{l}\text { Land- } \\
\text { cover } \\
\text { category }\end{array}$ & Sapling Species Names & Freq. & $\begin{array}{l}\text { Density } \\
\text { (stems/ } \\
\text { ha) }\end{array}$ & IV \\
\hline $\mathrm{G} 2$ & Rural & Celtis occidentalis L. & 0.33 & 222 & 133 \\
\hline $\mathrm{G} 2$ & Rural & Fraxinus americana $\mathrm{L}$. & 0.17 & 111 & 67 \\
\hline G4 & Rural & Acer negundo L. & 0.33 & 208 & 38 \\
\hline G4 & Rural & Celtis occidentalis L. & 0.67 & 625 & 88 \\
\hline G4 & Rural & Robinia pseudoacacia L. & 0.33 & 833 & 75 \\
\hline $\mathrm{H} 1$ & Rural & Asimina triloba (L.) Dunal & 0.33 & 333 & 117 \\
\hline $\mathrm{H} 1$ & Rural & Ulmus rubra Muhl. & 0.33 & 167 & 83 \\
\hline $\mathrm{H} 11$ & Rural & Acer negundo L. & 0.33 & 333 & 117 \\
\hline H11 & Rural & Ulmus rubra Muhl. & 0.33 & 167 & 83 \\
\hline $\mathrm{H} 12$ & Rural & Acer negundo L. & 0.17 & 83 & 100 \\
\hline $\mathrm{H} 12$ & Rural & Juglans nigra L. & 0.17 & 83 & 100 \\
\hline $\mathrm{H} 14$ & Rural & Asimina triloba (L.) Dunal & 0.33 & 500 & 200 \\
\hline $\mathrm{H} 5$ & Rural & Aesculus glabra Willd. & 0.17 & 83 & 50 \\
\hline $\mathrm{H} 5$ & Rural & Asimina triloba (L.) Dunal & 0.50 & 250 & 150 \\
\hline H6 & Rural & Asimina triloba (L.) Dunal & 0.33 & 476 & 117 \\
\hline H6 & Rural & Fraxinus americana $\mathrm{L}$. & 0.33 & 238 & 83 \\
\hline $\mathrm{H} 7$ & Rural & Asimina triloba (L.) Dunal & 1.00 & 2368 & 200 \\
\hline $\mathrm{H} 8$ & Rural & Aesculus glabra Willd. & 0.33 & 500 & 125 \\
\hline $\mathrm{H} 8$ & Rural & Asimina triloba (L.) Dunal & 0.17 & 83 & 38 \\
\hline $\mathrm{H} 8$ & Rural & Ulmus rubra Muhl. & 0.17 & 83 & 38 \\
\hline B11 & Suburban & Carya cordiformis (Wangenh.) K. Koch & 0.33 & 556 & 29 \\
\hline B 11 & Suburban & Cornus sp. & 0.33 & 1667 & 38 \\
\hline B 11 & Suburban & Fraxinus americana $\mathrm{L}$. & 0.33 & 556 & 29 \\
\hline B11 & Suburban & Staphylea trifolia $\mathrm{L}$. & 0.33 & 10000 & 103 \\
\hline G10 & Suburban & Celtis occidentalis L. & 0.33 & 167 & 200 \\
\hline G11 & Suburban & Acer negundo L. & 1.00 & 2333 & 148 \\
\hline G11 & Suburban & Prunus serotina Ehrh. & 0.33 & 167 & 26 \\
\hline G12 & Suburban & Acer saccharum Marsh. & 1.00 & 1167 & 130 \\
\hline G12 & Suburban & Celtis occidentalis L. & 0.33 & 333 & 40 \\
\hline G12 & Suburban & Fraxinus americana L. & 0.33 & 167 & 30 \\
\hline
\end{tabular}




\section{Appendix Table 8 (cont.)}

\begin{tabular}{l|l|l|c|c|c} 
G3 & Suburban & Fraxinus americana L. & 0.33 & 167 & 83 \\
G3 & Suburban & Ulmus rubra Muhl. & 0.33 & 333 & 117 \\
G6 & Suburban & Aesculus glabra Willd. & 0.33 & 167 & 30 \\
G6 & Suburban & Asimina triloba (L.) Dunal & 1.00 & 3167 & 170 \\
H10 & Suburban & Carya cordiformis (Wangenh.) K. Koch & 0.33 & 175 & 18 \\
H10 & Suburban & Celtis occidentalis L. & 0.33 & 175 & 18 \\
H10 & Suburban & Cercis canadensis L. & 0.33 & 175 & 18 \\
H10 & Suburban & Fraxinus quadrangulata Michx. & 0.67 & 351 & 35 \\
H10 & Suburban & Quercus rubra L. & 0.33 & 175 & 18 \\
H10 & Suburban & Tilia americana L. & 0.33 & 175 & 18 \\
H10 & Suburban & Ulmus rubra Muhl. & 1.00 & 1053 & 76 \\
H2 & Suburban & Acer negundo L. & 0.33 & 167 & 55 \\
H2 & Suburban & Asimina triloba (L.) Dunal & 1.00 & 3000 & 145 \\
H4 & Suburban & Asimina triloba (L.) Dunal & 0.67 & 2917 & 108 \\
H4 & Suburban & Fraxinus pennsylvanica Marsh. & 0.67 & 2083 & 92 \\
B1 & Urban & Acer negundo L. & 0.50 & 833 & 143 \\
B1 & Urban & Morus rubra L. & 0.33 & 167 & 57 \\
B13 & Urban & Acer negundo L. & 0.33 & 278 & 200 \\
B14 & Urban & Celtis occidentalis L. & 0.33 & 667 & 117 \\
B14 & Urban & Fraxinus americana L. & 0.33 & 333 & 83 \\
B2 & Urban & Celtis occidentalis L. & 1.00 & 1500 & 200 \\
B3 & Urban & Acer saccharum Marsh. & 0.67 & 1000 & 53 \\
B3 & Urban & Carya cordiformis (Wangenh.) K. Koch & 0.33 & 167 & 20 \\
B3 & Urban & Celtis occidentalis L. & 1.00 & 4000 & 127 \\
B6 & Urban & Fraxinus quadrangulata Michx. & 0.33 & 417 & 200 \\
B7 & Urban & Acer negundo L. & 0.11 & 56 & 22 \\
B7 & Urban & Celtis occidentalis L. & 0.11 & 56 & 22 \\
B7 & Urban & Cornus sericea L. ssp. sericea & 0.11 & 222 & 45 \\
B7 & Urban & Fraxinus pennsylvanica Marsh. & 0.22 & 278 & 67 \\
B7 & Urban & Maclura pomifera (Raf.) Schneid. & 0.11 & 56 & 22 \\
B7 & Urban & Thuja occidentalis & 0.11 & 56 & 22 \\
B8 & Urban & Morus rubra L. & 0.33 & 167 & 200
\end{tabular}


Appendix Table 9: Shrub species within 41 riparian sites in Jefferson and Oldham counties in Kentucky. Site names refer to the watershed in which the site was located, where $\mathrm{B}=$ Middle Fork Beargrass, $\mathrm{G}=$ Goose, and $\mathrm{H}=$ South Fork Harrods Creek watersheds. Relative importance values (IV) were calculated from relative density and basal area by land cover category. Land use categories for each site were determined by the proportion of impervious surface (IS) within the subcatchment in which the site was located. Urban $\geq 30 \%$ IS, Rural $\leq 10 \%$ IS, and Suburban is between 10 and $30 \%$ IS.

\begin{tabular}{|c|c|c|c|c|c|}
\hline Site & $\begin{array}{l}\text { Land } \\
\text { use } \\
\text { category } \\
\end{array}$ & Shrub Species Names & Freq. & $\begin{array}{l}\text { Density } \\
\text { (stems/ } \\
\text { ha) }\end{array}$ & IV \\
\hline $\mathrm{G} 2$ & Rural & Euonymus alatus (Thunb.) Sieb. & 0.67 & 444 & 36 \\
\hline $\mathrm{G} 2$ & Rural & Ligustrum sinense Lour. & 0.67 & 2667 & 53 \\
\hline G2 & Rural & Lonicera maackii (Rupr.) Herder & 0.67 & 7778 & 94 \\
\hline G4 & Rural & Lonicera maackii (Rupr.) Herder & 1.00 & 6875 & 200 \\
\hline G7 & Rural & Hydrangea arborescens $\mathrm{L}$. & 0.17 & 1667 & 45 \\
\hline G7 & Rural & Lindera benzoin (L.) Blume & 0.67 & 5000 & 155 \\
\hline $\mathrm{H} 1$ & Rural & Lindera benzoin (L.) Blume & 1.00 & 7667 & 129 \\
\hline $\mathrm{H} 1$ & Rural & Lonicera maackii (Rupr.) Herder & 0.33 & 1167 & 29 \\
\hline H11 & Rural & Ligustrum sinense Lour. & 1.00 & 16333 & 174 \\
\hline H11 & Rural & Lindera benzoin (L.) Blume & 0.33 & 167 & 26 \\
\hline H12 & Rural & Cornus racemosa Lam. & 0.33 & 583 & 17 \\
\hline $\mathrm{H} 12$ & Rural & Lonicera maackii (Rupr.) Herder & 0.67 & 1167 & 34 \\
\hline H12 & Rural & Rubus spp. & 0.42 & 8333 & 72 \\
\hline $\mathrm{H} 12$ & Rural & Symphoricarpos orbiculatus Moench & 0.33 & 3333 & 35 \\
\hline $\mathrm{H} 12$ & Rural & Viburnum acerifolium $\mathrm{L}$. & 0.08 & 833 & 9 \\
\hline H13 & Rural & Symphoricarpos orbiculatus Moench & 1.00 & 60000 & 200 \\
\hline H14 & Rural & Lindera benzoin (L.) Blume & 0.33 & 667 & 200 \\
\hline H3 & Rural & Symphoricarpos orbiculatus Moench & 0.33 & 167 & 200 \\
\hline H5 & Rural & Hydrangea arborescens $\mathrm{L}$. & 0.08 & 833 & 13 \\
\hline H5 & Rural & Ligustrum sinense Lour. & 0.08 & 1667 & 22 \\
\hline H5 & Rural & Lindera benzoin (L.) Blume & 1.00 & 2167 & 81 \\
\hline H5 & Rural & Lonicera maackii (Rupr.) Herder & 0.25 & 1667 & 32 \\
\hline H6 & Rural & Lindera benzoin (L.) Blume & 1.00 & 4286 & 200 \\
\hline $\mathrm{H} 7$ & Rural & Lindera benzoin (L.) Blume & 0.33 & 263 & 200 \\
\hline $\mathrm{H} 8$ & Rural & Ligustrum sinense Lour. & 0.67 & 833 & 33 \\
\hline $\mathrm{H} 8$ & Rural & Lindera benzoin (L.) Blume & 0.83 & 3750 & 69 \\
\hline $\mathrm{H} 8$ & Rural & Lonicera maackii (Rupr.) Herder & 0.83 & 5000 & 82 \\
\hline $\mathrm{H} 9$ & Rural & Lonicera maackii (Rupr.) Herder & 1.00 & 7333 & 200 \\
\hline B11 & Suburban & Euonymus alatus (Thunb.) Sieb. & 0.33 & 3333 & 110 \\
\hline
\end{tabular}


Appendix Table 9 (cont.)

\begin{tabular}{|c|c|c|c|c|}
\hline 311 & Suburban & Lonicera maackii (Rupr.) Herder & 0.33 & 2222 \\
\hline B12 & Suburban & $\begin{array}{l}\text { Amelanchier arborea (Michx. f.) } \\
\text { Fern. }\end{array}$ & 0.33 & 833 \\
\hline 12 & Suburban & Ligustrum sinense Lour. & 1.00 & 10167 \\
\hline 12 & Suburban & Lindera benzoin (L.) Blume & 1.00 & 3167 \\
\hline 12 & Suburban & Lonicera maackii (Rupr.) Herder & 0.33 & 500 \\
\hline G1 & Suburban & Euonymus alatus (Thunb.) Sieb. & 0.67 & 833 \\
\hline G1 & Suburban & Lonicera maackii (Rupr.) Herder & 1.00 & 20167 \\
\hline G10 & Suburban & Euonymus alatus (Thunb.) Sieb. & 0.33 & 167 \\
\hline 11 & Suburban & Euonymus alatus (Thunb.) Sieb. & 0.67 & 667 \\
\hline 11 & Suburban & Hibiscus syriacus L. & 0.08 & 1667 \\
\hline 11 & Suburban & Lonicera maackii (Rupr.) Herder & 1.00 & 3833 \\
\hline G12 & Suburban & Ligustrum sinense Lour. & 1.00 & 3167 \\
\hline G12 & Suburban & Lonicera maackii (Rupr.) Herder & 0.67 & 9167 \\
\hline 14 & Suburban & Lonicera maackii (Rupr.) Herder & 0.33 & 3333 \\
\hline $\mathrm{s} 14$ & Suburban & Rubus spp. & 0.33 & 3333 \\
\hline G3 & Suburban & Cornus racemosa Lam. & 0.33 & 1333 \\
\hline G3 & Suburban & Ligustrum sinense Lour. & 0.33 & 2333 \\
\hline G6 & Suburban & Ligustrum sinense Lour. & 1.00 & 20833 \\
\hline G6 & Suburban & Lindera benzoin (L.) Blume & 0.33 & 167 \\
\hline H10 & Suburban & Ligustrum sinense Lour. & 0.33 & 175 \\
\hline $\mathrm{H} 10$ & Suburban & Lonicera maackii (Rupr.) Herder & 0.67 & 2281 \\
\hline $\mathrm{H} 2$ & Suburban & Euonymus alatus (Thunb.) Sieb. & 0.08 & 833 \\
\hline $\mathrm{H} 2$ & Suburban & Ligustrum sinense Lour. & 0.33 & 167 \\
\hline $\mathrm{H} 2$ & Suburban & Lindera benzoin (L.) Blume & 1.00 & 5500 \\
\hline $\mathrm{H} 4$ & Suburban & Lindera benzoin (L.) Blume & 0.67 & 2083 \\
\hline B1 & Urban & Lonicera maackii (Rupr.) Herder & 0.33 & 333 \\
\hline $\mathrm{B} 13$ & Urban & Lonicera maackii (Rupr.) Herder & 0.67 & 6111 \\
\hline B14 & Urban & Lindera benzoin (L.) Blume & 1.00 & 3667 \\
\hline B14 & Urban & Lonicera maackii (Rupr.) Herder & 0.67 & 2333 \\
\hline $\mathrm{B} 2$ & Urban & Euonymus alatus (Thunb.) Sieb. & 0.33 & 333 \\
\hline B2 & Urban & Hypericum prolificum $\mathrm{L}$. & 0.33 & 167 \\
\hline $\mathrm{B} 2$ & Urban & Ligustrum sinense Lour. & 0.67 & 4667 \\
\hline B2 & Urban & Lindera benzoin (L.) Blume & 0.67 & 833 \\
\hline B2 & Urban & Lonicera maackii (Rupr.) Herder & 1.00 & 4833 \\
\hline B3 & Urban & Lonicera maackii (Rupr.) Herder & 1.00 & 12333 \\
\hline B4 & Urban & Lonicera maackii (Rupr.) Herder & 1.00 & 13000 \\
\hline B5 & Urban & Lonicera maackii (Rupr.) Herder & 0.67 & 6833 \\
\hline B6 & Urban & Cornus sp. & 0.33 & 9583 \\
\hline B6 & Urban & Hibiscus syriacus L. & 0.67 & 7917 \\
\hline
\end{tabular}




\begin{tabular}{l|l|l|c|c|c} 
Appendix Table 9 (cont.) \\
B6 & Urban & Lagerstroemia indica L. & 0.33 & 5000 & 25 \\
B6 & Urban & Lonicera maackii (Rupr.) Herder & 1.00 & 25417 & 96 \\
B7 & Urban & Lonicera maackii (Rupr.) Herder & 1.00 & 12778 & 156 \\
B7 & Urban & Rosa palustris Marsh. & 0.56 & 1167 & 44 \\
B8 & Urban & Euonymus alatus (Thunb.) Sieb. & 0.33 & 500 & 118 \\
B8 & Urban & Lonicera maackii (Rupr.) Herder & 0.08 & 833 & 83 \\
B9 & Urban & Lonicera maackii (Rupr.) Herder & 1.00 & 6500 & 200 \\
G8 & Urban & Lindera benzoin (L.) Blume & 0.67 & 1000 & 67 \\
G8 & Urban & Lonicera maackii (Rupr.) Herder & 0.67 & 4750 & 133
\end{tabular}


Appendix Table 10: Tree seedling species within 41 riparian sites in Jefferson and Oldham counties in Kentucky. Site names refer to the watershed in which the site was located, where B = Middle Fork Beargrass, $\mathrm{G}=$ Goose, and $\mathrm{H}=$ South Fork Harrods Creek watersheds. Relative importance values (IV) were calculated from relative density and basal area by land cover category. Land use categories for each site were determined by the proportion of impervious surface (IS) within the subcatchment in which the site was located. Urban $\geq 30 \%$ IS, Rural $\leq 10 \%$ IS, and Suburban is between 10 and $30 \%$ IS.

\begin{tabular}{|c|c|c|c|c|c|}
\hline Site & $\begin{array}{l}\text { Land } \\
\text { use } \\
\text { category }\end{array}$ & Tree seedling species names & Freq. & $\begin{array}{l}\text { Density } \\
\text { (stems/ } \\
\text { ha) }\end{array}$ & IV \\
\hline $\mathrm{G} 2$ & Rural & Acer negundo L. & 0.0011 & 2778 & 89 \\
\hline $\mathrm{G} 2$ & Rural & Acer saccharum Marsh. & 0.0006 & 556 & 28 \\
\hline $\mathrm{G} 2$ & Rural & Celtis occidentalis L. & 0.0017 & 1667 & 83 \\
\hline G4 & Rural & Acer negundo L. & 0.4444 & 6667 & 67 \\
\hline G4 & Rural & Acer saccharum Marsh. & 0.4444 & 5556 & 61 \\
\hline G4 & Rural & Celtis occidentalis L. & 0.3333 & 6667 & 58 \\
\hline G4 & Rural & Platanus occidentalis L. & 0.1111 & 1111 & 14 \\
\hline $\mathrm{H} 1$ & Rural & Asimina triloba (L.) Dunal & 0.0833 & 833 & 45 \\
\hline $\mathrm{H} 1$ & Rural & Carya cordiformis (Wangenh.) K. Koch & 0.0833 & 833 & 45 \\
\hline H1 & Rural & Fraxinus americana $\mathrm{L}$. & 0.0833 & 833 & 45 \\
\hline $\mathrm{H} 1$ & Rural & Ulmus rubra Muhl. & 0.0833 & 1667 & 65 \\
\hline H11 & Rural & Acer negundo L. & 0.2500 & 5000 & 161 \\
\hline H11 & Rural & Asimina triloba (L.) Dunal & 0.0833 & 833 & 39 \\
\hline $\mathrm{H} 12$ & Rural & Celtis occidentalis L. & 0.1667 & 833 & 200 \\
\hline H13 & Rural & Acer negundo L. & 0.1667 & 1667 & 200 \\
\hline $\mathrm{H} 14$ & Rural & Acer negundo $\mathrm{L}$. & 0.0833 & 417 & 100 \\
\hline $\mathrm{H} 14$ & Rural & Aesculus glabra Willd. & 0.0833 & 417 & 100 \\
\hline $\mathrm{H} 5$ & Rural & Acer sp. & 0.0004 & 417 & 25 \\
\hline H5 & Rural & Acer negundo L. & 0.0004 & 417 & 25 \\
\hline $\mathrm{H} 5$ & Rural & Asimina triloba (L.) Dunal & 0.0017 & 1667 & 100 \\
\hline $\mathrm{H} 5$ & Rural & Robinia pseudoacacia L. & 0.0004 & 417 & 25 \\
\hline H5 & Rural & Ulmus rubra Muhl. & 0.0004 & 417 & 25 \\
\hline H6 & Rural & Acer saccharum Marsh. & 0.4444 & 7778 & 57 \\
\hline H6 & Rural & Celtis occidentalis L. & 0.2222 & 2222 & 22 \\
\hline H6 & Rural & Fraxinus americana L. & 0.7778 & 13333 & 99 \\
\hline H6 & Rural & Fraxinus quadrangulata Michx. & 0.2222 & 2222 & 22 \\
\hline $\mathrm{H} 7$ & Rural & Asimina triloba (L.) Dunal & 0.1250 & 1250 & 100 \\
\hline $\mathrm{H} 7$ & Rural & Juglans cinerea L. & 0.1250 & 1250 & 100 \\
\hline $\mathrm{H} 8$ & Rural & Acer sp. & 0.1667 & 833 & 100 \\
\hline $\mathrm{H} 8$ & Rural & Aesculus glabra Willd. & 0.0833 & 417 & 50 \\
\hline
\end{tabular}




\begin{tabular}{|c|c|c|c|c|c|}
\hline & & Appendix Table 10 (cont.) & & & \\
\hline H8 & Rural & Celtis occidentalis L. & 0.0833 & 417 & 50 \\
\hline H9 & Rural & Fraxinus americana $\mathrm{L}$. & 0.5000 & 8889 & 140 \\
\hline H9 & Rural & Quercus rubra L. & 0.3333 & 2222 & 60 \\
\hline $\mathrm{B} 10$ & Suburban & Acer negundo L. & 0.0833 & 833 & 200 \\
\hline B11 & Suburban & Acer negundo L. & 0.3333 & 3333 & 67 \\
\hline B 11 & Suburban & Celtis occidentalis L. & 0.3333 & 3333 & 67 \\
\hline B 11 & Suburban & Cornus drummondii C.A. Mey. & 0.3333 & 3333 & 67 \\
\hline $\mathrm{B} 12$ & Suburban & Acer negundo L. & 0.0833 & 1667 & 20 \\
\hline $\mathrm{B} 12$ & Suburban & Acer saccharum Marsh. & 0.1667 & 1667 & 28 \\
\hline B12 & Suburban & Cercis canadensis L. & 0.1667 & 1667 & 28 \\
\hline B12 & Suburban & Celtis occidentalis L. & 0.1667 & 1667 & 28 \\
\hline B 12 & Suburban & Fraxinus americana $\mathrm{L}$. & 0.1667 & 1667 & 28 \\
\hline $\mathrm{B} 12$ & Suburban & Liriodendron tulipifera $\mathrm{L}$. & 0.1667 & 1667 & 28 \\
\hline B12 & Suburban & Platanus occidentalis L. & 0.1667 & 1667 & 28 \\
\hline B 12 & Suburban & Tilia americana $\mathrm{L}$. & 0.0833 & 833 & 14 \\
\hline G1 & Suburban & Acer negundo L. & 0.0833 & 833 & 67 \\
\hline G1 & Suburban & Aesculus glabra Willd. & 0.0833 & 833 & 67 \\
\hline Gl & Suburban & Celtis occidentalis L. & 0.0833 & 833 & 67 \\
\hline G10 & Suburban & Celtis occidentalis L. & 0.1667 & 1667 & 200 \\
\hline G11 & Suburban & Acer negundo L. & 0.3333 & 4167 & 138 \\
\hline G11 & Suburban & Celtis occidentalis L. & 0.1667 & 1667 & 62 \\
\hline G12 & Suburban & Acer negundo L. & 0.0833 & 833 & 19 \\
\hline G12 & Suburban & Acer saccharum Marsh. & 0.1667 & 1667 & 38 \\
\hline G12 & Suburban & Celtis occidentalis L. & 0.3333 & 4167 & 85 \\
\hline G12 & Suburban & Fraxinus americana L. & 0.1667 & 1667 & 38 \\
\hline G12 & Suburban & Liriodendron tulipifera $\mathrm{L}$. & 0.0833 & 833 & 19 \\
\hline G14 & Suburban & Acer negundo $\mathrm{L}$. & 0.3333 & 3333 & 31 \\
\hline G14 & Suburban & Carya cordiformis (Wangenh.) K. Koch & 0.3333 & 3333 & 31 \\
\hline G14 & Suburban & Celtis occidentalis $\mathrm{L}$. & 1.0000 & 23333 & 138 \\
\hline G3 & Suburban & Acer negundo L. & 0.0833 & 833 & 100 \\
\hline G3 & Suburban & Celtis occidentalis $\mathrm{L}$. & 0.0833 & 833 & 100 \\
\hline G6 & Suburban & Acer negundo $\mathrm{L}$. & 0.0833 & 1667 & 16 \\
\hline G6 & Suburban & Acer saccharum Marsh. & 0.2500 & 2500 & 36 \\
\hline G6 & Suburban & Aesculus glabra Willd. & 0.0833 & 833 & 12 \\
\hline G6 & Suburban & Asimina triloba (L.) Dunal & 0.6667 & 14167 & 135 \\
\hline $\mathrm{H} 10$ & Suburban & Fraxinus americana $\mathrm{L}$. & 0.0909 & 909 & 13 \\
\hline $\mathrm{H} 10$ & Suburban & Fraxinus quadrangulata Michx. & 0.2727 & 3636 & 43 \\
\hline $\mathrm{H} 10$ & Suburban & Prunus serotina Ehrh. & 0.4545 & 6364 & 73 \\
\hline $\mathrm{H} 10$ & Suburban & Quercus shumardii Buckl. & 0.0909 & 909 & 13 \\
\hline $\mathrm{H} 10$ & Suburban & Ulmus rubra Muhl. & 0.2727 & 6364 & 58 \\
\hline
\end{tabular}


Appendix Table 10 (cont.)

\begin{tabular}{|c|c|c|}
\hline $\mathrm{H} 2$ & Suburban & Asimina triloba (L.) Dunal \\
\hline $\mathrm{H} 2$ & Suburban & Celtis occidentalis $\mathrm{L}$. \\
\hline $\mathrm{H} 4$ & Suburban & Acer saccharum Marsh. \\
\hline $\mathrm{H} 4$ & Suburban & Aesculus glabra Willd. \\
\hline $\mathrm{H} 4$ & Suburban & Asimina triloba (L.) Dunal \\
\hline $\mathrm{H} 4$ & Suburban & Fraxinus pennsylvanica Marsh. \\
\hline B1 & Urban & Acer negundo L. \\
\hline $\mathrm{B} 13$ & Urban & Fraxinus americana $\mathrm{L}$. \\
\hline $\mathrm{B} 14$ & Urban & Acer negundo L. \\
\hline B 14 & Urban & Aesculus glabra Willd. \\
\hline B 14 & Urban & Platanus occidentalis $\mathrm{L}$. \\
\hline $\mathrm{B} 2$ & Urban & Celtis occidentalis L. \\
\hline B3 & Urban & Celtis occidentalis L. \\
\hline B3 & Urban & Fraxinus sp. \\
\hline B5 & Urban & Acer negundo L. \\
\hline B6 & Urban & Gleditsia tricanthos L. \\
\hline B7 & Urban & Acer negundo L. \\
\hline B7 & Urban & Betula alleghaniensis Britt. \\
\hline B7 & Urban & Celtis occidentalis L. \\
\hline B7 & Urban & Fraxinus americana $\mathrm{L}$. \\
\hline B7 & Urban & Morus rubra $\mathrm{L}$ \\
\hline B8 & Urban & Fraxinus americana $\mathrm{L}$. \\
\hline B8 & Urban & Morus rubra L. \\
\hline $\mathrm{G}$ & Urban & Acer negundo L. \\
\hline G & Urban & Celtis occidentalis L. \\
\hline
\end{tabular}

\begin{tabular}{|c|c|c}
0.1667 & 2500 & 142 \\
0.0833 & 833 & 58 \\
0.1667 & 6667 & 35 \\
0.1667 & 1667 & 20 \\
0.1667 & 5000 & 30 \\
0.6667 & 18333 & 115 \\
0.5833 & 37083 & 200 \\
0.1667 & 1667 & 200 \\
0.2500 & 2500 & 100 \\
0.1667 & 1667 & 67 \\
0.0833 & 833 & 33 \\
0.4167 & 6667 & 200 \\
0.2500 & 4167 & 158 \\
0.0833 & 833 & 42 \\
0.0833 & 833 & 200 \\
0.1667 & 1667 & 200 \\
0.0006 & 556 & 25 \\
0.0003 & 278 & 13 \\
0.0022 & 3333 & 124 \\
0.0006 & 556 & 25 \\
0.0003 & 278 & 13 \\
0.0833 & 833 & 100 \\
0.0833 & 833 & 100 \\
0.3750 & 6250 & 146 \\
0.1250 & 2500 & 54
\end{tabular}


Appendix Table 11: Herbaceous and vine species within 41 riparian sites in Jefferson and Oldham counties in Kentucky. Sites were located in Middle Fork Beargrass, Goose, and South Fork Harrods Creek watersheds. Species codes and wetland indicator status (WIS) were obtained from USDA PLANTS Database (2010).

\begin{tabular}{|c|c|c|}
\hline Species name & Code & WIS \\
\hline Ageratina altissima (L.) King \& H.E. Robins. & AGAL5 & FACU \\
\hline Ageratina spp. L. & AGERA2 & \\
\hline Alliaria petiolata (Bieb.) Cavara \& Grande * & ALPE4 & FACU \\
\hline Allium vineale $\mathrm{L} . *$ & ALVI & FACU \\
\hline Ampelamus albidus (Nutt.) Britt. & AMAL3 & FAC \\
\hline Ambrosia artemisiifolia $\mathrm{L}$. & AMAR2 & FACU \\
\hline Ambrosia trifida $\mathrm{L}$. & AMTR & FAC \\
\hline Arundinaria gigantea (Walt.) Muhl. & ARGI & FACW \\
\hline Aristolochia tomentosa Sims & ARTO3 & $\mathrm{FAC}$ \\
\hline Arisaema triphyllum L. & ARTR & FACW \\
\hline Asarum canadense L. & ASCA & NI \\
\hline Aureolaria spp. L. & AUREO & \\
\hline Bidens spp. L. & BIDEN & FACW \\
\hline Boehmeria cylindrica (L.) Sw. & BOCY & FACW \\
\hline Carex blanda Dewey & CABL & FAC \\
\hline Cardamine pensylvanica Muhl. ex Willd. & CAPE3 & OBL \\
\hline Campsis radicans (L.) Seem. ex Bureau & CARA2 & FAC \\
\hline Cirsium arvense (L.) Scop.* & CIAR4 & FACU \\
\hline Commelina communis L.* & $\mathrm{COCO} 3$ & FAC \\
\hline Cystopteris tenuis (Michx.) Desv. & CYTE7 & $\mathrm{NI}$ \\
\hline Desmodium canescens (L.) DC & DECA8 & NI \\
\hline Desmodium spp. L. & DESMO & \\
\hline Dichanthelium clandestinum (L.) Gould & DICL & $\mathrm{FAC}$ \\
\hline Duchesnea indica (Andr.) Focke* & DUIN & FACU \\
\hline Elephantopus carolinianus Raeusch. & ELCA3 & FACU \\
\hline Elymus riparius Wieg. & ELRI & FACW \\
\hline Elymus spp. L. & ELYMU & \\
\hline Erigeron spp. L. & ERIGE2 & FACU \\
\hline Erigeron philadelphicus L. & ERPH & FACU \\
\hline Euonymus fortunei (Turcz.) Hand.-Maz. * & EUFO5 & $\mathrm{NI}$ \\
\hline Galium aparine L. & GAAP2 & FACU \\
\hline
\end{tabular}


Helianthus spp. L.

HELIA3

Hydrophyllum canadense $\mathrm{L}$.

HYCA3

Impatiens capensis Meerb.

IMCA

FACW

Iodanthus pinnatifidus (Michx.) Steud.

IOPI

Juncus spp. L.

Lamium amplexicaule L.

Lactuca biennis (Moench) Fern.

Lactuca canadensis L.

Lonicera japonica Thunb.*

Lysimachia nummularia L.*

Maianthemum racemosum (L.) Link

Microstegium vimineum (Trin.) A. Camus *

JUNCU

FACW

Ornithogalum umbellatum L.*

LAAM

FACW

Osmorhiza claytonii (Michx.) C.B. Clarke

Oxalis stricta L.

Packera spp. L.

Packera obovata (Muhl. ex Willd.) W.A. Weber \& A. Löve

LABI

NI

LACA

FACU

Parthenocissus quinquefolia (L.) Planch.

Phytolacca americana $\mathrm{L}$.

Pilea pumila (L.) Gray

LOJA

FACU

Plantago rugelii Dcne.

Polygonatum biflorum (Walt.) Ell.

LYNU

FAC

MARA7

OBL

MIVI

FACU

ORUM

FAC

OSCL

FACU

OXST

FACU

PACKE

PAOB6

FACU

PAQU2

FACU

PHAM4

FACU

Polygonum spp. L.

PIPU2

FACW

PLRU

FACU

Podophyllum peltatum $\mathrm{L}$.

Polygonum virginianum $\mathrm{L}$.

Ranunculus hispidus Michx.

Ruellia caroliniensis (J.F. Gmel.) Steud.

POBI2

FACU

POLYG4

POPE

POVI2

FACU

RAHI

FAC

Rudbeckia laciniata $\mathrm{L}$.

Rumex verticillatus $\mathrm{L}$.

Sanicula canadensis $\mathrm{L}$.

Sanicula spp. L.

Sanicula odorata (Raf.) K.M. Pryer \& L.R.

Phillippe

Sedum ternatum Michx.

Securigera varia (L.) Lassen*

Sicyos angulatus L.

\begin{tabular}{l|l} 
RUCA4 & NI \\
RULA3 & FACW \\
RUVE3 & OBL \\
SACA15 & UPL \\
SANIC & \\
& \\
SAOD & FACU \\
SETE3 & NI \\
SEVA4 & NI \\
SIAN & FACU
\end{tabular}


Appendix

Table 11

(cont.)

Smilax rotundifolia $\mathrm{L}$.

Solidago canadensis L.

Sorghum halepense (L.) Pers.*

Solidago spp. L.

Spiraea spp. L.

Stachys spp. L.

Stellaria media (L.) Vill.*

Symphyotrichum cordifolium (L.) Nesom

Symphyotrichum lowrieanum (Porter) Nesom

Symphyotrichum spp. L.

Symphyotrichum pilosum (Willd.) Nesom

Symphyotrichum racemosum (Ell.) Nesom

Taraxacum officinale G.H. Weber ex Wiggers*

Thalictrum thalictroides (L.) Eames \& Boivin

Toxicodendron radicans (L.) Kuntze

Trifolium spp. L.

Trillium spp. L.

Urtica dioica $\mathrm{L}$.

Valerianella umbilicata (Sullivant) Wood

Verbesina alternifolia (L.) Britt. ex Kearney

Veronica arvensis L.*

Vernonia spp. L.

Vinca minor L.*

Viola spp. L.

Viola striata Ait.

Vitis vulpina $\mathrm{L}$.

Woodsia obtusa (Spreng.) Torr.

\begin{tabular}{l|l} 
& \\
SMRO & FAC \\
SOCA6 & FACU \\
SOHA & FACU \\
SOLID & \\
SPIRA & \\
STACH & \\
STME2 & UPL \\
SYCO4 & NI \\
SYLO2 & NI \\
SYMPH4 & \\
SYPI2 & UPL \\
SYRA5 & FACW \\
TAOF & FACU \\
THTH2 & NI \\
TORA2 & FAC \\
TRIFO & \\
TRILL & \\
URDI & FACU \\
VAUM & FAC \\
VEAL & FAC \\
VEAR & NI \\
VERNO & \\
VIMI2 & NI \\
VIOLA & \\
VIST3 & FACW \\
VIVU & FAC \\
WOOB2 & NI \\
\hline
\end{tabular}

Non-native species indicated with an $\left(^{*}\right)$

Note: Plants were identified only to genus group if species could not be determined 
Appendix Table 12: Herbaceous and vine species within 41 riparian sites in Jefferson and Oldham counties in Kentucky. Site names refer to the watershed in which the site was located, where $\mathrm{B}=$ Middle Fork Beargrass, $\mathrm{G}=$ Goose, and $\mathrm{H}=$ South Fork Harrods Creek watersheds. Importance values (IV) for each species were calculated by adding average absolute \% cover and frequency for each site. Data within mult-plot sites were combined. Land use categories for each site were determined by the proportion of impervious surface (IS) within the subcatchment in which the site was located. Urban $\geq 30 \%$ IS, Rural $\leq 10 \%$ IS, and Suburban is between 10 and $30 \%$ IS.

\begin{tabular}{|c|c|c|c|c|c|}
\hline Site & $\begin{array}{l}\text { Land use } \\
\text { categ. }\end{array}$ & Species name & $\begin{array}{l}\text { Avg. } \\
\% \\
\text { cover }\end{array}$ & Freq & IV \\
\hline G2 & Rural & $\begin{array}{l}\text { Ageratina altissima (L.) King \& H.E. } \\
\text { Robins. }\end{array}$ & 0.96 & 17 & 18 \\
\hline G2 & Rural & Duchesnea indica (Andr.) Focke* & 0.58 & 6 & 6 \\
\hline G2 & Rural & Euonymus fortunei (Turcz.) Hand.-Maz. * & 12.83 & 61 & 73 \\
\hline $\mathrm{G} 2$ & Rural & Galium aparine $\mathrm{L}$. & 0.42 & 11 & 11 \\
\hline $\mathrm{G} 2$ & Rural & Glechoma hederacea L.* & 0.67 & 44 & 45 \\
\hline $\mathrm{G} 2$ & Rural & Impatiens capensis Meerb. & 0.04 & 6 & 6 \\
\hline $\mathrm{G} 2$ & Rural & Oxalis stricta $\mathrm{L}$. & 0.50 & 6 & 6 \\
\hline G2 & Rural & Plantago rugelii Dcne. & 0.83 & 17 & 18 \\
\hline $\mathrm{G} 2$ & Rural & $\begin{array}{l}\text { Sanicula odorata (Raf.) K.M. Pryer \& L.R. } \\
\text { Phillippe }\end{array}$ & 0.58 & 17 & 18 \\
\hline G2 & Rural & $\begin{array}{l}\text { Taraxacum officinale G.H. Weber ex } \\
\text { Wiggers* }\end{array}$ & 0.17 & 6 & 6 \\
\hline G2 & Rural & Toxicodendron radicans (L.) Kuntze & 0.67 & 17 & 18 \\
\hline $\mathrm{G} 2$ & Rural & Veronica arvensis L.* & 1.50 & 22 & 23 \\
\hline $\mathrm{G} 2$ & Rural & Vinca minor L.* & 12.96 & 44 & 56 \\
\hline $\mathrm{G} 2$ & Rural & Viola spp. L. & 0.25 & 28 & 28 \\
\hline G4 & Rural & $\begin{array}{l}\text { Ageratina altissima (L.) King \& H.E. } \\
\text { Robins. }\end{array}$ & 32.89 & 100 & 133 \\
\hline $\mathrm{G} 4$ & Rural & Duchesnea indica (Andr.) Focke* & 0.78 & 22 & 23 \\
\hline G4 & Rural & Juncus spp. L. & 2.78 & 56 & 58 \\
\hline $\mathrm{G} 4$ & Rural & Lonicera japonica Thunb.* & 1.33 & 22 & 24 \\
\hline G4 & Rural & Microstegium vimineum (Trin.) A. Camus * & 0.11 & 11 & 11 \\
\hline G4 & Rural & Parthenocissus quinquefolia (L.) Planch. & 3.94 & 78 & 82 \\
\hline G4 & Rural & $\begin{array}{l}\text { Sanicula odorata (Raf.) K.M. Pryer \& L.R. } \\
\text { Phillippe }\end{array}$ & 1.61 & 56 & 57 \\
\hline G4 & Rural & Solidago spp. L. & 1.28 & 44 & 46 \\
\hline G4 & Rural & Symphyotrichum cordifolium (L.) Nesom & 0.22 & 11 & 11 \\
\hline G4 & Rural & Toxicodendron radicans (L.) Kuntze & 0.06 & 11 & 11 \\
\hline
\end{tabular}




\begin{tabular}{|c|c|c|c|c|c|}
\hline \multicolumn{6}{|c|}{ Appendix Table 12 (cont.) } \\
\hline G4 & Rural & Verbesina alternifolia (L.) Britt. ex Kearney & 4.44 & 11 & 16 \\
\hline G7 & Rural & $\begin{array}{l}\text { Ageratina altissima (L.) King \& H.E. } \\
\text { Robins. }\end{array}$ & 12.75 & 100 & 113 \\
\hline G7 & Rural & Asarum canadense $\mathrm{L}$. & 0.08 & 17 & 17 \\
\hline G7 & Rural & Glechoma hederacea L.* & 2.75 & 100 & 103 \\
\hline G7 & Rural & Grass & 0.83 & 17 & 18 \\
\hline G7 & Rural & Microstegium vimineum (Trin.) A. Camus * & 1.83 & 33 & 35 \\
\hline G7 & Rural & $\begin{array}{l}\text { Sanicula odorata (Raf.) K.M. Pryer \& L.R. } \\
\text { Phillippe }\end{array}$ & 0.33 & 17 & 17 \\
\hline G7 & Rural & Symphyotrichum spp. L. & 3.33 & 67 & 70 \\
\hline G7 & Rural & Verbesina alternifolia (L.) Britt. ex Kearney & 0.50 & 17 & 17 \\
\hline $\mathrm{H} 1$ & Rural & $\begin{array}{l}\text { Ageratina altissima (L.) King \& H.E. } \\
\text { Robins. }\end{array}$ & 0.83 & 25 & 26 \\
\hline $\mathrm{H} 1$ & Rural & Alliaria petiolata (Bieb.) Cavara \& Grande * & 4.33 & 33 & 38 \\
\hline $\mathrm{Hl}$ & Rural & Allium vineale L.* & 0.17 & 33 & 34 \\
\hline $\mathrm{H} 1$ & Rural & Ambrosia artemisiifolia $\mathrm{L}$. & 0.04 & 8 & 8 \\
\hline $\mathrm{H} 1$ & Rural & Arisaema triphyllum L. & 0.04 & 8 & 8 \\
\hline $\mathrm{H} 1$ & Rural & Apiaceae & 0.04 & 8 & 8 \\
\hline $\mathrm{H} 1$ & Rural & Desmodium spp. L. & 0.13 & 17 & 17 \\
\hline $\mathrm{H} 1$ & Rural & Duchesnea indica (Andr.) Focke* & 1.29 & 67 & 68 \\
\hline H1 & Rural & Elymus riparius Wieg. & 0.46 & 33 & 34 \\
\hline $\mathrm{H} 1$ & Rural & Erigeron philadelphicus L. & 0.04 & 8 & 8 \\
\hline $\mathrm{H} 1$ & Rural & Galium aparine $\mathrm{L}$. & 1.71 & 50 & 52 \\
\hline H1 & Rural & Glechoma hederacea L.* & 8.42 & 100 & 108 \\
\hline $\mathrm{H} 1$ & Rural & Impatiens capensis Meerb. & 0.17 & 17 & 17 \\
\hline $\mathrm{H} 1$ & Rural & Lonicera japonica Thunb.* & 0.58 & 67 & 67 \\
\hline $\mathrm{HI}$ & Rural & Lamiaceae & 0.04 & 8 & 8 \\
\hline $\mathrm{H} 1$ & Rural & Ornithogalum umbellatum L.* & 0.04 & 8 & 8 \\
\hline $\mathrm{H} 1$ & Rural & Osmorhiza claytonii (Michx.) C.B. Clarke & 1.38 & 50 & 51 \\
\hline $\mathrm{H} 1$ & Rural & Packera spp. L. & 0.04 & 8 & 8 \\
\hline $\mathrm{H} 1$ & Rural & Parthenocissus quinquefolia (L.) Planch. & 0.42 & 25 & 25 \\
\hline $\mathrm{H} 1$ & Rural & Pilea pumila (L.) Gray & 0.33 & 42 & 42 \\
\hline $\mathrm{H} 1$ & Rural & Podophyllum peltatum $\mathrm{L}$. & 1.08 & 8 & 9 \\
\hline $\mathrm{H} 1$ & Rural & Sanicula spp. L. & 1.50 & 58 & 60 \\
\hline $\mathrm{H} 1$ & Rural & $\begin{array}{l}\text { Sanicula odorata (Raf.) K.M. Pryer \& L.R. } \\
\text { Phillippe }\end{array}$ & 7.00 & 100 & 107 \\
\hline $\mathrm{H} 1$ & Rural & Smilax rotundifolia $\mathrm{L}$. & 0.42 & 25 & 25 \\
\hline $\mathrm{H} 1$ & Rural & Solidago spp. L. & 0.58 & 25 & 26 \\
\hline $\mathrm{H} 1$ & Rural & Stachys spp. L. & 0.08 & 8 & 8 \\
\hline $\mathrm{H} 1$ & Rural & $\begin{array}{l}\text { Thalictrum thalictroides (L.) Eames \& } \\
\text { Boivin }\end{array}$ & 0.13 & 17 & 17 \\
\hline
\end{tabular}




\begin{tabular}{|c|c|c|c|c|c|}
\hline & & Appendix Table 12 (cont.) & & & \\
\hline $\mathrm{H} 1$ & Rural & Toxicodendron radicans (L.) Kuntze & 0.33 & 17 & 17 \\
\hline $\mathrm{H} 1$ & Rural & Trillium spp. L. & 0.13 & 17 & 17 \\
\hline $\mathrm{H} 1$ & Rural & Urtica dioica $\mathrm{L}$. & 0.17 & 8 & 9 \\
\hline $\mathrm{H} 1$ & Rural & Verbesina alternifolia (L.) Britt. ex Kearney & 0.17 & 8 & 9 \\
\hline $\mathrm{H} 1$ & Rural & Viola spp. L. & 0.38 & 42 & 42 \\
\hline $\mathrm{H} 1$ & Rural & Viola striata Ait. & 0.75 & 42 & 42 \\
\hline $\mathrm{H} 1$ & Rural & Vitis vulpina $\mathrm{L}$. & 0.08 & 8 & 8 \\
\hline $\mathrm{H} 11$ & Rural & $\begin{array}{l}\text { Ageratina altissima (L.) King \& H.E. } \\
\text { Robins. }\end{array}$ & 0.21 & 8 & 9 \\
\hline H11 & Rural & Glechoma hederacea L.* & 8.00 & 67 & 75 \\
\hline H11 & Rural & Lonicera japonica Thunb.* & 0.67 & 42 & 42 \\
\hline $\mathrm{H} 11$ & Rural & Lamiaceae & 0.29 & 17 & 17 \\
\hline H11 & Rural & Microstegium vimineum (Trin.) A. Camus * & 10.00 & 33 & 43 \\
\hline H11 & Rural & $\begin{array}{l}\text { Sanicula odorata (Raf.) K.M. Pryer \& L.R. } \\
\text { Phillippe }\end{array}$ & 0.75 & 17 & 17 \\
\hline H11 & Rural & Solidago spp. L. & 0.50 & 25 & 26 \\
\hline H11 & Rural & Symphyotrichum lowrieanum (Porter) Nesom & 0.25 & 8 & 9 \\
\hline H11 & Rural & Toxicodendron radicans (L.) Kuntze & 0.58 & 17 & 17 \\
\hline H11 & Rural & Viola striata Ait. & 0.04 & 8 & 8 \\
\hline $\mathrm{H} 12$ & Rural & $\begin{array}{l}\text { Ageratina altissima (L.) King \& H.E. } \\
\text { Robins. }\end{array}$ & 4.43 & 63 & 67 \\
\hline $\mathrm{H} 12$ & Rural & Ambrosia trifida $\mathrm{L}$. & 0.19 & 8 & 9 \\
\hline $\mathrm{H} 12$ & Rural & Cirsium arvense (L.) Scop.* & 0.04 & 4 & 4 \\
\hline $\mathrm{H} 12$ & Rural & Desmodium spp. L. & 0.06 & 4 & 4 \\
\hline $\mathrm{H} 12$ & Rural & Elephantopus carolinianus Raeusch. & 0.13 & 13 & 13 \\
\hline $\mathrm{H} 12$ & Rural & Glechoma hederacea L.* & 0.13 & 4 & 4 \\
\hline $\mathrm{H} 12$ & Rural & Lactuca biennis (Moench) Fern. & 0.42 & 21 & 21 \\
\hline $\mathrm{H} 12$ & Rural & Lonicera japonica Thunb.* & 2.32 & 42 & 44 \\
\hline $\mathrm{H} 12$ & Rural & Lysimachia nummularia L.* & 1.69 & 33 & 35 \\
\hline $\mathrm{H} 12$ & Rural & Microstegium vimineum (Trin.) A. Camus * & 8.63 & 29 & 38 \\
\hline $\mathrm{H} 12$ & Rural & Packera spp. L. & 0.08 & 8 & 8 \\
\hline $\mathrm{H} 12$ & Rural & $\begin{array}{l}\text { Sanicula odorata (Raf.) K.M. Pryer \& L.R. } \\
\text { Phillippe }\end{array}$ & 2.69 & 42 & 44 \\
\hline $\mathrm{H} 12$ & Rural & Securigera varia (L.) Lassen* & 2.42 & 21 & 23 \\
\hline $\mathrm{H} 12$ & Rural & Solidago canadensis L. & 0.92 & 17 & 17 \\
\hline $\mathrm{H} 12$ & Rural & Solidago spp. L. & 0.31 & 12 & 13 \\
\hline $\mathrm{H} 12$ & Rural & Symphyotrichum pilosum (Willd.) Nesom & 0.83 & 8 & 9 \\
\hline $\mathrm{H} 12$ & Rural & Toxicodendron radicans (L.) Kuntze & 0.29 & 12 & 13 \\
\hline $\mathrm{H} 12$ & Rural & Trifolium spp. L. & 0.21 & 4 & 4 \\
\hline $\mathrm{H} 12$ & Rural & Verbesina alternifolia (L.) Britt. ex Kearney & 1.56 & 42 & 43 \\
\hline $\mathrm{H} 12$ & Rural & Viola spp. L. & 0.13 & 8 & 8 \\
\hline
\end{tabular}




\begin{tabular}{l|l} 
H13 & Rural \\
H13 & Rural \\
H14 & Rural \\
H14 & Rural \\
H14 & Rural \\
H14 & Rural \\
H14 & Rural \\
H14 & Rural \\
H14 & Rural \\
H14 & Rural \\
H14 & Rural \\
H14 & Rural \\
H14 & Rural \\
H14 & Rural \\
H14 & Rural \\
H14 & Rural \\
H14 & Rural \\
H14 & Rural \\
H3 & Rural \\
H14 & Rural \\
H14 & Rural \\
H14 & Rural \\
H14 & Rural \\
H3 & Rural \\
H3 & Rural \\
H3 & Rural \\
H3 & Rural \\
H3 & Rural \\
H3 & Rural \\
H3 & Rural \\
H3 & Rural \\
H3 & Rural \\
H3
\end{tabular}

\section{Appendix Table 12 (cont.)}

Securigera varia (L.) Lassen*

Vernonia spp. L.

Ageratina altissima (L.) King \& H.E.

Robins.

Alliaria petiolata (Bieb.) Cavara \& Grande *

Asarum canadense L.

Desmodium canescens (L.) DC

Elymus spp. L.

Euonymus fortunei (Turcz.) Hand.-Maz. *

Glechoma hederacea L.*

Grass

Hydrophyllum canadense L.

Microstegium vimineum (Trin.) A. Camus *

Phytolacca americana L.

Polygonum spp. L.

Polygonum virginianum $\mathrm{L}$.

Rudbeckia laciniata L.

Sanicula odorata (Raf.) K.M. Pryer \& L.R.

Phillippe

Symphyotrichum spp. L.

Urtica dioica L.

Verbesina alternifolia (L.) Britt. ex Kearney

Viola striata Ait.

Woodsia obtusa (Spreng.) Torr.

Ageratina altissima (L.) King \& H.E.

Robins.

Boehmeria cylindrica (L.) Sw.

Apiaceae

Duchesnea indica (Andr.) Focke*

Euonymus fortunei (Turcz.) Hand.-Maz. *

Glechoma hederacea L.*

Grass

Lysimachia nummularia L.*

Microstegium vimineum (Trin.) A. Camus *

Sanicula odorata (Raf.) K.M. Pryer \& L.R.

Phillippe

Spiraea spp. L.

Symphyotrichum spp. L.

Symphyotrichum racemosum (E11.) Nesom

Viola spp. L.

\begin{tabular}{|l|l|l}
4.75 & 67 & 71 \\
0.08 & 17 & 17 \\
0.21 & 8 & 9 \\
0.33 & 21 & 21 \\
0.15 & 13 & 13 \\
9.50 & 54 & 64 \\
0.08 & 4 & 4 \\
0.08 & 8 & 8 \\
20.94 & 87 & 108 \\
2.46 & 17 & 19 \\
5.20 & 88 & 93 \\
0.04 & 4 & 4 \\
0.29 & 4 & 4 \\
1.54 & 13 & 14 \\
0.46 & 4 & 5 \\
0.54 & 8 & 9 \\
0.23 & 21 & 21 \\
0.92 & 29 & 30 \\
20.28 & 58 & 78 \\
1.44 & 21 & 22 \\
0.46 & 21 & 21 \\
0.02 & 4 & 4 \\
0.83 & 17 & 18 \\
0.92 & 8 & 9 \\
0.17 & 25 & 25 \\
0.96 & 25 & 26 \\
0.17 & 25 & 25 \\
1.00 & 33 & 34 \\
13.75 & 33 & 47 \\
0.04 & 8 & 8 \\
7.50 & 42 & 49 \\
0.25 & 17 & 17 \\
0.33 & 8 & 9 \\
0.96 & 33 & 34 \\
0.17 & 8 & 9 \\
1.21 & 42 & 43 \\
& & \\
0.21 \\
0.08
\end{tabular}


Appendix Table 12 (cont.)

\begin{tabular}{|c|c|c|c|c|}
\hline $\mathrm{H} 5$ & Rural & $\begin{array}{l}\text { Ageratina altissima (L.) King \& H.E. } \\
\text { Robins. }\end{array}$ & 2.10 & 71 \\
\hline $\mathrm{H} 5$ & Rural & Alliaria petiolata (Bieb.) Cavara \& Grande * & 0.06 & 13 \\
\hline $\mathrm{H} 5$ & Rural & Allium vineale L.* & 0.27 & 54 \\
\hline H5 & Rural & Arisaema triphyllum $\mathrm{L}$. & 0.08 & 4 \\
\hline $\mathrm{H} 5$ & Rural & Asarum canadense L. & 0.10 & 13 \\
\hline $\mathrm{H} 5$ & Rural & Carex blanda Dewey & 0.27 & 25 \\
\hline $\mathrm{H} 5$ & Rural & Cystopteris tenuis (Michx.) Desv. & 0.02 & 4 \\
\hline H5 & Rural & Desmodium spp. L. & 0.38 & 33 \\
\hline $\mathrm{H} 5$ & Rural & Dichanthelium clandestinum (L.) Gould & 0.17 & 8 \\
\hline $\mathrm{H} 5$ & Rural & Elephantopus carolinianus Raeusch. & 0.29 & 25 \\
\hline $\mathrm{H} 5$ & Rural & Erigeron philadelphicus L. & 0.08 & 13 \\
\hline H5 & Rural & Galium aparine $\mathrm{L}$. & 0.15 & 17 \\
\hline H5 & Rural & Glechoma hederacea L.* & 5.71 & 79 \\
\hline $\mathrm{H} 5$ & Rural & Grass & 1.77 & 54 \\
\hline $\mathrm{H} 5$ & Rural & Impatiens capensis Meerb. & 0.04 & 8 \\
\hline $\mathrm{H} 5$ & Rural & Iodanthus pinnatifidus (Michx.) Steud. & 0.06 & 8 \\
\hline H5 & Rural & Lonicera japonica Thunb.* & 1.10 & 29 \\
\hline $\mathrm{H} 5$ & Rural & Lysimachia nummularia L.* & 0.06 & 8 \\
\hline H5 & Rural & Lamiaceae & 0.08 & 4 \\
\hline $\mathrm{H} 5$ & Rural & Microstegium vimineum (Trin.) A. Camus * & 5.52 & 50 \\
\hline H5 & Rural & Packera spp. L. & 0.02 & 4 \\
\hline H5 & Rural & Pilea pumila (L.) Gray & 0.23 & 37 \\
\hline H5 & Rural & Plantago rugelii Dcne. & 0.02 & 4 \\
\hline H5 & Rural & Polygonum spp. L. & 0.42 & 4 \\
\hline H5 & Rural & Sanicula canadensis L. & 2.38 & 71 \\
\hline H5 & Rural & $\begin{array}{l}\text { Sanicula odorata (Raf.) K.M. Pryer \& L.R. } \\
\text { Phillippe }\end{array}$ & 0.56 & 50 \\
\hline $\mathrm{H} 5$ & Rural & Solidago spp. L. & 0.31 & 29 \\
\hline H5 & Rural & Symphyotrichum cordifolium (L.) Nesom & 0.06 & 13 \\
\hline H5 & Rural & $\begin{array}{l}\text { Thalictrum thalictroides (L.) Eames \& } \\
\text { Boivin }\end{array}$ & 0.04 & 8 \\
\hline I5 & Rural & Toxicodendron radicans (L.) Kuntze & 0.02 & 4 \\
\hline H5 & Rural & Verbesina alternifolia (L.) Britt. ex Kearney & 1.00 & 37 \\
\hline $\mathrm{H} 5$ & Rural & Viola spp. L. & 0.79 & 59 \\
\hline H5 & Rural & Viola striata Ait. & 0.33 & 38 \\
\hline H6 & Rural & $\begin{array}{l}\text { Ageratina altissima (L.) King \& H.E. } \\
\text { Robins. }\end{array}$ & 1.11 & 89 \\
\hline $\mathrm{H}$ & Rural & Asarum canadense L. & 0.72 & 67 \\
\hline $\mathrm{He}$ & Rural & Grass & 0.28 & 44 \\
\hline $\mathrm{H} 6$ & Rural & Lonicera japonica Thunb.* & 0.39 & 67 \\
\hline
\end{tabular}




\begin{tabular}{|c|c|c|c|c|c|}
\hline & & Appendix Table 12 (cont.) & & & \\
\hline H6 & Rural & Lysimachia nummularia L.* & 0.28 & 44 & 45 \\
\hline H6 & Rural & $\begin{array}{l}\text { Packera obovata (Muhl. ex Willd.) W.A. } \\
\text { Weber \& A. Löve }\end{array}$ & 0.11 & 22 & 22 \\
\hline H6 & Rural & Ruellia caroliniensis (J.F. Gmel.) Steud. & 0.11 & 11 & 11 \\
\hline H6 & Rural & $\begin{array}{l}\text { Sanicula odorata (Raf.) K.M. Pryer \& L.R. } \\
\text { Phillippe }\end{array}$ & 0.17 & 33 & 34 \\
\hline H6 & Rural & Symphyotrichum cordifolium (L.) Nesom & 0.22 & 11 & 11 \\
\hline I6 & Rural & Viola spp. L. & 0.61 & 67 & 67 \\
\hline $\mathrm{H} 6$ & Rural & Viola striata Ait. & 0.11 & 17 & 17 \\
\hline $\mathrm{H} 7$ & Rural & $\begin{array}{l}\text { Ageratina altissima (L.) King \& H.E. } \\
\text { Robins. }\end{array}$ & 0.94 & 50 & 51 \\
\hline $\mathrm{H} 7$ & Rural & Asarum canadense L. & 4.63 & 100 & 105 \\
\hline $\mathrm{H} 7$ & Rural & Asteraceae & 0.69 & 75 & 76 \\
\hline $\mathrm{H} 7$ & Rural & Aureolaria spp. L. & 0.25 & 38 & 38 \\
\hline $\mathrm{H} 7$ & Rural & Lysimachia nummularia L.* & 0.31 & 50 & 50 \\
\hline $\mathrm{H} 7$ & Rural & Ranunculus hispidus Michx. & 0.19 & 13 & 13 \\
\hline H7 & Rural & $\begin{array}{l}\text { Sanicula odorata (Raf.) K.M. Pryer \& L.R. } \\
\text { Phillippe }\end{array}$ & 0.19 & 38 & 38 \\
\hline $\mathrm{H} 7$ & Rural & Symphyotrichum lowrieanum (Porter) Nesom & 0.13 & 13 & 13 \\
\hline $\mathrm{H} 7$ & Rural & Viola spp. L. & 2.81 & 100 & 103 \\
\hline $\mathrm{H} 8$ & Rural & $\begin{array}{l}\text { Ageratina altissima (L.) King \& H.E. } \\
\text { Robins. }\end{array}$ & 0.25 & 25 & 25 \\
\hline $\mathrm{H} 8$ & Rural & Allium vineale $\mathrm{L} . *$ & 0.31 & 63 & 63 \\
\hline $\mathrm{H} 8$ & Rural & Arisaema triphyllum L. & 0.63 & 4 & 5 \\
\hline $\mathrm{H} 8$ & Rural & Asarum canadense L. & 0.21 & 17 & 17 \\
\hline $\mathrm{H} 8$ & Rural & Aureolaria spp. L. & 0.06 & 8 & 8 \\
\hline H8 & Rural & Carex blanda Dewey & 0.02 & 4 & 4 \\
\hline $\mathrm{H} 8$ & Rural & Cystopteris tenuis (Michx.) Desv. & 0.06 & 17 & 17 \\
\hline $\mathrm{H} 8$ & Rural & Desmodium spp. L. & 0.21 & 29 & 29 \\
\hline $\mathrm{H} 8$ & Rural & Duchesnea indica (Andr.) Focke* & 0.02 & 4 & 4 \\
\hline $\mathrm{H} 8$ & Rural & Elymus riparius Wieg. & 0.04 & 4 & 4 \\
\hline $\mathrm{H} 8$ & Rural & Erigeron philadelphicus L. & 0.02 & 4 & 4 \\
\hline $\mathrm{H} 8$ & Rural & Galium aparine $\mathrm{L}$. & 0.13 & 25 & 25 \\
\hline $\mathrm{H} 8$ & Rural & Geum vernum (Raf.) Torr. \& Gray & 0.08 & 13 & 13 \\
\hline $\mathrm{H} 8$ & Rural & Grass & 0.81 & 29 & 30 \\
\hline $\mathrm{H} 8$ & Rural & Impatiens capensis Meerb. & 0.04 & 8 & 8 \\
\hline $\mathrm{H} 8$ & Rural & Iodanthus pinnatifidus (Michx.) Steud. & 0.02 & 4 & 4 \\
\hline $\mathrm{H} 8$ & Rural & Juncus spp. L. & 0.21 & 21 & 21 \\
\hline $\mathrm{H} 8$ & Rural & Lonicera japonica Thunb.* & 0.02 & 4 & 4 \\
\hline H8 & Rural & Lysimachia nummularia L.* & 2.60 & 59 & 61 \\
\hline H8 & Rural & Microstegium vimineum (Trin.) A. Camus * & 1.50 & 13 & 14 \\
\hline
\end{tabular}




\section{Appendix Table 12 (cont.)}

\begin{tabular}{|c|c|c|c|c|c|}
\hline H8 & Rural & Packera spp. L. & 0.21 & 29 & 30 \\
\hline $\mathrm{H} 8$ & Rural & $\begin{array}{l}\text { Packera obovata (Muhl. ex Willd.) W.A. } \\
\text { Weber \& A. Löve }\end{array}$ & 0.02 & 4 & 4 \\
\hline $\mathrm{H} 8$ & Rural & Pilea pumila (L.) Gray & 0.70 & 62 & 63 \\
\hline $\mathrm{H} 8$ & Rural & Plantago rugelii Dene. & 0.04 & 8 & \\
\hline $\mathrm{H} 8$ & Rural & Ranunculus hispidus Michx. & 0.04 & 8 & 8 \\
\hline H8 & Rural & Sanicula canadensis $\mathrm{L}$. & 2.83 & 58 & 61 \\
\hline H8 & Rural & Sanicula spp. L. & 0.17 & 25 & 5 \\
\hline H8 & Rural & $\begin{array}{l}\text { Sanicula odorata (Raf.) K.M. Pryer \& L.R. } \\
\text { Phillippe }\end{array}$ & 2.42 & 71 & 73 \\
\hline $\mathrm{H} 8$ & Rural & Sedum ternatum Michx. & 0.15 & 8 & 8 \\
\hline $\mathrm{H} 8$ & Rural & Smilax rotundifolia $\mathrm{L}$. & 0.02 & 4 & 4 \\
\hline $\mathrm{H} 8$ & Rural & Solidago spp. L. & 0.50 & 38 & 38 \\
\hline $\mathrm{H} 8$ & Rural & Symphyotrichum cordifolium (L.) Nesom & 0.10 & 17 & 17 \\
\hline H8 & Rural & $\begin{array}{l}\text { Thalictrum thalictroides (L.) Eames \& } \\
\text { Boivin }\end{array}$ & 0.06 & 8 & 8 \\
\hline H8 & Rural & Valerianella umbilicata (Sullivant) Wood & 0.06 & 8 & 8 \\
\hline $\mathrm{H} 8$ & Rural & Verbesina alternifolia (L.) Britt. ex Kearney & 1.08 & 42 & 43 \\
\hline $\mathrm{H} 8$ & Rural & Viola spp. L. & 1.48 & 83 & 85 \\
\hline H8 & Rural & Viola striata Ait. & 0.48 & 54 & 55 \\
\hline H9 & Rural & Campsis radicans (L.) Seem. ex Bureau & 0.83 & 17 & 18 \\
\hline H9 & Rural & Lonicera japonica Thunb.* & 0.67 & 33 & 34 \\
\hline H9 & Rural & Lysimachia nummularia $\mathrm{L} . *$ & 0.33 & 17 & 17 \\
\hline $\mathrm{H} 9$ & Rural & Microstegium vimineum (Trin.) A. Camus * & 0.08 & 17 & 17 \\
\hline H9 & Rural & Packera spp. L. & 0.25 & 33 & 34 \\
\hline H9 & Rural & Plantago rugelii Dcne. & 0.17 & 17 & 17 \\
\hline H9 & Rural & $\begin{array}{l}\text { Sanicula odorata (Raf.) K.M. Pryer \& L.R. } \\
\text { Phillippe }\end{array}$ & 5.33 & 83 & 39 \\
\hline H9 & Rural & Solidago spp. L. & 0.42 & 33 & 34 \\
\hline H9 & Rural & Toxicodendron radicans (L.) Kuntze & 0.33 & 33 & 34 \\
\hline H9 & Rural & Viola spp. L. & 0.50 & 17 & 17 \\
\hline H9 & Rural & Vitis vulpina $\mathrm{L}$. & 0.33 & 33 & 34 \\
\hline B10 & Suburban & $\begin{array}{l}\text { Ageratina altissima (L.) King \& H.E. } \\
\text { Robins. }\end{array}$ & 0.08 & 8 & 8 \\
\hline B10 & Suburban & Ambrosia trifida $\mathrm{L}$. & 6.92 & 17 & 24 \\
\hline B10 & Suburban & Bidens spp. L. & 0.58 & 8 & 9 \\
\hline $\mathrm{B} 10$ & Suburban & Commelina communis L.* & 0.17 & 8 & 9 \\
\hline $\mathrm{B} 10$ & Suburban & Duchesnea indica (Andr.) Focke* & 0.08 & 8 & 8 \\
\hline $\mathrm{B} 10$ & Suburban & Elephantopus carolinianus Raeusch. & 0.67 & 17 & 17 \\
\hline $\mathrm{B} 10$ & Suburban & Euonymus fortunei (Turcz.) Hand.-Maz. * & 4.17 & 8 & 13 \\
\hline B10 & Suburban & Glechoma hederacea L.* & 0.42 & 8 & 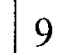 \\
\hline
\end{tabular}




\section{Appendix Table 12 (cont.)}

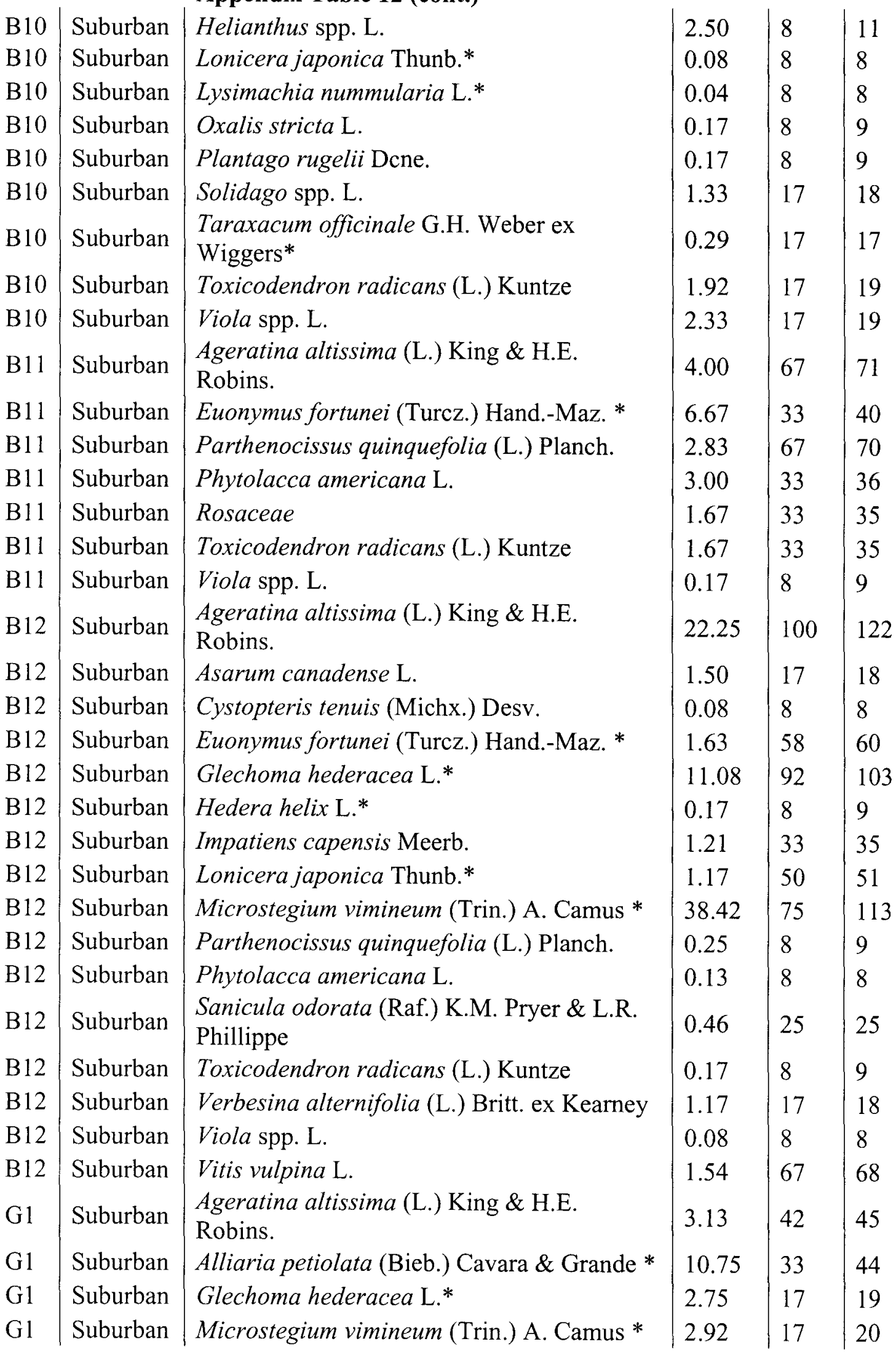




\section{Appendix Table 12 (cont.)}

\begin{tabular}{|c|c|c|c|c|c|}
\hline G1 & Suburban & Stellaria media (L.) Vill.* & 0.08 & 8 & 8 \\
\hline $\mathrm{j} 10$ & Suburban & $\begin{array}{l}\text { Ageratina altissima (L.) King \& H.E. } \\
\text { Robins. }\end{array}$ & 2.42 & 42 & 44 \\
\hline 10 & Suburban & Alliaria petiolata (Bieb.) Cavara \& Grande * & 0.67 & 8 & 9 \\
\hline 10 & Suburban & Desmodium spp. L. & 1.00 & 50 & 51 \\
\hline 10 & Suburban & Elymus riparius Wieg. & 3.83 & 83 & 87 \\
\hline G10 & Suburban & Euonymus fortunei (Turcz.) Hand.-Maz. * & 0.92 & 8 & 9 \\
\hline G10 & Suburban & Galium aparine L. & 0.04 & 8 & 8 \\
\hline G10 & Suburban & Glechoma hederacea L.* & 18.25 & 100 & 118 \\
\hline 10 & Suburban & Impatiens capensis Meerb. & 0.17 & 25 & 25 \\
\hline 10 & Suburban & Iodanthus pinnatifidus (Michx.) Steud. & 0.17 & 8 & 9 \\
\hline 10 & Suburban & Parthenocissus quinquefolia (L.) Planch. & 0.25 & 17 & 17 \\
\hline 10 & Suburban & Rudbeckia laciniata L. & 2.58 & 33 & 36 \\
\hline G10 & Suburban & $\begin{array}{l}\text { Sanicula odorata (Raf.) K.M. Pryer \& L.R. } \\
\text { Phillippe }\end{array}$ & 0.83 & 25 & 26 \\
\hline 10 & Suburban & Smilax rotundifolia $\mathrm{L}$. & 1.25 & 8 & 10 \\
\hline G10 & Suburban & Solidago spp. L. & 8.75 & 92 & 100 \\
\hline G10 & Suburban & Verbesina alternifolia (L.) Britt. ex Kearney & 10.08 & 67 & 77 \\
\hline G10 & Suburban & Viola spp. L. & 0.08 & 8 & 8 \\
\hline G11 & Suburban & $\begin{array}{l}\text { Ageratina altissima (L.) King \& H.E. } \\
\text { Robins. }\end{array}$ & 2.33 & 42 & 4 \\
\hline G11 & Suburban & Allium vineale $\mathrm{L} . *$ & 0.04 & 8 & 8 \\
\hline G11 & Suburban & Desmodium spp. L. & 0.58 & 67 & 67 \\
\hline G11 & Suburban & Duchesnea indica (Andr.) Focke* & 0.71 & 33 & 34 \\
\hline G11 & Suburban & Euonymus fortunei (Turcz.) Hand.-Maz. * & 28.42 & 67 & 95 \\
\hline G11 & Suburban & Glechoma hederacea L.* & 9.42 & 50 & 59 \\
\hline G11 & Suburban & Grass & 10.83 & 25 & 36 \\
\hline G11 & Suburban & Impatiens capensis Meerb. & 1.00 & 8 & 9 \\
\hline G11 & Suburban & Lonicera japonica Thunb.* & 1.83 & 50 & 52 \\
\hline G11 & Suburban & Lysimachia nummularia L.* & 0.42 & 25 & 25 \\
\hline G1 & Suburban & Microstegium vimineum (Trin.) A. Camus * & 7.83 & 17 & 25 \\
\hline G1 & Suburban & Plantago rugelii Dene. & 0.08 & 8 & 8 \\
\hline G11 & Suburban & $\begin{array}{l}\text { Sanicula odorata (Raf.) K.M. Pryer \& L.R. } \\
\text { Phillippe }\end{array}$ & 0.54 & 25 & 26 \\
\hline G & Suburban & Smilax rotundifolia $\mathrm{L}$. & 0.04 & 8 & 8 \\
\hline G1 & Suburban & Sorghum halepense (L.) Pers.* & 0.50 & 8 & 9 \\
\hline$G$ & Suburban & Solidago spp. L. & 0.67 & 42 & 42 \\
\hline $\mathrm{G}$ & Suburban & Toxicodendron radicans (L.) Kuntze & 1.96 & 25 & 27 \\
\hline & Suburban & Verbesina alternifolia (L.) Britt. ex Kearney & 2.50 & 58 & 61 \\
\hline Gl & Suburban & Boehmeria cylindrica (L.) Sw. & 0.92 & 25 & 26 \\
\hline G12 & Suburban & Euonymus fortunei (Turcz.) Hand.-Maz. * & 19.17 & 50 & 69 \\
\hline
\end{tabular}




\section{Appendix Table 12 (cont.)}

\begin{tabular}{|c|c|c|c|c|c|}
\hline G12 & Suburban & Grass & 0.17 & 8 & 9 \\
\hline G12 & Suburban & Impatiens capensis Meerb. & 0.21 & 17 & 17 \\
\hline 12 & Suburban & Lonicera japonica Thunb.* & 0.96 & 58 & 59 \\
\hline 12 & Suburban & Maianthemum racemosum (L.) Link & 0.58 & 17 & 17 \\
\hline 12 & Suburban & Parthenocissus quinquefolia (L.) Planch. & 13.38 & 92 & 105 \\
\hline 2 & Suburban & $\begin{array}{l}\text { Sanicula odorata (Raf.) K.M. Pryer \& L.R. } \\
\text { Phillippe }\end{array}$ & 0.33 & 25 & 25 \\
\hline 12 & Suburban & Toxicodendron radicans (L.) Kuntze & 0.63 & 17 & 17 \\
\hline 2 & Suburban & Verbesina alternifolia (L.) Britt. ex Kearney & 5.33 & 75 & 80 \\
\hline 12 & Suburban & Viola spp. L. & 0.08 & 8 & \\
\hline 2 & Suburban & Woodsia obtusa (Spreng.) Torr. & 0.08 & 8 & 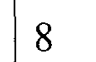 \\
\hline 13 & Suburban & $\begin{array}{l}\text { Ageratina altissima (L.) King \& H.E. } \\
\text { Robins. }\end{array}$ & 3.63 & 83 & 87 \\
\hline 13 & Suburban & Arundinaria gigantea (Walt.) Muhl. & 17.67 & 92 & 09 \\
\hline 13 & Suburban & Desmodium spp. L. & 0.29 & 25 & 5 \\
\hline 13 & Suburban & Duchesnea indica (Andr.) Focke* & 1.17 & 42 & 43 \\
\hline 13 & Suburban & Euonymus fortunei (Turcz.) Hand.-Maz. * & 0.13 & 17 & 17 \\
\hline 13 & Suburban & Glechoma hederacea L.* & 1.13 & 100 & 101 \\
\hline 3 & Suburban & Grass & 0.33 & 17 & 17 \\
\hline 13 & Suburban & Impatiens capensis Meerb. & 0.04 & 8 & $\gamma$ \\
\hline G13 & Śburban & Microstegium vimineum (Trin.) A. Camus * & 1.67 & 33 & 35 \\
\hline 13 & Suburban & Pilea pumila (L.) Gray & 0.63 & 42 & 42 \\
\hline 13 & Suburban & Symphyotrichum spp. L. & 0.13 & 17 & 17 \\
\hline 3 & Suburban & Verbesina alternifolia (L.) Britt. ex Kearney & 0.42 & 17 & 17 \\
\hline 3 & Suburban & Viola spp. L. & 0.21 & 42 & 42 \\
\hline 13 & Suburban & Viola striata Ait. & 0.21 & 17 & 17 \\
\hline 14 & Suburban & Erigeron philadelphicus $\mathrm{L}$. & 3.67 & 67 & 70 \\
\hline 14 & Suburban & Glechoma hederacea L.* & 0.67 & 33 & 34 \\
\hline 14 & Suburban & Grass & 95.00 & 100 & 195 \\
\hline 14 & Suburban & Pilea pumila (L.) Gray & 2.33 & 33 & 36 \\
\hline 14 & Suburban & Plantago rugelii Dcne. & 0.50 & 33 & 34 \\
\hline G14 & Suburban & Ruellia caroliniensis (J.F. Gmel.) Steud. & 2.00 & 67 & 69 \\
\hline 14 & Suburban & Rumex verticillatus $\mathrm{L}$. & 0.33 & 33 & 34 \\
\hline 14 & Suburban & Symphyotrichum lowrieanum (Porter) Nesom & 0.67 & 33 & 34 \\
\hline & Suburban & Viola spp. L. & 0.33 & 33 & 34 \\
\hline & Suburban & $\begin{array}{l}\text { Ageratina altissima (L.) King \& H.E. } \\
\text { Robins. }\end{array}$ & 0.54 & 42 & 12 \\
\hline & Suburban & Aristolochia tomentosa Sims & 0.17 & 8 & 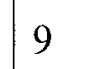 \\
\hline & Suburban & Euonymus fortunei (Turcz.) Hand.-Maz. * & 2.50 & 42 & 44 \\
\hline & Suburban & Impatiens capensis Meerb. & 0.04 & 8 & 8 \\
\hline
\end{tabular}




\section{Appendix Table 12 (cont.)}

\begin{tabular}{|c|c|c|c|c|c|}
\hline G3 & Suburban & Juncus spp. L. & 0.21 & 25 & 25 \\
\hline G3 & Suburban & Lysimachia nummularia L.* & 1.75 & 25 & 27 \\
\hline 3 & Suburban & $\begin{array}{l}\text { Sanicula odorata (Raf.) K.M. Pryer \& L.R. } \\
\text { Phillippe }\end{array}$ & 0.17 & 17 & 17 \\
\hline & Suburban & Solidago spp. L. & 2.46 & 67 & 69 \\
\hline & Suburban & Toxicodendron radicans (L.) Kuntze & 0.67 & 25 & 26 \\
\hline & Suburban & Viola spp. L. & 11.54 & 67 & 78 \\
\hline & Suburban & $\begin{array}{l}\text { Ageratina altissima (L.) King \& H.E. } \\
\text { Robins. }\end{array}$ & 2.38 & 42 & 44 \\
\hline & Suburban & Alliaria petiolata (Bieb.) Cavara \& Grande * & 0.04 & 8 & 0 \\
\hline & Suburban & Arundinaria gigantea (Walt.) Muhl. & 8.75 & 100 & 109 \\
\hline & Suburban & Asarum canadense L. & 0.04 & 8 & \\
\hline & Suburban & Cystopteris tenuis (Michx.) Desv. & 0.13 & 17 & 17 \\
\hline & Suburban & Duchesnea indica (Andr.) Focke* & 0.50 & 17 & 17 \\
\hline & Suburban & Glechoma hederacea L.* & 34.92 & 83 & 118 \\
\hline & Suburban & Grass & 0.08 & 8 & \\
\hline & Suburban & Impatiens capensis Meerb. & 0.21 & 25 & 25 \\
\hline & Suburban & Lamiaceae & 0.33 & 42 & 42 \\
\hline & Suburban & $\begin{array}{l}\text { Sanicula odorata (Raf.) K.M. Pryer \& L.R. } \\
\text { Phillippe }\end{array}$ & 0.21 & 25 & \\
\hline & Suburban & Solidago spp. L. & 0.13 & 8 & 0 \\
\hline & Suburban & Verbesina alternifolia (L.) Britt. ex Kearney & 0.58 & 17 & 17 \\
\hline & Suburban & Viola spp. L. & 0.04 & 8 & 0 \\
\hline 9 & Suburban & $\begin{array}{l}\text { Ageratina altissima (L.) King \& H.E. } \\
\text { Robins. }\end{array}$ & 4.58 & 75 & 0 \\
\hline & Suburban & Duchesnea indica (Andr.) Focke* & 0.38 & 25 & 25 \\
\hline & Suburban & Euonymus fortunei (Turcz.) Hand.-Maz. * & 0.08 & 8 & 8 \\
\hline & Suburban & Galium aparine $\mathrm{L}$. & 0.04 & 8 & 8 \\
\hline G9 & Suburban & Glechoma hederacea L.* & 41.38 & 42 & 83 \\
\hline & Suburban & Grass & 0.83 & 25 & 26 \\
\hline & Suburban & Impatiens capensis Meerb. & 2.21 & 42 & 44 \\
\hline & Suburban & Lysimachia nummularia L.* & 0.25 & 8 & 9 \\
\hline & Suburban & $\begin{array}{l}\text { Sanicula odorata (Raf.) K.M. Pryer \& L.R. } \\
\text { Phillippe }\end{array}$ & 0.83 & 75 & 6 \\
\hline & Suburban & Smilax rotundifolia $\mathrm{L}$. & 0.08 & 8 & 8 \\
\hline & Suburban & Solidago spp. L. & 7.17 & 75 & 82 \\
\hline & Suburban & Toxicodendron radicans (L.) Kuntze & 0.04 & 8 & 8 \\
\hline & Suburban & Verbesina alternifolia (L.) Britt. ex Kearney & 1.08 & 25 & 26 \\
\hline & Suburban & Viola spp. L. & 2.54 & 75 & 78 \\
\hline & Suburban & Viola striata Ait. & 2.38 & 58 & 61 \\
\hline 10 & Suburban & Ageratina altissima (L.) King \& H.E. Robins. & 1.50 & 73 & 74 \\
\hline
\end{tabular}




\section{Appendix Table 12 (cont.)}

\begin{tabular}{|c|c|c|c|c|c|}
\hline $\mathrm{H} 10$ & Suburban & Lonicera japonica Thunb.* & 2.91 & 45 & 48 \\
\hline $\mathrm{H} 10$ & Suburban & $\begin{array}{l}\text { Packera obovata (Muhl. ex Willd.) W.A. } \\
\text { Weber \& A. Löve }\end{array}$ & 0.05 & 9 & 9 \\
\hline $\mathrm{H} 10$ & Suburban & $\begin{array}{l}\text { Sanicula odorata (Raf.) K.M. Pryer \& L.R. } \\
\text { Phillippe }\end{array}$ & 5.41 & 91 & 96 \\
\hline $\mathrm{H} 10$ & Suburban & Symphyotrichum cordifolium (L.) Nesom & 0.14 & 18 & 18 \\
\hline $\mathrm{H} 2$ & Suburban & $\begin{array}{l}\text { Ageratina altissima }(\text { L.) King \& H.E. } \\
\text { Robins. }\end{array}$ & 2.00 & 67 & 69 \\
\hline $\mathrm{H} 2$ & Suburban & Asarum canadense L. & 1.42 & 67 & 68 \\
\hline $\mathrm{H} 2$ & Suburban & Apiaceae & 0.04 & 8 & 0 \\
\hline $\mathrm{H} 2$ & Suburban & Euonymus fortunei (Turcz.) Hand.-Maz. * & 0.58 & 58 & 59 \\
\hline $\mathrm{H} 2$ & Suburban & Glechoma hederacea L.* & 1.63 & 83 & 85 \\
\hline $\mathrm{H} 2$ & Suburban & Grass & 0.92 & 58 & 59 \\
\hline $\mathrm{H} 2$ & Suburban & $\begin{array}{l}\text { Sanicula odorata (Raf.) K.M. Pryer \& L.R. } \\
\text { Phillippe }\end{array}$ & 0.58 & 67 & 67 \\
\hline $\mathrm{H} 2$ & Suburban & Smilax rotundifolia $\mathrm{L}$. & 0.17 & 8 & 9 \\
\hline $\mathrm{H} 2$ & Suburban & Symphyotrichum spp. L. & 0.42 & 25 & 25 \\
\hline $\mathrm{H}$ & Suburban & $\begin{array}{l}\text { Thalictrum thalictroides (L.) Eames \& } \\
\text { Boivin }\end{array}$ & 0.04 & 8 & 8 \\
\hline $\mathrm{H}$ & Suburban & Viola spp. L. & 0.92 & 58 & 59 \\
\hline $\mathrm{H} 4$ & Suburban & $\begin{array}{l}\text { Ageratina altissima (L.) King \& H.E. } \\
\text { Robins. }\end{array}$ & 0.25 & 33 & 34 \\
\hline $\mathrm{H} 4$ & Suburban & Ampelamus albidus (Nutt.) Britt. & 0.08 & 17 & 17 \\
\hline $\mathrm{H} 4$ & Suburban & Asarum canadense $\mathrm{L}$. & 1.75 & 100 & 102 \\
\hline $\mathrm{H} 4$ & Suburban & Lactuca canadensis $\mathrm{L}$. & 0.08 & 17 & 17 \\
\hline $\mathrm{H} 4$ & Suburban & Microstegium vimineum (Trin.) A. Camus * & 0.67 & 17 & 17 \\
\hline $\mathrm{H} 4$ & Suburban & Viola spp. L. & 0.08 & 17 & 17 \\
\hline $\mathrm{H} 4$ & Suburban & Viola striata Ait. & 0.08 & 17 & 17 \\
\hline B1 & Urban & Ageratina spp. L. & 0.13 & 2 & 2 \\
\hline B1 & Urban & Alliaria petiolata (Bieb.) Cavara \& Grande * & 7.13 & 75 & 82 \\
\hline B1 & Urban & Asarum canadense $\mathrm{L}$. & 0.02 & 4 & 4 \\
\hline B1 & Urban & Duchesnea indica (Andr.) Focke* & 0.83 & 25 & 26 \\
\hline B1 & Urban & Erigeron spp. L. & 0.04 & 4 & 4 \\
\hline B1 & Urban & Euonymus fortunei (Turcz.) Hand.-Maz. * & 0.17 & 8 & 9 \\
\hline Bl & Urban & Glechoma hederacea L.* & 18.10 & 79 & 97 \\
\hline B1 & Urban & Impatiens capensis Meerb. & 3.00 & 63 & 66 \\
\hline B1 & Urban & Lamium amplexicaule $\mathrm{L}$. & 0.21 & 4 & 4 \\
\hline B1 & Urban & Lonicera japonica Thunb.* & 0.04 & 4 & 4 \\
\hline B1 & Urban & Sanicula spp. L. & 0.31 & 8 & 9 \\
\hline B1 & Urban & Sicyos angulatus $\mathrm{L}$. & 0.42 & 25 & 25 \\
\hline B1 & Urban & Solidago spp. L. & 1.37 & 42 & 43 \\
\hline
\end{tabular}




\begin{tabular}{|c|c|c|c|c|c|}
\hline & & & & & \\
\hline B1 & Urban & Urtica dioica $\mathrm{L}$. & 1.88 & 29 & 31 \\
\hline $\mathrm{B} 1$ & Urban & Verbesina alternifolia (L.) Britt. ex Kearney & 1.81 & 21 & 23 \\
\hline B1 & Urban & Viola spp. L. & 0.06 & 8 & 10 \\
\hline B13 & Urban & $\begin{array}{l}\text { Ageratina altissima (L.) King \& H.E. } \\
\text { Robins. }\end{array}$ & 1.83 & 33 & 35 \\
\hline B13 & Urban & Helianthus spp. L. & 1.17 & 33 & J \\
\hline B13 & Urban & Verbesina alternifolia (L.) Britt. ex Kearney & 0.25 & 17 & 17 \\
\hline B13 & Urban & Vitis vulpina $\mathrm{L}$. & 0.08 & 17 & 17 \\
\hline $\mathrm{B} 14$ & Urban & Alliaria petiolata (Bieb.) Cavara \& Grande * & 0.17 & 17 & 17 \\
\hline B14 & Urban & Asarum canadense L. & 1.25 & 17 & 18 \\
\hline B14 & Urban & Cystopteris tenuis (Michx.) Desv. & 0.08 & 8 & 8 \\
\hline B14 & Urban & Duchesnea indica (Andr.) Focke* & 0.08 & 8 & 8 \\
\hline B14 & Urban & Euonymus fortunei (Turcz.) Hand.-Maz. * & 53.08 & 100 & 153 \\
\hline B14 & Urban & Smilax rotundifolia $\mathrm{L}$. & 0.04 & 8 & 8 \\
\hline B14 & Urban & Urtica dioica $\mathrm{L}$. & 1.92 & 42 & 44 \\
\hline $\mathrm{B} 2$ & Urban & Ageratina spp. L. & 1.46 & 50 & 51 \\
\hline B2 & Urban & Asarum canadense L. & 1.83 & 25 & 27 \\
\hline B2 & Urban & Apiaceae & 0.17 & 8 & 17 \\
\hline B2 & Urban & Duchesnea indica (Andr.) Focke* & 0.04 & 8 & 8 \\
\hline B2 & Urban & Euonymus fortunei (Turcz.) Hand.-Maz. * & 27.00 & 83 & 110 \\
\hline B2 & Urban & Glechoma hederacea L.* & 2.29 & 50 & 52 \\
\hline B2 & Urban & Grass & 11.50 & 67 & 78 \\
\hline B2 & Urban & Microstegium vimineum (Trin.) A. Camus * & 0.17 & 8 & 9 \\
\hline B2 & Urban & $\begin{array}{l}\text { Packera obovata (Muhl. ex Willd.) W.A. } \\
\text { Weber \& A. Löve }\end{array}$ & 0.04 & 8 & 8 \\
\hline B2 & Urban & $\begin{array}{l}\text { Sanicula odorata (Raf.) K.M. Pryer \& L.R. } \\
\text { Phillippe }\end{array}$ & 0.92 & 42 & 43 \\
\hline B2 & Urban & Smilax rotundifolia $\mathrm{L}$. & 1.08 & 8 & 9 \\
\hline B2 & Urban & Toxicodendron radicans (L.) Kuntze & 2.21 & 50 & 52 \\
\hline B2 & Urban & Urtica dioica $\mathrm{L}$. & 1.92 & 8 & 10 \\
\hline B2 & Urban & Verbesina alternifolia (L.) Britt. ex Kearney & 0.54 & 25 & 26 \\
\hline B2 & Urban & Viola spp. L. & 0.63 & 33 & 34 \\
\hline B2 & Urban & Vitis vulpina $\mathrm{L}$. & 0.17 & 17 & 17 \\
\hline B3 & Urban & Euonymus fortunei (Turcz.) Hand.-Maz. * & 14.67 & 50 & 65 \\
\hline B3 & Urban & Parthenocissus quinquefolia (L.) Planch. & 0.04 & 8 & 8 \\
\hline B3 & Urban & Polygonatum biflorum (Walt.) Ell. & 0.75 & 17 & 17 \\
\hline B3 & Urban & Smilax rotundifolia $\mathrm{L}$. & 0.04 & 8 & 8 \\
\hline B3 & Urban & Toxicodendron radicans (L.) Kuntze & 0.04 & 8 & 8 \\
\hline B4 & Urban & Ageratina spp. L. & 0.13 & 8 & 8 \\
\hline B4 & Urban & Alliaria petiolata (Bieb.) Cavara \& Grande * & 9.67 & 50 & 60 \\
\hline
\end{tabular}




\begin{tabular}{|c|c|c|c|c|c|}
\hline & & Appendix Table 12 (cont.) & & & \\
\hline B4 & Urban & Cardamine pensylvanica Muhl. ex Willd. & 0.04 & 8 & 8 \\
\hline B4 & Urban & Euonymus fortunei (Turcz.) Hand.-Maz. * & 68.08 & 100 & 168 \\
\hline B4 & Urban & Impatiens capensis Meerb. & 0.42 & 25 & 25 \\
\hline B5 & Urban & $\begin{array}{l}\text { Ageratina altissima (L.) King \& H.E. } \\
\text { Robins. }\end{array}$ & 7.00 & 42 & 49 \\
\hline $\mathrm{B}$ & Urban & Euonymus fortunei (Turcz.) Hand.-Maz. * & 36.33 & 67 & 103 \\
\hline 5 & Urban & Toxicodendron radicans (L.) Kuntze & 0.08 & 8 & 8 \\
\hline B5 & Urban & Verbesina alternifolia (L.) Britt. ex Kearney & 2.21 & 25 & 27 \\
\hline B6 & Urban & $\begin{array}{l}\text { Ageratina altissima (L.) King \& H.E. } \\
\text { Robins. }\end{array}$ & 17.25 & 83 & 101 \\
\hline 0 & Urban & Ampelamus albidus (Nutt.) Britt. & 0.58 & 33 & 34 \\
\hline B6 & Urban & Duchesnea indica (Andr.) Focke* & 0.42 & 33 & 34 \\
\hline B6 & Urban & Solidago spp. L. & 3.92 & 83 & 87 \\
\hline B6 & Urban & Verbesina alternifolia (L.) Britt. ex Kearney & 0.50 & 17 & 17 \\
\hline B6 & Urban & Viola spp. L. & 1.00 & 33 & 34 \\
\hline B7 & Urban & $\begin{array}{l}\text { Ageratina altissima (L.) King \& H.E. } \\
\text { Robins. }\end{array}$ & 10.57 & 63 & 73 \\
\hline B7 & Urban & Alliaria petiolata (Bieb.) Cavara \& Grande * & 0.27 & 8 & 9 \\
\hline B7 & Urban & Aureolaria spp. L. & 0.02 & 4 & 4 \\
\hline B7 & Urban & Cystopteris tenuis (Michx.) Desv. & 0.04 & 8 & 8 \\
\hline B7 & Urban & Duchesnea indica (Andr.) Focke* & 0.33 & 17 & 17 \\
\hline B7 & Urban & Elymus riparius Wieg. & 0.17 & 4 & 4 \\
\hline B7 & Urban & Euonymus fortunei (Turcz.) Hand.-Maz. * & 0.71 & 13 & 13 \\
\hline B7 & Urban & Glechoma hederacea L.* & 1.90 & 25 & 27 \\
\hline B7 & Urban & Grass & 6.92 & 54 & 61 \\
\hline B7 & Urban & Lonicera japonica Thunb.* & 0.29 & 13 & 13 \\
\hline B7 & Urban & Lysimachia nummularia L.* & 0.13 & 4 & 4 \\
\hline B7 & Urban & Oxalis stricta L. & 0.02 & 4 & 4 \\
\hline B7 & Urban & Plantago rugelii Dcne. & 0.11 & 13 & 13 \\
\hline B7 & Urban & Ranunculus hispidus Michx. & 0.02 & 4 & 4 \\
\hline B7 & Urban & Sanicula spp. L. & 0.25 & 4 & 4 \\
\hline B7 & Urban & $\begin{array}{l}\text { Sanicula odorata (Raf.) K.M. Pryer \& L.R. } \\
\text { Phillippe }\end{array}$ & 1.24 & 38 & 39 \\
\hline B & Urban & Smilax rotundifolia $\mathrm{L}$. & 0.15 & 8 & 8 \\
\hline B7 & Urban & Solidago spp. L. & 1.31 & 25 & 26 \\
\hline B7 & Urban & Toxicodendron radicans (L.) Kuntze & 0.12 & 8 & 8 \\
\hline B7 & Urban & Verbesina alternifolia (L.) Britt. ex Kearney & 13.32 & 84 & 97 \\
\hline B7 & Urban & Viola spp. L. & 0.32 & 17 & 17 \\
\hline B8 & Urban & Aristolochia tomentosa Sims & 0.13 & 8 & 8 \\
\hline B8 & Urban & Euonymus fortunei (Turcz.) Hand.-Maz. * & 0.42 & 25 & 25 \\
\hline B8 & Urban & Juncus spp. L. & 0.21 & 8 & 9 \\
\hline
\end{tabular}




\begin{tabular}{|c|c|c|c|c|c|}
\hline \multicolumn{6}{|c|}{ Appendix Table 12 (cont.) } \\
\hline B8 & Urban & Oxalis stricta $\mathrm{L}$. & 0.17 & 17 & 17 \\
\hline B8 & Urban & Phytolacca americana $\mathrm{L}$. & 0.17 & 8 & 9 \\
\hline B8 & Urban & Solidago spp. L. & 2.21 & 17 & 19 \\
\hline B8 & Urban & Viola spp. L. & 0.08 & 8 & 8 \\
\hline B9 & Urban & Sorghum halepense (L.) Pers.* & 0.08 & 8 & 8 \\
\hline B9 & Urban & Toxicodendron radicans (L.) Kuntze & 0.13 & 8 & 8 \\
\hline G8 & Urban & $\begin{array}{l}\text { Ageratina altissima (L.) King \& H.E. } \\
\text { Robins. }\end{array}$ & 3.13 & 50 & 53 \\
\hline G8 & Urban & Carex blanda Dewey & 12.56 & 25 & 38 \\
\hline G8 & Urban & Euonymus fortunei (Turcz.) Hand.-Maz. * & 0.06 & 13 & 13 \\
\hline G8 & Urban & Glechoma hederacea L.* & 3.19 & 63 & 66 \\
\hline G8 & Urban & $\begin{array}{l}\text { Sanicula odorata (Raf.) K.M. Pryer \& L.R. } \\
\text { Phillippe }\end{array}$ & 0.13 & 25 & 25 \\
\hline G8 & Urban & Verbesina alternifolia (L.) Britt. ex Kearney & 3.00 & 25 & 28 \\
\hline G8 & Urban & Viola spp. L. & 0.06 & 13 & 13 \\
\hline
\end{tabular}

Non-native species indicated with an $\left({ }^{*}\right)$.

Note: Plants were identified to the most distinct taxonomic group possible.

Land use categories based upon $\%$ impervious surface at the subcatchment scale. 
Appendix Table 13: Soil properties and trace nutrients from soil samples removed from static chamber centers located in riparian sites in urban, suburban, and rural subcatchments in October 2008. Urban subcatchments contain $\geq 30 \%$ impervious surface cover, rural subcatchments contain $\leq 10 \%$ impervious surface cover, and suburban subcatchments contain values in between 10 and $30 \%$ impervious surface cover. Site names beginning with $\mathrm{R}, \mathrm{S}$, and $\mathrm{U}$ are located in rural, suburban, and urban subcatchments, respectively. The second letter in the site name indicates whether the site is located in Harrods $(\mathrm{H})$, Goose $(\mathrm{G})$, or Beargrass $(\mathrm{B})$ creek watershed.

\begin{tabular}{|c|c|c|c|c|c|c|c|c|}
\hline Site & $\begin{array}{c}\text { Sand } \\
(\%) \\
\end{array}$ & $\begin{array}{l}\text { Silt } \\
(\%) \\
\end{array}$ & $\begin{array}{l}\text { Clay } \\
(\%) \\
\end{array}$ & $\begin{array}{c}\text { TEC } \\
\text { (meq/ } \\
100 \mathrm{~g}) \\
\end{array}$ & $\begin{array}{c}\text { pH (1:1 } \\
\text { soil to } \\
\text { water }) \\
\end{array}$ & $\begin{array}{c}\text { Organic } \\
\text { Matter } \\
(\%) \\
\end{array}$ & $\begin{array}{c}\mathrm{S} \\
(\mathrm{mg} / \\
\mathrm{kg})\end{array}$ & $\begin{array}{r}\text { Bulk } \\
\text { dens. }\end{array}$ \\
\hline RG4 & 19.9 & 65.1 & 15.0 & 12.9 & 7.1 & 4.48 & 21 & 0.59 \\
\hline RG7 & 17.7 & 67.2 & 15.1 & 13.8 & 7.1 & 4.06 & 36 & 0.53 \\
\hline RH13 & 27.3 & 54.8 & 18.0 & 21.8 & 6.6 & 9.98 & 44 & 0.73 \\
\hline RH5 & 43.2 & 43.2 & 13.6 & 21.1 & 7.2 & 3.4 & 29 & 0.52 \\
\hline SB12 & 7.8 & 80.8 & 11.5 & 22.3 & 7.6 & 5.06 & 38 & 0.56 \\
\hline SG13 & 21.1 & 64.1 & 14.9 & 14.4 & 7.3 & 4.95 & 36 & 0.58 \\
\hline SG9 & 10.9 & 72.5 & 16.6 & 13.3 & 7.5 & 3.5 & 23 & 0.56 \\
\hline UB1 & 11.3 & 67.9 & 20.8 & 17.1 & 7.2 & 4.74 & 36 & 0.63 \\
\hline UB14 & 22.6 & 67.5 & 10.0 & 28.4 & 6.9 & 6.49 & 81 & 0.49 \\
\hline UB2 & 28.9 & 54.9 & 16.2 & 20.0 & 7.7 & 3.98 & 26 & 0.66 \\
\hline UB3 & 15.8 & 66.3 & 17.9 & 15.5 & 7.0 & 4.78 & 37 & 0.5 \\
\hline UB5 & 8.3 & 70.6 & 21.1 & 13.1 & 7.4 & 6.36 & 30 & 0.55 \\
\hline UG8 & 28.3 & 59.1 & 12.6 & 11.6 & 6.1 & 4.15 & 31 & 0.58 \\
\hline
\end{tabular}

Brookside Laboratories, 308 S. Main Street, New Knoxville, OH, 45871, 419-7532448, www.blinc.com 
Appendix Table 13 (cont.)

\begin{tabular}{|c|c|c|c|c|c|c|c|c|c|c|}
\hline Site & $\begin{array}{c}\text { Bray II } \\
\text { P } \\
(\mathrm{mg} / \mathrm{kg})\end{array}$ & $\begin{array}{c}\mathrm{Ca} \\
(\mathrm{mg} / \\
\mathrm{kg})\end{array}$ & $\begin{array}{c}\mathrm{Mg} \\
(\mathrm{mg} / \\
\mathrm{kg})\end{array}$ & $\begin{array}{c}\mathrm{K} \\
(\mathrm{mg} / \\
\mathrm{kg})\end{array}$ & $\begin{array}{c}\mathrm{Na} \\
(\mathrm{mg} / \\
\mathrm{kg})\end{array}$ & $\begin{array}{c}\mathrm{Ca} \\
(\%)\end{array}$ & $\begin{array}{l}\mathrm{Mg} \\
(\%)\end{array}$ & $\begin{array}{c}K \\
(\%)\end{array}$ & $\begin{array}{c}\mathrm{Na} \\
(\%)\end{array}$ & $\begin{array}{c}\mathbf{H} \\
(\%)\end{array}$ \\
\hline RG4 & 113 & 1992 & 251 & 81 & 22 & 77.2 & 16.2 & 1.61 & 0.74 & 0 \\
\hline RG7 & 75 & 2143 & 269 & 51 & 26 & 77.7 & 16.3 & 0.95 & 0.82 & 0 \\
\hline RH13 & 158 & 3410 & 232 & 128 & 22 & 78.4 & 8.9 & 1.51 & 0.44 & 6 \\
\hline RH5 & 114 & 3694 & 168 & 54 & 40 & 87.7 & 6.6 & 0.66 & 0.83 & 0 \\
\hline SB12 & 55 & 3843 & 238 & 66 & 23 & 86.1 & 8.9 & 0.76 & 0.45 & 0 \\
\hline SG13 & 63 & 2002 & 411 & 94 & 22 & 69.7 & 23.9 & 1.68 & 0.67 & 0 \\
\hline SG9 & 121 & 2033 & 271 & 75 & 32 & 76.6 & 17.0 & 1.45 & 1.05 & 0 \\
\hline UB1 & 151 & 2559 & 404 & 58 & 26 & 74.7 & 19.6 & 0.87 & 0.66 & 0 \\
\hline UB14 & 122 & 4787 & 283 & 102 & 27 & 84.4 & 8.3 & 0.92 & 0.41 & 1.5 \\
\hline UB2 & 11 & 3395 & 237 & 69 & 22 & 85.1 & 9.9 & 0.89 & 0.48 & 0 \\
\hline UB3 & 86 & 2568 & 208 & 61 & 22 & 82.8 & 11.2 & 1.01 & 0.62 & 0 \\
\hline UB5 & 53 & 1901 & 340 & 79 & 18 & 72.3 & 21.6 & 1.54 & 0.60 & 0 \\
\hline UG8 & 122 & 1428 & 213 & 157 & 22 & 61.7 & 15.3 & 3.48 & 0.83 & 14 \\
\hline
\end{tabular}


Appendix Table 13 (cont.)

\begin{tabular}{lccccccc} 
Site & $\begin{array}{c}\text { B } \\
(\mathbf{m g} / \mathbf{k g})\end{array}$ & $\begin{array}{c}\text { Fe } \\
(\mathbf{m g} / \mathbf{k g})\end{array}$ & $\begin{array}{c}\text { Mn } \\
(\mathbf{m g} / \mathbf{k g})\end{array}$ & $\begin{array}{c}\mathbf{C u} \\
(\mathbf{m g} / \mathbf{k g})\end{array}$ & $\begin{array}{c}\mathbf{Z n} \\
(\mathbf{m g} / \mathbf{k g})\end{array}$ & $\begin{array}{c}\text { Al } \\
(\mathbf{m g} / \mathbf{k g})\end{array}$ & $\begin{array}{c}\text { P } \\
(\mathbf{m g} / \mathbf{k g})\end{array}$ \\
\hline RG4 & 0.97 & 186 & 317 & 5.16 & 8.74 & 461 & 26 \\
\hline RG7 & 0.85 & 226 & 472 & 4.45 & 11.42 & 403 & 45 \\
\hline RH13 & 1.09 & 324 & 284 & 4.78 & 11.47 & 404 & 97 \\
\hline RH5 & 0.60 & 208 & 334 & 6.83 & 15.22 & 371 & 61 \\
\hline SB12 & 0.98 & 218 & 411 & 3.59 & 5.99 & 290 & 41 \\
\hline SG13 & 0.93 & 152 & 409 & 3.97 & 11.26 & 439 & 24 \\
\hline SG9 & 0.77 & 187 & 419 & 6.01 & 12.87 & 478 & 41 \\
\hline UB1 & 0.86 & 289 & 464 & 7.67 & 16.59 & 467 & 92 \\
\hline UB14 & 0.99 & 398 & 476 & 5.15 & 9.69 & 339 & 97 \\
\hline UB2 & 0.70 & 173 & 356 & 2.04 & 3.27 & 254 & 13 \\
\hline UB3 & 0.64 & 195 & 361 & 4.89 & 12.98 & 363 & 34 \\
\hline UB5 & 0.96 & 153 & 525 & 2.02 & 4.57 & 359 & 22 \\
\hline UG8 & 0.64 & 262 & 311 & 5.52 & 19.55 & 608 & 102 \\
\hline
\end{tabular}

Methods for the data collected are referenced as follows: Total exchange capacity (Ross 1995); pH (McLean 1982); organic matter (Schulte and Hopkins 1996); S, Ca, Mg, K, $\mathrm{Na}, \mathrm{B}, \mathrm{Fe}, \mathrm{Mn}, \mathrm{Cu}, \mathrm{Zn}, \mathrm{Al}$, and P (Mehlich 1984); soil texture (ASTM Standard D422-63 2002); Bray-II Phosphorus (Bray and Kurtz 1945); Zn, Mn, Fe, and Cu (Linsay and Norvell 1978). 
Appendix Table 14: Monthly mean carbon dioxide flux rates $\left(\mathrm{mg} \mathrm{CO}_{2}-\mathrm{C} / \mathrm{m}^{2} * \mathrm{~d}\right)$ from riparian soils within urban, suburban, and rural subcatchments from January to October 2008.

\begin{tabular}{l|c|c|c|c|c|c|c|c|c|c} 
Site & Jan & Feb & March & April & May & June & July & Aug & Sept & Oct \\
\hline RG4 & 0 & 454 & 422 & 1817 & 2377 & 3666 & 3056 & 2555 & 1927 & 1103 \\
RG7 & 994 & 276 & 1233 & 2940 & 1460 & 1512 & 1544 & 1950 & 1146 & 773 \\
RH13 & 174 & -47 & 245 & 2581 & 2377 & 2084 & 4816 & 1711 & 1699 & 1559 \\
RH5 & -255 & -359 & 225 & 1485 & 1127 & 1029 & 1836 & 1056 & 1174 & 735 \\
SB12 & - & 741 & 190 & 1794 & 2262 & 3087 & 2838 & 1260 & 2493 & 1260 \\
SG13 & 511 & 715 & 104 & 1536 & 1007 & 1387 & 1953 & 2140 & 2388 & 1366 \\
SG9 & 513 & 488 & 793 & 2189 & 1895 & 1666 & 3250 & 1593 & 1782 & 1160 \\
UB1 & 181 & -78 & 241 & 2171 & 1253 & 1642 & 2806 & 687 & 988 & 874 \\
UB14 & - & - & - & - & - & - & 5166 & 3527 & 4336 & 2840 \\
UB2 & 73 & 301 & 690 & 2800 & 3376 & 3970 & 3761 & 2591 & 2959 & 2775 \\
UB3 & 254 & 180 & 586 & 1750 & 1635 & 2524 & 2596 & 2920 & 2355 & 3532 \\
UB5 & 257 & 466 & 616 & 2116 & 1997 & 3778 & 3148 & 2612 & 3025 & 1561 \\
UG8 & 488 & 323 & 446 & 2567 & 2015 & 2665 & 4743 & 2605 & 2326 & 1523
\end{tabular}


Appendix Table 15: Monthly mean methane flux rates $\left(\mathrm{mg} \mathrm{CH}_{4}-\mathrm{C} / \mathrm{m}^{2} * \mathrm{~d}\right)$ from riparian soils within urban, suburban, and rural subcatchments from January to October 2008.

\begin{tabular}{l|c|c|c|c|c|c|c|c|c|c} 
Site & Jan & Feb & March & April & May & June & July & Aug & Sept & Oct \\
\hline RG4 & -0.10 & -0.26 & -0.15 & -0.41 & -0.26 & -0.24 & -0.29 & -0.47 & -0.55 & -0.59 \\
RG7 & 0.25 & 1.16 & 1.92 & 6.61 & 0.72 & 0.64 & 1.11 & 0.06 & 0.13 & -0.29 \\
RH13 & -0.13 & - & -0.11 & 0.01 & 0.09 & 0.39 & -0.09 & -0.31 & -0.19 & -0.16 \\
RH5 & -0.48 & -0.28 & -0.30 & -0.62 & -0.48 & 0.04 & -0.33 & -0.98 & -1.24 & -0.94 \\
SB12 & 0.03 & -0.19 & 0.08 & -0.33 & -0.44 & -0.30 & -0.82 & -1.37 & -1.30 & -1.02 \\
SG13 & -0.15 & -0.01 & -0.04 & -0.10 & -0.29 & -0.12 & 0.13 & 0.05 & -0.25 & -0.29 \\
SG9 & -0.03 & -0.22 & 0.30 & -0.25 & -0.50 & -0.43 & -1.69 & -0.79 & -1.03 & -1.14 \\
UB1 & -0.11 & 0.12 & -0.16 & 1.91 & -0.17 & -0.24 & -0.46 & -1.06 & -0.70 & -1.00 \\
UB14 & - & - & - & - & - & - & -0.49 & -0.69 & -0.62 & -0.66 \\
UB2 & 0.64 & -0.18 & -0.24 & -0.35 & -0.51 & -0.54 & -0.66 & -0.65 & -0.91 & -0.73 \\
UB3 & -0.35 & -0.21 & -0.23 & -0.51 & 0.01 & -0.11 & -0.32 & -0.88 & -0.82 & -0.79 \\
UB5 & -0.72 & -0.34 & -0.23 & -0.51 & -0.46 & -0.75 & -0.62 & -1.06 & -0.88 & -0.88 \\
UG8 & -0.41 & 0.04 & 0.01 & -0.28 & -0.65 & -0.16 & 0.07 & -0.54 & -0.97 & -0.62
\end{tabular}


Appendix Table 16: Monthly mean nitrous oxide flux rates $\left(\mathrm{mg} \mathrm{N}_{2} \mathrm{O}-\mathrm{N} / \mathrm{m}^{2} * \mathrm{~d}\right)$ from riparian soils within urban, suburban, and rural subcatchments in August and October 2008.

\begin{tabular}{l|c|c} 
Site & Aug & Oct \\
\hline RG4 & 0.15 & 0.20 \\
RG7 & 0.08 & 0.04 \\
RH13 & 0.34 & 0.05 \\
RH5 & 0.09 & 0.09 \\
SB12 & 0.05 & 0.13 \\
SG13 & 0.30 & 0.13 \\
SG9 & 0.17 & 0.10 \\
UB1 & 0.11 & 0.14 \\
UB14 & 0.23 & 0.23 \\
UB2 & 0.16 & 0.22 \\
UB3 & 0.14 & -0.02 \\
UB5 & 0.17 & 0.14 \\
UG8 & 0.17 & 0.13
\end{tabular}




\section{CURRICULUM VITAE}

\section{Education:}

2003-present PhD. Candidate, Biology, University of Louisville, Louisville, KY

Dissertation Title: Riparian corridor vegetation structure and soil function along urban, suburban, and rural streams in Louisville, KY, USA.

Advisor: Dr. Margaret Carreiro

\section{GPA: 3.67}

1999-2001 Master of Science, Biology, Western Carolina University, Cullowhee, NC Thesis: Role of the alternatively spliced Arabidopsis thaliana AtB'g5' untranslated region on translation during heat shock and development.

Advisor: Dr. Sabine Rundle

GPA: 3.84

1998-1999 Post-baccalaureate, Biology, University of North Carolina

GPA: 3.76

1991-1996 Bachelor of Science, Psychology, Western Carolina University

GPA: 3.06

\section{Research Experience:}

2004-2008 Dissertation research, University of Louisville

Studied nitrate removal and methane uptake functions from riparian zones as a means to protect our urban waterways. Determined herbaceous and woody plant community structure along streambanks in urban environments.

2007 Research Assistant, University of Louisville Conducted field and lab work studying leaf decomposition in a channelized stream and riparian area.

2001-2002 Research Assistant I, UT Southwestern Medical Center Used analytical techniques such as amphotropic transfections of mammalian T cells, radioactive isotope analysis, DNA amplification, cloning, and analysis of plasmid DNA.

2000-2001 Research Assistant, Western Carolina University Studied translation control mechanisms within Arabidopsis thaliana using techniques for RNA amplification and RNA and protein quantification. 


\section{Teaching Experience:}

2003-2009 University of Louisville, Department of Biology, Teaching Assistant Human Anatomy and Physiology Lab, 8 semesters

General Biology Lab, 1 semester

Biology for Non-majors lab, 2 semesters

General Ecology lab, 1 semester

2004 University of Louisville, Department of Minority Affairs, Adjunct

Instructor. Biology for Pre-med students, Summer term

2003 Southwestern Community College, Biology Department, Adjunct

Instructor. Human Anatomy and Physiology, Summer term

2001 Western Carolina University, Department of Biology, Teaching Assistant Human Anatomy and Physiology Lab, 1 semester

Biology for Non-majors lab, 1 semester

\section{Grants:}

2008

Kentucky Society of Natural History Research Grant (\$400)

University of Louisville Student Government Association Travel Grant (\$200)

2005 Woody Boebinger Memorial Scholarship, Kentucky Society of Natural

History $(\$ 800)$

Center for Environmental Science Matching Funds, University of

Louisville $(\$ 500)$

\section{Publications and Posters:}

2010

Voices of the next generation: Perspectives from the participants in the 2010 conservation policy Park Break session. Heath Garris, Jessica Goodrich, Annamarie Leon Guerrero, Matthew J. Heard, Meghan Lindsey, Archi Rastogi, Rodney J. White. http:/www.georgewright.org/perspectives

2008 Characterization of riparian plant communites along an urban-to-rural gradient in Louisville, KY. White, R. Jonathan and Carreiro, Margaret M. Ecological Society of America Annual Meeting. Variation in near-stream groundwater depth in urban and rural subcatchments, White, R. J. and Carreiro, M. M. Kentucky Academy of Science Annual Meeting.

2002 Functional analysis of the B' subunit of Arabidopsis protein phosphatase 2A. White, R. J., Davis, M., Esmon, C. A., Myrick, T. L., Vallee, N., Cochran, D. S., Stewart, C. N. Jr., and Rundle, S. J. Plant Science, 162: 201-209.

\section{Professional Affiliations and Meetings:}

George Wright Society

- 2010-2011, Student member

- 2010, Park Break Fellow 
Ecological Society of America

- 2006-2008, webmaster, Student Section

- 2005-2008, Student member

Kentucky Native Plant Society

- 2007-2009, interpretive native plant hike

Kentucky Society of Natural History

- 2008 , Student member

Golden Key International Honour Society, University of Louisville

- 2006 inductee

Pi Gamma Mu International Honour Society, University of North Carolina

- 1996 inductee

\section{Ad-hoc reviewer}

Journal of Environmental Quality

Urban Ecosystems

Statistical/Analytical Packages

R programming language, SAS (2 graduate level courses), PC-Ord, EstimateS

Graduate Coursework (grade in parentheses):

Conservation Biology $\left(\mathrm{B}^{+}\right)$

Evolution (B)

Biostatistics (A)

Ecosystem Ecology (A-)

Special Topics: Ecology of Urban and Suburban Landscapes (A-)

Aquatic Ecology (A-)

Population and Community Ecology (A)

Geographic Information Systems (A) 